\title{
DELINEAMENTOS ORTOGONAIS E PARCIALMENTE ORTOGONAIS: TEORIA E APLICAÇÃO
}

\author{
DENISE GARCIA DE SANTANA
}

Engenheiro Agrônomo

Orientador: Prof. Dr. ANTONIO FRANCISCO IEMMA

Tese apresentada à Escola Superior de Agricultura "Luiz de Queiroz", Universidade de São Paulo, para obtenção do título de Doutor em Agronomia. Área de Concentração: Estatística e Experimentação Agronômica.

PIRACICABA

Estado de São Paulo - Brasil

Dezembro - 2000 
Dados Internacionais de Catalogação na Publicação (CIP)

DIVISĀO DE BIBLIOTECA E DOCUMENTAÇĀO - Campus "Luiz de Queiroz"/USP

Santana, Denise Garcia de

Delineamentos ortogonais e parcialmente ortogonais: teoria e aplicação / Denise Garcia

de Santana. - - Piracicaba, 2000.

$135 \mathrm{p}$.

Tese (doutorado) - Escola Superior de Agricultura Luiz de Queiroz, 2000.

Bibliografia.

1. Análise de variância 2. Delineamento de experimento 3. Estatistica experimental 4. Modelo matemático I. Título

CDD 519.5

Permitida a copia total ou parcial deste documento, desde que citada a fonte-6. 0 -nutor: 
Aos meus pais,

\section{Martha de Moraes e Altino Santana}

DEDICO

Ao meu marido,

Carlos Alberto e a

meu filho Pedro Santana de Oliveira

OFEREÇO

À minha irmã de coração

Greicilene Pedro

AGRADEÇO 


\section{AGRADECIMENTOS}

Ao prof. Dr. Antonio Francisco Iemma, por ter ido além da função de orientador.

À CAPES/PICDT pela bolsa de estudo concedida para a realização do curso.

Ao Instituto de Ciências Agrárias da Universidade Federal de Uberlândia-MG pela oportunidade e pela espera.

Aos professores e funcionários do Departamentos de Ciências Exatas da Escola Superior de Agricultura "Luiz de Queiroz".

Agradeço a oportunidade de ter encontrado nesse longo caminho Leda Maria Gurgel do Amaral Garrido, referência de dedicação, empenho e amizade.

À minha amiga Silvia Maria de Freitas por ter me estendido a mão nos momentos mais difíceis.

Às amigas Lara Hoffmann Xavier e Jeanete Alves Moreira pelo carinho e companheirismo.

À minha secretária Sandra Maria Aparecida Matias Orsalino pela dedicação ao meu filho Pedro. 


\section{SUMARIO}

Página

RESUMO .................................................................... vii

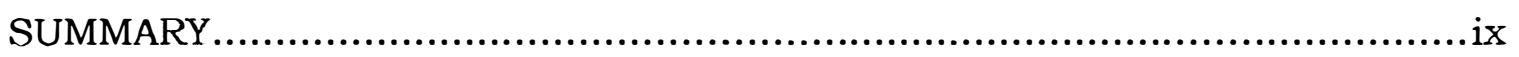

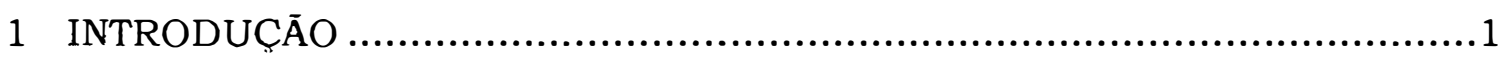

2 REVISĀO DE LITERATURA ...................................................4

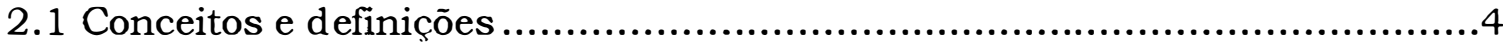

2.2 Funções estimáveis e hipóteses testáveis ...................................

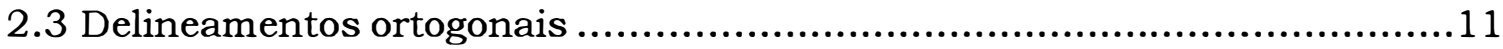

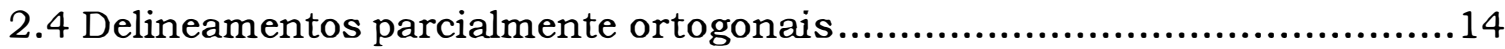

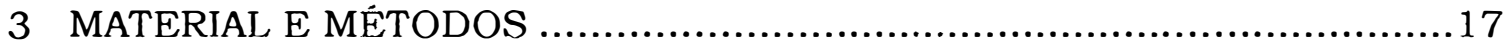

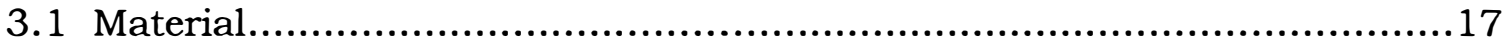

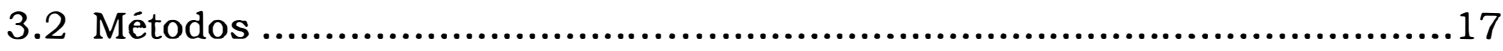

3.2.1 O termo ortogonal na análise de variância.................................18

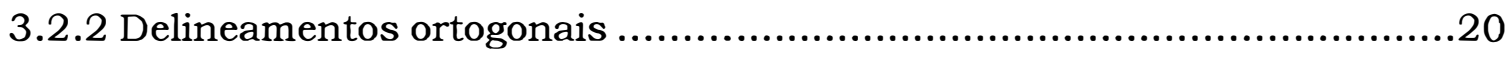

3.2.2.1 Modelos com dois fatores................................................20

3.2.2.2 Modelos com três fatores.................................................24

3.2.2.3 Modelos com quatro fatores.................................................

3.2.2.4 Modelos com $K$ fatores..................................................... 32

3.2.2.5 Propriedades dos delineamentos ortogonais ............................33

3.2.2.5.1 Quanto às funções estimáveis..........................................33

3.2.2.5.2 Quanto às hipóteses...............................................34

3.2.3 Delineamentos parcialmente ortogonais................................37

3.2.3.1 Modelos com três fatores..............................................37

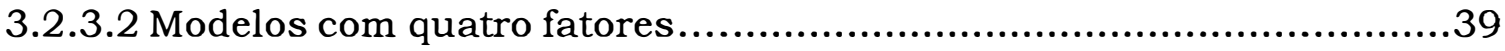

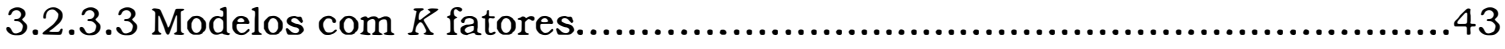

3.2.3.4 Propriedades dos delineamentos parcialmente ortogonais ...............43

3.2.3.4.1 Quanto às funções estimáveis.........................................43

3.2.3.4.2 Quanto às hipóteses...............................................44 


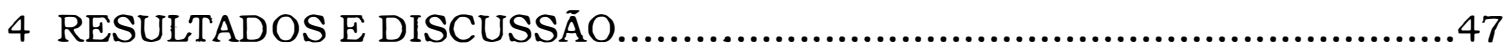

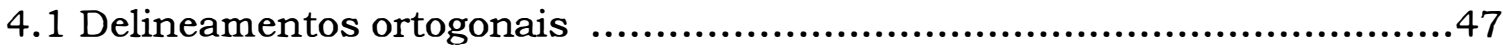

4.1.1. Modelos com dois fatores ..........................................................47

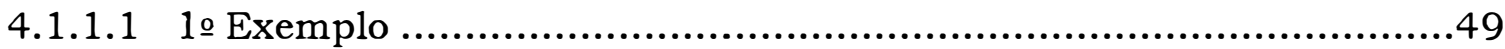

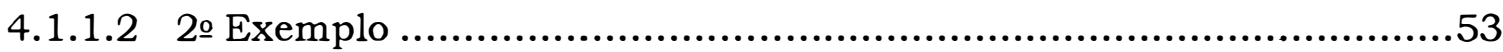

4.1.2 Modelos com três fatores...........................................................58

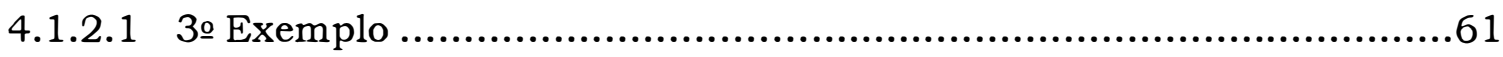

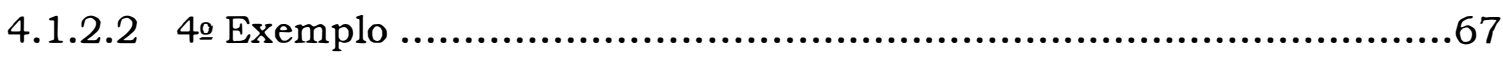

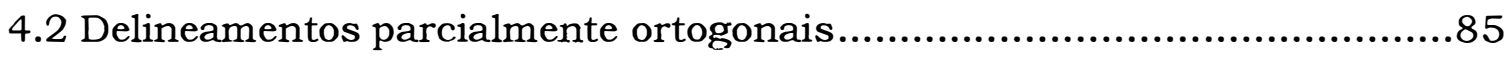

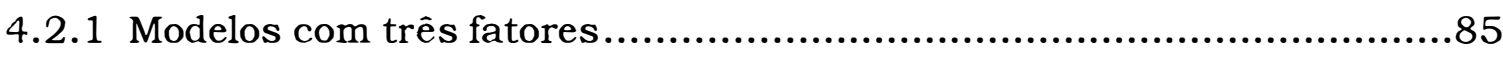

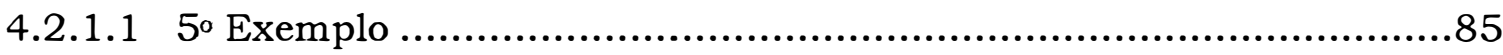

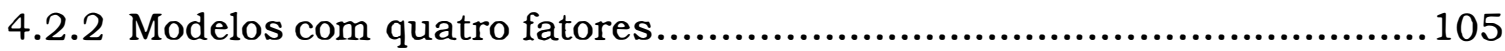

4.2.2.1 6o Exemplo .........................................................................105

4.3 Planejamento de delineamentos ortogonais e parcialmente ortogonais.. 114

4.3.1 Planejamento de delineamentos ortogonais ......................................114

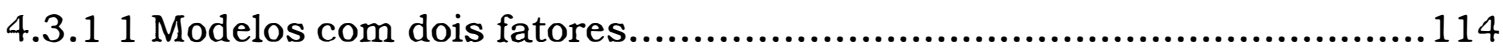

4.3.1.2 Modelos com três fatores .......................................................115

4.3.1.3 Modelos com quatro fatores...................................................116

4.3.1.4 Modelos com cinco fatores.......................................................117

4.3.2 Planejamento de delineamentos parcialmente ortogonais...................118

4.3.2.1 Modelos com três fatores.........................................................119

4.3.2.2 Modelos com quatro fatores......................................................1 120

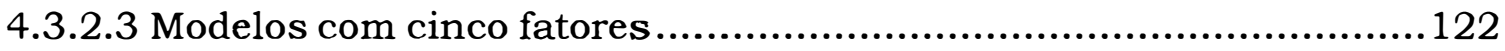

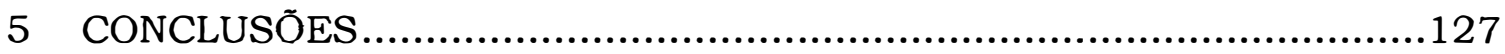

5.1 Quanto aos delineamentos ortogonais..............................................127

5.2 Quanto aos delineamentos parcialmente ortogonais ..........................129

5.3 Consideraçōes gerais......................................................................130

REFERÊNCIAS BIBLIOGRÁFICAS ................................................... 131 


\title{
DELINEAMENTOS ORTOGONAIS E PARCIALMENTE ORTOGONAIS : TEORIA E APLICAÇÃO
}

\author{
Autora: DENISE GARCIA DE SANTANA \\ Orientador: Prof. ANTONIO FRANCISCO IEMMA
}

\section{RESUMO}

Em situações experimentais, não raro, o pesquisador depara-se com a impossibilidade de planejar experimentos balanceados. Um grave problema surge imediatamente, no tocante à interpretação das hipóteses testadas através dos sistemas estatísticos, principalmente quando há vários fatores envolvidos e se faz presente um alto grau de desbalanceamento pois, em geral, as hipóteses sobre os efeitos principais de um dos fatores contêm os efeitos principais de outros fatores, além dos efeitos de interações. Diante disso, o objetivo deste trabalho está centrado no estudo das funções estimáveis e das hipóteses testáveis em delineamentos ortogonais e parcialmente ortogonais com mais de dois fatores, à luz do procedimento GLM do sistema estatístico SAS. Face aos resultados obtidos, concluiu-se que para todos os efeitos principais, nos quais o subespaço gerado é individualmente ortogonal aos subespaços inerentes aos demais fatores, são estimáveis e, portanto, as hipóteses correspondentes são testáveis nos modelos sem interações. $\mathrm{Na}$ presença de interações, as funções estimáveis apresentam além de parâmetros do próprio fator, parâmetros das interações nas quais o fator está presente. Para esses casos, independente do modelo conter ou não conter interações, as hipóteses sobre médias ponderadas (tipo I) são equivalentes às hipóteses sobre médias ponderadas ajustadas (tipo II) e, como o termo completo é condição necessária para a ortogonalidade parcial ou plena, ocorre também a equivalência entre as hipóteses sobre médias não ponderadas ajustadas (tipos III e IV). A igualdade entre as hipóteses dos tipos I e II ocorre para todas as interações, nos delineamentos ortogonais e, nos delineamentos parcialmente 
ortogonais, ocorre para as interações formadas pelos fatores que não foram ortogonais entre si, pelas interações formadas por combinações de fatores que geraram subespaços individualmente ortogonais aos demais e para as todas as combinações formadas pelos fatores cujos subespaços não foram ortogonais entre si, além dos fatores cujos subespaços gerados foram individualmente ortogonais aos demais. Nessas interações as funções estimáveis apresentam parâmetros da própria interação, além de parâmetros das interações de grau maior que o grau da interação em estudo. Como uma aplicação imediata do conteúdo deste texto e, com o intuito de orientar os pesquisadores no tocante ao planejamento de experimentos ortogonais e parcialmente ortogonais, apresenta-se, ao final, um capitulo sobre o tema. 


\title{
ORTHOGONAL AND PARTIALLY ORTHOGONAL DESIGNS: THEORY AND APPLICATION
}

\author{
Author: DENISE GARCIA DE SANTANA
}

Adviser: Prof. ANTONIO FRANCISCO IEMMA

\section{SUMMARY}

In experimental situations, not rarely, the researcher faces the impossibility of planning balanced experiments. A serious problem arises immediately concerning the interpretation of the hypothesis tested with the statistical systems, mainly when there are several problems involved and a high unbalancing degree is present because, generally speaking, the hypothesis on the main effects of one of the factors contains the main effects of other effects, including the effects of the interactions. Taking that into account, the objective of this work is focused on the study of the estimable functions and of the testable hypothesis in orthogonal and partially orthogonal designs with more than two factors, based on the GLM procedure of the SAS statistical system. With the obtained results, one could conclude that for the main effects, in which the generated subspace is individually orthogonal to the inherent subspaces to the other factors, are estimable and, therefore, the corresponding hypothesis testable in models with no interactions. When interactions are present, the estimable functions present besides the parameters of the factor itself, parameters of the interactions in which the factor is present. In these cases, whether the model contains or not interactions, the hypothesis on weighted averages (type I) are equivalent to the hypothesis on proportional averages (type II) and, since the complete term is imperative to both full and partial orthogonalities, the equivalence between the hypothesis on non-proportional averages (types III and IV) also occurs. The equity between the hypothesis of the types I and II occurs in all interactions, in the orthogonal designs and, in the partially orthogonal designs, it occurs in the 
interactions formed by the factors that were not orthogonal between themselves, by the interactions formed by a combination of factors that generated subspaces individually orthogonal to the others and in all the combinations formed by the factors of which subspaces were not orthogonal between themselves, including the factors of which generated subspaces were individually orthogonal to the others. In these interactions the estimable functions present parameters of the interaction itself, including parameters of the interactions of higher degree than the degree of the interaction being studied. As an immediate application of the contents of this text and, with the aim of instructing the researchers concerning the planning of orthogonal and partially orthogonal experiments, a chapter on this subject can be found at the end of this abstract. 


\section{INTRODUÇÃO}

Um dos temas da estatistica experimental, especificamente em análise de variância, que tem motivado e preocupado os pesquisadores, consiste no estudo das hipóteses realmente testadas através do modelo adotado. De fato, a forma das hipóteses é diretamente dependente da estrutura do delineamento, que está associada entre outras caracteristicas, às freqüências das caselas. A distribuição das freqüências de maneira homogênea, que caracteriza os conjuntos completos e balanceados, é a mais discutida nos textos de estatística experimental e os modelos oriundos de tais conjuntos, apresentam as hipóteses mais simples de serem interpretadas. Entretanto, conjuntos balanceados podem ser, em muitos casos, uma exceção, pois a perda de parcelas por causas naturais ou acidentais, bem como a impossibilidade física de instalação de conjuntos balanceados constituem situações usuais na experimentação agronômica.

Sabe-se que a utilização de conjuntos desbalanceados afeta a interpretação prática dos resultados, no sentido de que as hipóteses de interesse do pesquisador que, em geral, são aquelas que envolvem apenas funções de um mesmo parâmetro, podem não ser testáveis. Ainda assim, muitos delineamentos de conjuntos desbalanceados ou mesmo incompletos foram, e ainda têm sido interpretados como se fossem balanceados. Naturalmente, tal procedimento pode gerar conclusões errôneas e afirmações que não serão reprodutiveis em ensaios futuros.

Nesse contexto, quando se pensou que a análise de variância era um tema bastante explorado, novos textos de estatistica experimental (Azaïs, 1994; Iemma, 1995; Claustriaux \& Iemma, 1999; Iemma \& Claustriaux, 1999) surgiram com o enfoque principal no estudo das hipóteses do modelo.

Para o estudo de tais hipóteses, conceitos como os de delineamentos ortogonais e não ortogonais, assim como os de conjuntos 
balanceados e desbalanceados, passaram a ser fundamentais. As primeiras idéias sobre delineamentos ortogonais foram apresentadas por Yates ${ }^{1}$ (1933) citado por Preece (1977), onde o autor utiliza o termo ortogonal para indicar aqueles delineamentos cujas médias não eram afetadas pelas diferenças entre blocos. Posteriormente, modernizada e formalizada por Pearce (1970), a idéia de ortogonalidade ficou restrita aos modelos de classificação dupla ou de blocos casualizados de conjuntos balanceados, necessitando ser ampliada para modelos mais gerais envolvendo vários fatores e interações.

A grande vantagem dos delineamentos ortogonais consiste no fato de que as funções clássicas envolvendo niveis de apenas um dos fatores são estimáveis e portanto testáveis no modelo sem interação, sejam eles associados a conjuntos balanceados ou desbalanceados. Em relação aos tipos de hipóteses, a ortogonalidade garante a igualdade entre os tipos I e II, ou seja, as hipóteses construídas como funções de médias ponderadas são iguais às hipóteses construidas como funções de médias ponderadas ajustadas, para os efeitos principais e interações. Tal caracteristica facilita de maneira significativa a utilização de programas computacionais para análise estatística. Essas vantagens se estendem aos delineamentos parcialmente ortogonais, pois a condição de ortogonalidade, para algumas combinações de fatores, possibilita a mesma igualdade de hipóteses dos tipos I e II para alguns efeitos principais e interações. Acrescenta-se aos delineamentos parcialmente ortogonais uma maior facilidade de planejamento pela não restrição de todas as combinações entre fatores apresentarem freqüências proporcionais.

Diante do exposto, este texto tem por objetivos:

- definir delineamentos ortogonais e parcialmente ortogonais para qualquer número de fatores do modelo;

1 Yates, F. The principles of orthogonality and confunding in replicated experiments. Journal of Agricukural Science, v.23, p.108-145, 1933. 
- apresentar a forma geral das hipóteses sobre os efeitos principais e interações para os delineamentos ortogonais, assim como, estabelecer propriedades sob ortogonalidade;

- apresentar a forma geral das hipóteses sobre os efeitos principais e interações para os delineamentos parcialmente ortogonais, assim como, estabelecer propriedades sob ortogonalidade parcial;

- obter um critério para determinação das freqüências de caselas de delineamentos ortogonais e parcialmente ortogonais. 


\section{REVISÃO DE LITERATURA}

\section{1 Conceitos e definições}

A primeira referência consistente do termo ortogonal foi apresentada por Yates (1933), citado por Preece (1977). O autor define ortogonalidade como uma propriedade dos delineamentos que garante que as diferentes classes de efeitos, aos quais o material experimental está sujeito, podem ser isoladas e estimadas sem qualquer obstáculo. Entretanto, a definição de ortogonalidade de Yates (1933) não menciona especificamente o termo delineamento ortogonal.

Pearce (1953) apresenta a seguinte definição para a ortogonalidade: "Duas classificações são mutuamente ortogonais se os vários grupos de parcelas formadas por uma classificação, são compostos de um número de parcelas proporcionais à outra classificação. Quando todos os pares de classificações são mutuamente ortogonais, o delineamento é dito ortogonal."

Preece (1977) faz uma revisão sobre os termos ortogonalidade e delineamento e mostra a confusão na literatura gerada pelos diferentes significados dos termos, principalmente entre os estatisticos e os chamados “combinatoristas". O autor comenta, também, que a definição de delineamento ortogonal apresentada por Pearce (1953) deve ser ampliada, principalmente quando aplicada a experimentos fatoriais na presença de interações. Para justificar o comentário, o autor utiliza um exemplo de Yates (1935) de um delineamento com três fatores, onde o fator $A$ apresenta três niveis $(0,1$ e 2$)$, o fator $B$, dois niveis $(0$ e 1$)$ e o fator $C$ dois niveis $(0$ e 1$)$. O esquema está descrito no Quadro 1. 
Quadro 1. Exemplo de Yates (1935) discutido por Preece (1977).

\begin{tabular}{|ccc|ccc|ccc|ccc|ccc|ccc|}
\hline \multicolumn{2}{|c|}{ Bloco1 } & \multicolumn{3}{|c|}{ Bloco 2 } & \multicolumn{3}{|c|}{ Bloco 3 } & \multicolumn{3}{|c|}{ Bloco 4 } & \multicolumn{3}{|c|}{ Bloco 5 } & \multicolumn{3}{|c|}{ Bloco 6 } \\
$A$ & $B$ & $C$ & $A$ & $B$ & $C$ & $A$ & $B$ & $C$ & $A$ & $B$ & $C$ & $A$ & $B$ & $C$ & $A$ & $B$ & $C$ \\
\hline 0 & 0 & 1 & 0 & 0 & 0 & 0 & 0 & 0 & 0 & 0 & 1 & 0 & 0 & 0 & 0 & 0 & 1 \\
0 & 1 & 0 & 0 & 1 & 1 & 0 & 1 & 1 & 0 & 1 & 0 & 0 & 1 & 1 & 0 & 1 & 0 \\
1 & 0 & 0 & 1 & 0 & 1 & 1 & 0 & 1 & 1 & 0 & 0 & 1 & 0 & 0 & 1 & 0 & 1 \\
1 & 1 & 1 & 1 & 1 & 0 & 1 & 1 & 0 & 1 & 1 & 1 & 1 & 1 & 1 & 1 & 1 & 0 \\
2 & 0 & 0 & 2 & 0 & 1 & 2 & 0 & 0 & 2 & 0 & 1 & 2 & 0 & 1 & 2 & 0 & 0 \\
2 & 1 & 1 & 2 & 1 & 0 & 2 & 1 & 1 & 2 & 1 & 0 & 2 & 1 & 0 & 2 & 1 & 1 \\
\hline
\end{tabular}

Fonte: Preece (1977).

Verificando os três fatores principais $A, B$ e $C$, observa-se que eles são mutuamente ortogonais, segundo a definição de Pearce (1953), pois as freqüências dos fatores dois a dois são proporcionais. Além disso, há um balanceamento entre as freqüências geradas pelos três fatores (Quadro 2).

Assim, segundo a definição de Pearce (1953) esse delineamento poderia ser caracterizado como ortogonal, uma vez que os fatores são mutuamente ortogonais e cada fator é ortogonal ao bloco.

Quadro 2. Ortogonalidade segundo a definição de Pearce (1953).

\begin{tabular}{|l|l|l|l|l|}
\cline { 2 - 5 } \multicolumn{1}{c|}{} & \multicolumn{2}{c|}{$B=0$} & \multicolumn{2}{c|}{$B=1$} \\
\cline { 2 - 5 } \multicolumn{1}{c|}{} & $C=0$ & $C=1$ & $C=0$ & $C=1$ \\
\hline$A=0$ & 3 & 3 & 3 & 3 \\
\hline$A=1$ & 3 & 3 & 3 & 3 \\
\hline$A=2$ & 3 & 3 & 3 & 3 \\
\hline
\end{tabular}

Fonte: Preece (1977).

\begin{tabular}{|c|c|c|}
\cline { 2 - 3 } \multicolumn{1}{c|}{} & $B=0$ & $B=1$ \\
\hline$A=0$ & 6 & 6 \\
\hline$A=1$ & 6 & 6 \\
\hline$A=2$ & 6 & 6 \\
\hline
\end{tabular}

\begin{tabular}{|c|c|c|}
\cline { 2 - 3 } \multicolumn{1}{c|}{} & $C=0$ & $C=1$ \\
\hline$A=0$ & 6 & 6 \\
\hline$A=1$ & 6 & 6 \\
\hline$A=2$ & 6 & 6 \\
\hline
\end{tabular}

\begin{tabular}{|c|c|c|}
\cline { 2 - 3 } \multicolumn{1}{c|}{} & $C=0$ & $C=1$ \\
\hline$B=0$ & 9 & 9 \\
\hline$B=1$ & 9 & 9 \\
\hline
\end{tabular}

No entanto, observando-se a combinação dos fatores dois a dois dentro dos blocos, observa-se que os efeitos principais $A$ e $B$, na interação $A B$ são ortogonais aos blocos, mas os fatores principais $B$ e $C$ na interação $B C$ não são ortogonais, pois não há proporcionalidade entre as freqüências de caselas, consequentemente a interação $A B C$ também não é ortogonal aos blocos. Isso é mostrado no Quadro 3, com as freqüências de caselas dentro de cada bloco, entre os fatores $A$ e $B$ e os fatores $B$ e $C$. $O$ caso $a$ mostra as freqüências das caselas dentro de cada bloco para os fatores $A$ e $B$, e a proporcionalidade entre 
as freqüências de caselas, o que não ocorre para o caso $b$ para os fatores $B$ e $C$ em relação aos blocos.

Quadro 3. Ortogonalidade parcial, segundo as idéias de Preece (1977).

a Parte ortogonal

\begin{tabular}{|c|c|c|}
\hline \multicolumn{3}{|c|}{ Bloco 1} \\
\hline & $B=0$ & $B=1$ \\
\hline$A=0$ & 1 & 1 \\
\hline$A=1$ & 1 & 1 \\
\hline$A=2$ & 1 & 1 \\
\hline
\end{tabular}

Bloco 2

\begin{tabular}{|c|c|c|}
\cline { 2 - 3 } \multicolumn{1}{c|}{} & $B=0$ & $B=1$ \\
\hline$A=0$ & 1 & 1 \\
\hline$A=1$ & 1 & 1 \\
\hline$A=2$ & 1 & 1 \\
\hline
\end{tabular}

Bloco 4

\begin{tabular}{|c|c|c|}
\cline { 2 - 3 } \multicolumn{1}{c|}{} & $B=0$ & $B=1$ \\
\hline$A=0$ & 1 & 1 \\
\hline$A=1$ & 1 & 1 \\
\hline$A=2$ & 1 & 1 \\
\hline
\end{tabular}

Bloco 5

\begin{tabular}{|c|c|c|}
\cline { 2 - 3 } \multicolumn{1}{c|}{} & $B=0$ & $B=1$ \\
\hline$A=0$ & 1 & 1 \\
\hline$A=1$ & 1 & 1 \\
\hline$A=2$ & 1 & 1 \\
\hline
\end{tabular}

Bloco 3

\begin{tabular}{|c|c|c|}
\cline { 2 - 3 } \multicolumn{1}{c|}{} & $B=0$ & $B=1$ \\
\hline$A=0$ & 1 & 1 \\
\hline$A=1$ & 1 & 1 \\
\hline$A=2$ & 1 & 1 \\
\hline
\end{tabular}

Bloco 6

\begin{tabular}{|c|c|c|}
\cline { 2 - 3 } \multicolumn{1}{c|}{} & $B=0$ & $B=1$ \\
\hline$A=0$ & 1 & 1 \\
\hline$A=1$ & 1 & 1 \\
\hline$A=2$ & 1 & 1 \\
\hline
\end{tabular}

$b$ Parte não ortogonal

\begin{tabular}{|c|c|c|}
\hline \multicolumn{3}{|c|}{ Bloc } \\
\hline & $B=0$ & $B=1$ \\
\hline$C=0$ & 2 & 1 \\
\hline$C=1$ & 1 & 2 \\
\hline
\end{tabular}

Bloco 4

\begin{tabular}{|c|c|c|}
\cline { 2 - 3 } \multicolumn{1}{c|}{} & $B=0$ & $B=1$ \\
\hline$C=0$ & 1 & 2 \\
\hline$C=1$ & 2 & 1 \\
\hline
\end{tabular}

Bloco 2

\begin{tabular}{|c|c|c|}
\cline { 2 - 3 } \multicolumn{1}{c|}{} & $B=0$ & $B=1$ \\
\hline$C=0$ & 1 & 2 \\
\hline$C=1$ & 2 & 1 \\
\hline
\end{tabular}

Bloco 5

\begin{tabular}{|c|c|c|}
\cline { 2 - 3 } \multicolumn{1}{c|}{} & $B=0$ & $B=1$ \\
\hline$C=0$ & 2 & 1 \\
\hline$C=1$ & 1 & 2 \\
\hline
\end{tabular}

Bloco 3

\begin{tabular}{|c|c|c|}
\cline { 2 - 3 } \multicolumn{1}{c|}{} & $B=0$ & $B=1$ \\
\hline$C=0$ & 2 & 1 \\
\hline$C=1$ & 1 & 2 \\
\hline
\end{tabular}

Bloco 6

\begin{tabular}{|c|c|c|}
\cline { 2 - 3 } \multicolumn{1}{c|}{} & $B=0$ & $B=1$ \\
\hline$C=0$ & 1 & 2 \\
\hline$C=1$ & 2 & 1 \\
\hline
\end{tabular}

O que foi constatado no artigo de Preece (1977) é que há uma diferença entre delineamentos ortogonais e delineamentos onde apenas parte da estrutura é ortogonal. No exemplo dado, apenas os efeitos principais são ortogonais entre si.

Este tipo de delineamento, ou seja, com ortogonalidade entre efeitos principais, foi discutido também por Addelman (1962). O autor mostra que é possivel ocorrer a ortogonalidade entre efeitos principais nos delineamentos com vários fatores $\mathrm{e}$, por conseguinte, a estimação não viesada dos efeitos principais quando a interação não está presente. Entretanto, o 
compromisso de se pressupor que não há interação para que as estimativas sejam não viesadas dificulta a modelagem e a interpretação dos resultados.

Claustriaux \& Iemma (1999) generalizam a discussão de Preece (1977) incluindo uma discussão sobre os termos completo, ortogonal e balanceado em análise de variância, uma vez que esta trilogia parece confundir autores e pesquisadores. Os autores focalizam a definição de delineamentos ortogonais para modelos com dois fatores, com e sem interação, ampliando as definições anteriores que se fixavam em modelos sem interação. Mostram, ainda, a característica principal de um delineamento ortogonal que é apresentar hipóteses do tipo I iguais às hipóteses do tipo II, provando a propriedade de que sob ortogonalidade, médias ponderadas são iguais a médias ponderadas ajustadas. Esta característica já havia sido discutida sobre um outro enfoque por Yates (1933) citado por Pearce (1963;1983) mostrando que num delineamento ortogonal as médias de tratamentos não eram afetadas pelas diferenças entre blocos. Em termos de hipóteses isso significa que, as funções estimáveis para os efeitos de tratamentos não contêm parâmetros dos blocos.

Azaïs (1994) faz um amplo comentário sobre modelos ortogonais e não ortogonais, mostrando o que o autor chama de decomposições do tipo I, II, III e IV, além de outros tipos de decomposições. O autor também apresenta, de maneira didática, o que acontece com as decomposições quando o número de observações por casela não é constante ou mesmo quando há casela vazia.

Iemma \& Claustriaux (1999) enfatizam a nomenclatura, e propiciam uma ampla discussão sobre a análise de variância a dois fatores de classificação com delineamentos ortogonais e não ortogonais, enfocando as funções estimáveis dos tipos I, II, III e IV fornecidas pelos sistemas SAS (X, 1990) e Minitab (X, 1996). Uma grande contribuição do artigo consiste na apresentação de uma figura resumo (Figura 1), contendo tipos de hipóteses associadas às estruturas dos delineamentos e dos dados. 


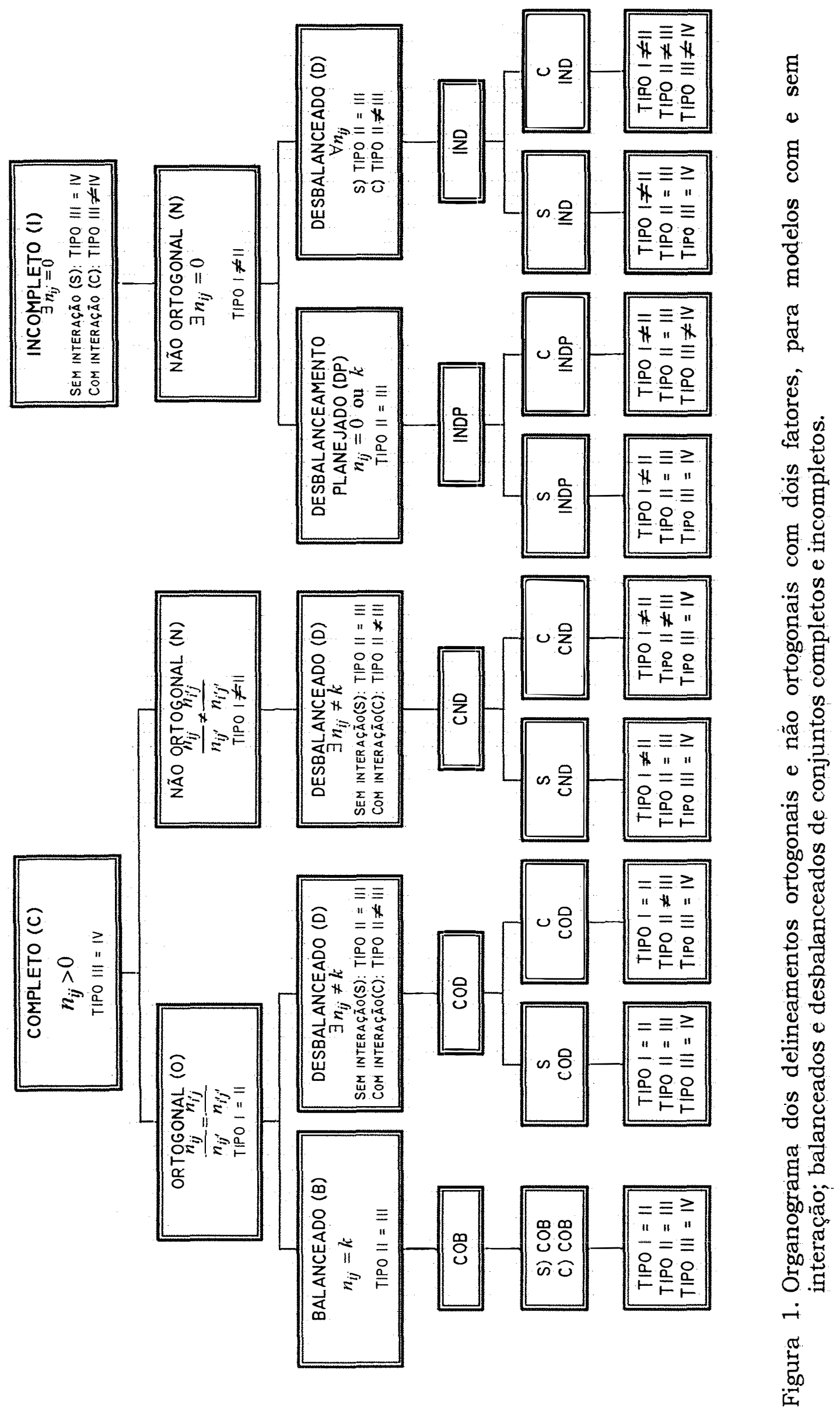




\subsection{Funções estimáveis e hịpóteses testáveis}

No planejamento de delineamentos experimentais é importante que o pesquisador defina, antecipadamente, quais são as hipóteses de maior interesse. Na verdade, conforme a relação modelo-dados, muitas das hipóteses de interesse em sua pesquisa podem não ser testáveis. Para que uma hipótese seja testável é necessário que a função paramétrica que a descreve seja estimável. Segundo Rao (1945) uma função linear paramétrica $\lambda^{\prime} \theta$ é dita estimável no modelo $y=X \theta+e$, Gauss Markov normal ordinário, se e somente se, existe uma combinação linear das observações, $a^{\prime} y$, tal que $E\left[a^{\prime} y\right]=\lambda^{\prime} \theta$ (Iemma, 1990).

Se $\lambda^{\prime} \theta$ é estimável, ela apresenta vários estimadores não viesados, dentro os quais um único é classificado como melhor. Seja o modelo de GaussMarkov e sejam as possiveis combinações $a^{\prime} y$ tais que: $E\left[a^{\prime} y\right]=\lambda^{\prime} \theta$. Então $a^{*} y$ é o melhor estimador não viesado de $\lambda^{\prime} \theta$, se e somente se, $\operatorname{var}\left[a^{\star} y\right]=\min \operatorname{var}\left[a^{\prime} y\right], \quad \forall a^{\prime} y: E\left[a^{\prime} y\right]=\lambda^{\prime} \theta$. Nesse contexto, segundo o teorema de Gauss-Markov, seu BLUE é obtido de modo único, através de $\lambda^{\prime} \theta=\lambda^{\prime} \theta^{\circ}$, onde $\theta^{\circ}$ é qualquer solução das equações normais. Sua variância é dada por: $\operatorname{var}\left[\lambda^{\prime} \theta\right]=\operatorname{var}\left[\lambda^{\prime} \theta^{\circ}\right]=\lambda^{\prime}\left(X^{\prime} X\right)^{G} \lambda \sigma^{2}$.

A definição apresentada por Rao (1945) tem grande importância nas demonstrações teóricas, mas tem de pouco valor prático, uma vez que depende da dimensão dos dados. Isto levou ao estabelecimento de regras práticas de estimabilidade. Dentre as regras de estimabilidade mais importantes estão as funções básicas estimáveis incorporadas pelo Proc GLM do SAS, que constrói uma forma geral para as funções estimáveis através das linhas não nulas de $L=\left(X^{\prime} X\right)^{g 2} X^{\prime} X$ (Mondardo, 1994). Assim, a forma geral apresentada pelo sistema SAS (X, 1990) das funções estimáveis envolve todos os parâmetros do modelo e à cada linha não nula, linearmente independente, de $\left(X^{\prime} X\right)^{g^{2}} X^{\prime} X$ é associado um símbolo L, de modo que o número de L's 
diferentes representa o posto máximo da hipótese testada. O procedimento GLM do sistema estatístico SAS tem como padrão construir a matriz L, utilizando como inversa generalizada de $X^{\prime} X$, a inversa reflexiva $g 2$. A matriz L é a mesma matriz H descrita por Iemma (1987).

No sistema SAS (X, 1990) são incorporados quatro tipos de funçōes estimáveis: funçōes sobre médias ponderadas não ajustadas (Tipo I), funçōes sobre médias ponderadas ajustadas (Tipo II), funçōes sobre médias não ponderadas ajustadas (Tipo III) e funçōes sobre médias não ponderadas ajustadas, segundo as caselas vazias (Tipo IV) (Iemma, 1991).

A escolha das funçōes estimáveis do tipo I para os efeitos principais pode envolver parâmetros do outro fator quando na presença de caselas vazias ou mesmo em conjuntos completos não ortogonais de modelos sem interação, além de parâmetros da interação nos modelos com interação. Essa característica dificulta sobremaneira a interpretação dos resultados pelo pesquisador. Já as funçōes estimáveis do tipo II por serem ajustadas para o outro fator, podem testar hipóteses de maior interesse do pesquisador quando não há caselas vazias. As funçōes estimáveis do tipo III são importantes no estudo de modelos com interação, mas quando o número de caselas vazias e o número de niveis do fator aumenta, elas podem ser complexas e mesmo fugir do interesse do pesquisador (Mondardo, 1994).

Diante disso, Camarinha Filho (1995) discute que é indispensável que os manuais de pacotes estatísticos sejam mais esclarecedores, minimizando os erro de interpretaçōes das hipóteses na análise dos dados. Acrescenta Santos (1994) a importância da interpretação correta das hipóteses devido a grande diversidade de programas computacionais, principalmente na presença de caselas vazias.

Aliado ao problema da interpretação das hipóteses está a escolha do modelo. Os modelos superparametrizados, conhecidos como modelo-S são de grande interesse pois mostram explicitamente os parâmetros. Entretanto, segundo Hocking (1985), Searle (1987) e Iemma (1989;1991) entre outros, esses modelos apresentam o inconveniente de apresentar mais parâmetros do que médias de caselas. Diante do problema, o modelo de média de caselas 
descrito em Speed, Hocking \& Hackney (1978), conhecido como modelo-M, é uma alternativa ao problema do excesso de parâmetros, apresentando ainda a vantagem de descrever as hipóteses com grande facilidade.

\subsection{Delineamentos ortogonais}

Pearce (1970) formaliza os delineamentos ortogonais no seu caso mais geral, onde a ortogonalidade é caracterizada pela independência da média ajustada de tratamentos, dos efeitos dos blocos. As considerações do autor iniciam com a utilização da notação apresentada por Tocher (1952) que, utilizando a teoria dos delineamentos em blocos incompletos, define uma matriz de incidência $\boldsymbol{n}$, cujos elementos são formados pelo número de vezes em que cada tratamento ocorre em cada bloco e define $\mathrm{x}^{\alpha \delta}$ como uma matriz diagonal com elementos, $x_{1}^{\alpha}, x_{2}^{\alpha}, \ldots$, onde $x_{1}, x_{2}, \ldots$, são os elementos do vetor $x$. A seguir, o autor utiliza a notação apresentada por Jones (1959), que trabalha com duas matrizes de delineamento, a matriz $D$ referente aos blocos e a matriz $\Delta$ referente aos tratamentos. Então, $n=\Delta D^{\prime}, k^{\delta}=D D^{\prime}, r^{\delta}=\Delta \Delta^{\prime}, D 1=k, \Delta 1=r$ e $D^{\prime} 1=1=\Delta^{\prime} 1$. Se os dados são descritos através do vetor $y$, o vetor dos totais de blocos é $B=D y$ e o vetor de totais de tratamentos é $T=\Delta y$. O total geral $G$ é dado tanto por $B^{\prime} 1$ como por $T^{\prime \prime} 1$ e a média geral é $m=G / N$, onde $N$ é o número total de observações.

Tocher (1952) mostra a importãncia da matriz $\Omega$ definida como:

$$
\Omega^{-1}=\left(r^{\delta}-n k^{-\delta} n^{\prime}+r r^{\prime} / N\right)^{-1}
$$

sendo $\Omega^{-1}$ uma inversa clássica de $\Omega$ sob a restrição $r^{\prime} \hat{\tau}=0$.

Consequentemente ,

$$
Q=T-n k^{-\delta} B
$$

onde $Q$ é o vetor $v \times 1$ dos totais ajustados de tratamentos. Então, o vetor de efeitos de tratamentos $\gamma$ é estimado por $\Omega Q$. Se a média geral $m$ é 
acrescentada em cada elemento, o resultado é o vetor de médias ajustadas de tratamentos $\boldsymbol{\alpha}$, dado por

$$
a=m 1+\Omega Q
$$

Assim, tem-se a matriz de covariâncias

$$
\Omega \hat{\sigma}^{2}
$$

onde $\hat{\sigma}^{2}$ é o quadrado médio do resíduo da análise de variância. A soma de quadrados de tratamentos é dada por:

$$
Q^{\prime} \Omega Q
$$

Segundo Pearce (1970), se o delineamento é ortogonal, todos elementos da diagonal de $\Omega \mathrm{e}$, consequentemente, $\Omega^{-1}=r^{\delta}$ e $n k^{-\delta} n^{\prime}-r r^{\prime} / N$ são iguais a zero.

Generalizando, o i-ésimo elemento da diagonal de $n k^{-\delta} n^{\prime}-r r^{\prime} / N$ é

$$
\sum_{i}\left(n_{i j}^{2} / k_{j}\right)-r_{i}^{2} / N=\sum_{i}\left\{\left(n_{i j}-r_{i} k_{j} / N\right)^{2} / k_{j}\right\}=0
$$

Entretanto, para todos $i \mathrm{e} j, n_{i j}=r_{i} k_{j} / N$, ou seja,

$$
n=\frac{r k^{\prime}}{N}
$$

sendo descrita como condição de ortogonalidade, onde $k$ é vetor que contém as freqüências de blocos.

Segundo o autor, isso significa que todos os blocos são construídos proporcionalmente, da mesma maneira, com relação aos tratamentos e todos os tratamentos são distribuidos proporcionalmente entre os blocos. Substituindo (6) em (1), chega-se a:

$$
\Omega=r^{-\delta}
$$

isto ê, as médias ajustadas de tratamentos são independentemente distribuidas.

Outro aspecto importante dos delineamentos ortogonais, segundo Pearce (1970), é que nestes, todos os contrastes são estimados com a mesma eficiência. Isto não significa que delineamentos não ortogonais sejam menos 
eficientes, como é o caso dos desbalanceamentos planejados, que apesar de serem formados por conjuntos incompletos como os blocos incompletos balanceados, os latices e os quadrados latinos, possuem variâncias constantes nos contrastes simples entre dois niveis de um mesmo fator (Claustriaux \& Iemma, 1999).

O estudo dos delineamentos ortogonais com dois fatores (Iemma \& Claustriaux, 1999) e três fatores (Iemma \& Santana, 2000) de modelos com e sem interações, ampliou a própria definição, que anteriormente era restrita a modelos com dois fatores sem interação, e enfatizou a propriedade dos delineamentos ortogonais de apresentarem hipóteses do tipo I iguais ás hipóteses do tipo II. Uma prova dessa igualdade para modelos com dois fatores foi apresentada por Christensen (1987). Utilizando a notação $R$ (.), o autor mostrou que para o modelo caracterizado por $y_{i j k}=\mu+\alpha_{i}+\beta_{j}+e_{i j k}$ oriundo de um delineamento ortogonal, a hipótese do tipo I é igual a hipótese do tipo II para qualquer fator do modelo e, portanto, $R(\alpha \mid \mu)=R(\alpha \mid \mu, \beta) \quad$ e $R(\beta \mid \mu)=R(\beta \mid \mu, \alpha)$.

A igualdade $R(\beta \mid \mu)=R(\beta \mid \mu, \alpha)$ ocorre para todo $y$, se e somente se $C\left(P_{\mu, \beta}-1 / n J J^{\prime}\right) \perp C\left(P_{\mu, \alpha}-1 / n J J^{\prime}\right)$, ou seja, $\left(P_{\mu, \beta}-1 / n J J^{\prime}\right)\left(P_{\mu, \alpha}-1 / \pi J J^{\prime}\right)=\phi$. Sendo $\quad R(\beta \mid \mu)=y^{\prime}\left(P_{\mu, \beta}-1 / n J J^{\prime}\right) y \quad$ e $\quad R(\beta \mid \mu, \alpha)=y^{\prime}\left(P-P_{\mu, \alpha}\right) y \quad$ é suficiente mostrar que

$$
\left(P_{\mu, \beta}-1 / n J J^{\prime}\right)=\left(P-P_{\mu, \alpha}\right) \Leftrightarrow\left(P_{\mu, \beta}-1 / n J J^{\prime}\right)\left(P_{\mu, \alpha}-1 / n J J^{\prime}\right)=\phi
$$

Outra maneira de verificar a ortogonalidade, sem a utilização da notação $R$ (.) é obtida através da utilização do método de ortogonalização de Gram-Schmidt que segundo Christensen (1987) consiste em tornar todas as colunas de $\alpha$ e $\beta$ ortogonais a J. Se as colunas transformadas $\alpha$ são ortogonais as colunas transformadas de $\beta$ então $R(\beta \mid \mu)=R(\beta \mid \mu, \alpha)$ e pela simetria $R(\alpha \mid \mu)=R(\alpha \mid \mu, \beta)$. Em Iemma (1990) este algoritmo está descrito em detalhes. 
Para modelos com três fatores Iemma \& Santana (2000) mostram que a ortogonalidade é obtida quando combinações dos fatores dois a dois apresentam freqüências de caselas proporcionais, sendo estas proporcionalidades para um modelo com três fatores caracterizado por: $y_{i j k l}=\mu+\alpha_{i}+\beta_{j}+\gamma_{k}+e_{i j k l}$ com $i=1, \ldots, a$ niveis do fator $A ; j=1, \ldots, b$ niveis do fator $B ; k=1, \ldots, c$ niveis do fator $C$ e $l=1, \ldots, n_{i j k}$ observações na casela $(i ; j ; \mathrm{k})$ as proporcionalidades dadas por:

$$
\begin{gathered}
\frac{n_{i j .}}{n_{i j^{\prime} .}}=\frac{n_{i^{\prime} j .}}{n_{i^{\prime} j^{\prime} .}}, \forall(i \neq i ; j \neq j) ; \quad \frac{n_{i . k}}{n_{i . k^{\prime}}}=\frac{n_{i^{\prime} . k}}{n_{i^{\prime} \cdot k^{\prime}}} \forall\left(i \neq i^{\prime}, k \neq k\right) \\
\frac{n \cdot k_{j}}{n_{\cdot j k^{\prime}}}=\frac{n:^{\prime} k J}{n_{\cdot j^{\prime} k^{\prime}}} \rightarrow,\left(j \neq j^{\prime}, k \neq k^{\prime}\right)
\end{gathered}
$$

Os autores mostraram ainda, que dada a ortogonalidade, as somas de quadrados associadas as hipóteses dos tipos I e II são iguais para todos os efeitos principais e interações do modelo, e que funções de um mesmo parâmetro são estimáveis num modelo sem interações. Além disso, em decorrência da ortogonalidade, ocorre a igualdade entre todas as decomposições das somas de quadrados para cada efeito principal e interação.

\subsection{Delineamentos parcialmente ortogonais}

Pearce (1983) mostra que a condição de ortogonalidade proposta por Pearce (1970) dada por $n=\frac{r k^{\prime}}{N}$ e pode não ser aplicada para todos os tratamentos, mas para apenas um grupo de tratamentos. Neste caso,

$$
\mathrm{N}=\frac{1}{n}\left[\frac{\rho k^{\prime}}{M}\right], \quad r=\mathrm{N} 1_{b}=\left[\frac{\rho}{\rho_{o}}\right]
$$


onde $\rho$ é o vetor de repetições dos tratamentos dispostos ortogonalmente e $M$ é arbitrário $\left(M 1_{b} / n=\rho_{o}\right)$. Então,

$$
\Omega^{-1}=\left[\begin{array}{c:c}
\rho^{\delta} & 0^{\prime} \\
\hdashline 0 & \rho_{o}^{\delta}-M k^{-\delta} M^{-1} / n^{2}+\rho_{o} \rho_{o}^{\prime} / n
\end{array}\right]
$$

Isto mostra que os contrastes podem ser estimados com a maior eficiência se for considerada apenas a parte na qual a ortogonalidade foi alcançada. Em alguns casos é útil isolar o componente não ortogonal do delineamento. Escrevendo $\Omega^{-1}$ como:

$$
\Omega^{-1}=r^{\delta}-\mathrm{N} k^{-\delta} \mathrm{N}^{\prime}+r r^{\prime} / n,
$$

então,

$$
\Omega^{-1}=r^{\delta}-A k^{-\delta} A^{\prime}
$$

onde:

$$
A=\mathrm{N}-r k^{\prime} / n
$$

é uma matriz definida como a matriz de não ortogonalidade (Pearce 1970).

As idéias de estruturas parcialmente ortogonais foram largamente apresentadas e discutidas por Finney (1945a, 1945b, 1946a, 1946b) em seus estudos sobre as propriedades ortogonais de delineamentos em quadrados latinos, $4 \times 4,5 \times 5$ e $6 \times 6$, nos quais mostra a importância da ortogonalidade para o estudo de efeitos principais no modelo.

Como aconteceu com os delineamentos ortogonais, a teoria de delineamentos parcialmente ortogonais esteve centrada nos modelos com dois fatores, sem interação. Santana \& Iemma (2000) estudando os delineamentos parcialmente ortogonais com três ou mais fatores, mostraram que quando até $K$-2 fatores, onde $K$ é o número de fatores, apresentam subespaços gerados individualmente ortogonais aos demais fatores, a igualdade entre as hipóteses do tipo I e II é mantida para esse grupo de fatores e para a interação formada pela combinação de fatores cujos subespaços não foram ortogonais entre si, além de outras interações quando o modelos tem mais de três fatores. 
Santana \& Iemma (2000) apresentaram diagramas mostrando os casos de ortogonalidade parcial para modelos com três ou mais fatores que são referências para o planejamento de experimentos onde o interesse do pesquisador está centrado em apenas alguns parâmetros e interações e portanto, não se faz necessário que todas as combinações de fatores dois a dois apresentem freqüências proporcionais nas caselas. 


\section{MATERIAL E MÉTODOS}

\section{1 Material}

Os exemplos utilizados nesse estudo, fazem parte do ensaio instalado sobre canafistula (Pelthophorum dubium Spreng) e conduzido no Laboratório de Análise de Sementes da Escola Superior de Agricultura "Luiz de Queiroz" -USP, com o objetivo de ilustrar os resultados aqui obtidos. O experimento constou de quatro fatores: substrato (rolo de papel e caixa "gerbox"), temperatura $\left(25\right.$ e $\left.30^{\circ} \mathrm{C}\right)$, dormência (com e sem quebra de dormência) e época de avaliação (5 e 10 dias), sendo que as freqüências das caselas e o número de fatores utilizados em cada exemplo foram determinados em função do delineamento: ortogonal (balanceado ou desbalanceado) ou parcialmente ortogonal, com cada observação composta por 50 sementes de canafistula .

\subsection{Métodos}

A notação básica utilizada nesse estudo é aquela apresentada por Iemma \& Palm (1992), Iemma \& Claustriaux (1997; 1998) e Claustriaux \& Iemma (1999) sobre os delineamentos ortogonais com dois fatores, que foi o ponto de partida desta pesquisa. As definições e propriedades dos delineamentos ortogonais e parcialmente ortogonais foram sedimentadas, após estudos sobre as freqüências das caselas de modelos com dois, três e quatro fatores, na presença e ausência de interações. 


\subsubsection{O termo ortogonal na análise de variância}

Seja o modelo linear $y=X \theta+e$, onde $y$ é um vetor de dados, estocasticamente entendido como um vetor de realizaçōes de variáveis aleatórias; $X$ é uma matriz de delineamento, de posto completo ou incompleto; $\theta$ é um vetor de parâmetros desconhecidos e $e$ é um vetor de variáveis aleatórias nāo-observáveis.

Nesse contexto, o correspondente sistema de equaçōes lineares $y=X \theta$ é sabidamente inconsistente e uma alternativa para obter uma boa aproximação para $\theta$, consiste na utilização do método de minimos quadrados.

Ademais, o sistema de equaçōes normais $X^{\prime} X \theta=X^{\prime} y$, sempre consistente, fornece soluçōes aproximadas de mínimos quadrados, $\theta^{o}=\left(X^{\prime} X\right)^{G} X^{\prime} y$, para o sistema inconsistente $y=X \theta$, onde $\left(X^{\prime} X\right)^{G}$ é uma inversa generalizada qualquer de $X^{\prime} X$. Além disso, para qualquer $\theta^{\circ}$, solução das equações normais, a aproximação de mínimos quadrados $\hat{y}=X \theta^{\circ}$ para o vetor $y$, è invariante. Também, $\hat{y}=X \theta^{\circ}=P y$, ou seja, a aproximação de minimos quadrados para $y$, coincide com a projeção ortogonal de $y$ sobre o espaço gerado pelas colunas de $X, C(X)$ ( Iemma \& Palm, 1992).

Tem-se ainda, o erro devido à aproximação de mínimos quadrados, dado por $\hat{e}=y-\hat{y}=(I-P) y$, onde, $\hat{e}$ pertence ao complemento ortogonal do espaço coluna da matriz $X$. Assim, fica garantida a partição do vetor $y$ de observaçōes, na soma de dois vetores pertencentes a subespaços ortogonais: o vetor $\hat{y} \in C(X)$ e o vetor $\hat{e} \in C^{L}(X)$ (Figura 2). 


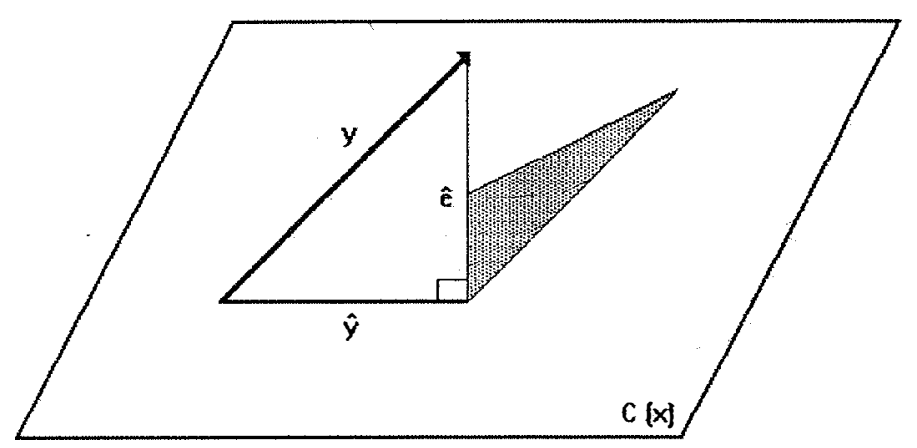

Figura 2. Decomposição ortogonal do vetor $y$ das observações.

Dada a ortogonalidade, aplica-se o Teorema de Pitágoras, obtendo-se a decomposição conhecida como análise de variância. Assim,

$$
\|y\|^{2}=\|\hat{y}\|^{2}+\|\hat{e}\|^{2}
$$

onde:

$\|y\|^{2}=\langle y, y\rangle=y^{\prime} y=$ Soma de quadrados total

$\|\hat{y}\|^{2}=\langle\hat{y}, \hat{y}\rangle=\hat{y}^{\prime} \hat{y}=y^{\prime} P y=$ Soma de quadrados de parâmetros

$\|\hat{e}\|^{2}=\langle\hat{e}, \hat{e}\rangle=\hat{e}^{\prime} \hat{e}=y^{\prime}(I-P) y=$ Soma de quadrados de erros

A primeira partição $\|y\|^{2}=\|\hat{y}\|^{2}+\|\hat{e}\|^{2}$ è sempre ortogonal, por construção, mas tem pouco valor prático (Iemma, Palm \& Claustriaux, 1993). A segunda partição, isto é, a decomposição da soma de quadrados de parâmetros, é que reflete os anseios do pesquisador e é sobre tal decomposição que foram inseridos os termos delineamento ortogonal e parcialmente ortogonal. 


\subsubsection{Delineamentos ortogonais}

\subsubsection{Modelos com dois fatores}

Definição 1. Segundo Azaïs (1994), um modelo com dois fatores cruzados sem interação, descrito por: $y_{i j k}=\mu+\alpha_{i}+\beta_{j}+e_{i j k} \operatorname{com} i=1, \ldots, a$ niveis do fator $A ; j=1, \ldots, b$ niveis do fator $B ; k=1, \ldots, n_{i j}$ observaçōes na casela $(i ; j)$, é dito ortogonal se o subespaço gerado pelas colunas de $X_{\alpha}$ (submatriz de $X$ referente ao fator $A$ ) após o ajuste de $\mu$, é ortogonal ao subespaço gerado pelas colunas de $X_{\beta}$ (submatriz de $X$ referente ao fator $B$ ), após o ajuste de $\mu$.

A matriz do modelo linear $y=X \theta+e$ apresenta, então, a seguinte partição:

$$
X=\left[\begin{array}{l:l:l}
X_{\mu} & X_{\alpha} & X_{\beta}
\end{array}\right]
$$

Tem-se um delineamento ortogonal se:

$$
C\left(X_{\alpha \mid \mu}\right) \perp C\left(X_{\beta \mid \mu}\right)
$$

onde:

$X_{\left.\alpha\right|_{\mu}}$ é a submatriz de $X$ que denota o ajuste de $\alpha$, após o ajuste de $\mu$;

$X_{\beta \mid \mu}$ é a submatriz de $X$ que denota o ajuste de $\beta$, após o ajuste de $\mu$.

Teorema 1. "Uma condição necessária e suficiente para que os subespaços, descritos na definição 1 , sejam ortogonais é que as freqüências das caselas sejam proporcionais" (Claustriaux \& Iemma, 1999).

$$
C\left(X_{\alpha \mid \mu}\right) \perp C\left(X_{\beta \mid \mu}\right) \Leftrightarrow \frac{n_{i j}}{n_{i j^{\prime}}}=\frac{n_{i^{\prime} j}}{n_{i^{\prime} j^{\prime}}} ; \quad \forall\left(i \neq i^{\prime}, j \neq j^{\prime}\right)
$$

A prova do teorema 1, pode ser obtida, utilizando-se de propriedades da álgebra de matrizes ou por indução. Em álgebra de matrizes, 
mostra-se que se o subespaço gerado pelas colunas de $X_{\alpha}$, após ajuste de $\mu$, é ortogonal ao subespaço gerado pelas colunas de $X_{\beta}$, após o ajuste de $\mu$, o produto dos respectivos projetores é nulo,

$$
\begin{gathered}
C\left(X_{\alpha \mid \mu}\right) \perp C\left(X_{\beta \mid \mu}\right) \Leftrightarrow P_{\alpha \mid \mu} P_{\beta \mid \mu}=\varnothing \\
\text { Sendo } P_{\alpha \mid \mu}=P_{\mu, \alpha}-P_{\mu} \text { e } P_{\beta \mid \mu}=P_{\mu, \beta}-P_{\mu}, \text { então, } \\
\left(P_{\mu, \alpha}-P_{\mu}\right)\left(P_{\mu, \beta}-P_{\mu}\right)=P_{\mu, \alpha} P_{\mu, \beta}-P_{\mu, \alpha} P_{\mu}-P_{\mu} P_{\mu, \beta}+P_{\mu} P_{\mu}=\varnothing
\end{gathered}
$$

onde as matrizes envolvidas são simétricas e idempotentes, por serem projetores ortogonais.

Além disso, se $C\left(X_{\mu}\right) \subset C\left(X_{\mu, \alpha}\right)$ e $C\left(X_{\mu}\right) \subset C\left(X_{\mu, \beta}\right)$, então:

$$
P_{\mu} P_{\mu, \alpha}=P_{\mu, \alpha} P_{\mu}=P_{\mu} \text { e } P_{\mu} P_{\mu, \beta}=P_{\mu, \beta} P_{\mu}=P_{\mu}
$$

Dada a idempotência de $P_{\mu}$, a expressão 1 passa a ser escrita como:

$$
P_{\alpha \mid \mu} P_{\beta \mid \mu}=P_{\mu, \alpha} P_{\mu, \beta}-P_{\mu}=\varnothing
$$

resultando assim, na seguinte condição de ortogonalidade,

$$
C\left(X_{\alpha \mid \mu}\right) \perp C\left(X_{\beta \mid \mu}\right) \Leftrightarrow P_{\mu, \alpha} P_{\mu, \beta}=P_{\mu}
$$

Para o modelo linear ordinário de Gauss-Markov $y=X \theta+e$, o projetor referente a $\mu$ é: $P_{\mu}=X_{\mu} X_{\mu}^{+}$, onde $X^{+}$é a inversa generalizada de Moore-Penrose de $X$. Uma representação de cada termo de $P_{\mu}$ é apresentada a seguir.

$$
P_{\mu}=\left(p_{i j k}\right) ; \quad p_{i j k}=\frac{1}{n_{. .}} ; \quad \forall(i, j, k)
$$


Sendo $P_{\mu, \alpha}=P_{\alpha}$ e $P_{\mu, \beta}=P_{\beta}$ (Iemma 1989) o produto $P_{\mu, \alpha} P_{\mu, \beta}$ passa a ser escrito da seguinte forma: $P_{\mu, \alpha} P_{\mu, \beta}=X_{\alpha} X_{\alpha}^{+} X_{\beta} X_{\beta}^{+}$. Como as matrizes $X_{\alpha}$ e $X_{\beta}$ são de posto coluna completo e, portanto, possuem inversa à esquerda, tem-se:

$$
P_{\mu, \alpha} P_{\mu, \beta}=X_{\alpha}\left(X_{\alpha}^{\prime} X_{\alpha}\right)^{-1} X_{\alpha}^{\prime} X_{\beta}\left(X_{\beta}^{\prime} X_{\beta}\right)^{-1} X_{\beta}^{\prime}
$$

Estudando-se a forma de algumas matrizes definidas em (3), temse as seguintes estruturas,

$$
\left(X_{\alpha}^{\prime} X_{\alpha}\right)^{-1}=\operatorname{diag}\left\{1 / n_{i .}\right\} ; \quad\left(X_{\beta}^{\prime} X_{\beta}\right)^{-1}=\operatorname{diag}\left\{1 / n_{. j}\right\}
$$

Como pode ser observado, a inversa clássica do produto $X_{\alpha}^{\prime} X_{\alpha}$ é uma matriz diagonal cujos elementos não nulos, correspondem aos inversos das freqüências de cada nivel do fator $A$, o mesmo acontecendo para o fator $B$. $O$ produto entre as matrizes devidas aos fatores $A$ e $B$ descrito por $X_{\alpha}^{\prime} X_{\beta}$, tem como resultado uma matriz cujos elementos são exatamente as freqüências das caselas de uma tabela de contingência de dupla entrada representada por:

$$
X_{\alpha}^{\prime} X_{\beta}=\left(x_{i j}\right), \quad x_{i j}=n_{i j}
$$

O produto matricial da expressão 3, é apresentado a seguir.

$$
P_{\mu, \alpha} P_{\mu, \beta}=X_{\alpha}\left[\begin{array}{cc}
1 / n_{i .} & 0 \\
0 & 1 / n_{i^{\prime}}
\end{array}\right]\left[\begin{array}{cc}
n_{i j} & n_{i j^{\prime}} \\
n_{i^{\prime} j} & n_{i j^{\prime}}
\end{array}\right]\left[\begin{array}{cc}
1 / n_{. j} & 0 \\
0 & 1 / n_{.^{\prime}}
\end{array}\right] X_{\beta}^{\prime}
$$

Efetuando-se o produto, obteve-se uma representação de cada termo da matriz definida em (3), 


$$
P_{\mu, \alpha} P_{\mu, \beta}=\left(p_{i j k}^{*}\right), \quad p_{i j k}^{*}=\frac{n_{i j}}{n_{i,} n_{, j}}
$$

Portanto, da condição de ortogonalidade em (2), tem-se a seguinte relação,

$$
\frac{n_{i j}}{n_{i .} n_{. j}}=\frac{1}{n_{. .}}
$$

Desse modo,

$$
C\left(X_{\alpha \mid \mu}\right) \perp C\left(X_{\beta \mid \mu}\right) \Leftrightarrow \frac{n_{i j}}{n_{i,} n_{. j}}=\frac{1}{n_{. .}}
$$

Então,

$$
n_{i j}=\frac{n_{i \cdot} n_{\cdot j}}{n_{\cdot \cdot}}
$$

é uma condição necessária e suficiente, em termos das freqüências das caselas, para a ortogonalidade dos dois subespaços de um modelo com dois fatores. Pode-se verificar também, que uma condição necessária e suficiente para $n_{i j}=\frac{n_{i .} n_{\cdot j}}{n_{. .}}$, é que as freqüências das caselas sejam proporcionais, isto é:

$$
\frac{n_{i j}}{n_{i j^{\prime}}}=\frac{n_{i^{\prime} j}}{n_{i^{\prime} j^{\prime}}}, \quad \forall(i \neq i, j \neq j)
$$

Segundo Iemma \& Claustriaux (1997), a proporcionalidade entre as freqüências de caselas, faz com que muitos autores utilizem os termos ortogonal e proporcional como sinônimos sem grandes problemas. Assim, uma simplificação, em termos da compreensão do pesquisador, do conceito de delineamentos ortogonais pode ser feita, enfocando-se diretamente os casos nos quais as freqüências das caselas são proporcionais. 


\subsubsection{Modelos com três fatores}

$\mathrm{O}$ estudo dos delineamentos ortogonais com três fatores é uma extensão dos trabalhos de Iemma \& Palm (1992), Iemma \& Claustriaux (1997, 1998) e Claustriaux \& Iemma (1999) sobre os delineamentos ortogonais com dois fatores, como dito anteriormente, bem como da definição apresentada por Pearce (1953). Nessa definição, o autor afirma que a ortogonalidade é garantida quando todos os pares de "classificaçōes" são mutuamente ortogonais. Fazendo uma ampliação dessa definição, isso implica que independente do número de fatores do modelo, a ortogonalidade é conseguida quando os fatores dois a dois são ortogonais.

As idéias de Pearce (1953) associadas às de Azaïs (1994), de Iemma \& Claustriaux (1997; 1998) e de Claustriaux \& Iemma (1999) motivaram a seguinte definição:

Definição 2. Um delineamento com três fatores cruzados sem interaçōes, caracterizado por: $y_{i j k l}=\mu+\alpha_{i}+\beta_{j}+\gamma_{k}+e_{i j k l}$ com $i=1, \ldots, a$ niveis do fator $A ; j=1, \ldots, b$ niveis do fator $B ; k=1, \ldots, c$ niveis do fator $C$ e $l=1, \ldots, n_{i j k}$ observaçōes na casela $(i ; j ; k)$, é dito ortogonal, se o subespaço gerado pelas colunas da submatriz de $X$ referentes a cada fator, após o ajuste de $\mu$, é individualmente ortogonal aos subespaços gerados pelas colunas das submatrizes de $X$ referentes aos outros dois fatores, após o ajuste de $\mu$.

A matriz do modelo linear $y=X \theta+e$ apresenta, então, a seguinte partição:

$$
X=\left[\begin{array}{l:l:l:l}
X_{\mu} & X_{\alpha} & X_{\beta} & X_{\gamma}
\end{array}\right]
$$

onde:

$X_{\mu}$ é a submatriz de $X$ referente a constante $\mu$;

$X_{\alpha}$ é a submatriz de $X$ referente ao fator $A$;

$X_{\beta}$ é a submatriz de $X$ referente ao fator $B$;

$X_{\gamma}$ é a submatriz de $X$ referente ao fator $C$. 
E, de modo análogo à definição 1 obtém-se: $X_{\alpha \mid \mu}, X_{\beta \mid \mu} \mathrm{e} X_{\gamma \mid \mu}$.

Teorema 2. "Uma condição necessária e suficiente para que um delineamento com $K$ fatores $(K \geq 2)$ seja ortogonal é que as freqüências das caselas entre os fatores dois a dois sejam proporcionais".

Para a prova do teorema 2 utilizou-se a mesma seqüência apresentada na prova do teorema 1, mas agora aplicada aos fatores dois a dois. Na seqüência discutem-se, detalhadamente, as formas de obtenção das proporções para as freqüências de caselas de um modelo com três fatores.

\section{a) Ortogonalidade entre os subespaços gerados pelas colunas das submatrizes de $X$ referentes aos fatores $A$ e $B$}

Se num modelo com três fatores, conforme descrito na definição 2, o subespaço gerado pelas colunas de $X_{\alpha \mid \mu}$ é ortogonal ao subespaço gerado pelas colunas de $X_{\beta \mid \mu}$, então:

$$
C\left(X_{\alpha \mid \mu}\right) \perp C\left(X_{\beta \mid \mu}\right)
$$

Da aplicação do teorema 2 para os fatores $A$ e $B$, obteve-se a seguinte condição:

$$
C\left(X_{\alpha \mid \mu}\right) \perp C\left(X_{\beta \mid \mu}\right) \Leftrightarrow \frac{n_{i j .}}{n_{i j^{\prime} .}}=\frac{n_{i^{\prime} j .}}{n_{i j^{\prime} j^{\prime}}}, \quad \forall\left(i \neq i^{\prime} ; j \neq j^{\prime}\right)
$$

A prova do teorema 2, como dito anteriormente, é uma extensão da prova do teorema 1 . Algumas diferenças na notação diferenciam a maneira de provar de cada uma. Assim, os passos em comum não foram apresentados. $\mathrm{Na}$ seqüência são apresentadas apenas as etapas da prova do teorema 2 ainda não detalhadas neste texto. 
Da condição de ortogonalidade apresentada em (2), tem-se:

$$
C\left(X_{\alpha \mid \mu}\right) \perp C\left(X_{\beta \mid \mu}\right) \Leftrightarrow P_{\mu, \alpha} P_{\mu, \beta}=P_{\mu}
$$

O projetor referente a $\mu$ é dado por:

$$
P_{\mu}=\left(p_{i j k l}\right) ; \quad p_{i j k l}=\frac{1}{n_{\ldots}}-\forall(i, j, k, l)
$$

Da expressão 3, tem-se:

$$
P_{\mu, \alpha} P_{\mu, \beta}=X_{\alpha}\left(X_{\alpha}^{\prime} X_{\alpha}\right)^{-1} X_{\alpha}^{\prime} X_{\beta}\left(X_{\beta}^{\prime} X_{\beta}\right)^{-1} X_{\beta}^{\prime}
$$

onde para um modelo com três fatores, os termos são:

$$
\left(X_{\alpha}^{\prime} X_{\alpha}\right)^{-1}=\operatorname{diag}\left\{1 / n_{i . .}\right\} ; \quad\left(X_{\beta}^{\prime} X_{\beta}\right)^{-1}=\operatorname{diag}\left\{1 / n_{. j .}\right\} ; \quad X_{\alpha}^{\prime} X_{\beta}=\left(x_{i j .}\right), x_{i j .}=n_{i j} .
$$

O produto matricial da expressão 8 , é apresentado na seqüência:

$$
P_{\mu, \alpha} P_{\mu, \beta}=X_{\alpha}\left[\begin{array}{cc}
1 / n_{i . .} & 0 \\
0 & 1 / n_{i^{\prime} . .}
\end{array}\right]\left[\begin{array}{ll}
n_{i j .} & n_{i j^{\prime} .} \\
n_{i^{\prime} j .} & n_{i^{\prime} j^{\prime}}
\end{array}\right]\left[\begin{array}{cc}
1 / n_{. j .} & 0 \\
0 & 1 / n_{. j^{\prime} .}
\end{array}\right] X_{\beta}^{\prime}
$$

Assim, pode-se representar cada termo da expressão 8 por:

$$
P_{\mu, \alpha} P_{\mu, \beta}=\left(p_{i j . l}^{*}\right), \quad p_{i j . l}^{*}=\frac{n_{i j .}}{n_{i . .} n_{. j .}}
$$

Portanto, da condição de ortogonalidade dada em (2), tem-se:

$$
\frac{n_{i j .}}{n_{i . .} n_{. j .}}=\frac{1}{n_{\ldots}}
$$

Desse modo,

$$
C\left(X_{\alpha \mid \mu}\right) \perp C\left(X_{\beta \mid \mu}\right) \Leftrightarrow \frac{n_{i j .}}{n_{i . .} n_{. j .}}=\frac{1}{n_{\ldots}}
$$


obtendo-se a seguinte proporção entre as freqüências de caselas,

$$
\frac{n_{i j .}}{n_{i j^{\prime} .}}=\frac{n_{i^{\prime} j .}}{n_{i^{\prime} j^{\prime} .}}, \quad \forall(i \neq i ; j \neq j)
$$

\section{b) Ortogonalidade entre os subespaços gerados pelas colunas das submatrizes de $X$ referentes aos fatores $A$ e $C$}

Se num modelo com três fatores, conforme descrito na definição 2, o subespaço gerado pelas colunas de $X_{\alpha \mid \mu}$ é ortogonal ao subespaço gerado pelas colunas de $X_{\text {ylu }}$, então:

$$
C\left(X_{\alpha \mid \mu}\right) \perp C\left(X_{\gamma \mid \mu}\right)
$$

Assim como para os fatores $A$ e $B$, prova-se o teorema 2 para os fatores $A$ e $C$, aplicando-se as mesmas deduçōes feitas para o teorema 1.

$$
C\left(X_{\alpha \mid \mu}\right) \perp C\left(X_{\gamma \mid \mu}\right) \Leftrightarrow \frac{n_{i . k}}{n_{i . k^{\prime}}}=\frac{n_{i^{\prime} . k}}{n_{i^{\prime} . k^{\prime}}} \quad \forall\left(i \neq i^{\prime} ; j \neq j^{\prime}\right)
$$

Adaptando-se a condição de ortogonalidade dada em (2), para os fatores $A$ e $C$, tem-se:

$$
C\left(X_{\alpha \mid \mu}\right) \perp C\left(X_{\gamma \mid \mu}\right) \Leftrightarrow P_{\alpha \mid \mu} P_{\gamma \mid \mu}=P_{\mu}
$$

O projetor referente a $\mu$, como já mencionado, é:

$$
P_{\mu}=\left(p_{i j k l}\right) ; \quad p_{i j k l}=\frac{1}{n_{\ldots}}, \quad \forall(i, j, k, l)
$$


Adaptando-se a expressão 3, para os fatores $A$ e $C$, obtém-se:

$$
P_{\mu, \alpha} P_{\mu, \gamma}=X_{\alpha}\left(X_{\alpha}^{\prime} X_{\alpha}\right)^{-1} X_{\alpha}^{\prime} X_{\gamma}\left(X_{\gamma}^{\prime} X_{\gamma}\right)^{-1} X_{\gamma}^{\prime}
$$

onde:

$$
\left(X_{\alpha}^{\prime} X_{\alpha}\right)^{-1}=\operatorname{diag}\left\{1 / n_{i . .}\right\} ; \quad\left(X_{\gamma}^{\prime} X_{\gamma}\right)^{-1}=\operatorname{diag}\left\{1 / n_{. . k}\right\} ; \quad X_{\alpha}^{\prime} X_{\gamma}=\left(x_{i . k}\right), x_{i . k}=n_{i . k}
$$

O produto matricial da expressão 3 , é apresentado na seqüência.

$$
P_{\mu, \alpha} P_{\mu, \gamma}=X_{\alpha}\left[\begin{array}{cc}
1 / n_{i . .} & 0 \\
0 & 1 / n_{i . .}
\end{array}\right]\left[\begin{array}{ll}
n_{i . k} & n_{i . k^{\prime}} \\
n_{i^{\prime} . k} & n_{i^{\prime} . k^{\prime}}
\end{array}\right]\left[\begin{array}{cc}
1 / n_{. . k} & 0 \\
0 & 1 / n_{. . k^{\prime}}
\end{array}\right] X_{\gamma}^{\prime}
$$

Assim, pode-se representar cada termo da expressão 3 por:

$$
P_{\mu, \alpha} P_{\mu, \gamma}=\left(p_{i . k l}^{*}\right), \quad p_{i . k l}^{*}=\frac{n_{i . k}}{n_{i . .} n_{. . k}}
$$

Portanto, da condição de ortogonalidade dada na expressão 12, obteve-se:

$$
\frac{n_{i . k}}{n_{i . .} n_{. k}}=\frac{1}{n_{\ldots}}
$$

Desse modo,

$$
C\left(X_{\alpha \mid \mu}\right) \perp C\left(X_{\gamma \mid \mu}\right) \Leftrightarrow \frac{n_{i . k}}{n_{i . .} n_{. . k}}=\frac{1}{n_{\ldots}}
$$

obtendo-se a seguinte proporção entre as freqüências de caselas,

$$
\frac{n_{i . k}}{n_{i . k^{\prime}}}=\frac{n_{i^{\prime} . k}}{n_{i^{\prime} . k^{\prime}}} \quad \forall(i \neq i, k \neq k)
$$


c) Ortogonalidade entre os subespaços gerados pelas colunas das submatrizes de $X$ referentes aos fatores $B$ e $C$

Se num modelo com três fatores, conforme descrito na definição 2 , o subespaço gerado pelas colunas de $X_{\beta \mid \mu}$ é ortogonal ao subespaço gerado pelas colunas de $X_{\gamma \mid \mu}$, então:

$$
C\left(X_{\beta \mid \mu}\right) \perp C\left(X_{\gamma \mid \mu}\right)
$$

Para os fatores $B$ e $C$, prova-se que:

$$
C\left(X_{\beta \mid \mu}\right) \perp C\left(X_{\gamma \mid \mu}\right) \Leftrightarrow \frac{n_{\cdot j k}}{n_{\cdot j k^{\prime}}}=\frac{n_{\cdot j^{\prime} k}}{n_{\cdot j^{\prime} k^{\prime}}} \quad \forall\left(j \neq j^{\prime} ; k \neq k^{\prime}\right)
$$

A condição de ortogonalidade dada em (2), adaptada para os fatores $B$ e $C$, é:

$$
C\left(X_{\beta \mid \mu}\right) \perp C\left(X_{\gamma \mid \mu}\right) \Leftrightarrow P_{\mu, \beta} P_{\mu, \gamma}=P_{\mu}
$$

onde:

$$
P_{\mu}=\left(p_{i j k l}\right) ; \quad p_{i j k l}=\frac{1}{n_{\ldots}}, \quad \forall(i, j, k, l)
$$

Da expressão 3, adaptada para os fatores $B$ e $C$, obtém-se:

$$
P_{\mu, \beta} P_{\mu, \gamma}=X_{\beta}\left(X_{\beta}^{\prime} X_{\beta}\right)^{1} X_{\beta}^{\prime} X_{\gamma}\left(X_{\gamma}^{\prime} X_{\gamma}\right)^{-1} X_{\gamma}^{\prime}
$$

onde:

$\left(X_{\beta}^{\prime} X_{\beta}\right)^{-1}=\operatorname{diag}\left\{1 / n_{. j .}\right\} ; \quad\left(X_{\gamma}^{\prime} X_{\gamma}\right)^{-1}=\operatorname{diag}\left\{1 / n_{. k}\right\} ; \quad X_{\beta}^{\prime} X_{\gamma}=\left(x_{. j k}\right), x_{. j k}=n_{. j k}$

O produto matricial da expressão 18, é apresentado na seqüência.

$$
P_{\mu, \beta} P_{\mu, \gamma}=X_{\beta}\left[\begin{array}{cc}
1 / n_{. j .} & 0 \\
0 & 1 / n_{. j^{\prime}}
\end{array}\right]\left[\begin{array}{cc}
n_{. j k} & n_{\cdot j k^{\prime}} \\
n_{. j^{\prime} k} & n_{j^{\prime} k^{\prime}}
\end{array}\right]\left[\begin{array}{cc}
1 / n_{. . k} & 0 \\
0 & 1 / n_{. k^{\prime}}
\end{array}\right] X_{\gamma}^{\prime}
$$


Representando-se cada termo da expressão 18, obtém-se:

$$
P_{\mu, \beta} P_{\mu, \gamma}=\left(p_{i . k l}^{*}\right), \quad p_{i . k l}^{*}=\frac{n_{. j k}}{n_{. j .} \cdot n_{. k k}}
$$

Portanto, da condição de ortogonalidade dada em (17), tem-se a seguinte igualdade,

$$
\frac{n_{. j k}}{n_{. j .} n_{. . k}}=\frac{1}{n_{\ldots}}
$$

Desse modo,

$$
C\left(X_{\beta \mid \mu}\right) \perp C\left(X_{\gamma \mid \mu}\right) \Leftrightarrow \frac{n_{. j k}}{n_{. j .} n_{. . k}}=\frac{1}{n_{. . .}}
$$

obtendo-se na seguinte proporção entre as freqüências de caselas:

$$
\frac{n_{\cdot j k}}{n_{. j k^{\prime}}}=\frac{n_{\cdot j^{\prime} k}}{n_{\cdot j^{\prime} k^{\prime}}}, \quad \forall\left(j \neq j^{\prime}, k \neq k\right)
$$

\subsubsection{Modelos com quatro fatores}

Definiçẫo 3. Um delineamento com quatro fatores cruzados sem interações denotado por: $y_{i j k l m}=\mu+\alpha_{i}+\beta_{j}+\gamma_{k}+\varphi_{l}+e_{i j k l m}$ com $i=1, \ldots, a$ niveis do fator $A ; j=1, \ldots, b$ niveis do fator $B ; k=1, \ldots, c$ niveis do fator $C$; $l=1, \ldots, d$ niveis do fator $D$ e $m=1, \ldots, n_{i j k l}$ observações na casela $(i ; j ; k ; l)$ é dito ortogonal, se o subespaço gerado pelas colunas da submatriz de $X$ referentes a cada fator, após o ajuste de $\mu$, é individualmente ortogonal aos subespaços gerados pelas colunas das submatrizes de $X$ referentes aos outros três fatores, após o ajuste de $\mu$. 
A matriz do modelo linear $y=X \theta+e$ apresenta, então, a seguinte partição:

$$
X=\left[\begin{array}{l:l:l:l:l}
X_{\mu} & X_{\alpha} & X_{\beta} & X_{\gamma} & X_{\varphi}
\end{array}\right]
$$

onde:

$X_{\mu}$ é a submatriz de $X$ referente a constante $\mu$;

$X_{\alpha}$ é a submatriz de $X$ referente ao fator $A$;

$X_{\beta}$ é a submatriz de $X$ referente ao fator $B$;

$X_{\gamma}$ é a submatriz de $X$ referente ao fator $C$;

$X_{\varphi}$ é a submatriz de $X$ referente ao fator $D$.

obtendo-se $X_{\alpha \mid \mu}, X_{\beta \mid \mu}, X_{\gamma \mid \mu}$ e $X_{\varphi \mid \mu}$ como na definição 2 .

Para provar a ortogonalidade através da proporcionalidade de freqüências de caselas dos fatores dois a dois, utilizou-se uma adaptação do teorema 2, da mesma forma aplicada nos modelos com três fatores, obtendo-se os seguintes resultados.

a) Ortogonalidade entre os subespaços gerados pelas colunas das submatrizes de $X$ referentes aos fatores $A$ e $B$

$$
C\left(X_{\alpha \mid \mu}\right) \perp C\left(X_{\beta \mid \mu}\right) \Leftrightarrow \frac{n_{i j . .}}{n_{i j^{\prime} .}}=\frac{n_{i^{\prime} j . .}}{n_{i^{\prime} j^{\prime} .}}
$$

b) Ortogonalidade entre os subespaços gerados pelas colunas das submatrizes de $X$ referentes aos fatores $A$ e $C$

$$
C\left(X_{\alpha \mid \mu}\right) \perp C\left(X_{\gamma \mid \mu}\right) \Leftrightarrow \frac{n_{i . k .}}{n_{i . k^{\prime} .}}=\frac{n_{i^{\prime} . k}}{n_{i^{\prime} . k^{\prime}}}
$$


c) Ortogonalidade entre os subespaços gerados pelas colunas das submatrizes de $X$ referentes aos fatores $A$ e $D$

$$
C\left(X_{\alpha \mid \mu}\right) \perp C\left(X_{\varphi \mid \mu}\right) \Leftrightarrow \frac{n_{i . l l}}{n_{i . . l^{\prime}}}=\frac{n_{i^{\prime} . . l}}{n_{i^{\prime} . . l^{\prime}}}
$$

d) Ortogonalidade entre os subespaços gerados pelas colunas das submatrizes de $X$ referentes aos fatores $B$ e $C$

$$
C\left(X_{\beta \mid \mu}\right) \perp C\left(X_{\gamma \mid \mu}\right) \Leftrightarrow \frac{n_{\cdot j k .}}{n_{\cdot j k^{\prime} .}}=\frac{n_{\cdot j^{\prime} k .}}{n_{\cdot j^{\prime} k^{\prime}}}
$$

e) Ortogonalidade entre os subespaços gerados pelas colunas das submatrizes de $X$ referentes aos fatores $B$ e $D$

$$
C\left(X_{\beta \mid \mu}\right) \perp C\left(X_{\varphi \mid \mu}\right) \Leftrightarrow \frac{n_{j . l}}{n_{\cdot j \cdot l^{\prime}}}=\frac{n_{\cdot j^{\prime} . l}}{n_{\cdot j^{\prime} \cdot l^{\prime}}}
$$

f) Ortogonalidade entre os subespaços gerados pelas colunas das submatrizes de $X$ referentes aos fatores $C$ e $D$

$$
C\left(X_{\gamma \mid \mu}\right) \perp C\left(X_{\varphi \mid \mu}\right) \Leftrightarrow \frac{n_{. . k l}}{n_{. . k l^{\prime}}}=\frac{n_{. . k^{\prime} l}}{n_{. . k^{\prime} l^{\prime}}}
$$

\subsubsection{Modelos com $K$ fatores}

Definição 4. Um delineamento com $K$ fatores é dito ortogonal, se o subespaço gerado pelas colunas da submatriz de $X$ referentes a cada fator, após o ajuste de $\mu$, é individualmente ortogonal aos subespaços gerados pelas colunas das submatrizes de $X$ referentes aos outros $K$-1 fatores, após o ajuste de $\mu$. 


\subsubsection{Propriedades dos delineamentos ortogonais}

Dados os objetivos deste estudo, as propriedades aqui descritas estão direcionadas para as funções estimáveis. Ademais, embora elas independam do sistema computacional estatístico utilizado, a abordagem é feita à luz do Proc GLM do sistema estatistico SAS.

Sua descrição tem, aqui, o objetivo de estender sua validade para modelos com mais de dois fatores. Sendo assim seguem, entre parênteses, os nomes de autores que discutiram sua validade nos modelos com dois fatores.

\subsection{Quanto às funções estimáveis}

\section{a) Modelo sem interações}

P.1. As funções estimáveis de um fator contêm apenas os parâmetros relativos ao próprio fator e não contêm os parâmetros relativos aos outros fatores.

P.2 As funções estimáveis do tipo I são equivalentes às funções estimáveis do tipo II, uma vez que sob ortogonalidade (Iemma \& Claustriaux, 1997), as médias ponderadas são iguais às médias ponderadas ajustadas.

P.3 Dado que o termo completo é condição necessária para a ortogonalidade (Iemma \& Claustriaux, 1997), as funções estimáveis dos tipos III e IV são equivalentes.

P.4 Como o modelo não contém interações, as funções estimáveis dos tipos II e III são equivalentes (Speed, Hocking \& Hackney, 1978).

P.5 Decorre das propriedades anteriores que, se o modelo é ortogonal e não contém interações, os quatro tipos de funções estimáveis são equivalentes, quer seja ou não balanceado. 


\section{b) Modelo com interações}

\section{b.1) Efeitos principais}

Se o delineamento ortogonal é desbalanceado, as funções estimáveis preservam as propriedades P.2 e P.3, às quais acrescentam-se:

P.6 As funções estimáveis de um fator contêm os parâmetros relativos ao próprio fator e os respectivos parâmetros das interações onde o fator está presente e não contêm os parâmetros relativos aos outros fatores, nem mesmo os parâmetros das interaçōes nas quais o fator não está presente.

Se o delineamento ortogonal é balanceado, acrescenta-se ainda a propriedade:

P.7 Num delineamento ortogonal balanceado, os quatro tipos de funções estimáveis são equivalentes, quer o modelo contenha ou não interações.

\section{b.2) Interações}

P.8. As funções estimáveis de uma interação entre fatores, contêm os parâmetros relativos à própria interação e os parâmetros das interações de grau maior que o grau da interação em estudo e não contêm os parâmetros relativos aos efeitos principais, nem mesmo os parâmetros das interações de grau menor que o grau da interação em estudo.

\subsection{Quanto às hipóteses}

As propriedades descritas em 3.2.2.5.1 para as funções estimáveis, são mantidas para as hipóteses correspondentes.

Dado que as propriedades P.1 e P.2 são intrínsecas dos delineamentos ortogonais, apenas as hipóteses dos tipos I e II, isto é, apenas as hipóteses sobre médias ponderadas e sobre médias ponderadas ajustadas, serão discutidas neste item. O Quadro 4 descreve as somas de quadrados, 
através da notação $R($.$) , conforme Searle (1971), para as hipóteses dos tipos I e$ II em modelos com até quatro fatores ortogonais. Para os modelos com mais de quatro fatores, poderão ser feitas extensōes da Quadro 4 com adaptaçōes em função da notação utilizada. 


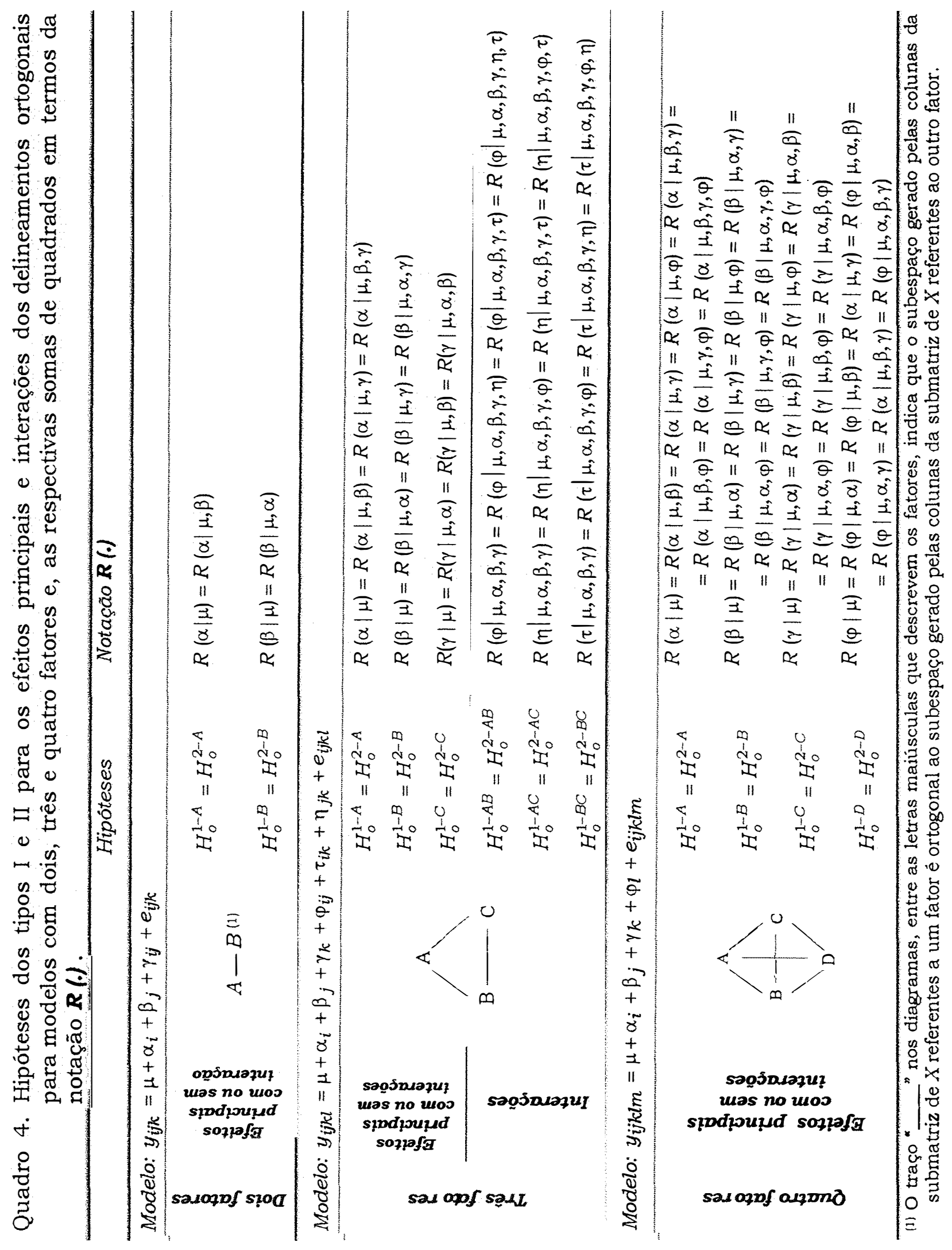




\subsubsection{Delineamentos parcialmente ortogonais}

Delineamentos parcialmente ortogonais são aqueles nos quais, entre todas combinaçōes entre fatores dois a dois, apenas algumas apresentam freqüências de caselas proporcionais, ou seja, para alguns fatores do modelo o subespaço gerado pelas colunas da submatriz de $X$ referentes a esses fatores, após o ajuste de $\mu$, é individualmente ortogonal ao subespaço gerado pelas colunas da submatriz de X referentes aos demais fatores, após o ajuste de $\mu$.

Nos modelos com dois fatores, não faz sentido definir delineamento parcialmente ortogonal, uma vez que entre dois fatores as freqüências das caselas são ou não são proporcionais, definindo o delineamento como ortogonal ou não ortogonal, respectivamente.

A grande importância dos delineamentos parcialmente ortogonais consiste na possibilidade que tem o pesquisador, de planejar o experimento de tal modo que a ortogonalidade seja garantida para o fator ou para os fatores de maior interesse. Além disso, quando o número de fatores no modelo aumenta é mais dificil e oneroso estabelecer freqüências de caselas constantes ou mesmo proporcionais para todas as combinaçōes.

\subsubsection{Modelos com três fatores}

Definiçâo 5. Um delineamento com três fatores cruzados sem interaçōes caracterizado por $y_{i j k l}=\mu+\alpha_{i}+\beta_{j}+\gamma_{k}+e_{i j k l}$ com $i=1, \ldots, a$ niveis do fator $A ; j=1, \ldots, b$ niveis do fator $B ; k=1, \ldots, c$ niveis do fator $C$ e $l=1, \ldots, n_{i j k}$ observaçōes na casela $(i ; j ; k)$ é dito parcialmente ortogonal, se o subespaço gerado pelas colunas da submatriz de $X$ referentes a apenas um dos fatores, após o ajuste de $\mu$, é individualmente ortogonal aos subespaços gerados pelas colunas das submatrizes de $X$ referentes aos outros dois fatores, após o ajuste de $\mu$. 
A matriz do modelo linear $y=X \theta+e$ apresenta, então, a seguinte partição:

$$
X=\left[\begin{array}{l:l:l:l}
X_{\mu} & X_{\alpha} & X_{\beta} & X \gamma
\end{array}\right]
$$

onde:

$X_{\mu}$ é a submatriz de $X$ referente a constante $\mu$;

$X_{\alpha}$ é a submatriz de $X$ referente ao fator $A$;

$X_{\beta}$ é a submatriz de $X$ referente ao fator $B$;

$X_{\gamma}$ é a submatriz de $X$ referente ao fator $C$.

E, de modo análogo à definição 1 obtém-se: $X_{\alpha \mid \mu}, X_{\beta \mid \mu}$ e $X_{\gamma \mid \mu}$.

Num delineamento parcialmente ortogonal com três fatores, para o modelo descrito na definição 5 , três casos distintos podem ocorrer:

Caso 1) Apenas o subespaço gerado pelas colunas de $X_{\alpha \mid \mu}$ é individualmente ortogonal aos subespaços gerados pelas colunas de $X_{\beta \mid \mu}$ e $X_{\gamma \mid \mu}$.

Em termos de freqüências de caselas, tem-se:

$$
C\left(X_{\alpha \mid \mu}\right) \perp C\left(X_{\beta \mid \mu}\right) \Leftrightarrow \frac{n_{i j .}}{n_{i j^{\prime} .}}=\frac{n_{i^{\prime} j .}}{n_{i^{\prime} j^{\prime} .}} ; \quad C\left(X_{\alpha \mid \mu}\right) \perp C\left(X_{\gamma \mid \mu}\right) \Leftrightarrow \frac{n_{i . k}}{n_{i . k^{\prime}}}=\frac{n_{i^{\prime} . k}}{n_{i^{\prime} . k^{\prime}}}
$$

Caso 2) Apenas o subespaço gerado pelas colunas de $X_{\beta \mid \mu}$ é individualmente ortogonal aos subespaços gerados pelas colunas de $X_{\alpha \mid \mu}$ e $X_{\gamma \mid \mu}$.

Em termos de freqüências de caselas, tem-se:

$$
C\left(X_{\beta \mid \mu}\right) \perp C\left(X_{\alpha \mid \mu}\right) \Leftrightarrow \frac{n_{i j .}}{n_{i j^{\prime} .}}=\frac{n_{i^{\prime} j .}}{n_{i^{\prime} j^{\prime} .}} ; \quad C\left(X_{\beta \mid \mu}\right) \perp C\left(X_{\gamma \mid \mu}\right) \Leftrightarrow \frac{n_{\cdot j k}}{n_{\cdot j k^{\prime}}}=\frac{n_{\cdot j^{\prime} k}}{n_{\cdot j^{\prime} k^{\prime}}}
$$


Caso 3) Apenas o subespaço gerado pelas colunas de $X_{\gamma \mid \mu}$ é individualmente ortogonal aos subespaços gerados pelas colunas de $X_{\alpha \mid \mu}$ e $X_{\beta \mid \mu}$.

Em termos de freqüências de caselas, tem-se:

$$
C\left(X_{\gamma \mid \mu}\right) \perp C\left(X_{\alpha \mid \mu}\right) \Leftrightarrow \frac{n_{i . k}}{n_{i \cdot k^{\prime}}}=\frac{n_{i^{\prime} \cdot k}}{n_{i^{\prime} \cdot k^{\prime}}} ; C\left(X_{\gamma \mid \mu}\right) \perp C\left(X_{\beta \mid \mu}\right) \Leftrightarrow \frac{n_{\cdot j k}}{n_{\cdot j k^{\prime}}}=\frac{n_{\cdot j k}}{n_{\cdot j^{\prime} k^{\prime}}}
$$

\subsubsection{Modelos com quatro fatores}

Definição 6. Um delineamento com quatro fatores cruzados sem interações caracterizado por: $y_{i j k l m}=\mu+\alpha_{i}+\beta_{j}+\gamma_{k}+\varphi_{l}+e_{i j k l m}$ com $i=1, \ldots, a$ niveis do fator $A ; j=1, \ldots, b$ niveis do fator $B ; k=1, \ldots, c$ niveis do fator $C ; l=1, \ldots$, $d$ niveis do fator $D$ e $m=1, \ldots, n_{i j k l}$ observações na casela $(i ; j ; k ; l)$, é dito parcialmente ortogonal, se os subespaços gerados pelas colunas das submatrizes de $X$ referentes, no máximo, à dois fatores, após o ajuste de $\mu$, são individualmente ortogonais aos subespaços gerados pelas colunas das submatrizes de $X$ referentes os outros três fatores, após o ajuste de $\mu$.

A matriz do modelo linear $y=X \theta+e$ apresenta, então, a seguinte partição:

$$
X=\left[\begin{array}{l:l:l:l:l}
X_{\mu} & X_{\alpha} & X_{\beta} & X_{\gamma} & X_{\varphi}
\end{array}\right]
$$

onde:

$X_{\mu}$ é a submatriz de $X$ referente a constante $\mu$;

$X_{\alpha}$ é a submatriz de $X$ referente ao fator $A$;

$X_{\beta}$ é a submatriz de $X$ referente ao fator $B$;

$X_{\gamma}$ é a submatriz de $X$ referente ao fator $C$;

$X_{\varphi}$ é a submatriz de $X$ referente ao fator $D$.

obtendo-se $X_{\alpha \mid \mu}, X_{\beta \mid \mu}, X_{\gamma \mid \mu}$ e $X_{\varphi \mid \mu}$ como na definição 2 .

Nos delineamentos parcialmente ortogonais com quatro fatores, para o modelo descrito na definição 6, podem ocorrer duas situações distintas. 
A primeira situaçāo foi subdividida em quatro casos e a segunda situaçāo em seis casos distintos.

Situação 1) Apenas o subespaço gerado pelas colunas da submatriz de $X$ referentes a um dos fatores, após o ajuste de $\mu$, é individualmente ortogonal aos subespaços gerados pelas colunas das submatrizes de $X$ referentes aos outros três fatores, após o ajuste de $\mu$. Entretanto, se outras combinações entre fatores apresentarem subespaços ortogonais, de tal modo que apenas um dos fatores continue ortogonal aos demais, os casos descritos na seqüência ainda serāo válidos. Nessa situaçāo, quatro casos distintos podem ocorrer:

Caso 1) Quando o subespaço do fator $A$ for ortogonal aos demais

$$
\begin{gathered}
C\left(X_{\left.\alpha\right|_{\mu}}\right) \perp C\left(X_{\beta(\mu}\right), C\left(X_{\alpha \mid \mu}\right) \perp C\left(X_{\gamma \mid \mu}\right) \text { e } C\left(X_{\left.\alpha\right|_{\mu}}\right) \perp C\left(X_{\varphi \mid \mu}\right) \text {, então: } \\
\frac{n_{i j .}}{n_{i j^{\prime} .}}=\frac{n_{i^{\prime} j .}}{n_{i^{\prime} j^{\prime} .}} \text { e } \frac{n_{i . k}}{n_{i . k^{\prime}}}=\frac{n_{i^{\prime} . k}}{n_{i^{\prime} . k^{\prime}}} \text { e } \frac{n_{i . . l}}{n_{i . . l^{\prime}}}=\frac{n_{i^{\prime} . . l}}{n_{i^{\prime} . . l^{\prime}}}
\end{gathered}
$$

Caso 2) Quando o subespaço do fator $B$ for ortogonal aos demais

$$
\begin{gathered}
C\left(X_{\beta \mid \mu}\right) \perp C\left(X_{\alpha \mid \mu}\right), C\left(X_{\beta \mid \mu}\right) \perp C\left(X_{\gamma \mid \mu}\right) \text { e } C\left(X_{\beta \mid \mu}\right) \perp C\left(X_{\phi \mid \mu}\right) \text {, então: } \\
\frac{n_{i j .}}{n_{i j^{\prime} .}}=\frac{n_{i^{\prime} j .}}{n_{i^{\prime} j^{\prime} .}} \text { e } \frac{n_{\cdot j k}}{n_{\cdot j k^{\prime}}}=\frac{n_{\cdot j^{\prime} k}}{n_{\cdot j^{\prime} k^{\prime}}} \text { e } \frac{n \cdot \cdot j \cdot l}{n_{\cdot j \cdot l^{\prime}}}=\frac{n_{\cdot j^{\prime} \cdot l}}{n_{\cdot j^{\prime} \cdot l^{\prime}}}
\end{gathered}
$$

Caso 3) Quando o subespaço do fator $C$ for ortogonal aos demais

$$
\begin{gathered}
C\left(X_{\gamma \mid \mu}\right) \perp C\left(X_{\alpha \mid \mu}\right), C\left(X_{\gamma \mid \mu}\right) \perp C\left(X_{\beta \mid \mu}\right) \text { e } C\left(X_{\gamma \mid \mu}\right) \perp C\left(X_{\varphi \mid \mu}\right) \text {, então: } \\
\frac{n_{i . k}}{n_{i . k^{\prime}}}=\frac{n_{i^{\prime} . k}}{n_{i^{\prime} . k^{\prime}}} \text { e } \frac{n_{. j k}}{n_{. j k^{\prime}}}=\frac{n_{. j^{\prime} k}}{n_{. j^{\prime} k^{\prime}}} \text { e } \frac{n_{. . k l}}{n_{. . k l^{\prime}}}=\frac{n_{. . k^{\prime} l}}{n_{. . k l^{\prime}}}
\end{gathered}
$$


Caso 4) Quando o subespaço do fator $D$ for ortogonal aos demais

$$
\begin{gathered}
C\left(X_{\phi \phi_{\mu}}\right) \perp C\left(X_{\left.\alpha\right|_{\mu}}\right), C\left(X_{\left.\phi\right|_{\mu}}\right) \perp C\left(X_{\left.\beta\right|_{\mu}}\right) \text { e } C\left(X_{\left.\varphi\right|_{\mu}}\right) \perp C\left(X_{\left.\gamma\right|_{\mu}}\right) \text {, então: } \\
\frac{n_{i . . l}}{n_{i . l^{\prime}}}=\frac{n_{i^{\prime} . l}}{n_{i^{\prime} . l^{\prime}}} \text { e } \frac{n_{\cdot . j . l}}{n_{. j . l^{\prime}}}=\frac{n_{. j^{\prime} . l}}{n_{. j^{\prime} . l^{\prime}}} \text { e } \frac{n_{. . k l}}{n_{. . k l^{\prime}}}=\frac{n_{. . k^{\prime} l}}{n_{. . k^{\prime} l^{\prime}}}
\end{gathered}
$$

Situação 2) O subespaço gerado pelas colunas da submatriz de $X$ referentes a dois fatores, após o ajuste de $\mu$, é individualmente ortogonal aos subespaços gerados pelas colunas das submatrizes de $X$ referentes aos outros dois fatores, após o ajuste de $\mu$. Assim, seis casos distintos podem ocorrer:

Caso 1) Quando os subespaços de $A$ e $B$ forem ortogonais aos demais

$$
\begin{aligned}
& C\left(X_{\left.\alpha\right|_{\mu}}\right) \perp C\left(X_{\left.\beta\right|_{\mu}}\right), C\left(X_{\left.\alpha\right|_{\mu}}\right) \perp C\left(X_{\gamma \mid \mu}\right), C\left(X_{\left.\alpha\right|_{\mu}}\right) \perp C\left(X_{\left.\varphi\right|_{\mu}}\right), C\left(X_{\left.\beta\right|_{\mu}}\right) \perp C\left(X_{\gamma \mid \mu}\right), \\
& C\left(X_{\left.\beta\right|_{\mu}}\right) \perp C\left(X_{\left.\varphi\right|_{\mu}}\right), \text { então: } \\
& \quad \frac{n_{i j .}}{n_{i j^{\prime} .}}=\frac{n_{i^{\prime} j .}}{n_{i^{\prime} j^{\prime} \cdot}} \text { e } \frac{n_{i . k}}{n_{i \cdot k^{\prime}}}=\frac{n_{i^{\prime} \cdot k}}{n_{i^{\prime} \cdot k^{\prime}}} \text { e } \frac{n_{i . . l}}{n_{i . l^{\prime}}}=\frac{n_{i^{\prime} . l}}{n_{i^{\prime} . l^{\prime}}} \text { e } \frac{n_{\cdot j k}}{n_{\cdot j k^{\prime}}}=\frac{n_{\cdot j^{\prime} k}}{n_{\cdot j^{\prime} k^{\prime}}} \text { e } \frac{n_{\cdot j \cdot j}}{n_{\cdot j \cdot l^{\prime}}}=\frac{n_{\cdot j^{\prime} \cdot l}}{n_{\cdot j^{\prime} \cdot l^{\prime}}}
\end{aligned}
$$

Caso 2) Quando os subespaços de $A$ e $C$ forem ortogonais aos demais

$$
\begin{aligned}
& C\left(X_{\left.\alpha\right|_{\mu}}\right) \perp C\left(X_{\beta \mid \mu}\right), C\left(X_{\left.\alpha\right|_{\mu}}\right) \perp C\left(X_{\gamma \mid \mu}\right), C\left(X_{\left.\alpha\right|_{\mu}}\right) \perp C\left(X_{\varphi \mid \mu}\right), C\left(X_{\gamma \mid \mu}\right) \perp C\left(X_{\beta \mid \mu}\right), \\
& C\left(X_{\gamma \mid \mu}\right) \perp C\left(X_{\varphi \mid \mu}\right), \text { então: } \\
& \quad \frac{n_{i j .}}{n_{i j^{\prime} .}}=\frac{n_{i^{\prime} j .}}{n_{i^{\prime} j^{\prime} .}} \text { e } \frac{n_{i . k}}{n_{i . k^{\prime}}}=\frac{n_{i^{\prime} . k}}{n_{i^{\prime} \cdot k^{\prime}}} \text { e } \frac{n_{i . l}}{n_{i . . l^{\prime}}}=\frac{n_{i^{\prime} . l l}}{n_{i^{\prime} . . l^{\prime}}} \text { e } \frac{n_{. j k}}{n_{. j k^{\prime}}}=\frac{n_{. j^{\prime} k}}{n_{. j^{\prime} k^{\prime}}} \text { e } \frac{n_{. k l}}{n_{. . k l^{\prime}}}=\frac{n_{. k^{\prime} l}}{n_{. . k l^{\prime}}}
\end{aligned}
$$


Caso 3) Quando os subespaços de $A$ e $D$ forem ortogonais aos demais $C\left(X_{\alpha \mid \mu}\right) \perp C\left(X_{\beta \mid \mu}\right), C\left(X_{\left.\alpha\right|_{\mu}}\right) \perp C\left(X_{\gamma \mid \mu}\right), C\left(X_{\left.\alpha\right|_{\mu}}\right) \perp C C\left(X_{\varphi \mid \mu}\right), C\left(X_{\phi \mid \mu}\right) \perp C\left(X_{\beta \mid \mu}\right)$, $C\left(X_{\varphi \mid \mu}\right) \perp C\left(X_{\gamma \mid \mu}\right)$, então:

$$
\frac{n_{i j .}}{n_{i j^{\prime} .}}=\frac{n_{i^{\prime} . j}}{n_{i^{\prime} j^{\prime} .}} \text { e } \frac{n_{i . k}}{n_{i . k^{\prime}}}=\frac{n_{i^{\prime} . k}}{n_{i^{\prime} . k^{\prime}}} \text { e } \frac{n_{i . . l}}{n_{i . . l^{\prime}}}=\frac{n_{i^{\prime} . l}}{n_{i^{\prime} . . l^{\prime}}} \text { e } \frac{n_{. j . j . l}}{n_{. j . l^{\prime}}}=\frac{n_{. j^{\prime} . l}}{n_{. j^{\prime} . l^{\prime}}} \text { e } \frac{n_{. . k l}}{n_{. . k l^{\prime}}}=\frac{n_{. . k^{\prime} l}}{n_{. . k^{\prime} l^{\prime}}}
$$

Caso 4) Quando os subespaços de $B$ e $C$ forem ortogonais aos demais

$C\left(X_{\beta \mid \mu}\right) \perp C\left(X_{\left.\alpha\right|_{\mu}}\right), C\left(X_{\beta \mid \mu}\right) \perp C\left(X_{\gamma \mid \mu}\right), C\left(X_{\beta \mid \mu}\right) \perp C\left(X_{\varphi \mid \mu}\right), C\left(X_{\gamma \mid \mu}\right) \perp L C\left(X_{\left.\alpha\right|_{\mu}}\right)$, $C\left(X_{\gamma \mid \mu}\right) \perp C\left(X_{\varphi \mid \mu}\right)$, então:

$$
\frac{n_{i j .}}{n_{i j^{\prime} .}}=\frac{n_{i^{\prime} j .}}{n_{i j^{\prime} j^{\prime} .}} \text { e } \frac{n_{. j k}}{n_{. j k^{\prime}}}=\frac{n_{. j^{\prime} k}}{n_{. j^{\prime} k^{\prime}}} \text { e } \frac{n_{. \cdot j . l}}{n_{. j . l^{\prime}}}=\frac{n_{. j^{\prime} . l}}{n_{. j^{\prime} \cdot l^{\prime}}} \text { e } \frac{n_{i . k}}{n_{i . k^{\prime}}}=\frac{n_{i^{\prime} \cdot k}}{n_{i^{\prime} \cdot k^{\prime}}} \text { e } \frac{n_{. . k l}}{n_{. . k l^{\prime}}}=\frac{n_{. . k^{\prime} l}}{n_{. . k^{\prime} l^{\prime}}}
$$

Caso 5) Quando os subespaços de $B$ e $D$ forem ortogonais aos demais

$C\left(X_{\beta \mid \mu}\right) \perp C\left(X_{\alpha \mid \mu}\right), C\left(X_{\beta \mid \mu}\right) \perp C\left(X_{\gamma \mid \mu}\right), C\left(X_{\beta \mid \mu}\right) \perp L C\left(X_{\varphi \mid \mu}\right), C\left(X_{\varphi \mid \mu}\right) \perp L C\left(X_{\alpha \mid \mu}\right)$, $C\left(X_{\gamma \mid \mu}\right) \perp C\left(X_{\varphi \mid \mu}\right)$, então:

$$
\frac{n_{i j .}}{n_{i j^{\prime} .}}=\frac{n_{i^{\prime} j .}}{n_{i^{\prime} j^{\prime} .}} \text { e } \frac{n_{. j k}}{n_{. j k^{\prime}}}=\frac{n_{. j^{\prime} k}}{n_{. j^{\prime} k^{\prime}}} \text { e } \frac{n_{. j . l}}{n_{. j . l^{\prime}}}=\frac{n_{. j^{\prime} . l}}{n_{. j^{\prime} . l^{\prime}}} \text { e } \frac{n_{i . . l}}{n_{i . . l^{\prime}}}=\frac{n_{i^{\prime} . . l}}{n_{i^{\prime} . . l^{\prime}}} \text { e } \frac{n_{. . k l}}{n_{. . k l^{\prime}}}=\frac{n_{. k^{\prime} l}}{n_{. . k^{\prime} l^{\prime}}}
$$

Caso 6) Quando os subespaços de $C$ e $D$ forem ortogonais aos demais

$$
\begin{aligned}
& C\left(X_{\gamma \mid \mu}\right) . \perp C\left(X_{\alpha \mid \mu}\right), C\left(X_{\gamma \mid \mu}\right) \perp C\left(X_{\beta \mid \mu}\right), C\left(X_{\gamma \mid \mu}\right) \cdot\left\llcorner C\left(X_{\left.\varphi\right|_{\mu}}\right), C\left(X_{\varphi \mid \mu}\right) \cdot\left\llcorner C\left(X_{\alpha \mid \mu}\right),\right.\right. \\
& C\left(X_{\varphi \mid \mu}\right) \perp C\left(X_{\beta \mid \mu}\right)
\end{aligned}
$$




$$
\frac{n_{i . k}}{n_{i . k^{\prime}}}=\frac{n_{i^{\prime} . k}}{n_{i^{\prime} . k^{\prime}}} \text { e } \quad \frac{n_{. j k}}{n_{. j k^{\prime}}}=\frac{n_{. j^{\prime} k}}{n_{. j^{\prime} k^{\prime}}} \text { e } \frac{n_{. . k l}}{n_{. . k l^{\prime}}}=\frac{n_{. . k^{\prime} l}}{n_{. . k^{\prime} l^{\prime}}} \text { e } \frac{n_{i . . l}}{n_{i . . l^{\prime}}}=\frac{n_{i^{\prime} . . l}-}{n_{i^{\prime} . . l^{\prime}}} \text { e } \frac{n_{\cdot . j . l}}{n_{. j . l^{\prime}}}=\frac{n_{. j^{\prime} . l}}{n_{. j^{\prime} . l^{\prime}}}
$$

\subsubsection{Modelos com $K$ fatores}

Definição 7. Um delineamento com $K$ fatores é dito parcialmente ortogonal, se os subespaços gerados pelas colunas das submatrizes de $X$ referentes, no máximo, à $K-2$ fatores, após o ajuste de $\mu$, são individualmente ortogonais aos subespaços gerados pelas colunas das submatrizes de $X$ referentes aos outros $K-1$ fatores, após o ajuste de $\mu$.

\subsubsection{Propriedades dos delineamentos parcialmente ortogonais}

\subsection{Quanto às funções estimáveis}

a) Modelo sem interações

Para o fator ou fatores nos quais os subespaços gerados são individualmente ortogonais aos subespaços gerados pelos demais fatores, as funções estimáveis preservam as propriedades P.1, P.2, P.3, P.4 e P.5.

\section{b) Modelo com interações}

\section{b.1) Efeitos principais}

Para o fator ou fatores nos quais os subespaços gerados são individualmente ortogonais aos subespaços gerados pelos demais fatores, as funções estimáveis preservam as propriedades P.2, P.3 e P.6 


\section{b.2) Interações}

As funções estimáveis da interação entre fatores que preservam a propriedade P.8 são:

$i$ A interação que envolve conjuntamente os fatores cujos subespaços gerados não são ortogonais entre si;

ii As interações formadas pelos fatores cujos subespaços gerados foram individualmente ortogonais aos subespaços gerados pelos demais fatores; iii As interações formadas pelos casos $i$ e $i$.

\subsection{Quanto às hipóteses}

As propriedades descritas em 3.2.2.5.1 para as funções estimáveis, são mantidas para as hipóteses correspondentes.

Dado que as propriedades P.1 e P.2 são intrinsecas dos delineamentos parcialmente ortogonais, apenas as hipóteses dos tipos I e II, isto é, apenas as hipóteses sobre médias ponderadas e sobre médias ponderadas ajustadas, serão discutidas neste item. O Quadro 5 descreve as somas de quadrados, através da notação $R($.$) , conforme Searle (1971), para as$ hipóteses dos tipos I e II em modelos com até quatro fatores ortogonais. Para os modelos com mais de quatro fatores, poderão ser feitas extensões da Quadro 5 com adaptações em função da notação utilizada. 


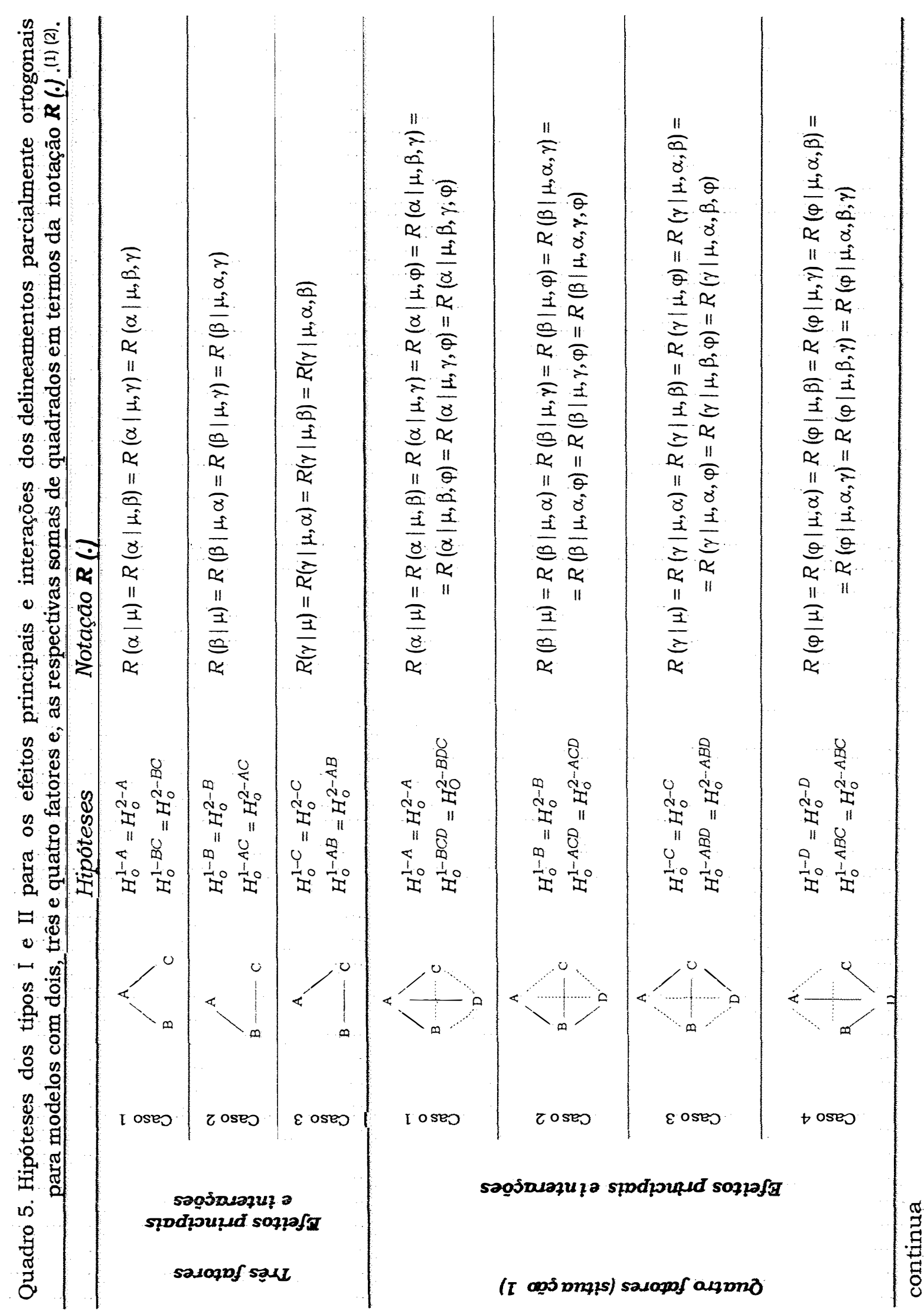




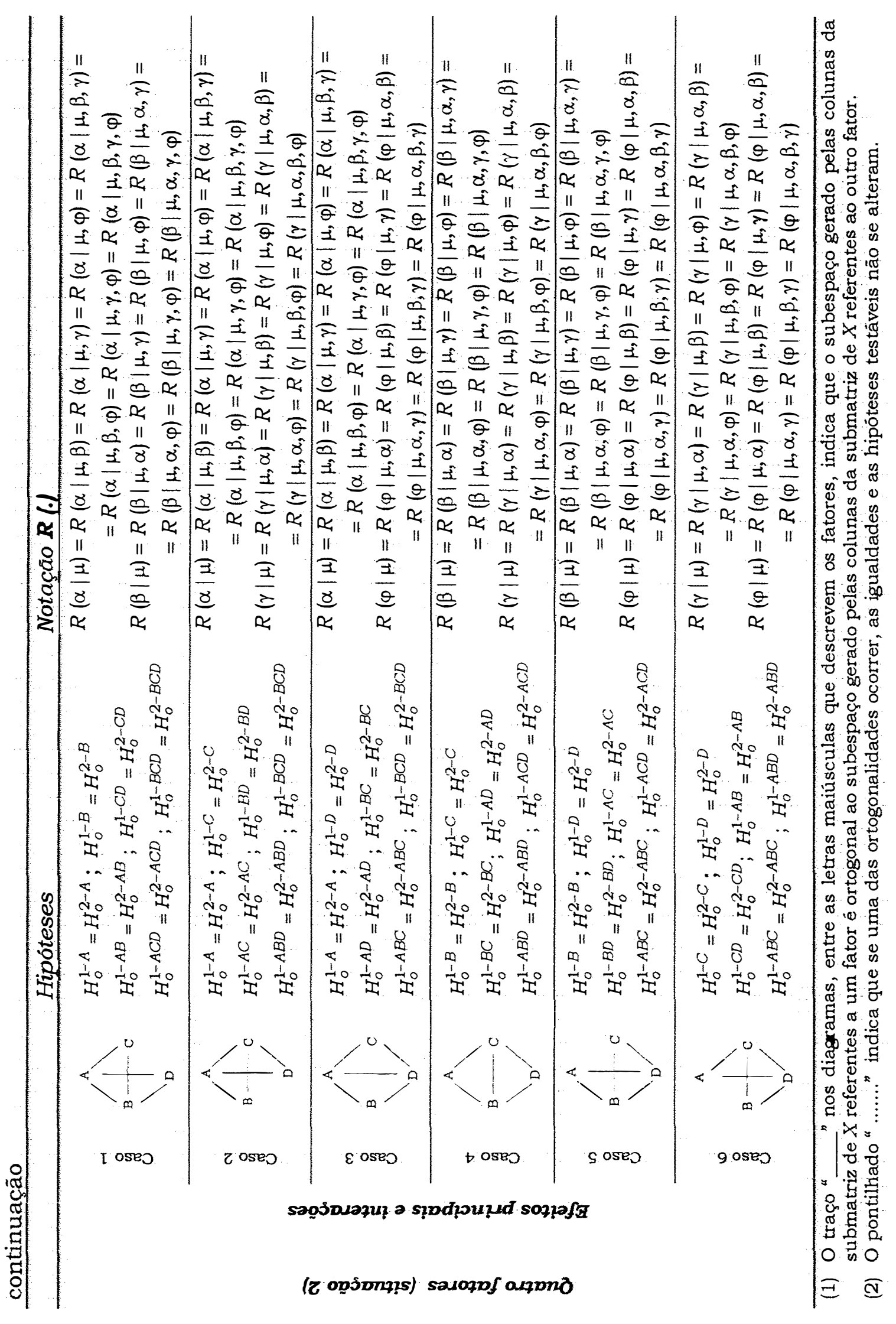




\section{RESULTADOS E DISCUSSÃO}

\subsection{Delineamentos ortogonais}

Para discussão dos delineamentos ortogonais foram utilizados modelos com dois e três fatores com e sem interações, de conjuntos balanceados e desbalanceados com freqüências de caselas proporcionais. Em todos os exemplos, enfatizou-se a propriedade inerente aos delineamentos ortogonais, de apresentarem hipóteses do tipo I iguais às hipóteses do tipo II, para todos os efeitos principais e interações do modelo. Outro aspecto ressaltado, foi a forma das funções estimáveis para os efeitos principais e interações, mostrando que nos modelos sem interações as funções estimáveis para os efeitos principais, contêm apenas os parâmetros relativos ao próprio fator e nos modelos com interações, contêm os parâmetros relativos ao próprio fator e os parâmetros das interações nas quais o fator está presente. Além disso, as funções estimáveis envolvendo interações, contêm os parâmetros relativos à própria interação e os parâmetros das interaçōes de grau maior que o graus da interação em estudo.

\subsubsection{Modelos com dois fatores}

Para discussão foi utilizado o modelo $y=X \theta+e$ caracterizado por: $y_{i j k}=\mu+\alpha_{i}+\beta_{j}+e_{i j k}$, ou seja, um modelo com dois fatores cruzados sem interação e $y_{i j k}=\mu+\alpha_{i}+\beta_{j}+\gamma_{i j}+e_{i j k}$, um modelo com dois fatores cruzados e interação, para $i=1, \ldots, a$ niveis do fator $A ; j=1, \ldots, b$ niveis do fator $B$ e $k=1, \ldots$, $n_{i j}$ observações na casela $(i ; j)$. Em todos os modelos, $y$ é um vetor de 
realizaçōes de variáveis aleatórias; $X$ é uma matriz de delineamento; $\theta$ é um vetor de parâmetros desconhecidos e e é um vetor de variáveis aleatórias nãoobserváveis, com estrutura estocástica de Gauss-Markov. Além disso, foram apresentados os quatro tipos de hipóteses, as respectivas funções estimáveis e somas de quadrados associadas incorporados pelo Proc GLM (General Linear Models Procedure) do SAS (Statistical Analysis System, 1990). Em todo o texto a igualdade apresentada entre as hipóteses dos tipos III e IV foi devida à ausência de caselas vazias, como pode ser visto em Speed, Hocking \& Hackney (1978) uma vez que conforme Iemma \& Claustriaux (1997) o termo completo é condição necessária para a ortogonalidade. Nesse contexto, a abordagem deste trabalho concentrou-se nos conjuntos completos. Na seqüência apresentam-se a forma das funçōes estimáveis e as hipóteses correspondentes, para modelos com e sem interação de conjuntos balanceados e desbalanceados provenientes de delineamentos ortogonais.

\section{i Hipóteses para o fator $A$}

i.1 Sem interação: balanceado ou desbalanceado

$H_{o}^{1-A}=H_{o}^{2-A}=H_{o}^{3-A}=H_{o}^{4-A}: \alpha_{i}=\alpha_{i^{\prime}} ; \forall\left(i, i^{\prime}\right), i \neq i^{\prime}$

i.2 Com interação, balanceado

$H_{o}^{1-A}=H_{o}^{2-A}=H_{o}^{3-A}=H_{o}^{4-A}: \alpha_{i}+\bar{\gamma}_{i .}=\alpha_{i^{\prime}}+\bar{\gamma}_{i^{\prime} .} ; \forall\left(i, i^{\prime}\right), i \neq i^{\prime}$

i. 3 Com interação, desbalanceado

$$
\begin{aligned}
& H_{o}^{1-A}=H_{o}^{2-A}: \alpha_{i}+\bar{\gamma}_{i .}=\alpha_{i^{\prime}}+\bar{\gamma}_{i^{\prime} .} ; \forall\left(i, i^{\prime}\right), i \neq i^{\prime} \\
& H_{o}^{3-A}=H_{o}^{4-A}: \alpha_{i}+\tilde{\gamma}_{i .}=\alpha_{i^{\prime}}+\tilde{\gamma}_{i^{\prime} .} ; \forall\left(i, i^{\prime}\right), i \neq i^{\prime}
\end{aligned}
$$

Naturalmente, conforme discutido em 2.2, $\bar{\gamma}$ e $\tilde{\gamma}$ descrevem, respectivamente, funçōes de médias ponderadas e de médias não ponderadas dos efeitos da interação. 


\section{ii Hipóteses para o fator B}

ii. 1 Sem interação: balanceado ou desbalanceado $H_{o}^{1-B}=H_{o}^{2-B}=H_{o}^{3-B}=H_{o}^{4-B}: \beta_{j}=\beta_{j^{\prime}} ; \forall\left(j, j^{\prime}\right), j \neq j^{\prime}$

ii.2 Com interação, balanceado $H_{o}^{1-B}=H_{o}^{2-B}=H_{o}^{3-B}=H_{o}^{4-B}: \beta_{j}+\bar{\gamma}_{. j}=\beta_{j^{\prime}}+\bar{\gamma}_{. j^{\prime}} ; \forall\left(j, j^{\prime}\right), j \neq j^{\prime}$

ii.3 Com interação, desbalanceado $H_{o}^{1-B}=H_{o}^{2-B}: \beta_{j}+\bar{\gamma}_{. j}=\beta_{j^{\prime}}+\bar{\gamma}_{. j^{\prime}} ; \forall\left(j, j^{\prime}\right), j \neq j^{\prime}$ $H_{o}^{3-B}=H_{o}^{4-B}: \beta_{j}+\tilde{\gamma}_{. j}=\beta_{j^{\prime}}+\tilde{\gamma}_{. j^{\prime}} ; \forall\left(j, j^{\prime}\right), \quad j \neq j^{\prime}$

\section{iii Hipóteses para a interação $A * B$}

Conforme anteriormente descrito na literatura:

$H_{o}^{1-A B}=H_{o}^{2-A B}=H_{o}^{3-A B}=H_{o}^{4-A B}: \gamma_{i j}-\gamma_{i^{\prime} j}-\gamma_{i j^{\prime}}+\gamma_{i^{\prime} j^{\prime}}=0 ; \quad \forall\left(i, i^{\prime}, j, j^{\prime}\right), i \neq i^{\prime}, j \neq j^{\prime}$

$\mathrm{Na}$ seqüência são apresentados alguns exemplos práticos de delineamentos ortogonais balanceados e desbalanceados com dois fatores, enfocando-se principalmente a igualdade entre as hipóteses e as respectivas somas de quadrados, além das formas das funçōes estimáveis.

\section{a Conjuntos balanceados}

\subsubsection{10 Exemplo}

Na Tabela 1 apresenta-se um exemplo clássico de delineamento ortogonal balanceado com dois fatores onde, apesar dos resultados quanto às hipóteses e as formas das funções estimáveis já serem amplamente conhecidos, serviram como uma referência indispensável para o estudo dos 
delineamentos ortogonais desbalanceados. Pode ser observado que, conforme o teorema 1,

$$
\frac{n_{i j}}{n_{i j^{\prime}}}=\frac{n_{i^{\prime} j}}{n_{i^{\prime} j^{\prime}}}=\frac{3}{3}=1
$$

e, portanto, o delineamento é ortogonal.

Tabela 1. Dados de porcentagem de germinação de canafĩstula (Peltophorum dubium Spreng) do delineamento ortogonal balanceado com dois fatores.

\begin{tabular}{|c|c|c|}
\hline \multirow[b]{2}{*}{ Substrato (fator $A$ ) } & \multicolumn{2}{|c|}{ Dormência (fator $B$ ) } \\
\hline & $j=1$ & $\dot{j} 2$ \\
\hline \multirow[t]{3}{*}{$i=1$} & 12 & 30 \\
\hline & 22 & 22 \\
\hline & 30 & 28 \\
\hline \multirow[t]{3}{*}{$i=2$} & 38 & 12 \\
\hline & 46 & 15 \\
\hline & 42 & 26 \\
\hline
\end{tabular}

\section{a.1 Modelo sem interação}

Nos delineamentos ortogonais balanceados de modelos sem interação, ocorre a igualdade entre os quatro tipos de hipóteses fornecidos pelo Proc GLM do SAS. Para o exemplo da Tabela 1 são ilustradas as funções estimáveis dos tipos I e II apenas para o fator $A$ (Quadro 6), ressaltando a propriedade dos delineamentos ortogonais, P.1, de apresentar apenas parâmetros relativos ao próprio fator para as funções estumáveis de efeitos principais, na ausência de interação. 
Quadro 6. Funçōes estimáveis fornecidas pelo Proc GLM do SAS e respectivas hipóteses sobre o fator $A$, para os dados da Tabela 1 , considerando o modelo sem interaçāo.

\begin{tabular}{|c|c|c|c|c|c|}
\hline \multicolumn{3}{|c|}{ Tipo I-A $=$ Tipo II-A } & \multicolumn{3}{|c|}{ Tipo III-A = Tipo IV-A } \\
\hline \multicolumn{2}{|c|}{$\begin{array}{l}\text { Efeito } \\
\text { INTERCEPTO }\end{array}$} & $\begin{array}{l}\text { Coeficientes } \\
0\end{array}$ & \multicolumn{2}{|c|}{$\begin{array}{l}\text { 三eito } \\
\text { sitereEto }\end{array}$} & \multirow{2}{*}{$\begin{array}{l}\text { Coeficientes } \\
0 \\
\mathrm{~L} 2 \\
-\mathrm{L} 2\end{array}$} \\
\hline A & $\begin{array}{l}1 \\
2\end{array}$ & $\begin{array}{l}\mathrm{L} 2 \\
-\mathrm{I} 2\end{array}$ & 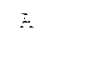 & $\begin{array}{l}1 \\
2\end{array}$ & \\
\hline B & $\begin{array}{l}1 \\
2\end{array}$ & $\begin{array}{l}0 \\
0\end{array}$ & $\Xi$ & $\begin{array}{l}1 \\
2\end{array}$ & $\begin{array}{l}0 \\
0\end{array}$ \\
\hline & & & $\alpha_{1}=\alpha_{2}$ & & \\
\hline
\end{tabular}

A análise de variância (Tabela 2) mostra a igualdade entre as somas de quadrados associadas às hipóteses dos tipos I e II, P.2, para o fator $A$, ou seja, $R(\alpha \mid \mu)=R(\alpha \mid \mu, \beta)=40,04167$. Para que a igualdade seja verificada para o fator $B, R(\beta \mid \mu)=R(\beta \mid \mu, \alpha)$, deve-se inverter a ordem de entrada dos fatores no modelo. A igualdade entre as somas de quadrados associadas às hipóteses dos tipos II e III foi motivada pela ausência de interação, P.4, e a igualdade entre as somas de quadrados associadas às hipóteses dos tipos III e IV é devida à ausência de caselas vazias, P.3, uma vez que o conjunto é completo.

Tabela 2. Análise de variância do delineamento ortogonal balanceado, descrito na Tabela 1 , segundo o modelo $y_{i j k}=\mu+\alpha_{i}+\beta_{j}+e_{i j k}$, declarando $A$ como primeiro fator.

\begin{tabular}{lcrrl}
\hline Fontes & $g l$ & S.Q. TipoI & $Q . M$ & $R()$. \\
\hline A & 1 & 40,04166667 & 40,04166667 & $\boldsymbol{R}(\alpha \mid \mu)$ \\
B & 1 & 1717,04166667 & $: 717,04166667$ & $R(\beta \mid \mu, \alpha)$ \\
\hline Fontes & $g l$ & S.Q.Tipo II & $Q . M$. & $R()$. \\
\hline A & 1 & $\mathbf{4 0 , 0 4 1 6 6 6 6 7}$ & 40,04166667 & $\boldsymbol{R}(\alpha \mid \mu, \beta)$ \\
B & 1 & 1717,04166667 & $: 717,04166667$ & $R(\beta \mid \mu, \alpha)$ \\
\hline Fontes & $g l$ & S.Q.Tipo III=IV & Q.M. & $R(\cdot)$ \\
\hline A & 1 & 40,04166667 & 40,04166667 & $\boldsymbol{R}(\dot{\alpha} \mid \dot{\mu}, \dot{\beta})$ \\
B & 1 & 1717,04166667 & 1717,04166667 & $R(\dot{\beta} \mid \dot{\mu}, \dot{\alpha})$ \\
\hline
\end{tabular}


Como os conjuntos balanceados encontram-se amplamente discutidos na literatura, a verificação da igualdade entre as hipóteses dos tipos I e II, com a inversão na ordem de entrada dos. fatores no modelo, para os efeitos principais do modelo, está abordada com mais detalhes no conjunto desbalanceado.

\section{a.2 Modelo com interação}

Para os delineamentos ortogonais balanceados de modelos com interação, os resultados são idênticos aos modelos sem interação em termos de igualdade de hipóteses, ou seja, ocorre também a igualdade entre os quatro tipos de hipóteses fornecidos pelo Proc GLM do SAS. A diferença entre os modelos sem interação e com interação consiste no fato de que as funções estimáveis envolvendo um único fator do modelo com interação apresentam além dos parâmetros do próprio fator, parâmetros da interação, como pode ser observado no Quadro 7.

Quadro 7. Funções estimáveis fornecidas pelo Proc GLM do SAS e respectivas hipóteses sobre o fator $A$, para os dados da Tabela 1 , considerando $o$ modelo com interação.

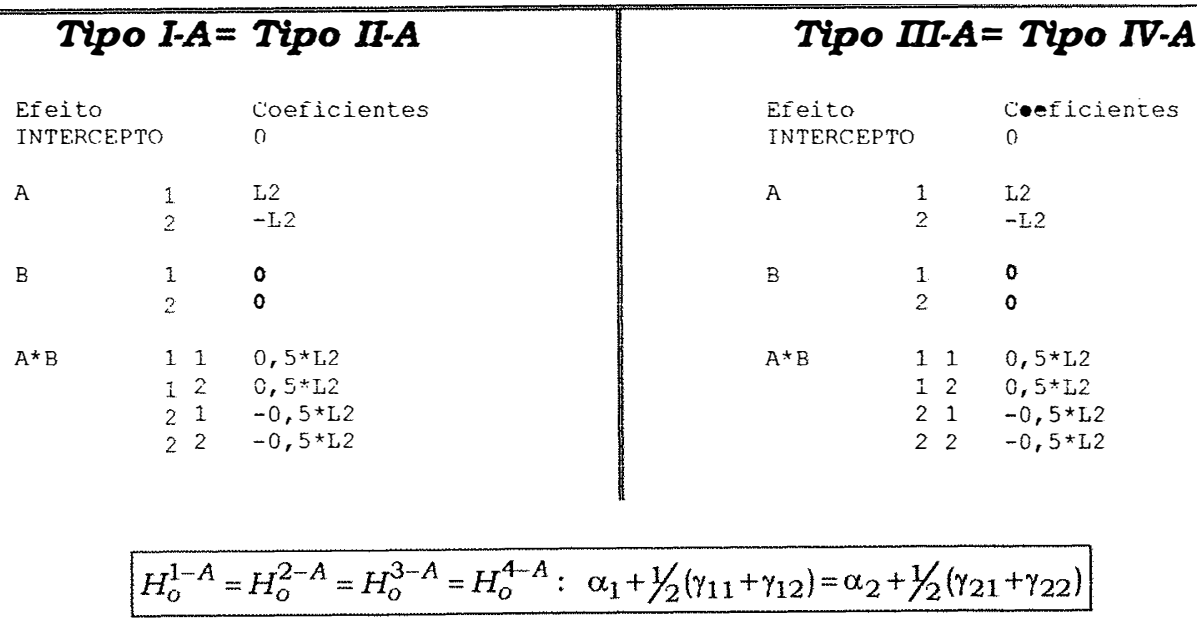


Na Tabela 3 observa-se uma caracteristica muito particular dos conjuntos balanceados, de apresentar igualdade entre as somas de quadrados associadas às hipóteses dos tipos II e III, $R(\alpha \mid \mu, \beta)=R(\dot{\alpha} \mid \dot{\mu}, \dot{\beta})=40,0147$ e $R(\beta \mid \mu, \alpha)=R(\dot{\beta} \mid \dot{\mu}, \dot{\alpha})=1717,0417$, mesmo quando o modelo contém interação.

Tabela 3. Análise de variância do delineamento ortogonal balanceado, descrito na Tabela 1 , segundo o modelo $y_{i j k}=\mu+\alpha_{i}+\beta_{j}+\gamma_{i j}+e_{i j k}$, declarando $A$ como primeiro fator.

\begin{tabular}{|c|c|c|c|c|}
\hline Fontes & $g l$ & SQ Tipo I & Q.M. & $R()$. \\
\hline A & 1 & 40,041666667 & 40,041666667 & $\boldsymbol{R}(\alpha \mid \mu)$ \\
\hline B & 1 & 1717,04166667 & 1717,04166667 & $R(\beta \mid \mu, \alpha)$ \\
\hline$A^{\star} B$ & 1 & 92,04166667 & 92,04166667 & $R(\gamma \mid \mu, \alpha, \beta)$ \\
\hline Fontes & $g l$ & SQ Tipo II & Q.M. & $R()$. \\
\hline A & 1 & 40,04166667 & 40,04165657 & $\boldsymbol{R}(\alpha \mid \mu, \beta)$ \\
\hline B & 1 & 1717,04166667 & 1717,04166667 & $R(\beta \mid \mu, \alpha)$ \\
\hline$A * B$ & 1 & 92,04166667 & 92,04166667 & $R(\gamma \mid \mu, \alpha, \beta)$ \\
\hline Fontes & $g l$ & SQ Tipo $I I I=I V$ & Q.M. & $R()$. \\
\hline A & 1 & 40,04166567 & 40,04166667 & $\boldsymbol{R}(\dot{\alpha} \mid \dot{\mu}, \dot{\beta})$ \\
\hline B & 1 & 1717,04166667 & 1717,04166667 & $R(\dot{\beta} \mid \dot{\mu}, \dot{\alpha})$ \\
\hline$A \star B$ & 1 & 92,04166667 & 92,04166667 & $R(\dot{\gamma} \mid \dot{\mu}, \dot{\alpha}, \dot{\beta})$ \\
\hline
\end{tabular}

\section{b Conjuntos desbalanceados}

\subsubsection{2o Exemplo}

A discussão do exemplo da Tabela 4 retrata alguns resultados importantes quanto a igualdade de hipóteses e a forma das funções estimáveis de delineamentos ortogonais desbalanceados, com freqüências de caselas proporcionais para modelos com dois fatores, já apresentados e discutidos por Claustriaux \& Iemma (1999). Sendo assim, foram abordados apenas os aspectos mais relevantes para dar ao texto uma seqüência didática. 
Tabela 4. Dados de porcentagem de germinação de canafístula (Peltophorum dubium Spreng) do delineamento ortogonal desbalanceado com dois fatores.

\begin{tabular}{|c|c|c|}
\hline \multirow[b]{2}{*}{ Substrato ( fator $A$ ) } & \multicolumn{2}{|c|}{ Dormência ( fator $B$ ) } \\
\hline & $\dot{j}=1$ & $\dot{j}=2$ \\
\hline \multirow[t]{6}{*}{$i=1$} & 12 & 30 \\
\hline & 22 & 22 \\
\hline & 30 & \\
\hline & 12 & \\
\hline & 18 & \\
\hline & 38 & \\
\hline \multirow[t]{3}{*}{$i=2$} & 38 & 12 \\
\hline & 46 & \\
\hline & 42 & \\
\hline
\end{tabular}

Segundo Iemma \& Claustriaux (1997), observa-se no teorema 1 que uma condição necessária e suficiente para que os subespaços gerados pelas submatrizes de $X$ referentes a cada fator, após o ajuste de $\mu$, sejam ortogonais e, portanto, o delineamento seja ortogonal, é que as freqüências das caselas sejam proporcionais. A expressão da proporcionalidade foi apresentada em (5):

$$
\frac{n_{i j}}{n_{i j^{\prime}}}=\frac{n_{i^{\prime} j}}{n_{i^{\prime} j^{\prime}}}, \forall\left(i \neq i^{\prime}, j \neq j^{\prime}\right)
$$

Verificando-se o teorema para os dados da Tabela 4, obteve-se a seguinte proporção:

$$
\frac{n_{11}}{n_{12}}=\frac{n_{21}}{n_{22}}=3, \quad \forall\left(i \neq i^{\prime}, j \neq j^{\prime}\right)
$$

Essa proporcionalidade garante que, apesar do desbalanceamento, as hipóteses do tipo I são iguais às hipóteses do tipo II para os efeitos principais e interação, caracterizando o delineamento como ortogonal.

Não se pode perder de vista que nos delineamentos com dois fatores cruzados, a hipótese sobre a interação é a mesma para os quatro tipos, conforme descrito por Speed, Hocking e Hackney (1978). Generalizações importantes são feitas nos próximos itens, para delineamentos com mais de dois fatores. 


\section{b.1 Modelo sem interação}

A análise dos dados através do modelo com dois fatores sem interação mostra, no Quadro 8, que apesar do desbalanceamento, as hipóteses dos tipos I e II são iguais, P.2, e as funções estimáveis de um fator não apresentaram parâmetros relativos ao outro fator, P.1. Essa característica dos delineamentos ortogonais desbalanceados é a mesma dos conjuntos balanceados, onde se concentram as hipóteses de maior interesse do pesquisador. Em termos práticos, implica que as hipóteses testadas nos conjuntos balanceados com dois fatores cruzados sem interação, têm as mesmas caracteristicas das hipóteses testadas nos conjuntos desbalanceados com freqüências proporcionais nas caselas.

Quadro 8. Funções estimáveis fornecidas pelo Proc GLM do SAS e respectivas hipóteses sobre o fator $A$, para os dados da Tabela 4, considerando o modelo sem interação.

\begin{tabular}{|c|c|c|c|c|}
\hline \multicolumn{3}{|c|}{ Tipo I-A = Tipo II-A } & \multicolumn{2}{|c|}{ Tipo III-A = Tipo IV-A } \\
\hline \multicolumn{2}{|c|}{$\begin{array}{l}\text { Ef́eito } \\
\text { INTERCEPTO }\end{array}$} & $\begin{array}{l}\text { Coeficientes } \\
0\end{array}$ & $\begin{array}{l}\text { EZeito } \\
\text { ZirERCEPTO }\end{array}$ & \multirow{2}{*}{$\begin{array}{l}\text { Coeficientes } \\
0 \\
\mathrm{~L} 2 \\
-\mathrm{I} 2\end{array}$} \\
\hline A & $\begin{array}{l}1 \\
2\end{array}$ & $\begin{array}{l}\mathrm{I} 2 \\
-\mathrm{I} 2\end{array}$ & $\therefore$ & \\
\hline B & $\begin{array}{l}1 \\
2\end{array}$ & $\begin{array}{l}0 \\
0\end{array}$ & $\equiv$ & $\begin{array}{l}0 \\
0\end{array}$ \\
\hline & & $H_{o}^{1-A}$ & $\alpha_{1}=\alpha_{2}$ & \\
\hline
\end{tabular}

A análise de variância da Tabela 5 mostra, através da notação $R($ ), a igualdade entre as somas de quadrados associadas às hipóteses dos tipos I e II para o fator $A$, ou seja, $R(\alpha \mid \mu)=R(\alpha \mid \mu, \beta)=352,6667$. Para verificar que tais resultados também ocorreram para o fator $B$, inverteu-se a ordem de entrada dos fatores no modelo, declarando $B$ como o primeiro fator (Tabela 6), mostrando também que: $R(\beta \mid \mu)=R(\beta \mid \mu, \alpha)=121,0000$. A igualdade entre as somas de quadrados associadas às hipóteses dos tipos II e III é devida à 
ausência de interação no modelo, assim, $R(\alpha \mid \mu, \beta)=R(\dot{\alpha} \mid \dot{\mu}, \dot{\beta})=352,6667$ e $R(\beta \mid \mu, \alpha)=R\left(\left.\dot{\beta}\right|_{\mu}, \dot{\alpha}\right)=121,0000$.

Tabela 5. Análise de variância do delineamento ortogonal desbalanceado, descrito na Tabela 4 , segundo o modelo $y_{i j k}=\mu+\alpha_{i}+\beta_{j}+e_{i j k}$, declarando $A$ como primeiro fator.

\begin{tabular}{lcrrl}
\hline Fontes & $g l$ & SQ Tipo I & Q.M. & $R(\cdot)$ \\
\hline A & 1 & 352,66666667 & 352,66666667 & $R(\alpha \mid \mu)$ \\
B & 1 & 121,00000000 & 121,00000000 & $R(\beta \mid \mu, \alpha)$ \\
\hline Fontes & $g l$ & SQ Tipo II & Q.M. & $R()$. \\
\hline A & 1 & 352,66666667 & $352,5 \varepsilon \epsilon 5 \leqslant 667$ & $\boldsymbol{R}(\alpha \mid \mu, \beta)$ \\
B & 1 & 121,00000000 & 121,00000000 & $R(\beta \mid \mu, \alpha)$ \\
\hline Fontes & $g l$ & SQ Tipo III=IV & $Q . M$. & $R(\cdot)$ \\
\hline A & 1 & $\mathbf{3 5 2 , 6 6 6 6 6 6 6 7}$ & 352,66665667 & $\boldsymbol{R}(\dot{\alpha} \mid \dot{\mu}, \dot{\beta})$ \\
B & 1 & 121,00000000 & 121,00000000 & $R(\dot{\beta} \mid \dot{\mu}, \dot{\alpha})$ \\
\hline
\end{tabular}

Tabela 6. Análise de variância do delineamento ortogonal desbalanceado, descrito na Tabela 4 , segundo o modelo $y_{i j k}=\mu+\beta_{j}+\alpha_{i}+e_{i j k}$, declarando $B$ como primeiro fator.

\begin{tabular}{lcrrl}
\hline Fontes & $g l$ & SQ Tipo I & Q.M. & $R()$. \\
\hline B & 1 & 121,00000000 & 121,00000000 & $\boldsymbol{R}(\beta \mid \mu)$ \\
A & 1 & 352,66666667 & 352,66656567 & $R(\alpha \mid \mu, \beta)$ \\
\hline Fontes & $g l$ & SQ Tipo II & $Q . M$. & $R()$. \\
\hline B & 1 & 121,00000000 & 121,00000000 & $\boldsymbol{R}(\beta \mid \mu, \alpha)$ \\
A & 1 & 352,66666667 & 352,66666667 & $R(\alpha \mid \mu, \beta)$ \\
\hline Fontes & $g l$ & SQ Tipo II =IV & $Q . M$. & $R()$. \\
\hline B & 1 & 121,00000000 & 121,00000000 & $R(\dot{\beta} \mid \dot{\mu}, \dot{\alpha})$ \\
A & 1 & 352,66666667 & 352,66666567 & $R(\dot{\alpha} \mid \dot{\mu}, \dot{\beta})$ \\
\hline
\end{tabular}

\section{b.2 Modelo com interação}

Nos modelos com dois fatores e interação, a forma das funções estimáveis envolvendo um único fator é a mesma dos conjuntos balanceados. As funções estimáveis para o fator $A$ apresentaram parâmetros relativos ao 
próprio fator, parâmetros da interação e não apresentaram parâmetros relativos ao outro fator (Quadro 9).

Quadro 9. Funçōes estimáveis fornecidas pelo Proc GLM do SAS e respectivas hipóteses sobre o fator $A$, para os dados da Tabela 4, considerando o modelo com interação.

\begin{tabular}{|c|c|c|c|c|c|}
\hline \multicolumn{3}{|c|}{ Tipo I-A = Tipo II-A } & \multicolumn{3}{|c|}{ Tipo III-A = Tipo IV-A } \\
\hline $\begin{array}{l}\text { Efe } \\
\text { INTY }\end{array}$ & & $\begin{array}{l}\text { Coefítientes } \\
0\end{array}$ & \multicolumn{2}{|c|}{$\begin{array}{l}\text { Efeito } \\
\text { INTERCEPTO }\end{array}$} & $\begin{array}{l}\text { Coeficientes } \\
0\end{array}$ \\
\hline A & $\begin{array}{l}1 \\
2\end{array}$ & $\begin{array}{ll}\mathrm{L}, 2 \\
-\mathrm{L} 2\end{array}$ & A & & $\begin{array}{l}\mathrm{L} 2 \\
-\mathrm{L} 2\end{array}$ \\
\hline B & & $\stackrel{0}{0}$ & B & & $:$ \\
\hline$A \star B$ & $\begin{array}{ll}1 & 1 \\
1 & 2 \\
2 & 1 \\
2 & 2\end{array}$ & $\begin{array}{l}0,75 * \mathrm{~L} 2 \\
0,25 *_{\mathrm{L} 2} \\
-0,75 * \mathrm{~L} 2 \\
-0,25 * \mathrm{~L} 2\end{array}$ & $A^{\star}{ }^{\prime}$ & $\begin{array}{lll}1 & 1 \\
1 & 2 \\
2 & 1 \\
2 & 2\end{array}$ & $\begin{array}{l}0,5 * \mathrm{~L} 2 \\
0,5 \times 522 \\
-0,5 * \mathrm{~L} 2 \\
-0,5 * \mathrm{~L} 2\end{array}$ \\
\hline & & \multicolumn{3}{|c|}{$H_{o}^{1-A}=H_{o}^{2-A}: \alpha_{1}+1 / 4\left(3 \gamma_{11}+\gamma_{12}\right)=\alpha_{2}+1 / 4\left(3 \gamma_{21}+\gamma_{22}\right)$} & \\
\hline & & \multicolumn{3}{|c|}{$H_{o}^{3-A}=H_{o}^{4-A}: \alpha_{1}+1 / 2\left(\gamma_{11}+\gamma_{12}\right)=\alpha_{2}+1 / 2\left(\gamma_{21}+\gamma_{22}\right)$} & \\
\hline
\end{tabular}

Diferentemente dos delineamentos ortogonais balanceados com interação, os delineamentos ortogonais desbalanceados com interação, não apresentam igualdade entre os quatro tipos de hipóteses fornecidos Proc GLM do SAS. São iguais as somas de quadrados associadas às hipóteses dos tipos I e II para os fatores $A$ e $B$, pela ortogonalidade e as hipóteses dos tipos III e IV, pela ausência de caselas vazias. Consequentemente $R(\alpha \mid \mu)=R(\alpha \mid \mu, \beta)$ e $R(\beta \mid \mu)=R(\beta \mid \mu, \alpha)$ mas, a hipótese do tipo II é diferente da hipótese do tipo III na presença de interação com desbalanceamento (Tabela 7), ou seja, $R(\alpha \mid \mu, \beta) \neq R(\dot{\alpha} \mid \dot{\mu}, \dot{\beta})$ e $R(\beta \mid \mu, \alpha) \neq R(\dot{\beta} \mid \dot{\mu}, \dot{\alpha})$. 
Tabela 7. Análise de variância do delineamento ortogonal desbalanceado, descrito na Tabela 4, segundo o modelo $y_{i j k}=\mu+\alpha_{i}+\beta_{j}+\gamma_{i j}+e_{i j k}$, declarando A como primeiro fator.

\begin{tabular}{|c|c|c|c|c|}
\hline Fontes & $g l$ & SQ Tipo I & Q.M. & $R()$. \\
\hline A & 1 & 352,66666667 & 352,66666667 & $\boldsymbol{R}(\alpha \mid \mu)$ \\
\hline B & 1 & 121,00000000 & 121,00000000 & $R(\beta \mid \mu, \alpha)$ \\
\hline$A \star B$ & 1 & 578,00000000 & 578,00000000 & $R(\gamma \mid \mu, \alpha, \beta)$ \\
\hline Fontes & $g l$ & SQ Tipo II & Q.M. & $R()$. \\
\hline A & 1 & 352,66666667 & 352,66666667 & $\boldsymbol{R}(\alpha \mid \mu, \beta)$ \\
\hline B & 1 & 121,00000000 & 121,00000000 & $R(\beta \mid \mu, \alpha)$ \\
\hline$A * B$ & 1 & 578,00000000 & 578,00000000 & $R(\gamma \mid \mu, \alpha, \beta)$ \\
\hline Fontes & $g l$ & SQ Tipo $I I=I V$ & Q.M. & $R()$. \\
\hline$A$ & 1 & 18,00000000 & 10,00000000 & $\boldsymbol{R}(\dot{\alpha} \mid \dot{\mu}, \dot{\beta})$ \\
\hline B & 1 & 338,00000000 & 338,00000000 & $R(\dot{\beta} \mid \dot{\mu}, \dot{\alpha})$ \\
\hline$A * B$ & 1 & 578,00000000 & 578,00000000 & $R(\dot{\gamma} \mid \dot{\mu}, \dot{\alpha}, \dot{\beta})$ \\
\hline
\end{tabular}

\subsubsection{Modelos com três fatores}

Para a discussão adotou-se o modelo $y=X \theta+e$, caracterizado por: $y_{i j k l}=\mu+\alpha_{i}+\beta_{j}+\gamma_{k}+e_{i j k l}$, ou seja, um modelo com três fatores sem interações e por: $y_{i j k l}=\mu+\alpha_{i}+\beta_{j}+\gamma_{k}+\varphi_{i j}+\eta_{i k}+\tau_{j k}+\rho_{i j k}+e_{i j k l}$, um modelo com três fatores e interações, para $i=1, \ldots, a$ niveis do fator $A ; j=1, \ldots, b$ niveis do fator $B, k=1, \ldots, c$ niveis do fator $C$ e $l=1, \ldots, n_{i j k}$ observações de cada casela $(i ; j ; k)$.

A forma das hipóteses e, portanto, das funções estimáveis nos modelos com e sem interações está apresentada a seguir:

\section{$i$ Hipóteses para o fator $A$}

i. 1 Sem interações: balanceado ou desbalanceado

$H_{o}^{1-A}=H_{o}^{2-A}=H_{o}^{3-A}=H_{o}^{4-A}: \alpha_{i}=\alpha_{i^{\prime}} ; \forall\left(i, i^{\prime}\right), i \neq i^{\prime}$

i.2 Com interações, balanceado

$H_{o}^{1-A}=H_{o}^{2-A}=H_{o}^{3-A}=H_{o}^{4-A}: \alpha_{i}+\bar{\varphi}_{i .}+\bar{\eta}_{i .}+\bar{\rho}_{i . .}=\alpha_{i^{\prime}}+\bar{\varphi}_{i^{\prime} .}+\bar{\eta}_{i^{\prime} .}+\bar{\rho}_{i^{\prime} . .} ; \forall\left(i, i^{\prime}\right), i \neq i^{\prime}$ 
i.3 Com interações, desbalanceado $H_{o}^{1-A}=H_{o}^{2-A}: \alpha_{i}+\bar{\varphi}_{i .}+\bar{\eta}_{i .}+\bar{\rho}_{i . .}=\alpha_{i^{\prime}}+\bar{\varphi}_{i^{\prime} .}+\bar{\eta}_{i^{\prime} .}+\bar{\rho}_{i^{\prime} .} ; \forall\left(i, i^{\prime}\right), i \neq i^{\prime}$ $H_{o}^{3-A}=H_{0}^{4-A}: \alpha_{i}+\tilde{\varphi}_{i .}+\tilde{\eta}_{i .}+\tilde{\rho}_{i . .}=\alpha_{i^{\prime}}+\tilde{\varphi}_{i^{\prime} .}+\tilde{\eta}_{i^{\prime} .}+\tilde{\rho}_{i^{\prime} .} ; \forall\left(i, i^{\prime}\right), i \neq i^{\prime}$

\section{ii Hipóteses para o fator $B$}

ii.1 Sem interações, balanceado ou desbalanceado $H_{o}^{1-B}=H_{o}^{2-B}=H_{o}^{3-B}=H_{o}^{4-B}: \beta_{j}=\beta_{j^{\prime}} ; \forall\left(j, j^{\prime}\right), j \neq j^{\prime}$

ii.2 Com interações, balanceado $H_{o}^{1-B}=H_{o}^{2-B}=H_{o}^{3-B}=H_{o}^{4-B}: \beta_{j}+\bar{\varphi}_{. j}+\bar{\tau}_{j .}+\bar{\rho}_{. j .}=\beta_{j^{\prime}}+\bar{\varphi}_{. j^{\prime}}+\bar{\tau}_{j^{\prime} .}+\bar{\rho}_{. j .} ; \forall\left(j, j^{\prime}\right), j \neq j^{\prime}$

ii.3 Com interações, desbalanceado $H_{o}^{1-B}=H_{o}^{2-B}: \beta_{j}+\bar{\varphi}_{. j}+\bar{\tau}_{j .}+\bar{\rho}_{. j .}=\beta_{j^{\prime}}+\bar{\varphi}_{. j^{\prime}}+\bar{\tau}_{j^{\prime} .}+\bar{\rho}_{. j .} ; \forall\left(j, j^{\prime}\right), j \neq j^{\prime}$
$H_{o}^{3-B}=H_{o}^{4-B}: \beta_{j}+\tilde{\varphi}_{. j}+\tilde{\tau}_{j .}+\tilde{\rho}_{. j .}=\beta_{j^{\prime}}+\tilde{\varphi}_{. j^{\prime}}+\tilde{\tau}_{j^{\prime} .}+\tilde{\rho}_{. j .} ; \forall\left(j, j^{\prime}\right), j \neq j^{\prime}$

iii Hipóteses para o fator C

iii.1 Sem interações: balanceado ou desbalanceado $H_{o}^{1-C}=H_{o}^{2-C}=H_{o}^{3-C}=H_{o}^{4-C}: \gamma_{k}=\gamma_{k^{\prime}} ; \forall\left(k, k^{\prime}\right), k \neq k^{\prime}$

iii.2 Com interações, balanceado $H_{o}^{1-C}=H_{o}^{2-C}=H_{o}^{3-C}=H_{o}^{4-C}: \gamma_{k}+\bar{\eta}_{. k}+\bar{\tau}_{. k}+\bar{\rho}_{. . k}=\gamma_{k^{\prime}}+\bar{\eta}_{. k^{\prime}}+\bar{\tau}_{. k^{\prime}}+\bar{\rho}_{. . k^{\prime}} ; \forall\left(k, k^{\prime}\right), \quad k \neq k^{\prime}$ iii. 3 Com interações, desbalanceado $H_{o}^{1-C}=H_{o}^{2-C}: \gamma_{k}+\bar{\eta}_{. k}+\bar{\tau}_{. k}+\bar{\rho}_{. . k}=\gamma_{k^{\prime}}+\bar{\eta}_{. k^{\prime}}+\bar{\tau}_{. k^{\prime}}+\bar{\rho}_{. . k^{\prime}} ; \forall\left(k, k^{\prime}\right), \quad k \neq k^{\prime}$ $H_{o}^{3-C}=H_{o}^{4-C}: \gamma_{k}+\tilde{\eta}_{. k}+\tilde{\tau}_{. k}+\tilde{\rho}_{. . k}=\gamma_{k^{\prime}}+\tilde{\eta}_{. k^{\prime}}+\tilde{\tau}_{. k^{\prime}}+\tilde{\rho}_{. . k^{\prime}} ; \forall\left(k, k^{\prime}\right), k \neq k^{\prime}$ 


\section{iii Hipóteses para a interação $A^{*} B$}

iii.1 Balanceado

$$
\begin{gathered}
H_{o}^{1-A B}=H_{o}^{2-A B}=H_{o}^{3-A B}=H_{o}^{4-A B}: \varphi_{i j}-\varphi_{i j^{-}}-\varphi_{i^{-} j}+\varphi_{i^{-} j^{-}}+\bar{\rho}_{i j .}-\bar{\rho}_{i^{-} j .}-\bar{\rho}_{i j^{-} .}+\bar{\rho}_{i^{-} j^{-} .}=0 \\
\forall\left(i, i^{\prime}, j, j^{\prime}\right), i \neq i^{\prime}, j \neq j^{\prime}
\end{gathered}
$$

iii.2 Desbalanceado

$$
\begin{aligned}
& H_{o}^{1-A B}=H_{o}^{2-A B}: \varphi_{i j}+-\varphi_{i j^{-}}-\varphi_{i^{-j} j}+\varphi_{i^{\prime} j^{-}}+\bar{\rho}_{i j .}-\bar{\rho}_{i^{-j} j .}-\bar{\rho}_{i j^{\prime} .}+\bar{\rho}_{i^{\prime} j^{-} .}=0 ; \\
& \forall\left(i, i^{\prime}, j, j^{\prime}\right), i \neq i^{\prime}, j \neq j^{\prime} \\
& H_{o}^{3-A B}=H_{o}^{4-A B}: \varphi_{i j}+-\varphi_{i j^{-}}-\varphi_{i^{-} j}+\varphi_{i^{-} j^{-}}+\tilde{\rho}_{i j .}-\tilde{\rho}_{i^{-} j .}-\tilde{\rho}_{i j^{\prime} .}+\tilde{\rho}_{i^{-} j^{-} .}=0 ; \\
& \forall\left(i, i^{\prime}, j, j^{\prime}\right), i \neq i^{\prime}, j \neq j^{\prime}
\end{aligned}
$$

\section{iv Hipóteses para a interação $A^{*} C$}

iv. 1 Balanceado

$$
\begin{aligned}
H_{o}^{1-A C}=H_{o}^{2-A C}=H_{o}^{3-A C}=H_{o}^{4-A C}: & \eta_{i k}-\eta_{i k^{-}}-\eta_{i^{\prime} k}+\eta_{i^{-} k^{-}}+\bar{\rho}_{i . k}-\bar{\rho}_{i^{-} . k}-\bar{\rho}_{i . k^{-}}+\bar{\rho}_{i^{\prime} \cdot k^{-}}=0 \\
& \forall\left(i, i^{\prime}, k, k^{\prime}\right), i \neq i^{\prime}, k \neq k^{\prime}
\end{aligned}
$$

iv.2 Desbalanceado

$$
\begin{aligned}
& H_{o}^{1-A C}=H_{o}^{2-A C}: \eta_{i k^{-}}-\eta_{i k^{-}}-\eta_{i^{-k} k}+\eta_{i^{-k^{-}}}+\bar{\rho}_{i . k}-\bar{\rho}_{i^{-} . k}-\bar{\rho}_{i . k^{-}}+\bar{\rho}_{i^{*} . k^{-}}=0 \\
& \forall\left(i, i^{\prime}, k, k^{\prime}\right), i \neq i^{\prime}, k \neq k^{\prime} \\
& H_{o}^{3-A C}=H_{o}^{4-A C}: \eta_{i k^{-}}-\eta_{i k^{-}}-\eta_{i^{-k} k^{\prime}}+\eta_{i^{-} k^{-}}+\tilde{\rho}_{i \cdot k}-\tilde{\rho}_{i^{*} \cdot k}-\tilde{\rho}_{i . k^{-}}+\tilde{\rho}_{i^{-} \cdot k^{-}}=0 \\
& \forall\left(i, i^{\prime}, k, k^{\prime}\right), \quad i \neq i^{\prime}, k \neq k^{\prime}
\end{aligned}
$$

$v$ Hipóteses para a interação $B^{*} C$

v. 1 Balanceado

$$
\begin{aligned}
H_{o}^{1-B C}=H_{o}^{2-B C}=H_{o}^{3-B C}=H_{o}^{4-B C} & : \tau_{j k}-\tau_{j k^{-}}-\tau_{j^{\prime} k}+\tau_{j^{\prime} k^{-}}+\bar{\rho}_{. j k}-\bar{\rho}_{. j^{\prime} k}-\bar{\rho}_{. j k^{-}}+\bar{\rho}_{. j^{\prime} k^{-}}=0 \\
& \forall\left(j, j^{\prime}, k, k^{\prime}\right), j \neq j^{\prime}, k \neq k^{\prime}
\end{aligned}
$$


v.2 Desbalanceado

$$
\begin{gathered}
H_{o}^{1-B C}=H_{o}^{2-B C}: \tau_{j k}-\tau_{j k^{-}}-\tau_{j^{\prime} k}+\tau_{j^{\prime} k^{-}}+\bar{\rho}_{. j k}-\bar{\rho}_{. j^{\prime} k}-\bar{\rho}_{. j k^{-}}+\bar{\rho}_{. j^{\prime} k^{-}}=0 \\
\forall\left(j, j^{\prime}, k, k^{\prime}\right), j \neq j^{\prime}, k \neq k^{\prime} \\
\begin{aligned}
H_{o}^{2-B C}=H_{o}^{3-B C}: & : \tau_{j k}-\tau_{j k^{\prime}}-\tau_{j^{\prime} k}+\tau_{j^{\prime} k^{-}}+\tilde{\rho}_{. j k}-\tilde{\rho}_{. j^{-} k}-\tilde{\rho}_{. j k^{-}}+\tilde{\rho}_{. j^{\prime} k^{-}}=0 \\
& \forall\left(j, j^{\prime}, k, k^{\prime}\right), j \neq j^{\prime}, k \neq k^{\prime}
\end{aligned}
\end{gathered}
$$

vi Hipóteses para a interação $A * B * C$

$$
\begin{aligned}
H_{o}^{1-A B C}=H_{o}^{2-A B C}=H_{o}^{3-A B C}=H_{o}^{4-A B C} & : \rho_{i j k^{+}} \rho_{i j k^{-}}-\rho_{i j^{\prime} k}-\rho_{i j^{\prime} k^{-}}-\rho_{i^{\prime} j k}-\rho_{i^{\prime} j k^{-}}+\rho_{i^{-} j^{\prime} k^{+}}+\rho_{i^{\prime} j^{-} k^{-}}=0 \\
& \forall\left(i, i^{\prime} j, j^{\prime}, k, k^{\prime}\right), i \neq i^{\prime}, j \neq j^{\prime}, k \neq k^{\prime}
\end{aligned}
$$

Como pode ser observado, as hipóteses sobre a interação de mais alta ordem é a mesma para os quatro tipos e não contêm componentes das interações de ordem inferior.

\section{a Conjuntos balanceados}

\subsubsection{3o Exemplo}

Para os delineamentos ortogonais balanceados com três fatores, assim como feito para os modelos com dois fatores, são apresentados apenas alguns resultados quanto a igualdade de hipóteses e a forma das funções estumáveis, que serviram como uma referência para a discussão dos delineamentos desbalanceados com freqüências proporcionais nas caselas. 
Tabela 8. Dados de porcentagem de germinação de canafistula (Peltophorum dubium Spreng) do delineamento ortogonal balanceado com três fatores.

\begin{tabular}{|c|c|c|c|c|}
\hline \multirow[b]{4}{*}{ Temperatura (fator $A$ ) } & \multicolumn{4}{|c|}{ Dormência ( fator $C$ ) } \\
\hline & \multicolumn{2}{|c|}{$k=1$} & \multicolumn{2}{|c|}{$k=2$} \\
\hline & \multicolumn{2}{|c|}{ Substrato ( fator $B$ ) } & \multicolumn{2}{|c|}{ Substrato (fator $B$ ) } \\
\hline & $\dot{F}=1$ & $\dot{y}=2$ & $\dot{F}=1$ & $\dot{F}=2$ \\
\hline \multirow[t]{3}{*}{$F=1$} & 12 & 38 & 30 & 12 \\
\hline & 22 & 46 & 22 & 15 \\
\hline & 30 & 42 & 28 & 26 \\
\hline \multirow[t]{3}{*}{$I=2$} & 58 & 40 & 34 & 28 \\
\hline & 56 & 46 & 26 & 20 \\
\hline & 62 & 36 & 22 & 22 \\
\hline
\end{tabular}

\section{a.1 Modelo sem interações}

O Quadro 10 apresenta as funçōes estimáveis apenas para o fator $A$, mostrando que nos conjuntos balanceados, funçōes do próprio fator são estimáveis nos modelos sem interaçōes. Esse resultado também ocorre para as funções estimáveis relativas aos fatores $B$ e $C$, como pode ser verificado invertendo-se a ordem de entrada dos fatores no modelo.

Quadro 10. Funções estimáveis fornecidas pelo Proc GLM do SAS e respectivas hipóteses sobre o fator $A$, para os dados da Tabela 8 , considerando o modelo sem interações.

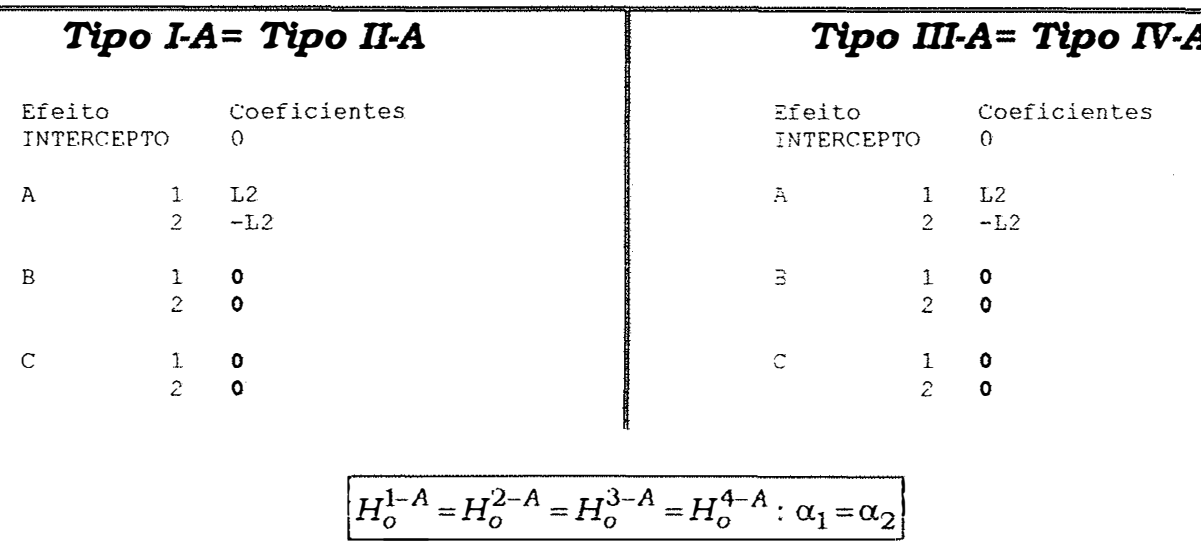

$\mathrm{Na}$ Tabela 9, estão destacados apenas os resultados para o fator A. A igualdade entre as somas de quadrados associadas às hipóteses dos tipos 
I e II, ou seja, $R(\alpha \mid \mu)=R(\alpha \mid \mu, \beta, \gamma)=672,0417$ que são, ainda, iguais às decomposições, $R(\alpha \mid \mu, \beta)$ e $R(\alpha \mid \mu, \gamma)$. As igualdades para as hipóteses dos efeitos principais e interações são apresentadas no conjunto desbalanceado. A igualdade entre as somas de quadrados associadas às hipóteses dos tipos II e III è devida à ausência de interaçōes, $R(\alpha \mid \mu, \beta, \gamma)=R(\dot{\alpha} \mid \dot{\mu}, \dot{\beta}, \dot{\gamma})=672,0147$,

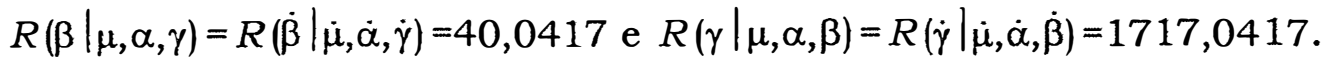

Tabela 9. Análise de variância do delineamento ortogonal balanceado, descrito na Tabela 8, segundo o modelo $y_{i j k l}=\mu+\alpha_{i}+\beta_{j}+\gamma_{k}+e_{i j k l}$, declarando A como primeiro fator.

\begin{tabular}{lcrrl}
\hline Fontes & gl & S.Q. Tipo I & Q.M. & $R(\cdot)$ \\
\hline A & 1 & 672,04166667 & 672,04166667 & $\boldsymbol{R}(\alpha \mid \mu)$ \\
B & 1 & 40,04166667 & 40,04166667 & $R(\beta \mid \mu, \alpha)$ \\
C & 1 & 1717,04166667 & 1717,04166667 & $R(\gamma \mid \mu, \alpha, \beta)$ \\
\hline Fontes & $g l$ & S.Q. Tipo II & $Q . M$. & $R(\cdot)$ \\
\hline A & 1 & 672,04166667 & 672,04166667 & $R(\alpha \mid \mu, \beta, \gamma)$ \\
B & 1 & 40,04166667 & 40,04166667 & $R(\beta \mid \mu, \alpha, \gamma)$ \\
C & 1 & 1717,04166667 & 1717,04166667 & $R(\gamma \mid \mu, \alpha, \beta)$ \\
\hline Fontes & $g l$ & SQ Tipo III=IV & $Q . M$. & $R(\cdot)$ \\
\hline A & 1 & 672,04166667 & 672,04166667 & $R(\dot{\alpha} \mid \dot{\mu}, \dot{\beta}, \dot{\gamma})$ \\
B & 1 & 40,04166667 & 40,04166667 & $R(\dot{\beta} \mid \dot{\mu}, \dot{\alpha}, \dot{\gamma})$ \\
C & 1 & 1717,04166667 & 1717,04166667 & $R(\dot{\gamma} \mid \dot{\mu}, \dot{\alpha}, \dot{\beta})$ \\
\hline
\end{tabular}

\section{a.2 Modelo com interações}

Pelo Quadro 11 observa-se a caracteristica dos delineamentos ortogonais balanceados de modelos com três fatores e interações, onde as funções estimáveis envolvendo um único fator, no exemplo, o fator $A$, apresentaram parâmetros relativos ao próprio fator $(\alpha)$ e parâmetros das interações onde o fator está presente ( $\varphi$ e $\rho)$. Ademais, funções estimáveis das interações, no exemplo, a interação $A^{*} B$ como se observa no Quadro 12, revelam a presença de parâmetros da própria interação $(\varphi)$ e parâmetros da interação de maior grau ( $\rho)$. 
Quadro 11. Funções estimáveis fornecidas pelo Proc GLM do SAS e respectivas hipóteses sobre o fator $A$, para os dados da Tabela 8 , considerando o modelo com interações.

\begin{tabular}{|c|c|c|c|c|c|}
\hline \multicolumn{3}{|c|}{ Tipo I-A = Tipo II-A } & \multicolumn{3}{|c|}{ Tipo III-A = Tipo IV-A } \\
\hline \multicolumn{2}{|c|}{$\begin{array}{l}\text { Efeito } \\
\text { INTERCEPTO }\end{array}$} & $\begin{array}{l}\text { Coeficientes } \\
0\end{array}$ & \multicolumn{2}{|c|}{$\begin{array}{l}\text { Efeito } \\
\text { INTERCEPTO }\end{array}$} & \multirow{2}{*}{$\begin{array}{l}\text { Coeficientes } \\
0 \\
\text { L2 } \\
-\mathrm{L} 2\end{array}$} \\
\hline A & $\begin{array}{l}1 \\
2\end{array}$ & $\begin{array}{l}\mathrm{L} 2 \\
-\mathrm{L} 2\end{array}$ & A & $\begin{array}{l}1 \\
2\end{array}$ & \\
\hline $\mathbf{2}$ & $\begin{array}{l}1 \\
2\end{array}$ & $\begin{array}{l}0 \\
0\end{array}$ & B & $\begin{array}{l}1 \\
2\end{array}$ & $\begin{array}{l}0 \\
0\end{array}$ \\
\hline C & $\begin{array}{l}1 \\
2\end{array}$ & $\begin{array}{l}0 \\
0\end{array}$ & c & $\begin{array}{l}1 \\
2\end{array}$ & $\begin{array}{l}0 \\
0\end{array}$ \\
\hline$A^{\star} B$ & $\begin{array}{ll}1 & 1 \\
1 & 2 \\
2 & 1 \\
2 & 2\end{array}$ & $\begin{array}{l}0,5 * L 2 \\
\hat{U}, 5 * \mathrm{~L} 2 \\
-0,5 * \mathrm{~L} 2 \\
-0,5 * \mathrm{~L} 2\end{array}$ & $A * B$ & $\begin{array}{ll}1 & 1 \\
1 & 2 \\
2 & 1 \\
2 & 2\end{array}$ & $\begin{array}{l}0,5 * \mathrm{~L} 2 \\
0,5 * \mathrm{~L} 2 \\
-0,5 * \mathrm{~L} 2 \\
-0,5 * \mathrm{~L} 2\end{array}$ \\
\hline$A * C$ & $\begin{array}{ll}1 & 1 \\
1 & 2 \\
2 & 1 \\
2 & 2\end{array}$ & $\begin{array}{l}0,5 * \mathrm{~L} 2 \\
0,5 * \mathrm{~L} 2 \\
-0,5 * \mathrm{~L} 2 \\
-0,5 * \mathrm{~L} .2\end{array}$ & $A^{\star}{ }^{*} C$ & $\begin{array}{ll}1 & 1 \\
1 & 2 \\
2 & 1 \\
2 & 2\end{array}$ & $\begin{array}{l}0,5 * \mathrm{~L} 2 \\
0,5 * \mathrm{~L} 2 \\
-0,5 * \mathrm{~L} 2 \\
-0,5 * \mathrm{I} 2\end{array}$ \\
\hline $\mathrm{B}^{\star} \mathrm{C}$ & $\begin{array}{ll}1 & 1 \\
1 & 2 \\
2 & 1 \\
2 & 2\end{array}$ & $\begin{array}{l}0 \\
0 \\
0 \\
0\end{array}$ & $B^{\star} C$ & $\begin{array}{ll}1 & 1 \\
1 & 2 \\
2 & 1 \\
2 & 2\end{array}$ & $\begin{array}{l}0 \\
0 \\
0 \\
0\end{array}$ \\
\hline$A * B * C$ & $\begin{array}{lll}1 & 1 & 1 \\
1 & 1 & 2 \\
1 & 2 & 1 \\
1 & 2 & 2 \\
2 & 1 & 1 \\
2 & 1 & 2 \\
2 & 2 & 1 \\
2 & 2 & 2\end{array}$ & $\begin{array}{l}0,25 * \mathrm{~L} 2 \\
0,25 * \mathrm{~L} 2 \\
0,25 * \mathrm{~L} 2 \\
0,25 * \mathrm{~L} 2 \\
-0,25 * \mathrm{~L} 2 \\
-0,25 * \mathrm{~L} 2 \\
-0,25 * \mathrm{~L} 2 \\
-0,25 * \mathrm{~L} 2\end{array}$ & $A * B * C$ & $\begin{array}{lll}1 & 1 & 1 \\
1 & 1 & 2 \\
1 & 2 & 1 \\
1 & 2 & 2 \\
2 & 1 & 1 \\
2 & 1 & 2 \\
2 & 2 & 1 \\
2 & 2 & 2\end{array}$ & $\begin{array}{l}0,25 * \mathrm{~L} 2 \\
0,25 * \mathrm{~L} 2 \\
0,25 * \mathrm{~L} 2 \\
0,25 * \mathrm{~L} 2 \\
-0,25 * \mathrm{~L} 2 \\
-0,25 * \mathrm{~L} 2 \\
-0,25 * \mathrm{~L} 2 \\
-0,25 * \mathrm{~L} 2\end{array}$ \\
\hline$H_{O}^{1-A}=F$ & $A=H_{O}^{3}$ & $\begin{array}{r}=H_{o}^{4-A}: \alpha_{1}+ \\
=\alpha_{2}\end{array}$ & $\begin{array}{l}2 \eta_{12}+p \\
+\eta_{22}+p\end{array}$ & $\begin{array}{l}+\rho_{112} \\
+\rho_{212}\end{array}$ & $\begin{array}{l}\left.121+\rho_{122}\right)= \\
\left.p_{221}+\rho_{222}\right)\end{array}$ \\
\hline
\end{tabular}


Quadro 12. Funções estimáveis fornecidas pelo Proc GLM do SAS e respectivas hipóteses sobre a interação fator $A^{*} B$, para os dados da Tabela 8.

\begin{tabular}{|c|c|c|c|c|c|}
\hline \multicolumn{3}{|c|}{ Tlpo I-A*B $=$ Tipo II-A*B } & \multicolumn{3}{|c|}{ Tipo III-A*B $=I V-A * B$} \\
\hline \multicolumn{2}{|c|}{$\begin{array}{l}\text { Efeito } \\
\text { INTERCEPTO }\end{array}$} & \multirow{2}{*}{$\begin{array}{l}\text { Coeficientes } \\
0 \\
0 \\
0\end{array}$} & \multicolumn{2}{|c|}{$\begin{array}{l}\text { Ex́feito } \\
\text { INTERCEPTO }\end{array}$} & \multirow{2}{*}{$\begin{array}{l}\text { Coeficientes } \\
0 \\
0 \\
0\end{array}$} \\
\hline A & $\begin{array}{l}1 \\
2\end{array}$ & & A & $\begin{array}{l}1 \\
2\end{array}$ & \\
\hline B & $\begin{array}{l}1 \\
2\end{array}$ & $\begin{array}{l}0 \\
0\end{array}$ & B & $\begin{array}{l}1 \\
2\end{array}$ & $\begin{array}{l}0 \\
0\end{array}$ \\
\hline c & $\begin{array}{l}1 \\
2\end{array}$ & 0 & c & $\begin{array}{l}1 \\
2\end{array}$ & $\begin{array}{l}0 \\
0\end{array}$ \\
\hline$A^{\star} B$ & $\begin{array}{ll}1 & 1 \\
1 & 2 \\
2 & 1 \\
2 & 2\end{array}$ & $\begin{array}{l}\mathrm{L} 8 \\
-\mathrm{L} 8 \\
-\mathrm{L} 8 \\
\mathrm{~L} 8\end{array}$ & $A^{* B}$ & $\begin{array}{ll}1 & 1 \\
1 & 2 \\
2 & 1 \\
2 & 2\end{array}$ & $\begin{array}{l}\mathrm{L} 8 \\
-\mathrm{L} 6 \\
-\mathrm{L} 8 \\
\mathrm{~L} 8\end{array}$ \\
\hline$A \star C$ & $\begin{array}{ll}1 & 1 \\
1 & 2 \\
2 & 1 \\
2 & 2\end{array}$ & $\begin{array}{l}0 \\
0 \\
0 \\
0\end{array}$ & $A * C$ & $\begin{array}{ll}1 & 1 \\
1 & 2 \\
2 & 1 \\
2 & 2\end{array}$ & $\begin{array}{l}0 \\
0 \\
0 \\
0\end{array}$ \\
\hline$B * C$ & $\begin{array}{ll}1 & 1 \\
1 & 2 \\
2 & 1 \\
2 & 2\end{array}$ & $\begin{array}{l}0 \\
0 \\
0 \\
0\end{array}$ & $\mathrm{~B}^{*} \mathrm{C}$ & $\begin{array}{ll}1 & 1 \\
1 & 2 \\
2 & 1 \\
2 & 2\end{array}$ & $\begin{array}{l}0 \\
0 \\
0 \\
0\end{array}$ \\
\hline$A * B * C$ & $\begin{array}{lll}1 & 1 & 1 \\
1 & 1 & 2 \\
1 & 2 & 1 \\
1 & 2 & 2 \\
2 & 1 & 1 \\
2 & 1 & 2 \\
2 & 2 & 1 \\
2 & 2 & 2\end{array}$ & 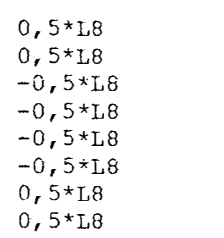 & $A * B * C$ & $\begin{array}{lll}1 & 1 & 1 \\
1 & 1 & 2 \\
1 & 2 & 1 \\
1 & 2 & 2 \\
2 & 1 & 1 \\
2 & 1 & 2 \\
2 & 2 & 1 \\
2 & 2 & 2\end{array}$ & $\begin{array}{l}0,5 * \mathrm{~L} 8 \\
0,5 * \mathrm{~L} 8 \\
-0,5 * \mathrm{~L} \theta \\
-0,5 * \mathrm{~L} 8 \\
-0,5 * \mathrm{~L} 8 \\
-0,5 * \mathrm{~L} 8 \\
0,5 * \mathrm{~L} 8 \\
0,5 * \mathrm{~L} 8\end{array}$ \\
\hline$H_{o}^{1-A B}=H_{o}^{2}$ & $=H_{0}^{3-t}$ & $\begin{aligned} 3=H_{O}^{4-A B}: & \varphi_{11} \\
& +\rho_{22}\end{aligned}$ & ${ }_{2}\left(\rho_{11}\right.$ & $12-\rho_{1}$ & $-\rho_{122}-\rho_{211}$ \\
\hline
\end{tabular}

Na Tabela 10 observa-se, quanto aos efeitos principais de modelos com interações, a mesma igualdade de hipóteses e decomposições verificadas nos modelos sem interações, ou seja, $R(\alpha \mid \mu)=R(\alpha \mid \mu, \beta, \gamma)=672,0417$. As somas de quadrados das interações, no exemplo, a interação $A * B$, mostram a igualdade entre $R(\varphi \mid \mu, \alpha, \beta, \gamma)=R(\varphi \mid \mu, \alpha, \beta, \gamma, \eta, \tau)=425,0417$, ou seja, a soma de quadrados associada à hipótese do tipo I para a interação. $A * B$ é igual a uma soma de quadrados associada à hipótese do tipo II. Esses resultados só puderam ser ilustrados para o fator $A$ e para a interação $A^{*} B$, uma vez que entre os efeitos principais e entre as interações foram os primeiros a serem 
declarados. Maiores detalhes quanto a ordem de entrada dos efeitos principais e das interações são apresentados no conjunto desbalanceado com freqüências proporcionais.

Tabela 10. Análise de variância do delineamento ortogonal balanceado, descrito na Tabela 8, segundo o modelo $y_{i j k l}=\mu+\alpha_{i}+\beta_{j}+\gamma_{k}+\varphi_{i j}+\eta_{i k}+\tau_{j k}+\rho_{i j k}+e_{i j k l}$, sendo $A$ o primeiro fator declarado entre os efeitos principais e $A * B$ a primeira entre as interações.

\begin{tabular}{|c|c|c|c|c|}
\hline Fontes & $g l$ & S.Q. Tipo I & Q.M. & $R()$. \\
\hline A & 1 & 672,04166667 & 672,04166667 & $R(\alpha \mid \mu)$ \\
\hline B & 1 & 40,04166667 & 40,04166667 & $R(\beta \mid \mu, \alpha)$ \\
\hline C & 1 & 1717,04166667 & 1717,04166667 & $R(\gamma \mid \mu, \alpha, \beta)$ \\
\hline$A * B$ & 1 & 425,04166667 & 425,04166667 & $R(\Phi \mid \mu, \alpha, \beta, \gamma)$ \\
\hline$A^{\star} \mathrm{C}$ & 1 & 330,04166667 & 330,04166667 & $R(\eta \mid \mu, \alpha, \beta, \gamma, \varphi)$ \\
\hline$B{ }^{*} \mathrm{C}$ & 1 & 92,04166667 & 92,04166667 & $R(\tau \mid \mu, \alpha, \beta, \gamma, \varphi, \eta)$ \\
\hline$A * B * C$ & 1 & 715,04166667 & 715,04166667 & $R(\rho \mid \mu, \alpha, \beta, \gamma, \varphi, \eta, \tau)$ \\
\hline Fontes & $g l$ & S.Q. Tipo II & Q.M. & $R()$. \\
\hline A & 1 & 672,04166667 & 672,04166667 & $R(\alpha \mid \mu, \beta, \gamma)$ \\
\hline B & 1 & $40,0416666 \%$ & $40,0416666 \%$ & $R(\beta \mid \mu, \alpha, \gamma)$ \\
\hline c & 1 & 1717,04166667 & 1717,04166667 & $R(\gamma \mid \mu, \alpha, \beta)$ \\
\hline$A^{* B}$ & 1 & 425,04166667 & 425,04166667 & $\boldsymbol{R}(\varphi \mid \mu, \alpha, \beta, \gamma, \eta, \tau)$ \\
\hline$A^{*} \mathrm{C}$ & 1 & 330,04166667 & 330,04166667 & $R(\eta \mid \mu, \alpha, \beta, \gamma, \varphi, \tau)$ \\
\hline$B * C$ & 1 & 92,04166667 & 92,04166667 & $R(\tau \mid \mu, \alpha, \beta, \gamma, \varphi, \eta)$ \\
\hline$A{ }^{\star} B{ }^{\star} C$ & 1 & 715,04166667 & 715,04166667 & $R(\rho \mid \mu, \alpha, \beta, \gamma, \varphi, \eta, \tau)$ \\
\hline Fontes & $g l$ & SQ Tipo III $=I V$ & Q.M. & $R()$. \\
\hline A & 1 & 672,04166667 & 672,04166667 & $R(\dot{\alpha} \mid \dot{\mu}, \dot{\beta}, \dot{\gamma})$ \\
\hline$B$ & 1 & 40,04166667 & 40,04166667 & $R(\dot{\beta} \mid \dot{\mu}, \dot{\alpha}, \dot{\gamma})$ \\
\hline c & 1 & 1717,04166667 & 1717,04166667 & $R(\dot{\gamma} \mid \dot{\mu}, \dot{\alpha}, \dot{\beta})$ \\
\hline$A * B$ & 1 & 425,04166667 & 425,04166667 & $\boldsymbol{R}(\dot{\boldsymbol{\phi}} \mid \dot{\mu}, \dot{\alpha}, \dot{\beta}, \dot{\gamma}, \dot{\eta}, \dot{\tau})$ \\
\hline$A^{*} \mathrm{C}$ & 1 & 330,04166667 & 330,04166667 & $R(\dot{\eta} \mid \dot{\mu}, \dot{\alpha}, \dot{\beta}, \dot{\gamma}, \dot{\varphi}, \dot{\tau})$ \\
\hline$B{ }^{\star} \mathrm{C}$ & 1 & 92,04166667 & 92,04166667 & $R(\dot{\tau} \mid \dot{\mu}, \dot{\alpha}, \dot{\beta}, \dot{\gamma}, \dot{\varphi}, \dot{\eta})$ \\
\hline$A \star B \star C$ & 1 & 715,04166667 & 715,04166667 & $R(\dot{\rho} \mid \dot{\mu}, \dot{\alpha}, \dot{\beta}, \dot{\gamma}, \dot{\varphi}, \dot{\eta}, \dot{\tau})$ \\
\hline
\end{tabular}

Nos modelos com três fatores e interações, é importante nāo só a ordem de entrada dos efeitos principais, mas também, a ordem de entrada das interações. A variação na ordem de entrada das interações, amplia o estudo da ortogonalidade, antes centrada na igualdade entre as hipóteses dos tipos I e II para efeitos principais, agora expandida para as interações. 


\section{b Conjuntos desbalanceados}

\subsubsection{4o Exemplo}

A Tabela 11 apresenta o exemplo do delineamento ortogonal desbalanceado com três fatores, através do qual são feitas discussões sobre igualdade de hipóteses, formas das funções estimáveis e formas de obtenção das hipóteses dos tipos I e II para os efeitos principais e interações pelo Proc GLM do SAS. Na direçāo dos delineamentos ortogonais desbalanceados com mais de dois fatores é que se concentrou grande parte dos objetivos deste wabalho e por isso a ortogonalidade foi abordada com maior detalhamento.

Tabela 11. Dados de porcentagem de germinação de canafistula (Peltophorum dubium Spreng) do delineamento ortogonal desbalanceado com três fatores.

\begin{tabular}{|c|c|c|c|c|}
\hline \multirow[b]{4}{*}{ Temperatura (fator $A$ ) } & \multicolumn{4}{|c|}{ Substrato ( fator $B$ ) } \\
\hline & \multicolumn{2}{|c|}{$j=1$} & \multicolumn{2}{|c|}{$\dot{j}=2$} \\
\hline & \multicolumn{2}{|c|}{ Dormência ( fator $C$ ) } & \multicolumn{2}{|c|}{ Dormência ( fator $C$ ) } \\
\hline & $k=1$ & $k=2$ & $k=1$ & $k=2$ \\
\hline \multirow[t]{3}{*}{$i=1$} & 12 & 28 & 38 & 40 \\
\hline & & & 46 & 46 \\
\hline & & & 42 & 36 \\
\hline \multirow[t]{6}{*}{$i=2$} & 30 & 34 & 12 & 58 \\
\hline & 22 & 26 & 22 & 56 \\
\hline & & & 30 & 62 \\
\hline & & & 12 & 52 \\
\hline & & & 18 & 58 \\
\hline & & & 38 & 70 \\
\hline
\end{tabular}

No teorema 2 comprovou-se, que uma condiçāo necessária e suficiente para que os subespaços gerados pelas colunas das submatrizes de $X$ referentes a cada fator, após o ajuste de $\mu$, sejam ortogonais e portanto, que um delineamento com três fatores seja ortogonal, é que as freqüências das caselas dos fatores dois a dois sejam proporcionais, sendo tais proporções apresentadas nas expressões 10, 15 e 20.

Aplicando-se as expressões aos dados da Tabela 11, foram obtidas as seguintes proporções: 
i) Ignorando o fator C

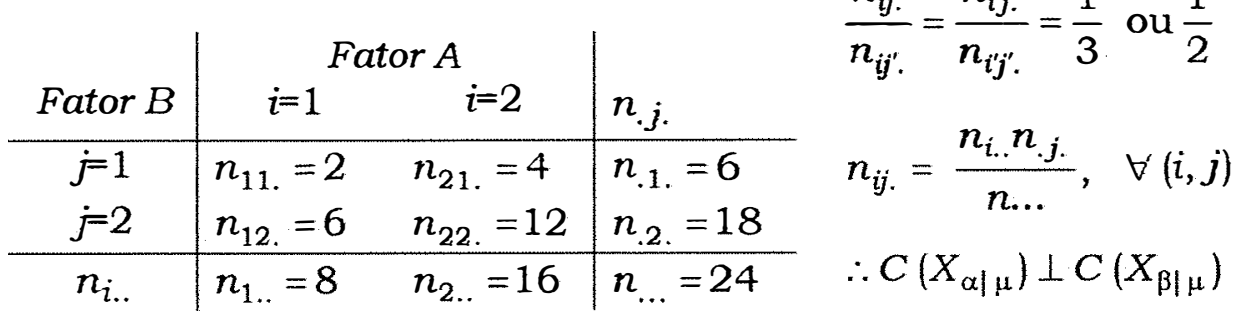

\section{ii) Ignorando o fator $B$}

\begin{tabular}{|c|c|c|c|c|}
\hline \multirow[b]{2}{*}{ Fator C } & \multicolumn{2}{|c|}{ Fator A } & \multirow[b]{2}{*}{$n$} & \multirow[t]{2}{*}{$\frac{n_{i . k}}{n_{i^{\prime} . k}}=\frac{n_{i . k^{\prime}}}{n_{i^{\prime} \cdot k^{\prime}}}=\frac{1}{2}$ ou 1} \\
\hline & $i=1$ & $i=2$ & & \\
\hline$k=1$ & $n_{11}=4$ & $n_{21}=8$ & $n_{1}=12$ & $\underline{n_{i . .} n_{. . k}}$ \\
\hline$k=2$ & $n_{1.2}=4$ & $n_{2.2}=8$ & $n_{. .2}=12$ & \\
\hline$n_{i . .}$ & $n_{1 . .}=8$ & $n_{2 . .}=16$ & $n_{\ldots}=24$ & $\therefore C\left(X_{\alpha \mid \mu}\right) \perp$ \\
\hline
\end{tabular}

iii) Ignorando o fator $A$

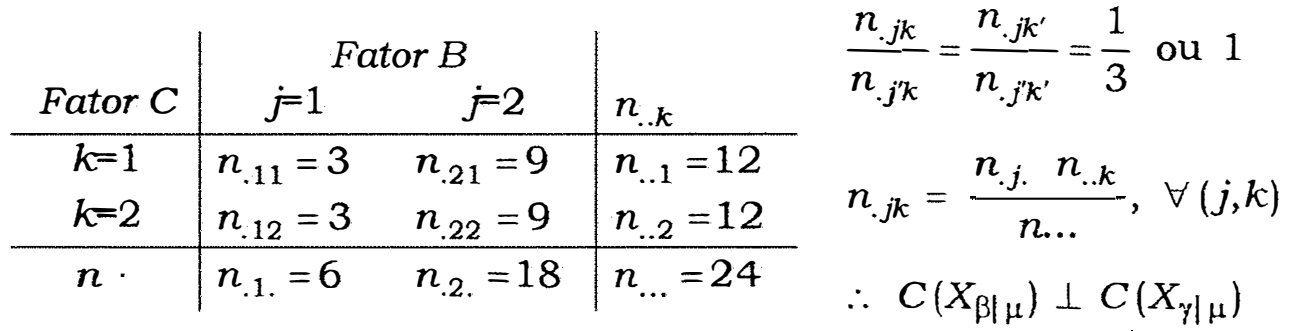


Como as freqüèncias entre os fatores dois a dois sāo proporcionais, o delineamento desbalanceado apresentado na Tabela 11 é ortogonal e portanto as hipóteses sobre médias ponderadas são iguais às hipóteses sobre médias ponderadas ajustadas, para todos os efeitos principais e interações. Essa afirmação e outras consideraçōes sobre a ortogonalidade em conjuntos desbalanceados sāo apresentados à seguir dentro de modelos com e sem interações.

\section{b. 1 Modelos sem interações}

A forma matricial do modelo $y=X \theta+e$ caracterizado por $y_{i j k l}=\mu+\alpha_{i}+\beta_{j}+\gamma_{k}+e_{i j k l}$, para os dados da Tabela 11 é dada por:

$$
\left[\begin{array}{l}
12 \\
28 \\
38 \\
46 \\
42 \\
40 \\
46 \\
36 \\
30 \\
22 \\
34 \\
26 \\
12 \\
22 \\
30 \\
12 \\
18 \\
38 \\
1 \\
58 \\
56 \\
1 \\
62 \\
1
\end{array}\right]=\left[\begin{array}{l:ll:ll:ll:llll}
1 & 1 & 1 & 0 & 0 & 0 & 1 & 1 & 1 & 0 \\
1 & 1 & 0 & 0 & 1 & 0 & 1 \\
1 & 1 & 0 & 0 & 1 & 0 & 1 \\
1 & 1 & 0 & 0 & 1 & 0 & 1 \\
1 & 0 & 1 & 1 & 0 & 1 & 0 \\
1 & 0 & 1 & 1 & 0 & 1 & 0 \\
1 & 0 & 1 & 1 & 0 & 0 & 1 \\
1 & 0 & 1 & 1 & 0 & 0 & 1 \\
1 & 0 & 1 & 0 & 1 & 1 & 0 \\
1 & 0 & 1 & 0 & 1 & 1 & 0 \\
1 & 0 & 1 & 0 & 1 & 1 & 0 \\
1 & 0 & 1 & 0 & 1 & 1 & 0 \\
1 & 0 & 1 & 0 & 1 & 1 & 0 \\
1 & 0 & 1 & 0 & 1 & 1 & 0 \\
1 & 0 & 1 & 0 & 1 & 0 & 1 \\
1 & 0 & 1 & 0 & 1 & 0 & 1 \\
1 & 0 & 1 & 0 & 1 & 0 & 1 \\
1 & 0 & 1 & 0 & 1 & 0 & 1 \\
1 & 0 & 1 & 0 & 1 & 0 & 1 \\
1 & 0 & 1 & 0 & 1 & 0 & 1
\end{array}\right]\left[\begin{array}{l}
\mu \\
\hdashline \alpha_{1} \\
\alpha_{2} \\
\beta_{1} \\
\beta_{2} \\
\gamma_{1} \\
\gamma_{2}
\end{array}\right]+\left[\begin{array}{l}
e_{111} \\
e_{1121} \\
e_{1211} \\
e_{1212} \\
e_{1213} \\
e_{1221} \\
e_{1222} \\
e_{1223} \\
e_{2111} \\
e_{2112} \\
e_{2121} \\
e_{2122} \\
e_{2211} \\
e_{2212} \\
e_{2213} \\
e_{2214} \\
e_{2215} \\
e_{2216} \\
e_{2221} \\
e_{2222} \\
e_{2223} \\
e_{2224} \\
e_{2225} \\
e_{2225}
\end{array}\right]
$$

onde:

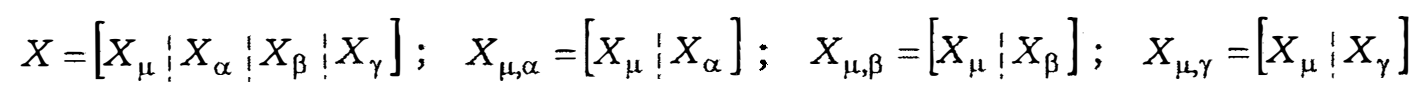


Para ilustrar as propriedades dos delineamentos ortogonais desbalanceados com três fatores, foram utilizados os dados da Tabela $11 \mathrm{e}$ formuladas hipóteses dos tipos I e II apenas para o fator $A$, segundo o critério de Searle (1987). O modelo adotado para a construção das hipóteses foi o de médias de caselas caracterizado por $y_{i j k l}=\mu_{i}+\beta_{j}+\gamma_{k}+e_{i j k l}$ (Speed, Hocking e Hackney, 1978), como apresenta a Tabela 12.

Tabela 12. Representação do modelo de médias caselas do delineamento ortogonal desbalanceado descrito na Tabela 11.

\begin{tabular}{|c|c|c|c|c|}
\hline \multirow[b]{4}{*}{ Fator A } & \multicolumn{4}{|c|}{ Fator B } \\
\hline & \multicolumn{2}{|c|}{$j=1$} & \multicolumn{2}{|c|}{$j=2$} \\
\hline & \multicolumn{2}{|c|}{ Fator $C$} & \multicolumn{2}{|c|}{ Fator C } \\
\hline & $k=1$ & $k=2$ & $k=1$ & $k=2$ \\
\hline$i=1$ & $\mu_{1}+\beta_{1}+\gamma_{1}^{(1)}$ & $\mu_{1}+\beta_{1}+\gamma_{2}^{(1)}$ & $\mu_{1}+\beta_{2}+\gamma_{1}^{(3)}$ & $\mu_{1}+\beta_{2}+\gamma_{2}{ }^{(3)}$ \\
\hline$i=2$ & $\mu_{2}+\beta_{1}+\gamma_{1}^{(2)}$ & $\mu_{2}+\beta_{1}+\gamma_{2}^{(2)}$ & $\mu_{2}+\beta_{2}+\gamma_{1}^{(6)}$ & $\mu_{2}+\beta_{2}+\gamma_{2}{ }^{(\sigma)}$ \\
\hline
\end{tabular}

\section{a Hipótese do tipo I para of fator $A$}

Observa-se, sob ortogonalidade, que através da ponderação pelas freqüências de caselas da hipótese do tipo I para o fator $A$, já ocorre automaticamente o ajuste para os outros fatores.

$$
\begin{aligned}
H_{o}^{1-A} & : \frac{\left(\mu_{1}+\beta_{1}+\gamma_{1}\right)+\left(\mu_{1}+\beta_{1}+\gamma_{2}\right)+3\left(\mu_{1}+\beta_{2}+\gamma_{1}\right)+3\left(\mu_{1}+\beta_{2}+\gamma_{2}\right)}{8}= \\
& =\frac{2\left(\mu_{2}+\beta_{1}+\gamma_{1}\right)+2\left(\mu_{2}+\beta_{1}+\gamma_{2}\right)+6\left(\mu_{2}+\beta_{2}+\gamma_{1}\right)+6\left(\mu_{2}+\beta_{2}+\gamma_{2}\right)}{16}
\end{aligned}
$$

Uma simplificação é apresentada no modelo-M e posteriormente transformada no modelo-S.

\section{a. 1 Modelo-M}

$H_{o}^{1-A}: \mu_{1}=\mu_{2}$

\section{a.2 Modelo-S}

$$
H_{o}^{1-A}: \alpha_{1}=\alpha_{2}
$$




\section{b Hipótese do tipo II para o fator A}

São as hipóteses do tipo II para conjuntos completos, as mais importantes para a interpretação prática de resultados. Mas, no exemplo a ortogonalidade faz com que os tipos I e II sejam equivalentes o que valoriza e estimula o planejamento de delineamentos ortogonais.

$H_{o}^{2-A}:-\frac{\left(\mu_{1}+\beta_{1}+\gamma_{1}\right)+\left(\mu_{1}+\beta_{1}+\gamma_{2}\right)+3\left(\mu_{1}+\beta_{2}+\gamma_{1}\right)+3\left(\mu_{1}+\beta_{2}+\gamma_{2}\right)}{8}=\frac{\bar{C}_{1}+\bar{C}_{2}+3 \bar{C}_{3}+3 \bar{C}_{4}}{8}$

onde:

$$
\begin{array}{ll}
\bar{C}_{1}=\frac{\left(\mu_{1}+\beta_{1}+\gamma_{1}\right)+2\left(\mu_{2}+\beta_{1}+\gamma_{1}\right)}{3} ; & \bar{C}_{3}=\frac{3\left(\mu_{1}+\beta_{2}+\gamma_{1}\right)+6\left(\mu_{2}+\beta_{2}+\gamma_{1}\right)}{9} \\
\bar{C}_{2}=\frac{\left(\mu_{1}+\beta_{1}+\gamma_{2}\right)+2\left(\mu_{2}+\beta_{1}+\gamma_{2}\right)}{3} ; & \bar{C}_{4}=\frac{3\left(\mu_{1}+\beta_{2}+\gamma_{2}\right)+6\left(\mu_{2}+\beta_{2}+\gamma_{2}\right)}{9}
\end{array}
$$

\section{b. 1 Modelo-M}

$H_{o}^{1-A}: \mu_{1}=\mu_{2}$

\section{b.2 Modelo-S}

$$
H_{o}^{2-A}: \alpha_{1}=\alpha_{2}
$$

Observando-se o resultado do item a.2, pode-se verificar que nas funções estimáveis de delineamentos ortogonais com três fatores, funções do próprio parâmetro em estudo são estimáveis. No exemplo, o fator $A$. Consequentemente, ocorre a igualdade entre as funçōes estimáveis dos tipos. I e II como verificado nos itens a.2 e b.2. Esses resultados podem ser ainda ilustrados no Quadro 13. 
Quadro 13. Funções estimáveis fornecidas pelo Proc GLM do SAS e respectivas hipóteses sobre o fator $A$, para os dados da Tabela 11, considerando o modelo sem interaçōes.

\begin{tabular}{|c|c|c|c|c|c|}
\hline \multicolumn{3}{|c|}{ Tipo I-A = Tipo II-A } & \multicolumn{3}{|c|}{ Tipo II-A= Tipo IV-A } \\
\hline \multicolumn{2}{|c|}{$\begin{array}{l}\text { Efeito } \\
\text { INTERCEPTO }\end{array}$} & $\begin{array}{l}\text { Coeficientes } \\
0\end{array}$ & \multicolumn{2}{|c|}{$\begin{array}{l}\text { Efeito } \\
\text { INTERCEPTO }\end{array}$} & \multirow{2}{*}{$\begin{array}{l}\text { Coeficientes } \\
0 \\
\mathrm{~L} 2 \\
-\mathrm{L} 2\end{array}$} \\
\hline A & $\begin{array}{l}1 \\
2\end{array}$ & $\begin{array}{l}\mathrm{L} 2 \\
-\mathrm{L} 2\end{array}$ & $F$ & $\begin{array}{l}1 \\
2\end{array}$ & \\
\hline B & $\begin{array}{l}1 \\
2\end{array}$ & $\begin{array}{l}0 \\
0\end{array}$ & $E$ & $\begin{array}{l}1 \\
2\end{array}$ & $\begin{array}{l}0 \\
0\end{array}$ \\
\hline \multirow[t]{2}{*}{ c } & $\begin{array}{l}1 \\
2\end{array}$ & $\begin{array}{l}0 \\
0\end{array}$ & c & $\begin{array}{l}1 \\
2\end{array}$ & $\begin{array}{l}0 \\
0\end{array}$ \\
\hline & & $H_{o}^{1-A}=$ & & & \\
\hline
\end{tabular}

A Tabela 13 apresenta a análise da variância dos dados da Tabela 11, mostrando que para o fator $A$, a soma de quadrados associadas às hipóteses dos tipos I e II são iguais. Como conseqüência dessa igualdade, temse $R(\alpha \mid \mu)=R(\alpha \mid \mu, \beta, \gamma)=12,0000$; que por sua vez sāo iguais à $R(\alpha \mid \mu, \beta)$, apresentada na Tabela 14 e $R(\alpha \mid \mu, \gamma)$ apresentada na Tabela 15.

Para verificar a primeira igualdade, $R(\alpha \mid \mu)=R(\alpha \mid \mu, \beta, \gamma)$ foi aplicada a prova apresentada por Christensen (1987) que se baseia na igualdade entre os projetores ortogonais de cada decomposição. Aqui, para um modelo com três fatores sem interaçōes, a soma de quadrados de parâmetros, $R(\mu, \alpha, \beta, \gamma)$, tem o seguinte projetor ortogonal: $P=P_{\mu}+P_{\gamma \mid \mu}+P_{\beta \mid \mu, \gamma}+P_{\alpha \mid \mu, \beta, \gamma}$, que corresponde ao projetor da matriz de delineamento $X$. Se o projetor $P$ puder ser escrito como $P^{\prime}=P_{\mu}+P_{\gamma \mid \mu}+P_{\left.\beta\right|_{\mu, \gamma}}+P_{\alpha \mid \mu}$ implica que o subespaço gerado por $P^{\prime}$ é igual ao subespaço gerado pelas colunas de $X$ e, portanto, $P^{\prime}=P$.

Para o. exemplo da Tabela 11 , os vetores. linearmente independentes que formam uma das bases do subespaço gerado pelas colunas de $X$ e de $P^{\prime}=P_{\mu}+P_{\gamma \mid \mu}+P_{\left.\beta\right|_{\mu, \gamma}}+P_{\left.\alpha\right|_{\mu}}$, são apresentados a seguir. 

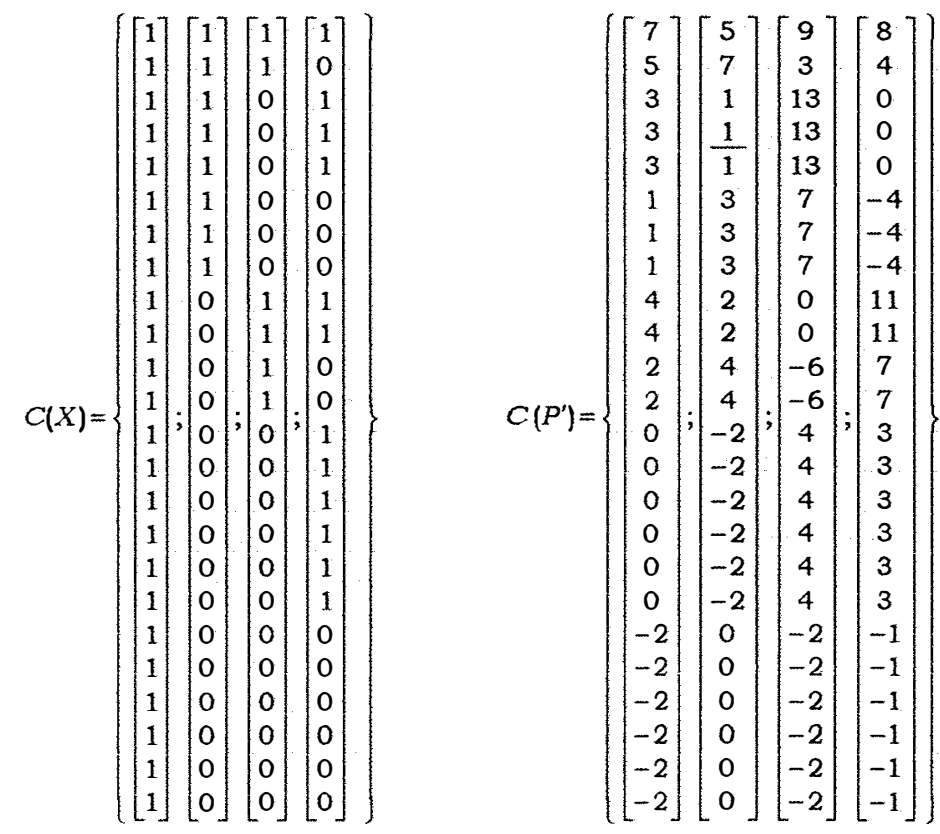

Como $C\left(P^{\prime}\right) \subset C(X)$, entāo $P^{\prime}=P$, Isso justifica o porquê de $R(\alpha \mid \mu)$ e $R(\alpha \mid \mu, \beta, \gamma)$ serem iguais, como pode ser observado na Tabela 15 . Como conseqüência, $P_{\alpha \mid \mu, \beta, \gamma}=P_{\alpha \mid \mu}=P_{\alpha \mid \mu, \beta}=P_{\alpha \mid \mu, \gamma}, \quad \operatorname{logo}, \quad R(\alpha \mid \mu)=R(\alpha \mid \mu, \beta)=$ $=R(\alpha \mid \mu, \gamma)=R(\alpha \mid \mu, \beta, \gamma)$. O projetor ajustado é apresentado a seguir.

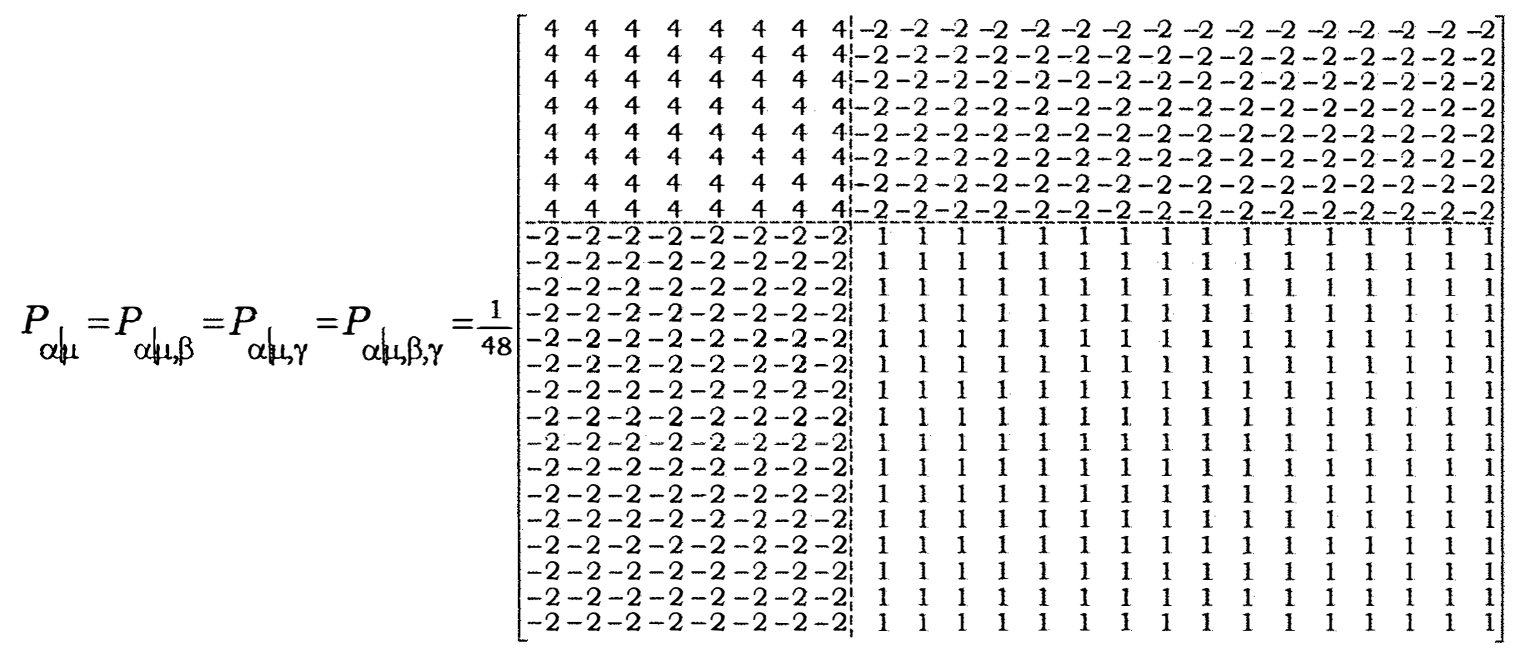


Entretanto, para que o delineamento seja definido como ortogonal é preciso também que o subespaço gerado pelas colunas de $P^{n}=P_{\mu}+P_{\alpha \mid \mu}+P_{\gamma \mid \mu, \alpha}+P_{\beta \mid \mu} \quad$ e $\quad$ o subespaço gerado pelas colunas $P^{m}=P_{\mu}+P_{\alpha \mid \mu}+P_{\beta \mid \mu, \gamma}+P_{\gamma \mid \mu}$ estejam individualmente contidos no subespaço gerado pelas colunas de $X$. Como, para exemplo da Tabela $11, C\left(P^{n}\right) \subset C(X)$ e $C\left(P^{m}\right) \subset C(X)$, o delineamento é ortogonal. Logo $R(\beta \mid \mu)=R(\beta \mid \mu, \alpha, \gamma)=108,8889$ e $R(\gamma \mid \mu)=R(\gamma \mid \mu, \alpha, \beta)=2480,6667$. Para a verificação numérica dessas igualdades, inverteu-se a ordem de entrada dos fatores no modelo, declarandose em primeiro lugar, respectivamente, o fator $B$ (Tabela 14) e o fator $C$ (Tabela 15).

Tabela 13. Análise de variância do delineamento ortogonal desbalanceado, descrito na Tabela 11, segundo o modelo $y_{i j k l}=\mu+\alpha_{i}+\beta_{j}+\gamma_{k}+e_{i j k l}$, declarando $A$ como primeiro fator.

\begin{tabular}{|c|c|c|c|c|}
\hline Fontes & $g l$ & S.Q. Tipo I & Q.M. & $R()$. \\
\hline A & 1 & 12,00000000 & 1).00000000 & $R(\alpha \mid \mu)$ \\
\hline B & 1 & 1088,88888889 & $=088,88888889$ & $R(\beta \mid \mu, \alpha)$ \\
\hline C & 1 & 2480,66666667 & 2480,666666667 & $R(\gamma \mid \mu, \alpha, \beta)$ \\
\hline Fontes & $g l$ & S.Q. Tipo II & Q.M. & $R(\cdot)$ \\
\hline$A$ & 1 & 12,00000000 & 12,00000000 & $R(\alpha \mid \mu, \beta, \gamma)$ \\
\hline B & 1 & 1088,88888889 & 1088,88888889 & $R(\beta \mid \mu, \alpha, \gamma)$ \\
\hline c & 1 & 2480,66666667 & 2480,66666667 & $R(\gamma \mid \mu, \alpha, \beta)$ \\
\hline Fontes & $g l$ & SQ Tipo III $=I V$ & Q.M. & $R()$. \\
\hline A & 1 & 12,00000000 & 12,00000000 & $R(\dot{\alpha} \mid \dot{\mu}, \dot{\beta}, \dot{\gamma})$ \\
\hline B & 1 & 1088,88888889 & 1088,88888889 & $R(\dot{\beta} \mid \dot{\mu}, \dot{\alpha}, \dot{\gamma})$ \\
\hline $\mathrm{C}$ & 1 & 2480,666666667 & 2480,66666667 & $R(\dot{\gamma} \mid \dot{\mu}, \dot{\alpha}, \dot{\beta})$ \\
\hline
\end{tabular}


Tabela 14. Análise de variância do delineamento ortogonal desbalanceado, descrito na Tabela 11, segundo o modelo $y_{i j k l}=\mu+\beta_{j}+\alpha_{i}+\gamma_{k}+e_{i j k l}$, declarando $B$ como primeiro fator.

\begin{tabular}{lcrrl}
\hline Fontes & gl & S.Q. Tipo I & $Q . M$. & $R(\cdot)$ \\
\hline B & 1 & 1088,88888889 & 1088,88888889 & $\boldsymbol{R}(\beta \mid \mu)$ \\
A & 1 & 12,00000000 & 12,0000000 & $R(\alpha \mid \mu, \beta)$ \\
C & 1 & 2480,66666667 & 2480,66666667 & $R(\gamma \mid \mu, \alpha, \beta)$ \\
\hline Fontes & $g l$ & S.Q. Tipo II & $Q . M$. & $R(\cdot)$ \\
\hline B & 1 & 1088,88888889 & 1088,88888889 & $R(\beta \mid \mu, \alpha, \gamma)$ \\
A & 1 & 12,00000000 & 12,0000000 & $R(\alpha \mid \mu, \beta, \gamma)$ \\
C & 1 & 2480,66666667 & 2480,66666667 & $R(\gamma \mid \mu, \alpha, \beta)$ \\
\hline Fontes & $g l$ & S.Q. Tipo III=IV & $Q . M$. & $R(\cdot)$ \\
\hline B & 1 & 1088,88888889 & 1088,88888889 & $R(\dot{\beta} \mid \dot{\mu}, \dot{\alpha}, \dot{\gamma})$ \\
A & 1 & 12,00000000 & 12,00000000 & $R(\dot{\alpha} \mid \dot{\mu}, \dot{\beta}, \dot{\gamma})$ \\
C & 1 & 2480,66666667 & 2480,66666667 & $R(\dot{\gamma} \mid \dot{\mu}, \dot{\alpha}, \dot{\beta})$ \\
\hline
\end{tabular}

Uma das grandes implicações da ortogonalidade é que nos delineamentos ortogonais, por construção, hả apenas uma partição ortogonal da soma de quadrados de parâmetros. Isso implica que a ordem de entrada dos fatores no modelo, como se verifica nas Tabelas 13, 14 e 15, passa a não se fazer necessária, quando as somas de quadrados associadas às hipóteses dos tipos I e II são requeridas pelo pesquisador. Por conseqüência indireta da ortogonalidade, as somas de quadrados dos tipos III são iguais às do tipo IV e pela ausência de interações tipo II é igual ao tipo III. Uma avaliação menos cuidadosa das Tabelas 13, 14 e 15 poderia levar a uma interpretação dos resultados como feita para conjuntos balanceados. Essa identidade entre os conjuntos balanceados e desbalanceados para modelos sem interações revela a grande aplicação e facilidade de interpretação quando os experimentos são planejados de tal forma a gerar freqüências proporcionais. 
Tabela 15. Análise de variância do delineamento ortogonal desbalanceado, descrito na Tabela 11, segundo o modelo $y_{i j k l}=\mu+\gamma_{k}+\alpha_{i}+\beta_{j}+e_{i j k l}$, declarando $C$ como primeiro fator.

\begin{tabular}{|c|c|c|c|c|}
\hline Fontes & $g l$ & S.Q. Tipo I & Q.M. & $R()$. \\
\hline C & 1 & 2480,66666667 & 2480,66665667 & $R(\gamma \mid \mu)$ \\
\hline A & 1 & 12,00000000 & 12,00000000 & $R(\alpha \mid \mu, \gamma)$ \\
\hline $\mathrm{B}$ & 1 & 1088,88888889 & 1088,88888889 & $R(\beta \mid \mu, \alpha, \gamma)$ \\
\hline Fontes & $g l$ & S.Q. Tipo II & Q.M. & $R()$. \\
\hline $\mathrm{c}$ & 1 & 2480,66666667 & 2480,66666667 & $\boldsymbol{R}(\gamma \mid \mu, \alpha, \beta)$ \\
\hline A & $i$ & 12,00000000 & 12,00000000 & $R(\alpha \mid \mu, \beta, \gamma)$ \\
\hline B & 1 & 1088,88888889 & 1088,88888889 & $R(\beta \mid \mu, \alpha, \gamma)$ \\
\hline Fontes & $g l$ & S.Q. Tipo II & Q.M. & $R(\cdot)$ \\
\hline c & 1 & 2480,66666667 & 2400,66666667 & $\boldsymbol{R}(\dot{\gamma} \mid \dot{\mu}, \dot{\alpha}, \dot{\beta})$ \\
\hline A & 1 & 12,00000000 & 12,00000000 & $R(\dot{\alpha} \mid \dot{\mu}, \dot{\beta}, \dot{\gamma})$ \\
\hline B & 1 & 1088,88888889 & :088, 88888889 & $R(\dot{\beta} \mid \dot{\mu}, \dot{\alpha}, \dot{\gamma})$ \\
\hline
\end{tabular}

Neste estudo, a ordem de entrada dos fatores no modelo foi importante, pois mostrou as conseqüências da ortogonalidade para todos os fatores do modelo. Entretanto, após o planejamento de delineamentos ortogonais, essa ordem de entrada dos fatores no modelo passa a não ser mais importante o que amplia a utilização dos sistemas computacionais.

\section{b.2 Modelo com interações}

Para a discussão dos modelos com três fatores e interações, foram utilizados os mesmos dados da Tabela 11 e formuladas hipóteses dos tipos I e II apenas para o fator $A$, segundo o critério de Searle (1987). O modelo adotado para a construção das hipóteses foi o de médias de caselas caracterizado por $y_{i j k l}=\mu_{i j k}+e_{i j k l}($ Speed, Hocking e Hackney, 1978), como mostra a Tabela 16. 
Tabela 16. Representação do modelo de médias de caselas do delineamento ortogonal desbalanceado descrito na Tabela 11.

\begin{tabular}{|c|c|c|c|c|}
\hline \multirow[b]{4}{*}{ Fator $A$} & \multicolumn{4}{|c|}{ Fator $B$} \\
\hline & \multicolumn{2}{|c|}{$j=1$} & \multicolumn{2}{|c|}{$\dot{j} 2$} \\
\hline & \multicolumn{2}{|c|}{ Fator $C$} & \multicolumn{2}{|c|}{ Fator $C$} \\
\hline & $k=1$ & $k=2$ & $k=1$ & $k=2$ \\
\hline$i=1$ & $\mu_{111}{ }^{(1)}$ & $\mu_{112}^{(1)}$ & $\mu_{121}{ }^{(3)}$ & $\mu_{122}{ }^{(3)}$ \\
\hline$i=2$ & $\mu_{211}{ }^{(2)}$ & $\mu_{212}^{(2)}$ & $\mu_{221}{ }^{(6)}$ & $\mu_{222}{ }^{(6)}$ \\
\hline
\end{tabular}

\section{a Hipótese do tipo I para o fator $A$}

A formulação da hipótese sobre médias ponderadas de delineamentos ortogonais mostra que quando a ponderação para um determinado fator é realizada, automaticamente já ocorre o ajuste para os outros fatores, fazendo com que uma hipótese sobre médias ponderadas iguale-se à hipótese sobre médias ponderadas ajustadas.

$H_{o}^{1-A}: \frac{\mu_{111}+\mu_{112}+3 \mu_{121}+3 \mu_{122}}{8}=\frac{2 \mu_{211}+2 \mu_{212}+6 \mu_{221}+6 \mu_{222}}{16}$

\section{a. 1 Modelo-M}

$H_{o}^{1-A}: \mu_{111}+\mu_{112}+3 \mu_{121}+3 \mu_{122}-\mu_{211}-\mu_{212}-3 \mu_{221}-3 \mu_{222}=0$

\section{a.2 Modelo-S}

$$
\begin{aligned}
H_{O}^{1-A}: & \alpha_{1}+1 / 8\left(2 \varphi_{11}+6 \varphi_{12}+4 \eta_{11}+4 \eta_{12}+\rho_{111}+\rho_{112}+3 \rho_{121}+3 \rho_{122}\right)= \\
= & \alpha_{2}+1 / 8\left(2 \varphi_{21}+6 \varphi_{22}+4 \eta_{21}+4 \eta_{22}+\rho_{211}+\rho_{212}+3 \rho_{221}+3 \rho_{222}\right)
\end{aligned}
$$

Apesar da ortogonalidade, a função estimável do tipo I para o fator $A$ com a presença de parâmetros de interações, dificulta a interpretação dos resultados, tornando-se confusa e complexa.

Conforme descrito em P.6, a ortogonalidade fez com que as funções estimáveis para os efeitos principais apresentassem parâmetros do 
próprio fator e parâmetros das interações onde o fator está presente, caracteristica anteriormente discutida para os conjuntos balanceados e presente nos conjuntos desbalanceados. Portanto, para o fator $A$ apresentado no item a.2, tem-se funçōes dos seguintes parâmetros: $\alpha, \varphi, \eta$ e $\rho$ que são parâmetros correspondentes ao próprio fator, à interação $A * B$, à interação $A^{*} C$ e à interação $A^{*} B^{*} C$, respectivamente (Quadro 14).

\section{b Hipótese tipo II para o fator $A$}

$$
H_{o}^{2-A}: \frac{\mu_{111}+\mu_{112}+3 \mu_{121}+3 \mu_{122}}{8}=\frac{\bar{C}_{1}+\bar{C}_{2}+3 \bar{C}_{3}+3 \bar{C}_{4}}{8}
$$

onde:

$$
\bar{C}_{1}=\frac{\mu_{111}+2 \mu_{211}}{3} ; \bar{C}_{2}=\frac{\mu_{112}+2 \mu_{212}}{3} ; \bar{C}_{3}=\frac{3 \mu_{121}+6 \mu_{221}}{9} \text { e } \bar{C}_{4}=\frac{3 \mu_{122}+6 \mu_{222}}{9}
$$

Trabalhando com o segundo termo da hipótese chega-se a seguinte igualdade,

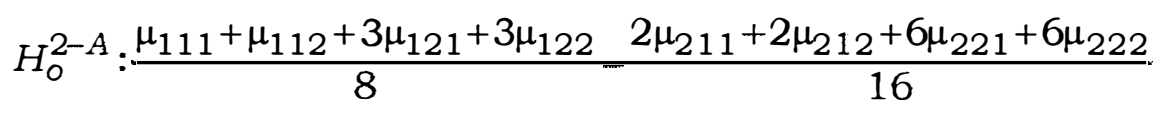

\section{b. 1 Modelo-M}

$$
H_{o}^{2-A}: \mu_{111}+\mu_{112}+3 \mu_{121}+3 \mu_{122}-\mu_{211}-\mu_{212}-3 \mu_{221}-3 \mu_{222}=0
$$

\section{b. 2 Modelo-S}

$$
\begin{aligned}
H_{o}^{2-A}: & \alpha_{1}+1 / 8\left(2 \varphi_{11}+6 \varphi_{12}+4 \eta_{11}+4 \eta_{12}+\rho_{111}+\rho_{112}+3 \rho_{121}+3 \rho_{122}\right)= \\
= & \alpha_{2}+1 / 8\left(2 \varphi_{21}+6 \varphi_{22}+4 \eta_{21}+4 \eta_{22}+\rho_{211}+\rho_{212}+3 \rho_{221}+3 \rho_{222}\right)
\end{aligned}
$$


Quadro 14. Funções estimáveis fornecidas pelo Proc GLM do SAS e respectivas hipóteses sobre o fator $A$, para os dados da Tabela 11 , considerando o modelo com interaçōes.

\begin{tabular}{|c|c|c|c|c|c|}
\hline$T$ 니 & $I-A=$ & Tipo II-A & Tif & $\boldsymbol{D U}-\boldsymbol{A}$ & Tipo $\Gamma V-A$ \\
\hline $\begin{array}{l}\text { Efeitc } \\
\text { INTER }\end{array}$ & & $\begin{array}{l}\text { Coeficientes } \\
0\end{array}$ & $\begin{array}{l}\text { Efeitc } \\
\text { INTERC }\end{array}$ & & $\begin{array}{l}\text { Coeficientes } \\
0\end{array}$ \\
\hline$A$ & $\begin{array}{l}1 \\
2\end{array}$ & $\begin{array}{l}\mathrm{L} 2 \\
-\mathrm{L} 2\end{array}$ & $\dot{A}$ & $\begin{array}{l}1 \\
2\end{array}$ & $\begin{array}{l}\mathrm{L} 2 \\
-\mathrm{I} 2\end{array}$ \\
\hline B & $\begin{array}{l}1 \\
2\end{array}$ & $\begin{array}{l}0 \\
0\end{array}$ & $\mathrm{~B}$ & $\begin{array}{l}1 \\
2\end{array}$ & $\begin{array}{l}0 \\
0\end{array}$ \\
\hline $\mathrm{C}$ & $\begin{array}{l}1 \\
2\end{array}$ & $\begin{array}{l}0 \\
0\end{array}$ & c & $\begin{array}{l}1 \\
2\end{array}$ & $\begin{array}{l}0 \\
0\end{array}$ \\
\hline$A \star B$ & $\begin{array}{ll}1 & 1 \\
1 & 2 \\
2 & 1 \\
2 & 2\end{array}$ & $\begin{array}{l}0,25 * \mathrm{~L} 2 \\
0,75 * \mathrm{~L} 2 \\
-0,25 * \mathrm{~L} 2 \\
-0,75 * \mathrm{I} 2\end{array}$ & $A * B$ & $\begin{array}{ll}1 & 1 \\
1 & 2 \\
2 & 1 \\
2 & 2\end{array}$ & $\begin{array}{l}0,5 * L 2 \\
0,5 * L 2 \\
-0,5 * L 2 \\
-0,5 * L 2\end{array}$ \\
\hline$A * C$ & $\begin{array}{ll}1 & 1 \\
1 & 2 \\
2 & 1 \\
2 & 2\end{array}$ & $\begin{array}{l}0,5 * \mathrm{~L} 2 \\
0,5 * \mathrm{~L} 2 \\
-0,5 * \mathrm{~L} 2 \\
-0,5 * \mathrm{~L} 2\end{array}$ & $A * C$ & $\begin{array}{ll}1 & 1 \\
1 & 2 \\
2 & 1 \\
2 & 2\end{array}$ & $\begin{array}{l}0,5 * \mathrm{~L} 2 \\
0,5 * \mathrm{~L} 2 \\
-0,5 * \mathrm{~L} 2 \\
-0,5 * \mathrm{~L} 2\end{array}$ \\
\hline$B * C$ & $\begin{array}{ll}1 & 1 \\
1 & 2 \\
2 & 1 \\
2 & 2\end{array}$ & $\begin{array}{l}0 \\
0 \\
0 \\
0\end{array}$ & $\Xi * C$ & $\begin{array}{ll}1 & 1 \\
1 & 2 \\
2 & 1 \\
2 & 2\end{array}$ & $\begin{array}{l}0 \\
0 \\
0 \\
0\end{array}$ \\
\hline$A * B * C$ & $\begin{array}{lll}1 & 1 & 1 \\
1 & 1 & 2 \\
1 & 2 & 1 \\
1 & 2 & 2 \\
2 & 1 & 1 \\
2 & 1 & 2 \\
2 & 2 & 1 \\
2 & 2 & 2\end{array}$ & $\begin{array}{l}0,125 * \mathrm{~L} 2 \\
0,125 * \mathrm{~L} 2 \\
0,375 * \mathrm{~L} 2 \\
0,375 * \mathrm{~L} 2 \\
-0,125 * \mathrm{~L} 2 \\
-0,125 * \mathrm{~L} 2 \\
-0,375 * \mathrm{~L} 2 \\
-0,375 * \mathrm{~L} 2\end{array}$ & $\therefore * B \star C$ & $\begin{array}{lll}1 & 1 & 1 \\
1 & 1 & 2 \\
1 & 2 & 1 \\
1 & 2 & 2 \\
2 & 1 & 1 \\
2 & 1 & 2 \\
2 & 2 & 1 \\
2 & 2 & 2\end{array}$ & $\begin{array}{l}0,25 * \mathrm{~L} 2 \\
0,25 * \mathrm{~L} 2 \\
0,25 * \mathrm{~L} 2 \\
0,25 * \mathrm{~L} 2 \\
-0,25 * \mathrm{~L} 2 \\
-0,25 * \mathrm{~L} 2 \\
-0,25 * \mathrm{~L} 2 \\
-0,25 * \mathrm{~L} 2\end{array}$ \\
\hline $\begin{aligned} H_{o}^{1-A}=H_{o}^{2-A} & : \alpha_{1} \\
& +\rho_{11} \\
& +4 \eta_{2}\end{aligned}$ & $\begin{array}{l}\left(2 \varphi_{11}+\right. \\
\rho_{121}+3 \\
\eta_{22}+\rho_{2}\end{array}$ & $\begin{array}{l}\rho_{12}+4 \eta_{11}+4 \eta_{12}+\rho_{111}+ \\
22)=\alpha_{2}+1 / 8\left(2 \varphi_{21}+6 \varphi_{22}+\right. \\
\left.1+\rho_{212}+3 \rho_{221}+3 \rho_{222}\right)\end{array}$ & $\begin{array}{r}H_{o}^{3-A}=H_{o}^{4-A}: c \\
+p \\
+2\end{array}$ & $\begin{array}{l}1 / 4\left(2 \varphi_{1}\right. \\
+\rho_{121}+ \\
+2 \eta_{22}\end{array}$ & $\begin{array}{l}2 \varphi_{12}+2 \eta_{11}+2 \eta_{12}+\rho_{111}+ \\
22)=\alpha_{2}+1 / 4\left(2 \varphi_{21}+2 \varphi_{22}+\right. \\
\left.211+\rho_{212}+\rho_{221}+\rho_{222}\right)\end{array}$ \\
\hline
\end{tabular}


Quadro 15. Funções estimáveis fornecidas pelo Proc GLM do SAS e respectivas hipóteses sobre a interação $A^{*} B$, para os dados da Tabela 11, considerando o modelo com interações.

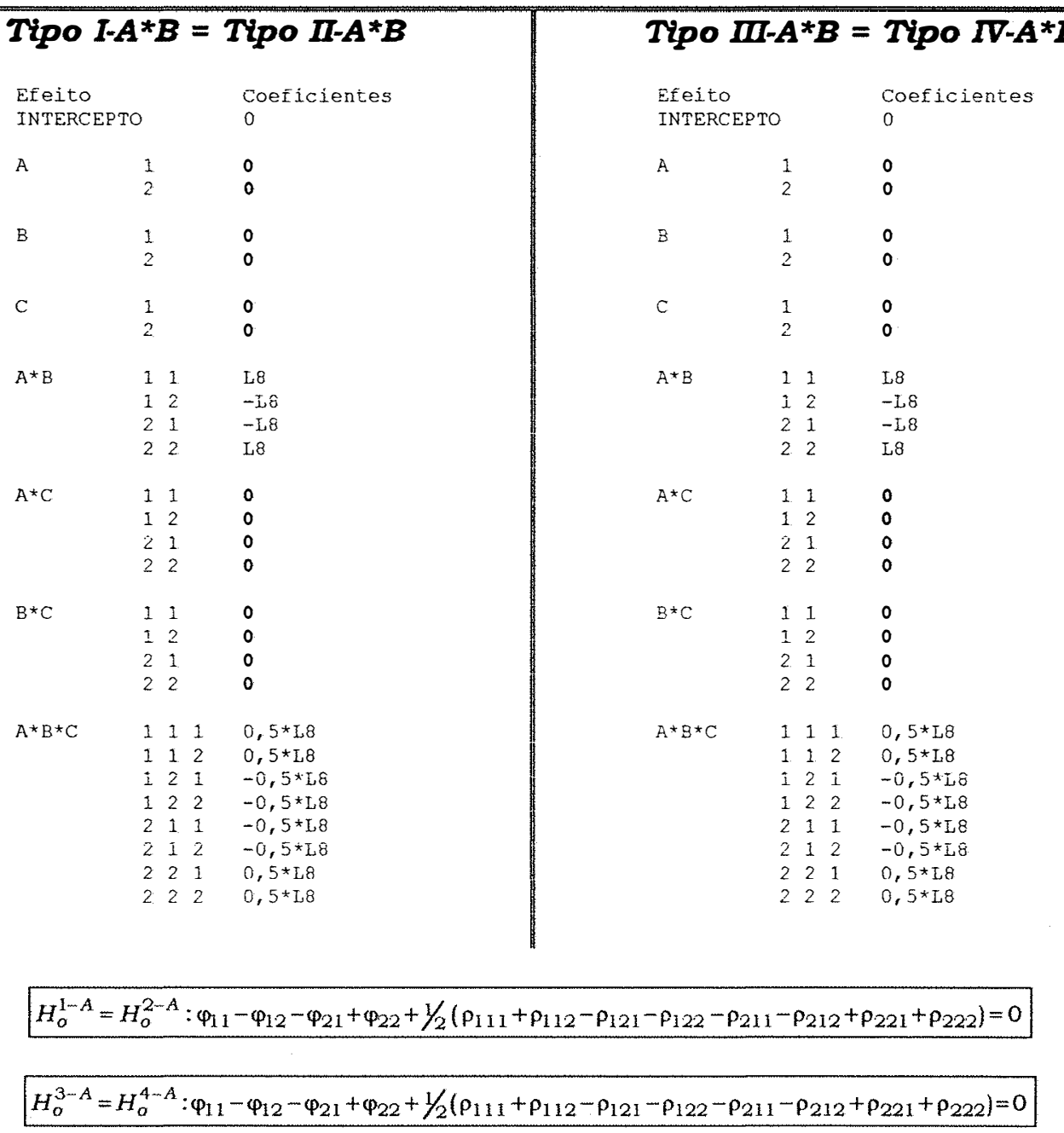

O Quadro 14 revela para o fator $A$ a esperada igualdade entre as funções estimáveis dos tipos I e II pela ortogonalidade e a igualdade entre os ipos III e IV devida a ausência de caselas vazias. Entretanto, um resultado pouco comum ocorreu para a interaçāo $A{ }^{*} B$ que apresentou igualdade entre as funções estimáveis dos tipos II e III mesmo o conjunto sendo desbalanceado. Esse resultado ocorre porque na hipótese do ipo II, apenas para a interação $A * B$, as freqüências são constantes, ou seja, hipóteses sob médias ponderadas ajustadas e não ponderadas ajustadas coincidem, como se observa no Quadro 
15. Esse resultado não ocorre para as outras interações pois na hipótese do tipo II as freqüências não são constantes.

As Tabelas 17, 18 e 19 mostram a igualdade entre as somas de quadrados associadas às hipóteses dos tipos I e II para todos os efeitos principais e interações do modelo, apresentando uma variação na ordem de entrada dos fatores e das interações. Além da igualdade entre as somas de quadrados associadas às hipóteses dos tipos I e II, são iguais as seguintes decomposições para o fator $A: \quad R(\alpha \mid \mu)=R(\alpha \mid \mu, \beta)=R(\alpha \mid \mu, \gamma)=R(\alpha \mid \mu, \beta, \gamma)=$ $=12,0000$. Ademais, como o modelo contêm interações, a ordem de entrada das interações no modelo passa a ser um recurso didático importante para mostrar, que além da igualdade entre as somas de quadrados associadas as hipóteses dos tipos I e II para as interações são iguais as seguintes decomposições para $\quad A^{*} B: \quad R(\varphi \mid \mu, \alpha, \beta, \gamma)=R(\varphi \mid \mu, \alpha, \beta, \gamma, \eta)=R(\varphi \mid \mu, \alpha, \beta, \gamma, \tau)=$ $=R(\varphi \mid \mu, \alpha, \beta, \gamma, \eta, \tau)=75,1111$.

Nos modelos com dois fatores, como só há uma interação, não faz sentido a discussão entre hipóteses do tipo I e II para a interação, que passa a ser importante em modelos com mais de dois fatores. A Tabela 17 apresenta as somas de quadrados associadas às hipóteses do tipo III para os efeitos principais e interações, mostrando a diferença com o tipo II devida à presença de interações no modelo $\mathrm{e}$ a igualdade entre as somas de quadrados associadas às hipóteses dos tipos III e IV devida à ausência de caselas vazias.

Os resultados da Tabela 18 mostram, para o fator $B$, que $R(\beta \mid \mu)=$ $=R(\beta \mid \mu, \alpha, \gamma)=1088,8889$ e para a interação $A^{*} C$, que $R(\eta \mid \mu, \alpha, \beta, \gamma)=$ $=R(\eta \mid \mu, \alpha, \beta, \gamma, \varphi, \tau)=90,3333$, enquanto que na Tabela 19 observa-se que para $o$ fator $C, \quad R(\gamma \mid \mu)=R(\gamma \mid \mu, \alpha, \beta)=2480,6667$ e para a interação $B^{*} C$ $R(\tau \mid \mu, \alpha, \beta, \gamma)=R(\tau \mid \mu, \alpha, \beta, \gamma, \eta, \varphi)=304,2222$. 
Tabela 17. Análise de variância do delineamento ortogonal desbalanceado, descrito na Tabela 11, segundo o modelo $y_{i j k l}=\mu+\alpha_{i}+\beta_{j}+\gamma_{k}+\varphi_{i j}+\eta_{i k}+\tau_{j k}+\rho_{i j k}+e_{i j k l}$, com $A$ sendo o primeiro fator declarado entre os efeitos principais e $A * B$ a primeira entre as interaçōes.

\begin{tabular}{|c|c|c|c|c|}
\hline Fontes & $g l$ & S.Q. Tipo I & Q.M. & $R()$. \\
\hline A & 1 & 12,00000000 & 12,00000000 & $R(\alpha \mid \mu)$ \\
\hline B & 1 & 1088,888888889 & 1088,888888889 & $R\left(\left.\beta\right|_{\mu, \alpha)}\right.$ \\
\hline c & 1 & 2480,66666667 & 2480,666666667 & $R(\gamma \mid \mu, \alpha, \beta)$ \\
\hline$A \star B$ & 1 & 75,11111111 & 75,11111111 & $R(\varphi \mid \mu, \alpha, \beta, \gamma)$ \\
\hline$A \star C$ & 1 & 901,33333333 & 901,33333333 & $R(\eta \mid \mu, \alpha, \beta, \gamma, \varphi)$ \\
\hline$B \star C$ & 1 & 304,22222222 & 304,22222222 & $R(\tau \mid \mu, \alpha, \beta, \gamma, \varphi, \eta)$ \\
\hline$A * B * C$ & 1 & 641,77777778 & 641,77777778 & $R(\rho \mid \mu, \alpha, \beta, \gamma, \varphi, \eta, \tau)$ \\
\hline Fontes & $g l$ & S.Q. Tipo II & Q.M. & $R()$. \\
\hline A & 1 & 12,00000000 & 12,00000000 & $R(\alpha \mid \mu, \beta, \gamma)$ \\
\hline B & 1 & 1088,88888889 & 1088,888888889 & $R(\beta \mid \mu, \alpha, \gamma)$ \\
\hline c & 1 & 2480,66666667 & 2480,66666667 & $R(\gamma \mid \mu, \alpha, \beta)$ \\
\hline$A \star B$ & 1 & 75,11111111 & 75,11111111 & $R(\Phi \mid \mu, \alpha, \beta, \gamma, \eta, \tau)$ \\
\hline$A^{\star} \mathrm{C}$ & 1 & 901,33333333 & 901,33333333 & $R(\eta \mid \mu, \alpha, \beta, \gamma, \varphi, \tau)$ \\
\hline$B \star C$ & 1 & 304,22222222 & 304,22222222 & $R(\tau \mid \mu, \alpha, \beta, \gamma, \varphi, \tau)$ \\
\hline$A * B * C$ & 1 & 641,77777778 & 641,77777778 & $R(\rho \mid \mu, \alpha, \beta, \gamma, \varphi, \eta, \tau)$ \\
\hline Fontes & $g l$ & SQ Tipo III $=I V$ & Q.M. & $R()$. \\
\hline A & 1 & 53,77777778 & 53,77777778 & $R(\dot{\alpha} \mid \dot{\mu}, \dot{\beta}, \dot{\gamma})$ \\
\hline B & 1 & 1156,00000000 & $\because: 56,00000000$ & $R(\dot{\beta} \mid \dot{\mu}, \dot{\alpha}, \dot{\gamma})$ \\
\hline c & 1 & 784,00000000 & 784,00000000 & $R(\dot{\gamma} \mid \dot{\mu}, \dot{\alpha}, \dot{\beta})$ \\
\hline$A \star B$ & 1 & 75,11111111 & 75,11111111 & $R(\dot{\varphi} \mid \dot{\mu}, \dot{\alpha}, \dot{\beta}, \dot{\gamma}, \dot{\eta}, \dot{\tau})$ \\
\hline$A^{\star} \mathrm{C}$ & 1 & 177,77777778 & 177,77777778 & $R(\dot{\eta} \mid \dot{\mu}, \dot{\alpha}, \dot{\beta}, \dot{\gamma}, \dot{\varphi}, \dot{\tau})$ \\
\hline$B^{\star} \mathrm{C}$ & 1 & 64,00000000 & 64,00000000 & $R(\dot{\tau} \mid \dot{\mu}, \dot{\alpha}, \dot{\beta}, \dot{\gamma}, \dot{\varphi}, \dot{\tau})$ \\
\hline$A \star B \star C C$ & 1 & 641,77777778 & 641,77777778 & $R(\dot{\rho} \mid \dot{\mu}, \dot{\alpha}, \dot{\beta}, \dot{\gamma}, \dot{\varphi}, \dot{\eta}, \dot{\tau})$ \\
\hline
\end{tabular}


Tabela 18. Análise de variância do delineamento ortogonal desbalanceado, descrito na Tabela 11, segundo o modelo $y_{i j k l}=\mu+\beta_{j}+\alpha_{i}+\gamma_{k}+\eta_{i k}+\varphi_{i j}+\tau_{j k}+\rho_{i j k}+e_{i j k l}$, sendo $B$ o primeiro fator declarado entre os efeitos principais e $A^{*} C$ a primeira entre as interações.

\begin{tabular}{|c|c|c|c|c|}
\hline Fontes & $g l$ & S.Q. Tipo I & Q.M. & $R()$. \\
\hline B & 1 & 1088,88888889 & 1008,00000003 & $\boldsymbol{R}(\beta \mid \mu)$ \\
\hline A & 1 & 12,00000000 & 12,00000000 & $R(\alpha \mid \mu, \beta)$ \\
\hline C & 1 & 2480,66666667 & 2480,66666667 & $R(\gamma \mid \mu, \alpha, \beta)$ \\
\hline$A \star C$ & 1 & 901,33333333 & 901,33333333 & $R(\eta \mid \mu, \alpha, \beta, \gamma)$ \\
\hline$A^{\star B} B$ & 1 & 75,11111111 & 75,11111111 & $R(\varphi \mid \mu, \alpha, \beta, \gamma, \eta)$ \\
\hline $\mathrm{B}^{\star} \mathrm{C}$ & 1 & 304,22222222 & 304,22222222 & $R(\tau \mid \mu, \alpha, \beta, \gamma, \eta, \varphi)$ \\
\hline$A \star B \star C$ & 1 & 641,77777778 & 641,77777778 & $R(\rho \mid \mu, \alpha, \beta, \gamma, \varphi, \eta, \tau)$ \\
\hline Fontes & $g l$ & S.Q. Tipo II & Q.M. & $R()$. \\
\hline B & 1 & 1088,88888889 & 1089,88888889 & $R(\beta \mid \mu, \alpha, \gamma)$ \\
\hline A & 1 & 12,00000000 & 12,00000000 & $R(\alpha \mid \mu, \beta, \gamma)$ \\
\hline c & 1 & 2480,666666667 & 2480,666666667 & $R(\gamma \mid \mu, \alpha, \beta)$ \\
\hline$A * C$ & 1 & 901,33333333 & 901,33333333 & $\boldsymbol{R}(\eta \mid \mu, \alpha, \beta, \gamma, \varphi, \tau)$ \\
\hline$A^{\star} B$ & 1 & 75,11111111 & 75,11111111 & $R(\varphi \mid \mu, \alpha, \beta, \gamma, \eta, \tau)$ \\
\hline$B{ }^{*} C$ & 1 & 304,22222222 & 304,22222222 & $R(\tau \mid \mu, \alpha, \beta, \gamma, \eta, \varphi)$ \\
\hline$A * B * C$ & 1 & 641,77777778 & 641,77777778 & $R(\rho \mid \mu, \alpha, \beta, \gamma, \varphi, \eta, \tau)$ \\
\hline Fontes & $g l$ & SQ Tipo III =IV & Q.M. & $R()$. \\
\hline $\mathbf{B}$ & 1 & 1156,00000000 & 1156,00000000 & $R(\dot{\beta} \mid \dot{\mu}, \dot{\alpha}, \dot{\gamma})$ \\
\hline A & 1 & 53,77777778 & 53,77777778 & $R(\dot{\alpha} \mid \dot{\mu}, \dot{\beta}, \dot{\gamma})$ \\
\hline $\mathrm{C}$ & 1 & 784,00000000 & 784,00000000 & $R(\dot{\gamma} \mid \dot{\mu}, \dot{\alpha}, \dot{\beta})$ \\
\hline$A * C$ & 1 & 177,77777778 & 177,77777778 & $R(\dot{\eta} \mid \dot{\mu}, \dot{\alpha}, \dot{\beta}, \dot{\gamma}, \dot{\varphi}, \dot{\tau})$ \\
\hline$A * B$ & 1 & 75,11111111 & 75,11111111 & $R(\dot{\varphi} \mid \dot{\mu}, \dot{\alpha}, \dot{\beta}, \dot{\gamma}, \dot{\eta}, \dot{\tau})$ \\
\hline$B{ }^{*} \mathrm{C}$ & 1 & 64,00000000 & 64,00000000 & $R(\dot{\tau} \mid \dot{\mu}, \dot{\alpha}, \dot{\beta}, \dot{\gamma}, \dot{\varphi}, \dot{\tau})$ \\
\hline$A A^{*}{ }^{*} C$ & 1 & 641,77777778 & 641,77777778 & $R(\dot{\rho} \mid \dot{\mu}, \dot{\alpha}, \dot{\beta}, \dot{\gamma}, \dot{\varphi}, \dot{\eta}, \dot{\tau})$ \\
\hline
\end{tabular}


Tabela 19. Análise de variância do delineamento ortogonal desbalanceado, descrito na Tabela 11, segundo o modelo $y_{i j k l}=\mu++\gamma_{k}++\beta_{j}+\alpha_{i}++\tau_{j k}+\eta_{i k}+\varphi_{i j}+\rho_{i j k}+e_{i j k l}$, sendo $C$ o primeiro fator declarado entre os efeitos principais e $B^{\star} C$ a primeira entre as interações.

\begin{tabular}{|c|c|c|c|c|}
\hline Fontes & $g l$ & S.Q. Tipo I & Q.M. & $R()$. \\
\hline c & 1 & 2480,66666667 & 2400,66666667 & $\boldsymbol{R}(\gamma \mid \mu)$ \\
\hline B & 1 & 1088,88888889 & 1088,88888889 & $R(\beta \mid \mu, \gamma)$ \\
\hline A & 1 & 12,00000000 & 12,00000000 & $R(\alpha \mid \mu, \beta, \gamma)$ \\
\hline$B * C$ & 1 & 304,22222222 & 304,22222222 & $\boldsymbol{R}(\tau \mid \mu, \alpha, \beta, \gamma)$ \\
\hline$A \star C$ & 1 & 901,33333333 & 901,33333333 & $R(\eta \mid \mu, \alpha, \beta, \gamma, \tau)$ \\
\hline$A{ }^{\star} B$ & 1 & 75,11111111 & 75,11111111 & $R(\varphi \mid \mu, \alpha, \beta, \gamma, \eta, \tau)$ \\
\hline$A{ }^{*} B{ }^{*} C$ & 1 & 641,77777778 & 641,77777778 & $R(\rho \mid \mu, \alpha, \beta, \gamma, \varphi, \eta, \tau)$ \\
\hline Fontes & $g l$ & S.Q. Tipo II & Q.M. & $R()$. \\
\hline c & 1 & 2480,66666667 & $2480,6 \varepsilon 5555557$ & $\boldsymbol{R}(\gamma \mid \mu, \alpha, \beta)$ \\
\hline B & 1 & 1088,88888889 & 1088,88888889 & $R\left(\left.\beta\right|_{\mu, \alpha, \gamma)}\right.$ \\
\hline A & 1 & 12,00000000 & 12,00000000 & $R(\alpha \mid \mu, \beta, \gamma)$ \\
\hline$B * C$ & 1 & 304,22222222 & 304,22222222 & $R(\tau \mid \mu, \alpha, \beta, \gamma, \eta, \varphi)$ \\
\hline$A * C$ & 1 & 901,33333333 & 901,33333333 & $R(\eta \mid \mu, \alpha, \beta, \gamma, \varphi, \tau)$ \\
\hline$A A^{\star}$ & 1 & 75,11111111 & 75,11111111 & $R(\varphi \mid \mu, \alpha, \beta, \gamma, \eta, \tau)$ \\
\hline$A{ }^{\star} B{ }^{\star} C$ & 1 & 641,77777778 & 641,77777778 & $R(\rho \mid \mu, \alpha, \beta, \gamma, \varphi, \eta, \tau)$ \\
\hline Fontes & $g l$ & SQ Tipo $I I=I V$ & Q.M. & $R()$. \\
\hline $\bar{c}$ & 1 & 784,00000000 & 784,00000000 & $R(\dot{\gamma} \mid \dot{\mu}, \dot{\alpha}, \dot{\beta})$ \\
\hline B & 1 & 1156,00000000 & 1156,00000000 & $R(\dot{\beta} \mid \dot{\mu}, \dot{\alpha}, \dot{\gamma})$ \\
\hline A & 1 & 53,77777778 & 53,77777778 & $R(\dot{\alpha} \mid \dot{\mu}, \dot{\beta}, \dot{\gamma})$ \\
\hline$B \star C$ & 1 & 64,000000000 & 64,00000000 & $R(\dot{\tau} \mid \dot{\mu}, \dot{\alpha}, \dot{\beta}, \dot{\gamma}, \dot{\varphi}, \dot{\tau})$ \\
\hline$A{ }^{*} \mathrm{C}$ & 1 & 177,77777778 & 177,77777778 & $R(\dot{\eta} \mid \dot{\mu}, \dot{\alpha}, \dot{\beta}, \dot{\gamma}, \dot{\varphi}, \dot{\tau})$ \\
\hline$A \star B$ & 1 & 75,11111111 & 75,11111111 & $R(\dot{\varphi} \mid \dot{\mu}, \dot{\alpha}, \dot{\beta}, \dot{\gamma}, \dot{\eta}, \dot{\tau})$ \\
\hline$A{ }^{*} B{ }^{*} C$ & 1 & 641,77777778 & 641,77777778 & $R(\dot{\rho} \mid \dot{\mu}, \dot{\alpha}, \dot{\beta}, \dot{\gamma}, \dot{\varphi}, \dot{\eta}, \dot{\tau})$ \\
\hline
\end{tabular}

Todas as igualdades entre as somas de quadrados associadas às hipóteses podem ser verificadas de outras maneiras, como por exemplo, aplicando-se a prova de Christensen (1987) baseada na igualdade entre os subespaços gerados pelos projetores e o subespaço gerado pelas colunas do projetor da matriz de delineamento contendo as interações. Uma demonstração dessa igualdade foi apresentada nos modelos sem interação, para o mesmo exemplo, e por isso não está aqui detalhada. 


\subsection{Delineamentos parcialmente ortogonais}

A ortogonalidade parcial confere à forma das funçōes estimáveis e às hipöteses, caracteristicas semelhantes aos delineamentos ortogonais para 0 fator ou fatores cujas freqüências de caselas são proporcionais aos demais fatores. As maiores diferenças entre os dois delineamentos concentra-se nos modelos com interações que, nos delineamentos parcialmente ortogonais, apresentam caracteristicas muito próprias. Nos itens apresentados a seguir são mostrados dois exemplos que incluem modelos com três e quatro fatores com e sem interações, contemplando pelo menos um exemplo das diversas situaçōes apresentadas na metodologia.

\subsubsection{Modelos com três fatores}

Nos modelos com três fatores a ortogonalidade parcial pode gerar três casos distintos, nos quais cada fator, $A, B$ ou $C$ apresenta freqüências de caselas proporcionais aos demais. O 5o exemplo na Tabela 20 apresenta o caso 1 do Quadro 4, na qual a proporcionalidade das freqüências com os demais fatores ocorre com o fator $A$.

\subsubsection{5o Exemplo}

Tabela 20. Dados de porcentagem de germinação de canafistula (Peltophorum dubium Spreng) do delineamento parcialmente ortogonal com três fatores.

\begin{tabular}{|c|c|c|c|c|}
\hline \multirow[b]{4}{*}{ Temperatura (fator $A$ ) } & \multicolumn{4}{|c|}{ Substrato (fator $B$ ) } \\
\hline & \multicolumn{2}{|c|}{$\dot{j} 1$} & \multicolumn{2}{|c|}{$j=2$} \\
\hline & \multicolumn{2}{|c|}{ Dormência ( fator $C$ ) } & \multicolumn{2}{|c|}{ Dormência (fator $C$ ) } \\
\hline & $k=1$ & $k=2$ & $k=1$ & $k=2$ \\
\hline \multirow[t]{3}{*}{$i=1$} & 12 & 28 & 38 & 40 \\
\hline & & & 46 & 46 \\
\hline & & & 42 & \\
\hline \multirow[t]{6}{*}{$i=2$} & 30 & 34 & 12 & 58 \\
\hline & 22 & 26 & 22 & 56 \\
\hline & & & 30 & 62 \\
\hline & & & 12 & 52 \\
\hline & & & 18 & \\
\hline & & & 38 & \\
\hline
\end{tabular}


Para os dados da Tabela 20, através das expressões 15, 20 e 25 foram obtidos os seguintes resultados:

\section{i) Ignorando o fator C}

\begin{tabular}{|c|c|c|c|c|}
\hline \multirow[b]{2}{*}{ Fator B } & \multicolumn{2}{|c|}{ Fator $A$} & \multirow[b]{2}{*}{$n_{. j .}$} & \multirow{2}{*}{$\frac{n_{i j .}}{n_{i^{\prime} .}}=\frac{n_{i^{\prime} j .}}{n_{i^{\prime} j^{\prime} .}}=\frac{2}{5}$ ou $\frac{1}{2}$} \\
\hline & $i=1$ & $i=2$ & & \\
\hline$\dot{j}=1$ & $n_{11 .}=2$ & $n_{21}=4$ & $n_{.1 .}=6$ & \\
\hline$\dot{j}=2$ & $n_{12 .}=5$ & $n_{22 .}=10$ & $n_{.2 .}=15$ & $n_{i j .}=\frac{n_{i . .} n_{. j .}}{n}$, \\
\hline$n_{i .}$ & $n_{1 . .}=7$ & $n_{2 . .}=14$ & $n_{\ldots}=21$ & $\begin{array}{l}\therefore C\left(X_{\alpha \alpha_{u}}\right) \\
-\end{array}$ \\
\hline
\end{tabular}

ii) Ignorando o fator $B$

\begin{tabular}{|c|c|c|c|c|}
\hline \multirow[b]{2}{*}{ Fator C } & \multicolumn{2}{|c|}{ Fator $A$} & \multirow[b]{2}{*}{$n_{. . k}$} & $n_{i . k}-\frac{n_{i . k^{\prime}}}{-4}$ \\
\hline & $i=1$ & $i=2$ & & $=\overline{3}$ ou $\overline{2}$ \\
\hline$k=1$ & $n_{1.1}=4$ & $n_{2.1}=8$ & $n_{. .1}=12$ & \\
\hline$k=2$ & $n_{1.2}=3$ & $n_{2.2}=6$ & $n_{. .2}=9$ & $n_{i . k}=\frac{n_{i . .} n_{. . k}}{n}, \quad \forall(i, k)$ \\
\hline$n_{i .}$ & $n_{1 . .}=7$ & $n_{2 . .}=14$ & $n_{\ldots}=21$ & $\therefore C\left(X_{\alpha \mid \mu}\right)$ \\
\hline
\end{tabular}

iii) Ignorando o fator $A$

\begin{tabular}{|c|c|c|c|c|}
\hline \multirow[b]{2}{*}{ Fator C } & \multicolumn{2}{|c|}{ Fator $B$} & \multirow[b]{2}{*}{$n_{. . k}$} & \multirow{2}{*}{$\frac{n_{\cdot j k}}{n_{\cdot j^{\prime} k}} \neq \frac{n_{\cdot j k^{\prime}}}{n_{\cdot j^{\prime} k^{\prime}}}$} \\
\hline & $j=1$ & $j=2$ & & \\
\hline$k=1$ & $n_{.11}=3$ & $n_{.21}=9$ & $n_{. .1}=12$ & $n_{. j .} n_{. . k}$ \\
\hline$k=2$ & $n_{.12}=3$ & $n_{.22}=6$ & $n_{.2}=9$ & $n_{. j k} \neq-$ \\
\hline$n_{. j}$ & $n_{.1 .}=6$ & $n_{.2 .}=15$ & $n_{\ldots}=21$ & $\therefore C\left(X_{\beta \mid \mu}\right) \not \subset C\left(X_{\gamma \mid \mu}\right)$ \\
\hline
\end{tabular}


Uma observaçāo pouco cuidadosa das freqüências poderia definir o delineamento como não ortogonal, em virtude da desigualdade entre a proporcionalidade das freqüências correspondentes aos fatores $B$ e $C$. Entretanto, as freqüências do fator $A$ são individualmente ortogonais às freqüências dos fatores $B$ e $C$ caracterizando assim, o delineamento como. parcialmente ortogonal. As implicações desse resultado nos efeitos principais de modelos sem interações e nas interações estão detalhadas nos itens seguintes.

\section{a Modelo sem interações}

A forma matricial do modelo $y=X \theta+e$ caracterizado por $y_{i j k l}=\mu+\alpha_{i}+\beta_{j}+\gamma_{k}+e_{i j k l}$, para os dados da Tabela 20 é dada por:

\begin{tabular}{|c|c|c|c|}
\hline 12] & {$\left[\begin{array}{l:llllll}1 & 1 & 0 & 1 & 0 & 1 & 0\end{array}\right]$} & & $e_{1111}$ \\
\hline 28 & $\begin{array}{lll:llll}1 & 1 & 0 & 1 & 0 & 0 & 1\end{array}$ & & $e_{1121}$ \\
\hline 38 & $\begin{array}{l:llllll}1 & 1 & 0 & 1 & 1 & 0\end{array}$ & & $e_{1211}$ \\
\hline 46 & $1: 10: 0 \begin{array}{lllllll} & 1 & 0 & 1 & 0\end{array}$ & & $e_{1212}$ \\
\hline 42 & $1: 10: 01: 10$ & & $e_{1213}$ \\
\hline 40 & $\begin{array}{l:lllllll}1 & 1 & 0 & 0 & 1 & 0 & 1\end{array}$ & & $e_{1221}$ \\
\hline 46 & $1: 1: 001: 01$ & & $e_{1222}$ \\
\hline 30 & $1: 01: 0 \begin{array}{lllll}0 & 0 & 1\end{array}$ & $\underline{\mu}$ & $e_{2111}$ \\
\hline 22 & $1: 0 \begin{array}{llllllll} & 1 & 0 & 1 & 0\end{array}$ & $\alpha_{1}$ & $e_{2112}$ \\
\hline 34 & $\begin{array}{l:ll:llll}1 & 0 & 1 & 1 & 0 & 1 & 0\end{array}$ & $\alpha_{2}$ & $e_{2121}$ \\
\hline 26 & $=\left[\begin{array}{lll:llll}1 & 0 & 1 & 1 & 0 & 0 & 1\end{array}\right.$ & $\bar{\beta}_{1}$ & $e_{2122}$ \\
\hline 12 & $1: 0 \begin{array}{lllllllllll}1 & 1 & 0 & 1\end{array}$ & $\underline{\beta}_{2}$ & $e_{2211}$ \\
\hline 22 & $1: 0 \begin{array}{lllll}0 & 0 & 1 & 1 & 0\end{array}$ & $\gamma_{1}$ & $e_{2212}$ \\
\hline 30 & 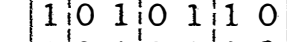 & $\gamma_{2}$ & $e_{2213}$ \\
\hline 12 & $1: 01: 01: 10$ & & $e_{2214}$ \\
\hline 18 & $1: 0 \begin{array}{llllll}0 & 0 & 1 & 1 & 0\end{array}$ & & $e_{2215}$ \\
\hline 38 & $1: 0 \begin{array}{lllllllll} & 0 & 0 & 1 & 1 & 0\end{array}$ & & $e_{2216}$ \\
\hline 58 & $1: 0 \begin{array}{lllll}0 & 0 & 1 & 1 & 0\end{array}$ & & $e_{2211}$ \\
\hline 56 & $1: 0 \begin{array}{lll:llll} & 0 & 1 & 0 & 1\end{array}$ & & $e_{2222}$ \\
\hline 62 & $1: 01: 0 \begin{array}{lllll}0 & 0 & 1\end{array}$ & & $e_{2223}$ \\
\hline 52$]$ & {$\left[\begin{array}{lll:llll}1 & 0 & 1 & 0 & 1 & 0 & 1\end{array}\right]$} & & $e_{2224}$ \\
\hline
\end{tabular}

onde:

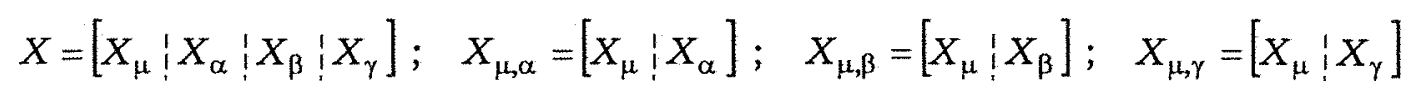

Para mostrar como a ortogonalidade parcial afeta a forma das funções estimáveis do exemplo da Tabela 20 foram formuladas hipóteses dos tipos I e II para os efeitos principais, utilizando-se o modelo de médias de 
caselas caracterizado por $y_{i j k l}=\mu_{i}+\beta_{j}+\gamma_{k}+e_{i j k l}$, para 0 fator $A$; $y_{i j k l}=\mu_{j}+\alpha_{i}+\gamma_{k}+e_{i j k l}$ para o fator $B$ e $y_{i j k l}=\mu_{k}+\alpha_{i}+\beta_{j}+e_{i j k l}$ para o fator $C$ (Speed, Hocking e Hackney, 1978).

\section{a. 1 Hipótese do tipo I para o fator $A$}

Procedendo-se de modo análogo aos anteriores, obteve-se:

$$
\begin{aligned}
H_{o}^{1-A}: & -\frac{\left(\mu_{1}+\beta_{1}+\gamma_{1}\right)+\left(\mu_{1}+\beta_{1}+\gamma_{2}\right)+3\left(\mu_{1}+\beta_{2}+\gamma_{1}\right)+2\left(\mu_{1}+\beta_{2}+\gamma_{2}\right)}{7}= \\
= & \frac{2\left(\mu_{2}+\beta_{1}+\gamma_{1}\right)+2\left(\mu_{2}+\beta_{1}+\gamma_{2}\right)+6\left(\mu_{2}+\beta_{2}+\gamma_{1}\right)+4\left(\mu_{2}+\beta_{2}+\gamma_{2}\right)}{14}
\end{aligned}
$$

que com as simplificações sobre o modelo- $\mathrm{M}$ e, a parametrização e as simplificações sobre o modelo-S, resulta:

\section{a.1.1 Modelo-M}

$H_{o}^{1-A}: \mu_{1}=\mu_{2}$

\section{a.1.2 Modelo-S}

$H_{o}^{1-A}: \alpha_{1}=\alpha_{2}$

\section{a.2 Hipótese do tipo II para o fator $A$}

$H_{o}^{2-A}:-\frac{\left(\mu_{1}+\beta_{1}+\gamma_{1}\right)+\left(\mu_{1}+\beta_{1}+\gamma_{2}\right)+3\left(\mu_{1}+\beta_{2}+\gamma_{1}\right)+2\left(\mu_{1}+\beta_{2}+\gamma_{2}\right)}{7}=\frac{\bar{C}_{1}+\bar{C}_{2}+3 \bar{C}_{3}+2 \bar{C}_{4}}{7}$

onde:

$\begin{array}{ll}\bar{C}_{1}=\frac{\left(\mu_{1}+\beta_{1}+\gamma_{1}\right)+2\left(\mu_{2}+\beta_{1}+\gamma_{1}\right)}{3} ; & \bar{C}_{3}=\frac{3\left(\mu_{1}+\beta_{2}+\gamma_{1}\right)+6\left(\mu_{2}+\beta_{2}+\gamma_{1}\right)}{9} \\ \bar{C}_{2}=\frac{\left(\mu_{1}+\beta_{1}+\gamma_{2}\right)+2\left(\mu_{2}+\beta_{1}+\gamma_{2}\right)}{3} ; & \bar{C}_{4}=\frac{2\left(\mu_{1}+\beta_{2}+\gamma_{2}\right)+4\left(\mu_{2}+\beta_{2}+\gamma_{2}\right)}{6}\end{array}$

\section{a.2.1 Modelo-M}

$H_{o}^{2-A}: \mu_{1}=\mu_{2}$

\section{a.2.2 Modelo-s}

$H_{o}^{2-A}: \alpha_{1}=\alpha_{2}$ 


\section{b. 1 Hipótese do tipo I para o fator $B$}

$$
\begin{aligned}
H_{\circ}^{1-B}: & : \frac{\left(\mu_{1}+\alpha_{1}+\gamma_{1}\right)+\left(\mu_{1}+\alpha_{1}+\gamma_{2}\right)+2\left(\mu_{1}+\alpha_{2}+\gamma_{1}\right)+2\left(\mu_{1}+\alpha_{2}+\gamma_{2}\right)}{6}= \\
& =\frac{3\left(\mu_{2}+\alpha_{1}+\gamma_{1}\right)+2\left(\mu_{2}+\alpha_{1}+\gamma_{2}\right)+6\left(\mu_{2}+\alpha_{2}+\gamma_{1}\right)+4\left(\mu_{2}+\alpha_{2}+\gamma_{2}\right)}{15}
\end{aligned}
$$

\section{b.1.1 Modelo-M}

$H_{o}^{1-B}: \mu_{1}+1 / 10 \gamma_{1}=\mu_{2}+1 / 10 \gamma_{2}$

\section{b.1.2 Modelo-S}

$H_{o}^{1-B}: \beta_{1}+1 / 10 \gamma_{1}=\beta_{2}+1 / 10 \gamma_{2}$

Observa-se para o fator $B$ que a ponderação não ajusta para o fator $C$, o que se visualiza pela presença de parâmetro $\gamma$. Esta característica comum aos delineamentos não ortogonais, como visto, ocorre para os delineamentos parcialmente ortogonais para os fatores cujas freqüências não foram proporcionais aos demais.

\section{b.2 Hipótese do tipo II para o fator B}

$H_{o}^{2-B}:-\frac{\left(\mu_{1}+\alpha_{1}+\gamma_{1}\right)+\left(\mu_{1}+\alpha_{1}+\gamma_{2}\right)+2\left(\mu_{1}+\alpha_{2}+\gamma_{1}\right)+2\left(\mu_{1}+\alpha_{2}+\gamma_{2}\right)}{6}=\frac{\bar{C}_{1}+\bar{C}_{2}+2 \bar{C}_{3}+2 \bar{C}_{4}}{6}$.

onde:

$$
\begin{array}{ll}
\bar{C}_{1}=\frac{\left(\mu_{1}+\alpha_{1}+\gamma_{1}\right)+3\left(\mu_{2}+\alpha_{1}+\gamma_{1}\right)}{4} ; & \bar{C}_{3}=\frac{2\left(\mu_{1}+\alpha_{2}+\gamma_{1}\right)+6\left(\mu_{2}+\alpha_{2}+\gamma_{1}\right)}{8} \\
\bar{C}_{2}=\frac{\left(\mu_{1}+\alpha_{1}+\gamma_{2}\right)+2\left(\mu_{2}+\alpha_{1}+\gamma_{2}\right)}{3} ; & \bar{C}_{4}=\frac{2\left(\mu_{1}+\alpha_{2}+\gamma_{2}\right)+4\left(\mu_{2}+\alpha_{2}+\gamma_{2}\right)}{6}
\end{array}
$$

\section{b.2. 1 Modelo-M}

$H_{\circ}^{2-B}: \mu_{1}=\mu_{2}$

\section{b.2.2 Modelo-S}

$H_{o}^{2-B}: \beta_{1}=\beta_{2}$

\section{c. 1 Hipótese do tipo I para o fator C}

$$
\begin{aligned}
H_{o}^{1-C} & : \frac{\left(\mu_{1}+\alpha_{1}+\beta_{1}\right)+3\left(\mu_{1}+\alpha_{1}+\beta_{2}\right)+2\left(\mu_{1}+\alpha_{2}+\beta_{1}\right)+6\left(\mu_{1}+\alpha_{2}+\beta_{2}\right)}{12}= \\
& =\frac{\left(\mu_{2}+\alpha_{1}+\beta_{1}\right)+2\left(\mu_{2}+\alpha_{1}+\beta_{2}\right)+2\left(\mu_{2}+\alpha_{2}+\beta_{1}\right)+4\left(\mu_{2}+\beta_{2}+\gamma_{2}\right)}{9}
\end{aligned}
$$


c.1.1 Modelo-M

$H_{o}^{1-C}: \gamma_{1}+1 / 12 \beta_{1}=\gamma_{2}+1 / 12 \beta_{2}$ c.1.2 Modelo-S

$H_{o}^{1-C}: \gamma_{1}+1 / 12 \beta_{1}=\gamma_{2}+1 / 12 \beta_{2}$

\section{c.2 Hipótese do tipo II para o fator C}

$H_{o}^{2-C}: \frac{\left(\mu_{1}+\beta_{1}+\gamma_{1}\right)+3\left(\mu_{1}+\alpha_{1}+\beta_{2}\right)+2\left(\mu_{1}+\alpha_{2}+\beta_{1}\right)+6\left(\mu_{1}+\alpha_{2}+\beta_{2}\right)}{12}=\frac{\bar{C}_{1}+3 \bar{C}_{2}+2 \bar{C}_{3}+6 \bar{C}_{4}}{12}$

onde:

$\begin{array}{ll}\bar{C}_{1}=\frac{\left(\mu_{1}+\alpha_{1}+\beta_{1}\right)+\left(\mu_{2}+\alpha_{1}+\beta_{1}\right)}{2} ; & \bar{C}_{3}=\frac{2\left(\mu_{1}+\alpha_{2}+\beta_{1}\right)+2\left(\mu_{2}+\alpha_{2}+\beta_{1}\right)}{4} \\ \bar{C}_{2}=\frac{3\left(\mu_{1}+\alpha_{1}+\beta_{2}\right)+2\left(\mu_{2}+\alpha_{1}+\beta_{2}\right)}{5} ; & \bar{C}_{4}=\frac{6\left(\mu_{1}+\alpha_{2}+\beta_{2}\right)+4\left(\mu_{2}+\alpha_{2}+\beta_{2}\right)}{10}\end{array}$

\section{c.2.1 Modelo-M}

$H_{o}^{2-C}: \mu_{1}=\mu_{2}$

\section{c.2.2 Modelo-S}

$H_{0}^{2-C}: \gamma_{1}=\gamma_{2}$

Os resultados dos itens a.1.2 e a.2.2 evidenciam a igualdade entre as hipóteses dos tipos I e II para o fator $A$, decorrente da ortogonalidade $\mathrm{e}$, consequentemente, a ausência de parâmetros dos fatores $B$ e $C$ nas funções estimáveis, apresentando apenas parâmetros do próprio fator $A$. Ainda, como foi detectado que as freqüências de caselas entre os fatores $B$ e $C$ não são proporcionais não ocorreu, portanto, a igualdade entre as hipóteses dos tipos I e II para o fator $B$ (b.1.2 e b.2.2) e para o fator $C$ (c.1.2 e c.2.2).

A forma da função estimável do tipo I para o fator $B$ (b.1.1 ou b.1.2) revelou a presença de parâmetros do fator $C$, pela não ortogonalidade entre os subespaços gerados entre os dois fatores, e a ausência de parâmetros do fator $A$ devida à ortogonalidade dos subespaços gerados pelos fatores $A$ e $B$ $\mathrm{e}$, para o fator $C$ (c.1.1 ou c.1.2) as funções estimáveis revelaram parâmetros do fator $B$ e ausência de parâmetros de $A$. Todos esses resultados podem ser observados no Quadro 16. 
Quadro 16. Funçōes estimáveis fornecidas pelo Proc GLM do SAS e respectivas hipóteses sobre os fatores $A, B$ e $C$ para os dados da Tabela 20, considerando o modelo sem interações.

\begin{tabular}{|c|c|c|c|c|}
\hline \multicolumn{3}{|c|}{ Tipo $I-A=$ Tipo II-A } & \multicolumn{2}{|c|}{ Tipo II-A = Tipo IV-A } \\
\hline \multicolumn{2}{|c|}{$\begin{array}{l}\text { EFeito } \\
\text { INTERCEPTO }\end{array}$} & $\begin{array}{l}\text { Coeficientes } \\
0\end{array}$ & $\begin{array}{l}\text { Efeito } \\
\text { INTERCEPTO }\end{array}$ & $\begin{array}{l}\text { Coeficientes } \\
0\end{array}$ \\
\hline A & $\begin{array}{l}1 \\
2\end{array}$ & $\begin{array}{l}\mathrm{L} 2 \\
-\mathrm{L} 2\end{array}$ & & $\begin{array}{l}\mathrm{L} 2 \\
-\mathrm{L} 2\end{array}$ \\
\hline B & $\begin{array}{l}1 \\
2\end{array}$ & $: 0$ & B & 0 \\
\hline c & $\frac{1}{2}$ & $\begin{array}{l}0 \\
0\end{array}$ & $c$ & $: 0$ \\
\hline \multicolumn{5}{|c|}{$H_{o}^{1-A}=H_{o}^{2-A}=H_{o}^{3-A}=H_{o}^{4-A}: \alpha_{1}=\alpha_{2}$} \\
\hline \multicolumn{3}{|c|}{ Tipo I-B } & \multicolumn{2}{|c|}{ Tipo II-B $=$ Tipo III-B $=$ Tipo IV-B } \\
\hline \multicolumn{2}{|c|}{$\begin{array}{l}\text { Efeito } \\
\text { INTERCEPTO }\end{array}$} & ${ }_{0}^{\text {Coficientes }}$ & $\begin{array}{l}\text { EEeito } \\
\text { INTERCEPTO }\end{array}$ & $\begin{array}{l}\text { Coeficientes } \\
0\end{array}$ \\
\hline B & $\frac{1}{2}$ & $\begin{array}{l}\mathrm{L} 2 \\
-\mathrm{L} 2\end{array}$ & B & $\begin{array}{l}\mathrm{L} 2 \\
-\mathrm{L} 2\end{array}$ \\
\hline A & $\frac{1}{2}$ & $:$ & A & $\begin{array}{l}0 \\
0\end{array}$ \\
\hline c & $\begin{array}{l}1 \\
2\end{array}$ & $\begin{array}{l}-0,1 * \mathrm{~L} 2 \\
0,1 * \mathrm{~L} 2\end{array}$ & $\mathrm{C}$ & $\begin{array}{l}0 \\
0\end{array}$ \\
\hline \multicolumn{3}{|c|}{$H_{o}^{1-B}: \beta_{1}+1 / 10 \gamma_{1}=\beta_{2}+1 / 10 \gamma_{2}$} & \multicolumn{2}{|c|}{$H_{o}^{2-B}=H_{o}^{3-B}=H_{o}^{4-B}: \beta_{1}=\beta_{2}$} \\
\hline \multicolumn{3}{|c|}{ Tipo I-C } & \multicolumn{2}{|c|}{ Tipo $I-C=T i p o I I-C=T i p o I V-C$} \\
\hline \multicolumn{2}{|c|}{$\begin{array}{l}\text { Efeito } \\
\text { INTERCEPTO }\end{array}$} & $\begin{array}{l}\text { Coeficientes } \\
0\end{array}$ & $\begin{array}{l}\text { Efeito } \\
\text { INTERCEPTO }\end{array}$ & $\begin{array}{l}\text { Coeficientes } \\
0\end{array}$ \\
\hline C & $\frac{1}{2}$ & $\begin{array}{l}\mathrm{L} 2 \\
-\mathrm{L} 2\end{array}$ & c & $\begin{array}{l}\mathrm{L} 2 \\
-\mathrm{L} 2\end{array}$ \\
\hline B & $\begin{array}{l}1 \\
2\end{array}$ & $\begin{array}{l}-0,0833 * \mathrm{~L} 2 \\
0,0833 * \mathrm{~L} 2\end{array}$ & B & $\begin{array}{l}0 \\
0\end{array}$ \\
\hline A & $\frac{1}{2}$ & $:$ & A & $:$ \\
\hline \multicolumn{3}{|c|}{$H_{o}^{1-C}: \gamma_{1}+1 / 12 \beta_{1}=\gamma_{2}+1 / 12 \beta_{2}$} & \multicolumn{2}{|c|}{$H_{o}^{2-C}=H_{o}^{3-C}=H_{o}^{4-C}: \gamma_{1}=\gamma_{2}$} \\
\hline
\end{tabular}

As conseqüências da ortogonalidade parcial nas somas de quadrados associadas às hipóteses do exemplo da Tabela 20, são apresentadas 
nas Tabelas 21, 22 e 23 que correspondem a mesma análise, mas com a variação da ordem de entrada dos fatores no modelo.

A Tabela 21 apresenta a análise de variância do exemplo da Tabela 20 e mostra que, com o fator $A$ sendo declarado em primeiro lugar no modelo, a soma de quadrados associada à hipótese do tipo I para o fator é igual à soma de quadrados associada à hipótese do tipo II, ou seja, $R(\alpha \mid \mu)=R(\alpha \mid \mu, \beta, \gamma)=24,3809$. Assim, $P_{\alpha \mid \mu}=P_{\alpha \mid \mu, \beta, \gamma}$ e como conseqüência, $P_{\alpha \mid \mu}=P_{\alpha \mid \mu, \beta}=P_{\alpha \mid \mu, \gamma}=P_{\alpha \mid \mu, \beta, \gamma}, \quad$ então $\quad R(\alpha \mid \mu)=R(\alpha \mid \mu, \beta)=R(\alpha \mid \mu, \gamma)=R(\alpha \mid \mu, \beta, \gamma)=$ =24,3809 (Tabelas 21, 22 e 23). Como o modelo não contém interações $R(\alpha \mid \mu, \beta, \gamma)=R(\dot{\alpha} \mid \dot{\mu}, \dot{\beta}, \dot{\gamma})=24,3809, R(\beta \mid \mu, \alpha, \gamma)=R(\dot{\beta} \mid \dot{\mu}, \dot{\alpha}, \dot{\gamma})=919,1176$ e $R(\gamma \mid \mu, \alpha, \beta)=$ $=R(\dot{\gamma} \mid \dot{\mu}, \dot{\alpha}, \dot{\beta})=1852,5176$.

Tabela 21. Análise de variância do delineamento parcialmente ortogonal, descrito na Tabela 20, segundo o modelo $y_{i j k l}=\mu+\alpha_{i}+\beta_{j}+\gamma_{k}+e_{i j k l}$, declarando A como primeiro fator

\begin{tabular}{|c|c|c|c|c|}
\hline Fontes & $g l$ & S.Q. Tipo I & Q.M. & $R()$. \\
\hline A & 1 & 24,38095238 & 24,38095238 & $R(\alpha \mid \mu)$ \\
\hline B & 1 & 702,17142857 & 702,17142857 & $R(\beta \mid \mu, \alpha)$ \\
\hline C & 1 & 1852,51764706 & 1852,51764706 & $R(\gamma \mid \mu, \alpha, \beta)$ \\
\hline Fontes & $g l$ & S.Q. Tipo II & $Q . M$. & $R(\cdot)$ \\
\hline A & 1 & 24,38095238 & 24,38095238 & $R(\alpha \mid \mu, \beta, \gamma)$ \\
\hline B & i & 919,11764706 & 919,11764706 & $R(\beta \mid \mu, \alpha, \gamma)$ \\
\hline c & 1 & 1852,51764706 & 1852,51764706 & $R(\gamma \mid \mu, \alpha, \beta)$ \\
\hline Fontes & $g l$ & $S Q$ Tipo $I I I=I V$ & Q.M. & $R()$. \\
\hline $\mathbf{A}$ & 1 & 24,38095238 & 24,38095238 & $\boldsymbol{R}(\dot{\alpha} \mid \dot{\mu}, \dot{\beta}, \dot{\gamma})$ \\
\hline B & 1 & 919,11764706 & 919,11764706 & $R(\dot{\beta} \mid \dot{\mu}, \dot{\alpha}, \dot{\gamma})$ \\
\hline c & 1 & 1852,51764706 & 1852,51764706 & $R(\dot{\gamma} \mid \dot{\mu}, \dot{\alpha}, \dot{\beta})$ \\
\hline
\end{tabular}

Como as freqüências entre os fatores $B \cdot e$ não são proporcionais, a soma de quadrados associada à hipótese do tipo I para o fator $B$ não é igual a soma de quadrados associada à hipótese do tipo II, ou seja, $R(\beta \mid \mu) \neq R(\beta \mid \mu, \alpha, \gamma)$ (Tabela 22). Entretanto, essa desigualdade não permite inferir que todas as decomposiçōes de somas de quadrados para o fator $B$ 
ajustado para constante $\mu$ são diferentes das demais decomposições. No exemplo, a ortogonalidade entre $A$ e $B$ fez com que a soma de quadrados para o fator $B$ ajustado para $\mu$ fosse igual a soma de quadrados ajustado para $\mu$ e para o fator $A$, ou seja, $R(\beta \mid \mu)=R(\beta \mid \mu, \alpha)=702,1714$ (Tabelas 21 e 22) e portanto, $P_{\beta \mid \mu}=P_{\beta \mid \mu, \alpha}$. Os resultados mostram que a desigualdade entre as hipóteses dos tipos I e II, para os efeitos principais de modelos com mais de dois fatores não implica na desigualdade de todos os projetores referentes aos ajustamentos.

Tabela 22. Análise de variância do delineamento parcialmente ortogonal, descrito na Tabela 20, segundo o modelo $y_{i j k l}=\mu+\beta_{j}+\alpha_{i}+\gamma_{k}+e_{i j k l}$, declarando $B$ como primeiro fator.

\begin{tabular}{lcrrl}
\hline Fontes & $g l$ & S.Q. Tipo I & Q.M. & $R(\cdot)$ \\
\hline B & 1 & 702,17142857 & 702,17142857 & $\boldsymbol{R}(\beta \mid \mu)$ \\
A & 1 & 24,38095238 & 24,38095238 & $R(\alpha \mid \mu, \beta)$ \\
C & 1 & 1852,51764706 & 1852,51764706 & $R(\gamma \mid \mu, \alpha, \beta)$ \\
\hline Fontes & $g l$ & S.Q. Tipo II & $Q . M$ & $R(\cdot)$ \\
\hline B & 1 & 919,11764706 & 313,11754706 & $\boldsymbol{R}(\beta \mid \mu, \alpha, \gamma)$ \\
A & 1 & 24,38095238 & 24,38095238 & $R(\alpha \mid \mu, \beta, \gamma)$ \\
C & 1 & 1852,51764706 & 1852,51764706 & $R(\gamma \mid \mu, \alpha, \beta)$ \\
\hline Fontes & $g l$ & S.Q. Tipo III=IV & $Q . M$. & $R(\cdot)$ \\
\hline B & 1 & 919,11764706 & 919,11754706 & $\boldsymbol{R}(\dot{\beta} \mid \dot{\mu}, \dot{\alpha}, \dot{\gamma})$ \\
A & 1 & 24,38095238 & 24,38095238 & $R(\dot{\alpha} \mid \dot{\mu}, \dot{\beta}, \dot{\gamma})$ \\
C & 1 & 1852,51764706 & 1852,51764706 & $R(\dot{\gamma} \mid \dot{\mu}, \dot{\alpha}, \dot{\beta})$ \\
\hline
\end{tabular}

Da mesma forma que ocorrido para o fator $B$, a soma de quadrados associada à hipótese do tipo I para o fator $C$ é diferente da soma de quadrados associada à hipótese do tipo II, $R(\gamma \mid \mu) \neq R(\gamma \mid \mu, \alpha, \beta)$, como apresentou a Tabela 23. No entanto, $R(\gamma \mid \mu)=R(\gamma \mid \mu, \alpha)=1635,5714$ e, portanto, $P_{\gamma \mid \mu}=P_{\gamma \mid \mu, \alpha}$. 
Tabela 23. Análise de variância do delineamento parcialmente ortogonal, descrito na Tabela 20, segundo o modelo $y_{i j k l}=\mu+\gamma_{k}+\alpha_{i}+\beta_{j}+e_{i j k l}$, declarando $C$ como primeiro fator.

\begin{tabular}{|c|c|c|c|c|}
\hline Fontes & $g l$ & S.Q. Tipo I & Q.M. & $R()$. \\
\hline c & 1 & 1635,57142857 & $16.35,571428.57$ & $R(y \nmid \mu)$ \\
\hline A & 1 & 24,36095238 & 24,36095238 & $R(\alpha \mid \mu, \gamma)$ \\
\hline B & 1 & 919,11764706 & 919,11764706 & $R(\beta \mid \mu, \alpha, \gamma)$ \\
\hline Fontes & $g l$ & S.Q. Tipo II & Q.M. & $R()$. \\
\hline$c$ & 1 & 1852,51764706 & 1852,51764706 & $R(\gamma \mid \mu, \alpha, \beta)$ \\
\hline A & 1 & 24,38095238 & 24,38095238 & $R(\propto \nmid \mu, \beta, \gamma)$ \\
\hline B & 1 & 919,11764706 & 919,11764706 & $R(\beta \mid \mu, \alpha, \gamma)$ \\
\hline Fontes & $g l$ & S.Q. Tipo $I I I=I V$ & Q.M. & $R()$. \\
\hline c & 1 & 1852,51764706 & 1052,51764706 & $R(\dot{\gamma} \mid \dot{\mu}, \dot{\alpha}, \dot{\beta})$ \\
\hline A & 1 & 24,38095238 & 24,38095238 & $\boldsymbol{R}(\dot{\alpha} \mid \dot{\mu}, \dot{\beta}, \dot{\gamma})$ \\
\hline B & 1 & 919,11764706 & 919,11764706 & $\boldsymbol{R}(\dot{\beta} \mid \dot{\mu}, \dot{\alpha}, \dot{\gamma})$ \\
\hline
\end{tabular}

\section{b Modelos com interações}

Resultados ainda mais reveladores sobre a ortogonalidade parcial, ocorrem quando o modelo contém interações. A forma das funções estimáveis para os efeitos principais e para as interações está condicionada à combinação ou às combinações entre os fatores que apresentaram. freqüências de caselas proporcionais. Como no exemplo da Tabela 20 apenas o fator $A$ apresenta freqüências proporcionais aos demais fatores, as funções estimáveis de $A$ contêm parâmetros do próprio fator $(\alpha)$ e parâmetros das interações onde o fator esteve presente; interações $A^{*} B, A^{*} C$ e $A^{*} B^{*} C$, como se observa no Quadro 17. Entretanto, as funções estimáveis do tipo I para o fator $B$ (Quadro 18) apresentam, alẻm de parâmetros do próprio fator, $(\beta)$, e do fator $C,(\gamma)$, parâmetros de todas as interações. Da mesma forma ocorre para o fator $C$ que, dada a não ortogonalidade $\operatorname{com} B$, apresenta as funções estimáveis do tipo I com parâmetros do próprio fator, parâmetros do fator $B$ e parâmetros de todas as interações (Quadro 19).

Nos Quadros 20, 21 e 22 encontram-se os resultados das funções estimáveis dos tipos I e II para todas as interações de segunda ordem. Como se 
pode observar, a única interação que apresenta funções estimáveis do tipo I iguais às funções do tipo II é a interação $B^{*} C$ (Quadro 22), justamente a interação formada pelos fatores que nāo apresentam freqüências proporcionais entre si, apresentando parâmetros da própria interação, $(\tau)$, e parâmetros da interação de maior grau, $(\rho)$. Como conseqüência, as funções estimáveis do tipo I para as interações $A{ }^{*} B$ (Quadro 20) e $A^{*} C$ (Quadro 21) só não apresentaram parâmetros dos efeitos principais e da interação $B^{*} C$.

Quadro 17. Funções estimáveis fornecidas pelo Proc GLM do SAS e respectivas hipóteses sobre o fator $A$, para os dados da Tabela 20 , considerando o modelo com interações.

\begin{tabular}{|c|c|c|c|c|c|}
\hline$T i p$ & $I-A=$ & Tipo II-A & Tip & $\boldsymbol{I I}-\mathbf{A}=$ & Tipo IV-A \\
\hline $\begin{array}{l}\text { Efeit } \\
\text { INTER }\end{array}$ & & $\begin{array}{l}\text { Coeficientes } \\
0\end{array}$ & $\begin{array}{l}\text { Efeitc } \\
\text { INTRRR }\end{array}$ & & $\begin{array}{l}\text { Coeficientes } \\
0\end{array}$ \\
\hline A & $\begin{array}{l}1 \\
2\end{array}$ & $\begin{array}{l}\mathrm{L} 2 \\
-\mathrm{L} 2\end{array}$ & A & $\begin{array}{l}1 \\
2\end{array}$ & $\begin{array}{l}\mathrm{L} 2 \\
-\mathrm{L} 2\end{array}$ \\
\hline B & $\begin{array}{l}1 \\
2\end{array}$ & $\begin{array}{l}0 \\
0\end{array}$ & $B$ & $\begin{array}{l}1 \\
2\end{array}$ & $\begin{array}{l}0 \\
0\end{array}$ \\
\hline C. & $\begin{array}{l}1 \\
2\end{array}$ & $\begin{array}{l}0 \\
0\end{array}$ & c & $\begin{array}{l}1 \\
2\end{array}$ & $\begin{array}{l}0 \\
0\end{array}$ \\
\hline$A \star B$ & $\begin{array}{ll}1 & 1 \\
1 & 2 \\
2 & 1 \\
2 & 2\end{array}$ & $\begin{array}{l}0,2857 * \mathrm{~L} 2 \\
0,7143 * \mathrm{~L} 2 \\
-0,2857 * \mathrm{~L} 2 \\
-0,7143 * \mathrm{~L} 2\end{array}$ & $A * B$ & $\begin{array}{ll}1 & 1 \\
1 & 2 \\
2 & 1 \\
2 & 2\end{array}$ & $\begin{array}{l}0,5 * \mathrm{~L} 2 \\
0,5 * \mathrm{~L} 2 \\
-0,5 * \mathrm{~L} 2 \\
-0,5 * \mathrm{~L} 2\end{array}$ \\
\hline$A * C$ & $\begin{array}{ll}1 & 1 \\
1 & 2 \\
2 & 1 \\
2 & 2\end{array}$ & $\begin{array}{l}0,5714 * \mathrm{~L} 2 \\
0,4266 * \mathrm{~L} 2 \\
-0,5714 * \mathrm{~L} 2 \\
-0,4286 * \mathrm{~L} 2\end{array}$ & $A^{\star} \mathrm{C}$ & $\begin{array}{ll}1 & 1 \\
1 & 2 \\
2 & 1 \\
2 & 2\end{array}$ & $\begin{array}{l}0,5 * L 2 \\
0,5 * I 2 \\
-0,5 * L 2 \\
-0,5 * L 2\end{array}$ \\
\hline$B^{\star} C$ & $\begin{array}{ll}1 & 1 \\
1 & 2 \\
2 & 1 \\
2 & 2\end{array}$ & $\begin{array}{l}0 \\
0 \\
0 \\
0\end{array}$ & $B+C$ & $\begin{array}{ll}1 & 1 \\
1 & 2 \\
2 & 1 \\
2 & 2\end{array}$ & $\begin{array}{l}0 \\
0 \\
0 \\
0\end{array}$ \\
\hline$A * B * C$ & $\begin{array}{lll}1 & 1 & 1 \\
1 & 1 & 2 \\
1 & 2 & 1 \\
1 & 2 & 2 \\
2 & 1 & 1 \\
2 & 1 & 2 \\
2 & 2 & 1 \\
2 & 2 & 2\end{array}$ & $\begin{array}{l}0,1429 * \mathrm{~L} 2 \\
0,1429 * \mathrm{~L} 2 \\
0,4286 * \mathrm{~L} 2 \\
0,2857 * \mathrm{~L} 2 \\
-0,1429 * \mathrm{~L} 2 \\
-0,1429 * \mathrm{~L} 2 \\
-0,4286 * \mathrm{~L} 2 \\
-0,2857 * \mathrm{~L} 2\end{array}$ & $A * B * C$ & $\begin{array}{lll}1 & 1 & 1 \\
1 & 1 & 2 \\
1 & 2 & 1 \\
1 & 2 & 2 \\
2 & 1 & 1 \\
2 & 1 & 2 \\
2 & 2 & 1 \\
2 & 2 & 2\end{array}$ & $\begin{array}{l}0,25 * \mathrm{~L} 2 \\
0,25 * \mathrm{~L} 2 \\
0,25 * \mathrm{~L} 2 \\
0,25 * \mathrm{~L} 2 \\
-0,25 * \mathrm{~L} 2 \\
-0,25 * \mathrm{~L} 2 \\
-0,25 * \mathrm{~L} 2 \\
-0,25 * \mathrm{~L} 2\end{array}$ \\
\hline $\begin{array}{r}H_{o}^{1-A}=H_{o}^{2-A}: \\
+3 \eta \\
=\alpha_{2} \\
+\rho_{2}\end{array}$ & $\begin{array}{l}1 / 7\left(2 \varphi_{1}\right. \\
-\rho_{111}+ \\
7\left(2 \varphi_{21}\right. \\
\rho_{212}+3\end{array}$ & $\begin{array}{l}+5 \varphi_{12}+4 \eta_{11}+ \\
\left.12+3 p_{121}+2 p_{122}\right)= \\
5 \varphi_{22}+4 \eta_{21}+3 \eta_{22^{\prime}}+ \\
\left.221+2 \rho_{222}\right)\end{array}$ & $\begin{array}{r}H_{0}^{3-A}=H_{o}^{4-A}: \\
+2 \gamma \\
=\alpha \\
+\rho_{2}\end{array}$ & $\begin{array}{l}+1 / 4(2 \varphi \\
+\rho_{111}+ \\
1 / 4\left(2 \varphi_{21}\right. \\
+\rho_{212}+\end{array}$ & $\begin{array}{l}1+2 \varphi_{12}+2 \eta_{11}+ \\
\left.112+\rho_{121}+\rho_{122}\right)= \\
+2 \varphi_{22}+2 \eta_{21}+2 \eta_{12}+ \\
\left.221+\rho_{222}\right)\end{array}$ \\
\hline
\end{tabular}




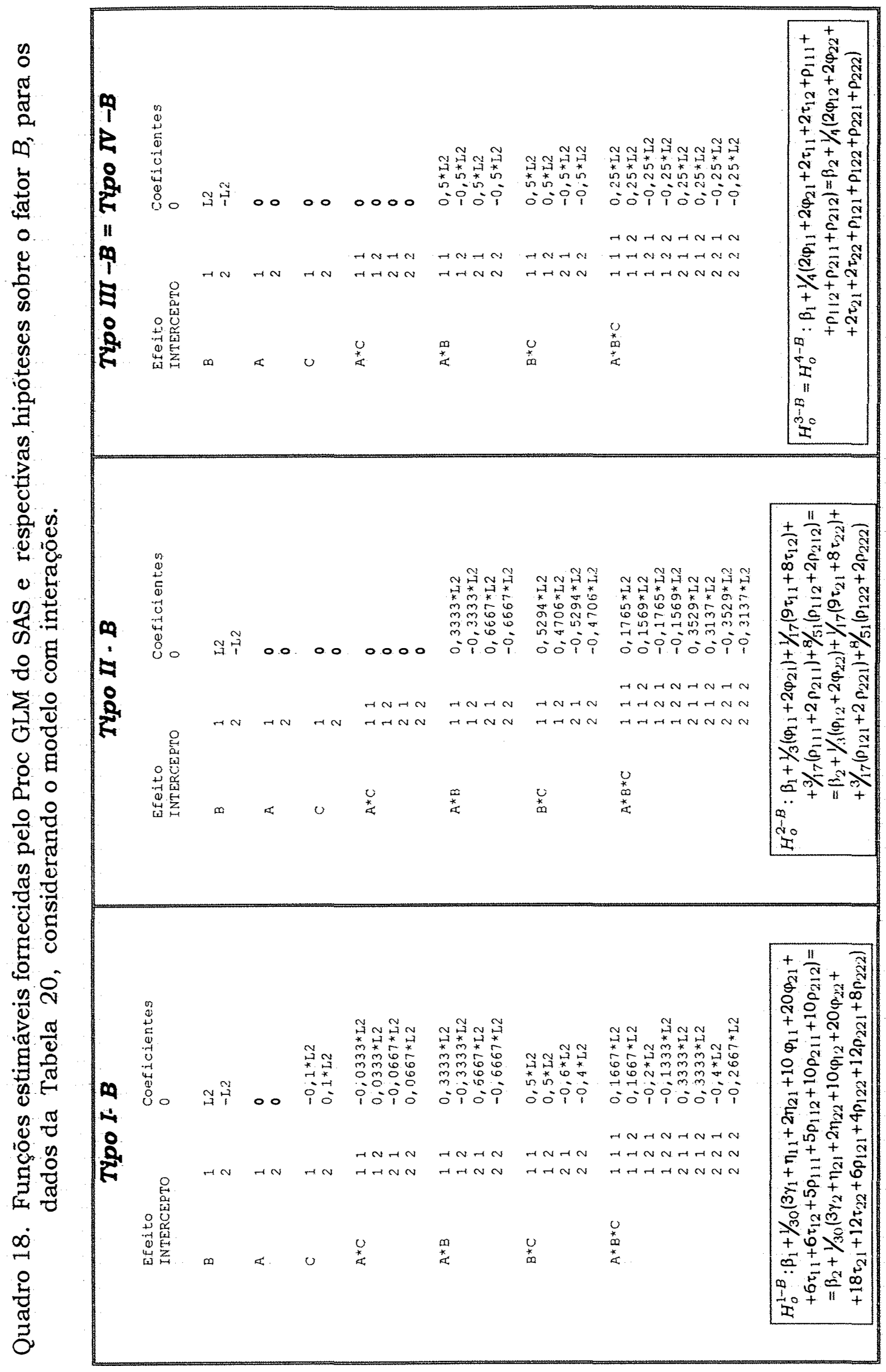




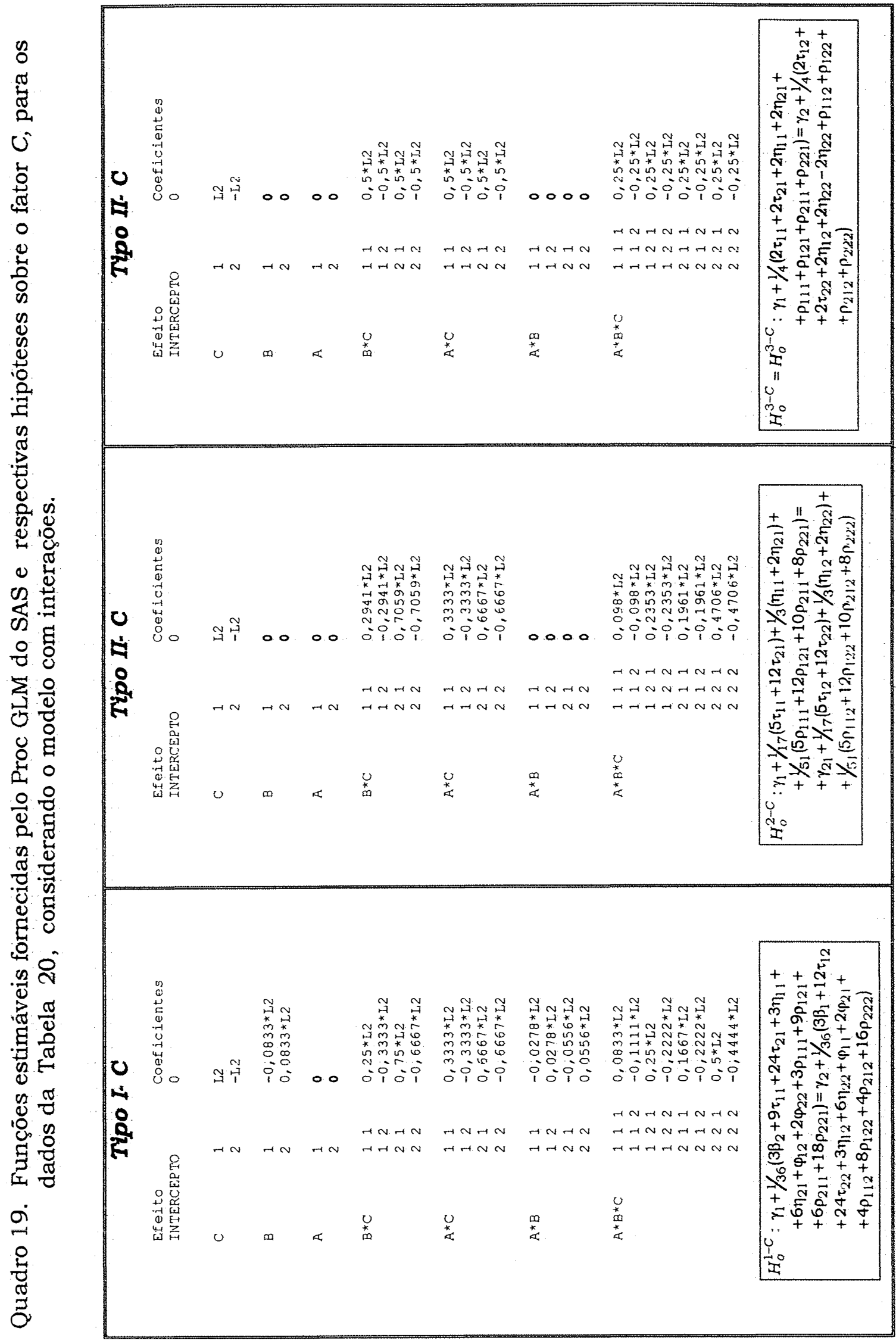




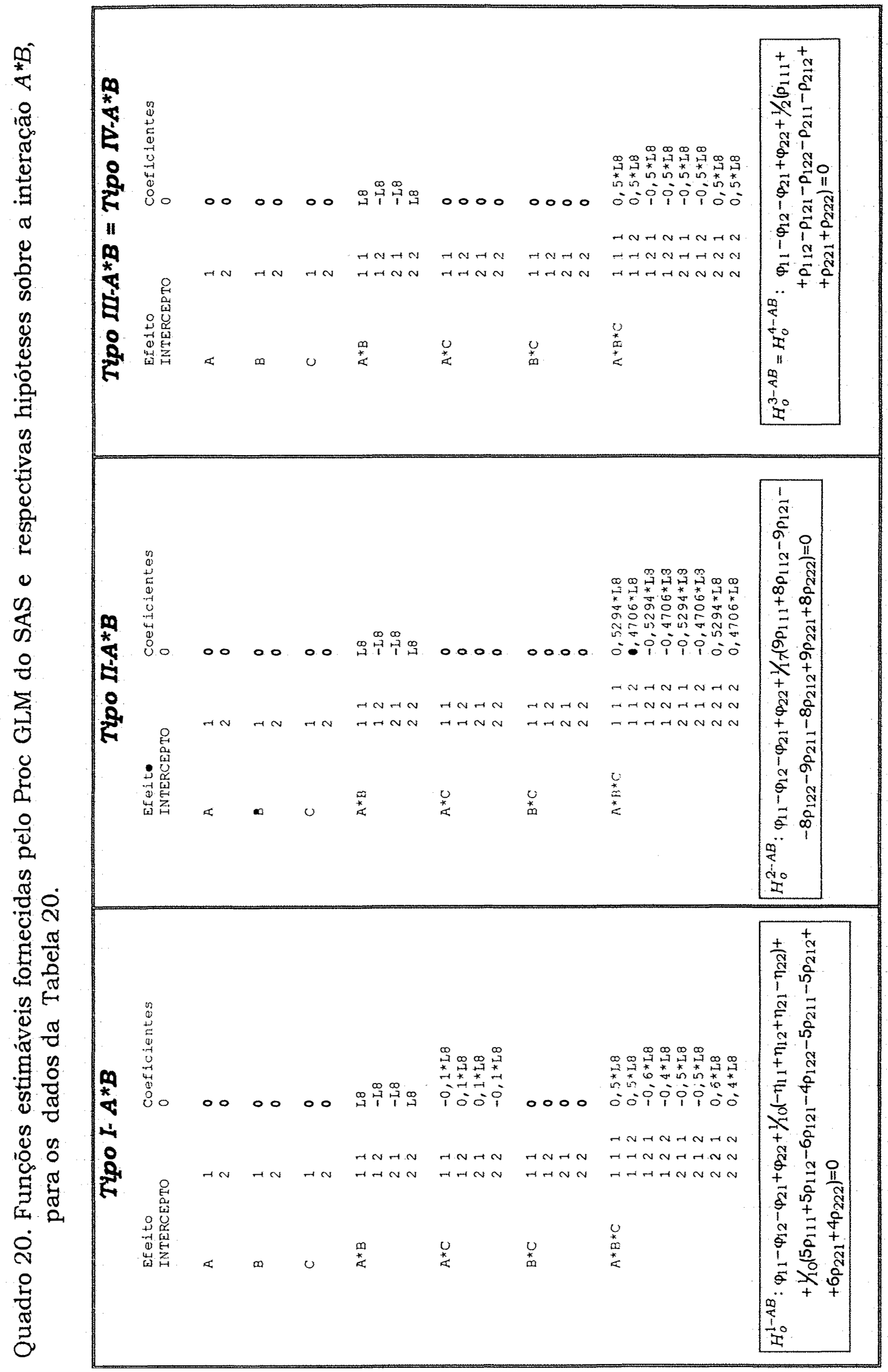




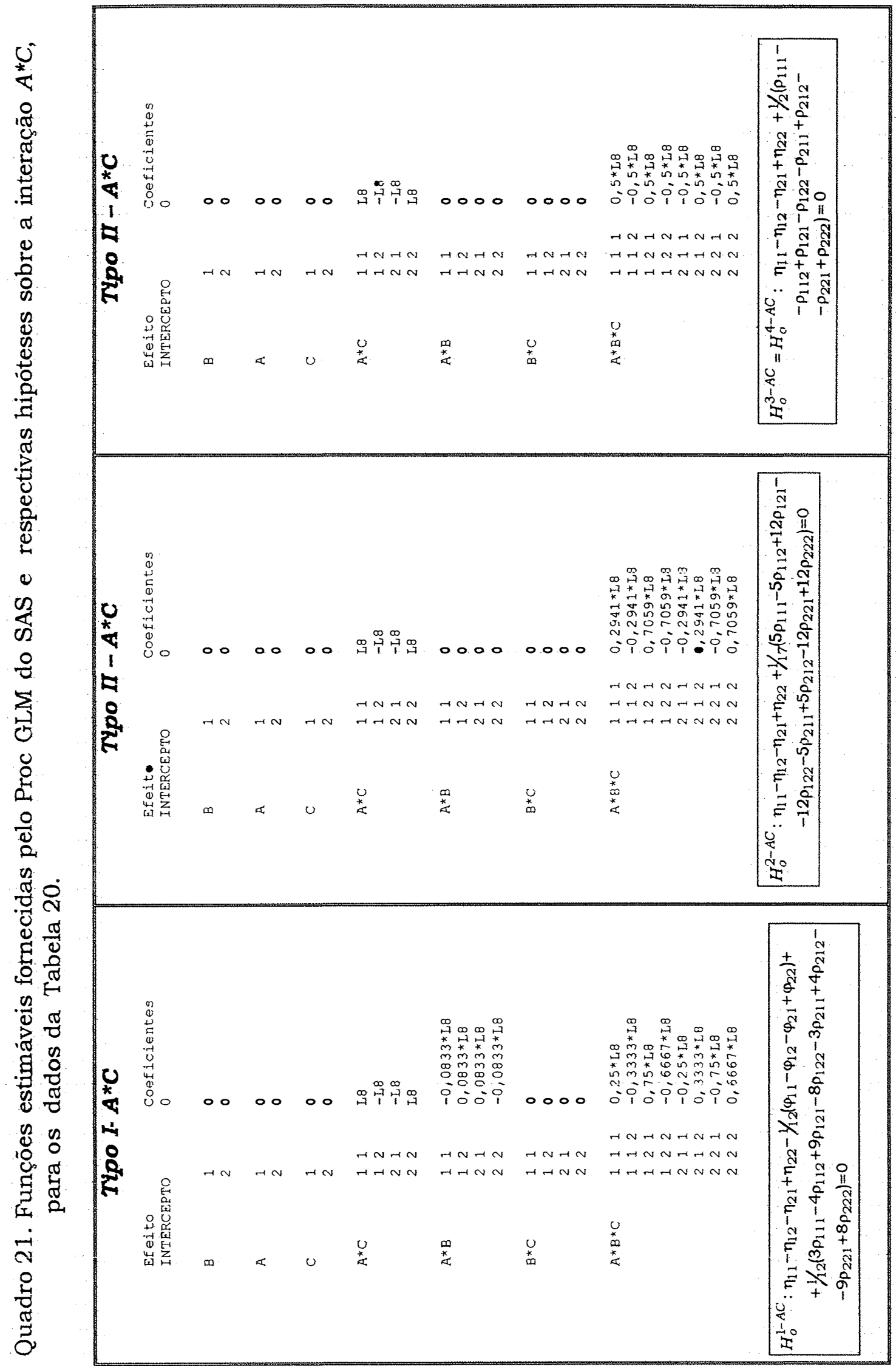




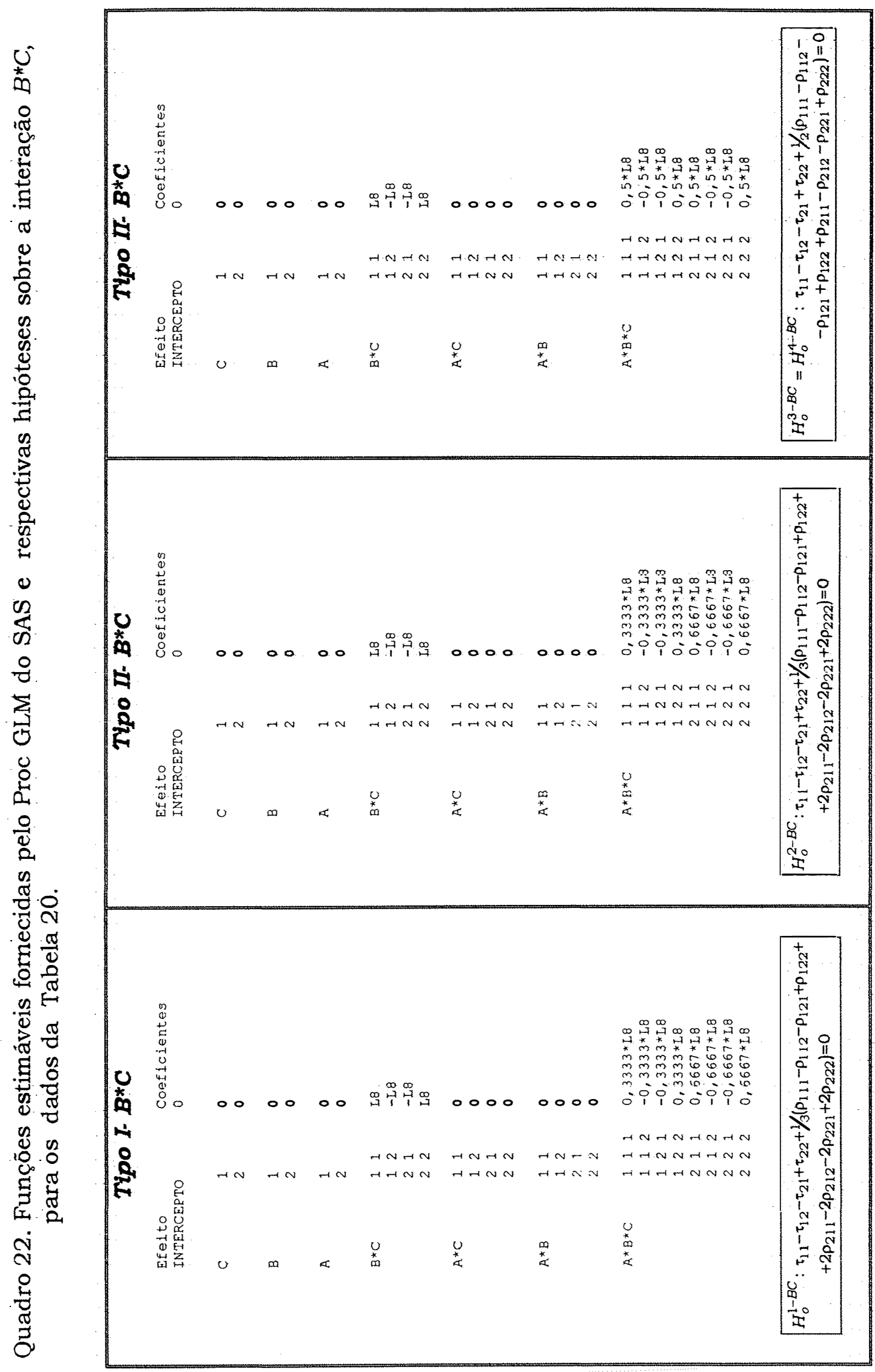


Para os efeitos principais, os resultados quanto à igualdade de hipóteses no modelo com interaçōes são idênticos aos ocorridos nos modelos sem interações, ou seja, apenas para o fator $A$ a soma de quadrados associada à hipótese do tipo I $A$ é igual à soma de quadrados associada à hipótese do tipo II, como mostra a Tabela 24. Como várias.vezes mencionado neste texto, o Proc GLM do SAS apenas fornece hipóteses do tipo I e conseqüente soma de quadrados associada, se o fator ou a interação de interesse forem declarados em primeiro lugar no modelo.

Ainda na Tabela 24 tem-se a interação $A * B$ declarada em primeiro lugar, comprovando a desigualdade entre as somas de quadrados associadas as hipóteses dos tipos I e II, portanto, $R(\varphi \mid \mu, \alpha, \beta, \gamma) \neq R(\varphi \mid \mu, \alpha, \beta, \gamma, \eta, \tau)$. A ordem de entrada dos parâmetros no modelo é importante para que o SAS forneça uma soma de quadrados associada ao tipo I, na qual tem-se uma soma de quadrados ajustada apenas para a constante $\mu$ e para os efeitos principais, ou seja, $R(\varphi \mid \mu, \alpha, \beta, \gamma)$. As interaçōes que não foram declaradas em primeiro lugar foram ajustadas, além da constante e dos efeitos principais, pelas interações que as antecederam. Outro resultado importante é a desigualdade entre as somas de quadrados associadas às hipóteses dos tipos II e III para todos efeitos principais e interaçōes de segundo grau, devida a própria presença de interações no modelo. 
Tabela 24. Análise de variância do delineamento parcialmente ortogonal, descrito na Tabela 20, segundo o modelo $y_{i j k l}=\mu+\alpha_{i}+\beta_{j}+\gamma_{k}+\varphi_{i j}+\eta_{i k}+\tau_{j k}+\rho_{i j k}+e_{i j k l}$, sendo $A$ o primeiro fator declarado entre os efeitos principais e $A{ }^{*} B$ a primeira entre as interaçōes.

\begin{tabular}{|c|c|c|c|c|}
\hline Fontes & $g l$ & S.Q. Tipo I & Q.M. & $R()$. \\
\hline A & 1 & 24,38095238 & 24,38095238 & $R(\alpha \mid \mu)$ \\
\hline B & 1 & 702,17142857 & 702,17242857 & $R(\beta \mid \mu, \alpha)$ \\
\hline c & 1 & 1852,51764706 & 1852,51764706 & $R(\gamma \mid \mu, \alpha, \beta)$ \\
\hline$A * B$ & 1 & 197,48571429 & 197,48571429 & $\boldsymbol{R}(\varphi \mid \mu, \alpha, \beta, \gamma)$ \\
\hline$A * C$ & 1 & 474,91764706 & 474,91764706 & $R(\eta \mid \mu, \alpha, \beta, \gamma, \varphi)$ \\
\hline$B * C$ & 1 & 259,88235294 & 259,88235294 & $R(\tau \mid \mu, \alpha, \beta, \gamma, \varphi, \eta)$ \\
\hline$A * B * C$ & 1 & 497,88235294 & 497,88235294 & $R(\rho \mid \mu, \alpha, \beta, \gamma, \varphi, \eta, \tau)$ \\
\hline Fontes & $g l$ & S.Q. Tipo I & Q.M. & $R()$. \\
\hline A & 1 & 24,38095238 & $2.4,3809.52 .38$ & $\boldsymbol{R}(\alpha \mid \mu, \beta, \gamma)$ \\
\hline B & 1 & 919,11764706 & 919,11764706 & $R(\beta \mid \mu, \alpha, \gamma)$ \\
\hline C & 1 & 1852,51764706 & 1852,51764706 & $R(\gamma \mid \mu, \alpha, \beta)$ \\
\hline$A * B$ & 1 & 144,11764706 & 144,11764706 & $R(\Phi \mid \mu, \alpha, \beta, \gamma, \eta, \tau)$ \\
\hline$A{ }^{*} \mathrm{C}$ & 1 & 474,91764706 & 474,91764706 & $R(\eta \mid \mu, \alpha, \beta, \gamma, \varphi, \tau)$ \\
\hline$B * C$ & 1 & 259,88235294 & 259,88235294 & $R(\tau \mid \mu, \alpha, \beta, \gamma, \varphi, \eta)$ \\
\hline$A \star^{*} \star^{*} C$ & 1 & 497,88235294 & 497,88235294 & $R(\rho \mid \mu, \alpha, \beta, \gamma, \varphi, \eta, \tau)$ \\
\hline Fontes & $g l$ & SQ Tipo $I I I=I V$ & Q.M. & $R()$. \\
\hline A & 1 & 23,52941176 & 23,52941176 & $\boldsymbol{R}(\dot{\alpha} \mid \dot{\mu}, \dot{\beta}, \dot{\gamma})$ \\
\hline$B$ & 1 & 1088,00000000 & 1088,00000000 & $R(\dot{\beta} \mid \dot{\mu}, \dot{\alpha}, \dot{\gamma})$ \\
\hline c & 1 & 737,88235294 & 737,88235294 & $R(\dot{\gamma} \mid \dot{\mu}, \dot{\alpha}, \dot{\beta})$ \\
\hline$A \star B$ & 1 & 113,88235294 & 113,88235294 & $\boldsymbol{R}(\dot{\varphi} \mid \dot{\mu}, \dot{\alpha}, \dot{\beta}, \dot{\gamma}, \dot{\eta}, \dot{\tau})$ \\
\hline$A * C$ & 1 & 113,88235294 & 113,88235294 & $R(\dot{\eta} \mid \dot{\mu}, \dot{\alpha}, \dot{\beta}, \dot{\gamma}, \dot{\varphi}, \dot{\tau})$ \\
\hline$B * C$ & 1 & 60,23529412 & 60,23529412 & $R(\dot{\tau} \mid \dot{\mu}, \dot{\alpha}, \dot{\beta}, \dot{\gamma}, \dot{\varphi}, \dot{\tau})$ \\
\hline$A * B * C$ & 1 & 497,88235294 & 497,88235294 & $R(\dot{p} \mid \dot{\mu}, \dot{\alpha}, \dot{\beta}, \dot{\gamma}, \dot{\varphi}, \dot{\eta}, \dot{\tau})$ \\
\hline
\end{tabular}

A análise da variância da Tabela 25 mostra que tanto para o fator $B$ quanto para a interação $A^{*} C$, a soma de quadrados associada às hipóteses dos tipos I e II são diferentes, ou seja, $R(\beta \mid \mu) \neq R(\beta \mid \mu, \alpha, \gamma)$ e $R(\eta \mid \mu, \alpha, \beta, \gamma) \neq R(\eta \mid \mu, \alpha, \beta, \gamma, \varphi, \tau)$. 
Tabela 25. Análise de variância do delineamento parcialmente ortogonal, descrito na Tabela 20, segundo o modelo $y_{i j k l}=\mu+\beta_{j}+\alpha_{i}+\gamma_{k}+\eta_{i k}+\varphi_{i j}+\tau_{j k}+\rho_{i j k}+e_{i j k l}$, sendo $B$ o primeiro fator declarado entre os efeitos principais e $A{ }^{*} C$ a primeira entre as interações.

\begin{tabular}{|c|c|c|c|c|}
\hline Fontes & $g l$ & S.Q. Tipo I & Q.M. & $R()$. \\
\hline B & 1 & 702,17142857 & $702,17142.857$ & $\boldsymbol{R}(\beta \mid \mu)$ \\
\hline A & 1 & 24,38095238 & 24,38095238 & $R(\alpha \mid \mu, \beta)$ \\
\hline c & 1 & 1852,51764706 & 1852,51764706 & $R(\gamma \mid \mu, \alpha, \beta)$ \\
\hline$A \star C$ & 1 & 528,28571429 & 528,28571429 & $\boldsymbol{R}(\eta \mid \mu, \alpha, \beta, \gamma)$ \\
\hline$A \star B$ & 1 & 144,11764706 & 144,11764706 & $R(\varphi \mid \mu, \alpha, \beta, \gamma, \eta)$ \\
\hline$B{ }^{\star} \mathrm{C}$ & 1 & 259,88235294 & $2.59,88235294$ & $R(\tau \mid \mu, \alpha, \beta, \gamma, \varphi, \eta)$ \\
\hline$A * B{ }^{*} C$ & 1 & 497,88235294 & 497,88235294 & $R(\rho \mid \mu, \alpha, \beta, \gamma, \varphi, \eta, \tau)$ \\
\hline Fontes & $g l$ & S.Q. Tipo II & Q.M. & $R()$. \\
\hline B & 1 & 919,12764706 & 919,11764706 & $\boldsymbol{R}(\beta \mid \mu, \alpha, \gamma)$ \\
\hline A & 1 & 24,38095238 & 24,38095238 & $R(\alpha \mid \mu, \beta, \gamma)$ \\
\hline $\mathrm{C}$ & 1 & 1852,51764706 & 1852,51764706 & $R(\gamma \mid \mu, \alpha, \beta)$ \\
\hline$A \star C$ & 1 & 474,91764706 & 474,91764706 & $R(\eta \mid \mu, \alpha, \beta, \gamma, \Phi, \tau)$ \\
\hline$A * B$ & 1 & 144,11764706 & 144,11764706 & $R(\varphi \mid \mu, \alpha, \beta, \gamma, \eta, \tau)$ \\
\hline$B * C$ & 1 & 259,88235294 & 259,88235294 & $R(\tau \mid \mu, \alpha, \beta, \gamma, \varphi, \eta)$ \\
\hline$A * B * C$ & 1 & 497,88235294 & 497,88235294 & $R(\rho \mid \mu, \alpha, \beta, \gamma, \varphi, \eta, \tau)$ \\
\hline Fontes & $g l$ & SQ Tipo III=IV & Q.M. & $R()$. \\
\hline B & 1 & 1088,00000000 & 1088,00000000 & $\boldsymbol{R}(\dot{\beta} \mid \dot{\mu}, \dot{\alpha}, \dot{\gamma})$ \\
\hline A & 1 & 23,52941176 & 23,52941176 & $R(\dot{\alpha} \mid \dot{\mu}, \dot{\beta}, \dot{\gamma})$ \\
\hline c & 1 & 737,88235294 & 737,88235294 & $R(\dot{\gamma} \mid \dot{\mu}, \dot{\alpha}, \dot{\beta})$ \\
\hline$A * C$ & 1 & 113,88235294 & 113,88235294 & $\boldsymbol{R}(\dot{\eta} \mid \dot{\mu}, \dot{\alpha}, \dot{\beta}, \dot{\gamma}, \dot{\varphi}, \dot{\tau})$ \\
\hline$A \star B$ & 1 & 113,88235294 & 113,88235294 & $R(\dot{\varphi} \mid \dot{\mu}, \dot{\alpha}, \dot{\beta}, \dot{\gamma}, \dot{\eta}, \dot{\tau})$ \\
\hline$B * C$ & 1 & 60,23529412 & 60,23529412 & $R(\dot{\tau} \mid \dot{\mu}, \dot{\alpha}, \dot{\beta}, \dot{\gamma}, \dot{\varphi}, \dot{\tau})$ \\
\hline$A \star B * C$ & 1 & 497,88235294 & 497,88235294 & $R(\dot{\rho} \mid \dot{\mu}, \dot{\alpha}, \dot{\beta}, \dot{\gamma}, \dot{\varphi}, \dot{\eta}, \dot{\tau})$ \\
\hline
\end{tabular}

Assim como ocorreu para os efeitos principais, a desigualdade entre as somas de quadrados associadas às hipóteses dos tipos I e II para algumas interações não implicou na diferença entre todos os projetores ajustados. Um exemplo è a interação $A * B$ onde $R(\varphi \mid \mu, \alpha, \beta, \gamma, \eta)=$ $=R(\varphi \mid \mu, \alpha, \beta, \gamma, \eta, \tau)=144,1176$ apesar da desigualdade entre os tipos I e II. Isto ocorre porque a presença de parâmetros da interação $B^{*} C$ não altera a soma de quadrados da decomposição, pois para $B^{\star} C$ a soma de quadrados associadas 
às hipóteses do tipo I são iguais às do tipo II. Da mesma forma ocorre para a interação $A^{*} C, R(\eta \mid \mu, \alpha, \beta, \gamma)=R(\eta \mid \mu, \alpha, \beta, \gamma, \tau)=474,9176$.

Tabela 26. Análise de variância do delineamento parcialmente ortogonal, descrito na Tabela 20, segundo o modelo $y_{i j k l}=\mu+\gamma_{k}+\beta_{j}+\alpha_{i}+\tau_{j k}+\eta_{i k}+\varphi_{i j}+\rho_{i j k}+e_{i j k l}$, sendo $C$ o primeiro fator declarado entre os efeitos principais e $B^{*} C$ a primeira entre as interaçōes.

\begin{tabular}{|c|c|c|c|c|}
\hline Fontes & $g l$ & S.Q. Tipo I & Q.M. & $R()$. \\
\hline c & 1 & 1635,57142857 & 1635,57142857 & $R(y \mid \mu)$ \\
\hline B & 1 & $919,11 \% 64706$ & $919,11 / 64 / 06$ & $R(\beta \mid \mu, \gamma)$ \\
\hline A & 1 & 24,38095238 & 24,38095238 & $R(\alpha \mid \mu, \beta, \gamma)$ \\
\hline$B * C$ & 1 & 259,88235294 & 259,88235294 & $\boldsymbol{R}(\tau \mid \mu, \alpha, \beta, \gamma)$ \\
\hline$A * B$ & 1 & 528,28571429 & 528,28571429 & $R(\varphi \mid \mu, \alpha, \beta, \gamma, \tau)$ \\
\hline$A^{\star \star C}$ & 1 & 144,11764706 & 144,11764706 & $R(\eta \mid \mu, \alpha, \beta, \gamma, \varphi, \tau)$ \\
\hline$A * B * C$ & 1 & 497,88235294 & 497,88235294 & $R(\rho \mid \mu, \alpha, \beta, \gamma, \varphi, \eta, \tau)$ \\
\hline Fontes & $g l$ & S.Q. Tipo II & Q.M. & $R()$. \\
\hline c & 1 & 1852,51764706 & 1852,51764706 & $R(\gamma \mid \mu, \alpha, \beta)$ \\
\hline B & 1 & 919,11764706 & 919,11764706 & $R(\beta \mid \mu, \alpha, \gamma)$ \\
\hline A & 1 & 24,38095238 & 24,38095238 & $R(\alpha \mid \mu, \beta, \gamma)$ \\
\hline$B * C$ & 1 & 259,88235294 & 259,88235294 & $\boldsymbol{R} \cdot(\tau \mid \mu, \alpha, \beta, \gamma, \varphi, \eta)$ \\
\hline$A \star B$ & 1 & 474,91764706 & 474,91764706 & $R(\varphi \mid \mu, \alpha, \beta, \gamma, \eta, \tau)$ \\
\hline$A \star C$ & 1 & 144,11764706 & 144,11764706 & $R(\eta \mid \mu, \alpha, \beta, \gamma, \varphi, \tau)$ \\
\hline$A{ }^{*} B{ }^{*} C$ & 1 & 497,88235294 & 497,88235294 & $R(\rho \mid \mu, \alpha, \beta, \gamma, \varphi, \eta, \tau)$ \\
\hline Fontes & $g l$ & SQ Tipo III $=I V$ & Q.M. & $R()$. \\
\hline c & 1 & 737,88235294 & 737,89235294 & $\boldsymbol{R}(\dot{\gamma} \mid \dot{\mu}, \dot{\alpha}, \dot{\beta})$ \\
\hline B & 1 & 1088,00000000 & 1088,00000000 & $R(\dot{\beta} \mid \dot{\mu}, \dot{\alpha}, \dot{\gamma})$ \\
\hline A & 1 & 23,52941176 & 23,52941176 & $R(\dot{\alpha} \mid \dot{\mu}, \dot{\beta}, \dot{\gamma})$ \\
\hline$B * C$ & 1 & 60,23529412 & 60,23529412 & $R(\dot{\tau} \mid \dot{\mu}, \dot{\alpha}, \dot{\beta}, \dot{\gamma}, \dot{\varphi}, \dot{\tau})$ \\
\hline$A \star B$ & 1 & 113,88235294 & $: 13,88235294$ & $R(\dot{\varphi} \mid \dot{\mu}, \dot{\alpha}, \dot{\beta}, \dot{\gamma}, \dot{\eta}, \dot{\tau})$ \\
\hline$A * C$ & 1 & 113,88235294 & 113,88235294 & $R(\dot{\eta} \mid \dot{\mu}, \dot{\alpha}, \dot{\beta}, \dot{\gamma}, \dot{\varphi}, \dot{\tau})$ \\
\hline$A \star B * C$ & 1 & 497,88235294 & 497,88235294 & $R(\dot{\rho} \mid \dot{\mu}, \dot{\alpha}, \dot{\beta}, \dot{\gamma}, \dot{\varphi}, \dot{\eta}, \dot{\tau})$ \\
\hline
\end{tabular}

Dentre todas as interações de segunda ordem, a interação formada pelos fatores que não apresentaram freqüências proporcionais entre si, $B^{*} C$ apresentou soma de quadrados associada à hipótese do tipo I igual a do ipo II (Tabela 26). Assim, $R(\tau \mid \mu, \alpha, \beta, \gamma)=R(\tau \mid \mu, \alpha, \beta, \gamma, \varphi, \eta)=259,8824$ que garante também a igualdade com $R(\tau \mid \mu, \alpha, \beta, \gamma, \varphi)$ e $R(\tau \mid \mu, \alpha, \beta, \gamma, \eta)$. 


\subsubsection{Modelos com quatro fatores}

Apresenta-se, a seguir, para os delineamentos parcialmente ortogonais de modelos com quatro fatores, apenas alguns resultados, visando evitar que o texto fique exaustivo em função da notação, do número de tabelas e de quadros.

\subsubsection{6อ Exemplo}

Tabela 27. Dados de porcentagem de germinação de canafístula (Peltophorum dubium Spreng) do delineamento parcialmente ortogonal com quatro fatores.

\begin{tabular}{|c|c|c|c|c|c|c|c|c|}
\hline \multirow[b]{5}{*}{ Temp. (fator $A$ ) } & \multicolumn{8}{|c|}{ Substrato (Fator $B$ ) } \\
\hline & \multicolumn{4}{|c|}{$\begin{array}{c}\dot{j}=1 \\
\text { Dormência (Fator } C \text { ) }\end{array}$} & \multicolumn{4}{|c|}{$\begin{array}{c}\dot{F}^{2} \\
\text { Dormência (Fator } C \text { ) }\end{array}$} \\
\hline & \multicolumn{2}{|c|}{$k=1$} & \multicolumn{2}{|c|}{$k=2$} & \multicolumn{2}{|c|}{$k=1$} & \multicolumn{2}{|c|}{$\vec{k}=2$} \\
\hline & \multicolumn{4}{|c|}{ Épocas (fator $D$ ) } & \multicolumn{4}{|c|}{ Épocas (fator $D$ ) } \\
\hline & $l=1$ & $l=2$ & $l=1$ & $l=2$ & $l=1$ & $l=2$ & $l=1$ & $l=2$ \\
\hline \multirow[t]{6}{*}{$i=1$} & 82 & 72 & 36 & 40 & 62 & 70 & 42 & 48 \\
\hline & 76 & 46 & 30 & 42 & 68 & 62 & & \\
\hline & 70 & 86 & & & 50 & 62 & & \\
\hline & 64 & 64 & & & & & & \\
\hline & 68 & 54 & & & & & & \\
\hline & 76 & 52 & & & & & & \\
\hline \multirow[t]{12}{*}{$i=2$} & 70 & 76 & 38 & 40 & 70 & 70 & 40 & 38 \\
\hline & 90 & 72 & 30 & 48 & 78 & 74 & 40 & 30 \\
\hline & 90 & 74 & 38 & 40 & 72 & 64 & 38 & 32 \\
\hline & 60 & 74 & 28 & 36 & 68 & 68 & & \\
\hline & 86 & 74 & 32 & 42 & 78 & 74 & & \\
\hline & 82 & 56 & 26 & 34 & 84 & 74 & & \\
\hline & 70 & 84 & & & & & & \\
\hline & 76 & 88 & & & & & & \\
\hline & 76 & 82 & & & & & & \\
\hline & 64 & 84. & & & & & & \\
\hline & 70 & 46 & & & & & & \\
\hline & 74 & 70 & & & & & & \\
\hline
\end{tabular}

Para os dados da Tabela 27 foram utilizadas as expressões 21, 22, 23, 24, 25 e 26, considerando o modelo: $y_{i j k l m}=\mu+\alpha_{i}+\beta_{j}+\gamma_{k}+\varphi_{l}+e_{i j k l m}$, onde, para $i=1, \ldots, a$ niveis do fator $A ; j=1, \ldots, b$ niveis do fator $B, k=1, \ldots, c$ niveis do fator $C, l=1, \ldots, d$ niveis do fator $D$ e $m=1, \ldots, n_{i j k}$ observações de cada casela $(i ; j ; k, l)$. Foram obtidos os seguintes esquemas: 
i Ignorando os fatores C e D

\begin{tabular}{|c|c|c|c|c|}
\hline \multirow[b]{2}{*}{ Fator B } & \multicolumn{2}{|c|}{ Fator A } & & \multirow{2}{*}{$\frac{n_{i j . .}}{n_{i j^{\prime} . .}}=\frac{n_{i j^{\prime} . .}}{n_{i j^{\prime} . .}}=2$ ou $\frac{4}{9}$} \\
\hline & $i=1$ & $i=2$ & & \\
\hline$\dot{j}=1$ & $n_{11 . .}=16$ & $n_{21 . .}=36$ & $n_{.1 .}=52$ & $n_{i j . .}=\frac{n_{i \ldots n_{. j . .}}}{n \ldots . . .}, \forall(i, j)$ \\
\hline$j=2$ & $n_{12 . .}=8$ & $n_{22 . .}=18$ & $n_{.2 .}=26$ & $\therefore C\left(X_{\alpha \mid \mu}\right) \perp C\left(X_{\beta \mid}\right.$ \\
\hline$n_{i \ldots}$ & $n_{1 \ldots}=24$ & $n_{2 \ldots}=54$ & $n_{\ldots .}=78$ & \\
\hline
\end{tabular}

ii. Ignorando os fatores B e D

\begin{tabular}{|c|c|c|c|c|}
\hline \multirow[b]{2}{*}{ Fator C } & \multicolumn{2}{|c|}{ Fator A } & \multirow[b]{2}{*}{$n_{k}$} & \multirow{3}{*}{$\begin{array}{l}\frac{n_{i . k .}}{n_{i . k^{\prime} .}} \neq \frac{n_{i \cdot k .}}{n_{i^{\prime} . k^{\prime} .}} \\
n_{i . k .} \neq \frac{n_{i . . .} n_{. k .}}{n \ldots . .}, \forall(i, k)\end{array}$} \\
\hline & $i=1$ & ${ }_{i=2}$ & & \\
\hline$k=1$ & $n_{1.1 .}=18$ & $n_{2.1 .}=36$ & $n_{.1 .}=54$ & \\
\hline$k=2$ & $n_{1.2 .}=6$ & $n_{2.2 .}=18$ & $n_{.2 .}=24$ & $\therefore C\left(X_{\left.\alpha\right|_{u}}\right) \not \subset C\left(X_{\gamma}\right.$ \\
\hline$n_{i \ldots}$ & $n_{1 \ldots}=24$ & $n_{2 \ldots}=54$ & $n \ldots=78$ & \\
\hline
\end{tabular}

iii Ignorando os fatores B e C

\begin{tabular}{|c|c|c|c|c|}
\hline \multirow[b]{2}{*}{ Fator D } & \multicolumn{2}{|c|}{ Fator A } & \multirow[b]{2}{*}{$n_{\ldots l}$} & \multirow{2}{*}{$\frac{n_{i . . l}}{n_{i^{\prime} l}}=\frac{n_{i . l^{\prime}}}{n_{\cdot i^{\prime} l^{\prime}}}=1$ ou $\frac{4}{9}$} \\
\hline & $i=1$ & $i=2$ & & \\
\hline$l=1$ & $n_{1 . .1}=12$ & $n_{2 . .1}=27$ & $n_{\ldots 1}=39$ & $n_{i \ldots} n_{\ldots l}$ \\
\hline$l=2$ & $n_{1.2}=12$ & $n_{2 . .2}=27$ & $n_{\ldots 2}=39$ & $n \ldots .$. \\
\hline$n_{i .}$ & $n_{1 \ldots}=24$ & $n_{2 \ldots}=54$ & $n_{\ldots}=78$ & \\
\hline
\end{tabular}


iv Ignorando os fatores $A$ e D

\begin{tabular}{c|cc|l} 
& \multicolumn{2}{|c|}{ Fator $B$} & \\
Fator $C$ & $j=1$ & \multicolumn{1}{c}{$=2$} & $n_{. k .}$ \\
\hline$k=1$ & $n_{.11 .}=36$ & $n_{.21 .}=18$ & $n_{. .1 .}=54$ \\
$k=2$ & $n_{.12 .}=16$ & $n_{.22 .}=8$ & $n_{.2 .}=24$ \\
\hline$n_{. j . .}$ & $n_{.1 .}=52$ & $n_{.2 .}=26$ & $n_{. . .}=78$
\end{tabular}

$$
\begin{aligned}
& \frac{n_{. j k .}}{n_{. j k .}}=\frac{n_{. j k^{\prime} .}}{n_{. j k^{\prime} .}}=2 \text { ou } \frac{9}{4} \\
& n_{. j k .}=\frac{n_{. j . .} n_{. . k .}}{n . . .}, \forall(j, k) \\
& \therefore C\left(X_{\beta \mid \mu}\right) \perp C\left(X_{\gamma \mid \mu}\right)
\end{aligned}
$$

\section{$v$ Ignorando os fatores A e C}

\begin{tabular}{c|cc|l} 
& \multicolumn{2}{|c|}{ Fator $B$} & \\
Fator $D$ & $j=1$ & $j=2$ & $n_{. .1}$ \\
\hline$l=1$ & $n_{.1 .1}=26$ & $n_{.2 .1}=13$ & $n_{\ldots .1}=39$ \\
$l=2$ & $n_{.1 .2}=26$ & $n_{.2 .2}=13$ & $n_{. .2}=39$ \\
\hline$n_{. j . .}$ & $n_{.1 . .}=52$ & $n_{.2 .}=26$ & $n_{\ldots . .}=78$
\end{tabular}

\begin{tabular}{|c|c|c|c|c|c|}
\hline \multirow[b]{2}{*}{ Fator D } & \multicolumn{2}{|c|}{ Fator C } & \multirow[b]{2}{*}{$n_{\ldots l}$} & \multirow{2}{*}{$\frac{n_{. . k l}}{n_{. . k^{\prime} l}}=$} & \multirow{2}{*}{$\frac{n_{. . k l^{\prime}}}{n_{. . k^{\prime} l^{\prime}}}=1$ ou $\frac{4}{9}$} \\
\hline & $k=1$ & $k=2$ & & & \\
\hline$l=1$ & $n_{.1 .1}=27$ & $n_{.2 .1}=12$ & $n_{\ldots 1}=39$ & & $n, n$ \\
\hline$l=2$ & $n_{.1 .2}=27$ & $n_{.2 .2}=12$ & $n_{\ldots 2}=39$ & $n_{. . k l}=$ & $\frac{\ldots \ldots}{n \ldots}, \forall(k, l)$ \\
\hline$n_{. . k .}$ & $n_{. .1 .}=54$ & $n_{.2 . .}=24$ & $n_{\ldots .}=78$ & & $\left.K_{\gamma \mid \mu}\right) \perp C\left(X_{\phi{ }^{\prime}}\right)$ \\
\hline
\end{tabular}

$$
\begin{aligned}
& \frac{n_{\cdot j \cdot l}}{n_{\cdot j^{\prime} . l}}=\frac{n_{\cdot j \cdot l^{\prime}}}{n_{\cdot j^{\prime} \cdot l^{\prime}}}=1 \text { ou } 2 \\
& n_{\cdot j . l}=\frac{n_{\cdot j . .} n_{. . l}}{n . \ldots}, \forall(j, l) \\
& \therefore C\left(X_{\beta \mid \mu}\right) \perp C\left(X_{\varphi \mid \mu}\right)
\end{aligned}
$$

vi Ignorando os fatores $A$ e B 
Diante das proporcionalidades obtidas, seria. natural concluir inicialmente que o delineamento não é ortogonal, uma vez, que as freqüências de caselas entre os fatopres $A$ e $C$ não são proporcionais. No entanto, os fatores $B$ e $D$ apresentaram freqüências de caselas proporcionais aos demais fatores, caracterizando o delineamento como parcialmente ortogonal, correspondendo a situação 2 e caso 5, apresentada no Quadro 5.

\section{a Modelo sem interaçōes}

Como conseqüência da ortogonalidade parcial as hipóteses do tipo I são iguais as hipóteses do tipo II para os fatores $B$ e $C$ e, as funções estimáveis envolvendo esses fatores apresentam apenas parâmetros relativos aos próprios fatores (Quadro 23).

Quadro 23. Funções estimáveis fornecidas pelo Proc GLM do SAS e respectivas hipóteses sobre os fatores $A, B, C$ e $\mathrm{D}$ para os dados da Tabela 27.

\begin{tabular}{|c|c|c|c|c|c|}
\hline \multicolumn{3}{|c|}{ Tipo I-A } & \multicolumn{3}{|c|}{ Tipo II-A = Tipo III-A = Tipo $I V-A$} \\
\hline & & $\begin{array}{l}\text { Coeficientes } \\
0\end{array}$ & $\begin{array}{l}\text { Ef } \\
T N=\end{array}$ & & $\begin{array}{l}\text { Coeficientes } \\
0\end{array}$ \\
\hline A & $\begin{array}{l}1 \\
2\end{array}$ & $\begin{array}{l}\mathrm{L} 2 \\
-\mathrm{L} 2\end{array}$ & A & $\begin{array}{l}1 \\
2\end{array}$ & $\begin{array}{l}\mathrm{L} 2 \\
-\mathrm{L} 2\end{array}$ \\
\hline B & $\begin{array}{l}1 \\
2\end{array}$ & $\begin{array}{l}0 \\
0\end{array}$ & B & $\begin{array}{l}1 \\
2\end{array}$ & $\begin{array}{l}0 \\
0\end{array}$ \\
\hline C & $\begin{array}{l}1 \\
2\end{array}$ & $\begin{array}{l}0,0833 * \mathrm{~L} 2 \\
-0,0833 * \mathrm{~L} 2\end{array}$ & $c$ & $\frac{1}{2}$ & $\begin{array}{l}0 \\
0\end{array}$ \\
\hline D & i & $\begin{array}{l}0 \\
0\end{array}$ & $\mathrm{D}$ & $\begin{array}{l}1 \\
2\end{array}$ & $\begin{array}{l}0 \\
0\end{array}$ \\
\hline & & ${ }_{2} \gamma_{1}=\alpha_{2}+1 / 12 \gamma_{2}$ & & & $=H_{o}^{4-A}: \alpha_{1}=\alpha_{2}$ \\
\hline
\end{tabular}


continuação

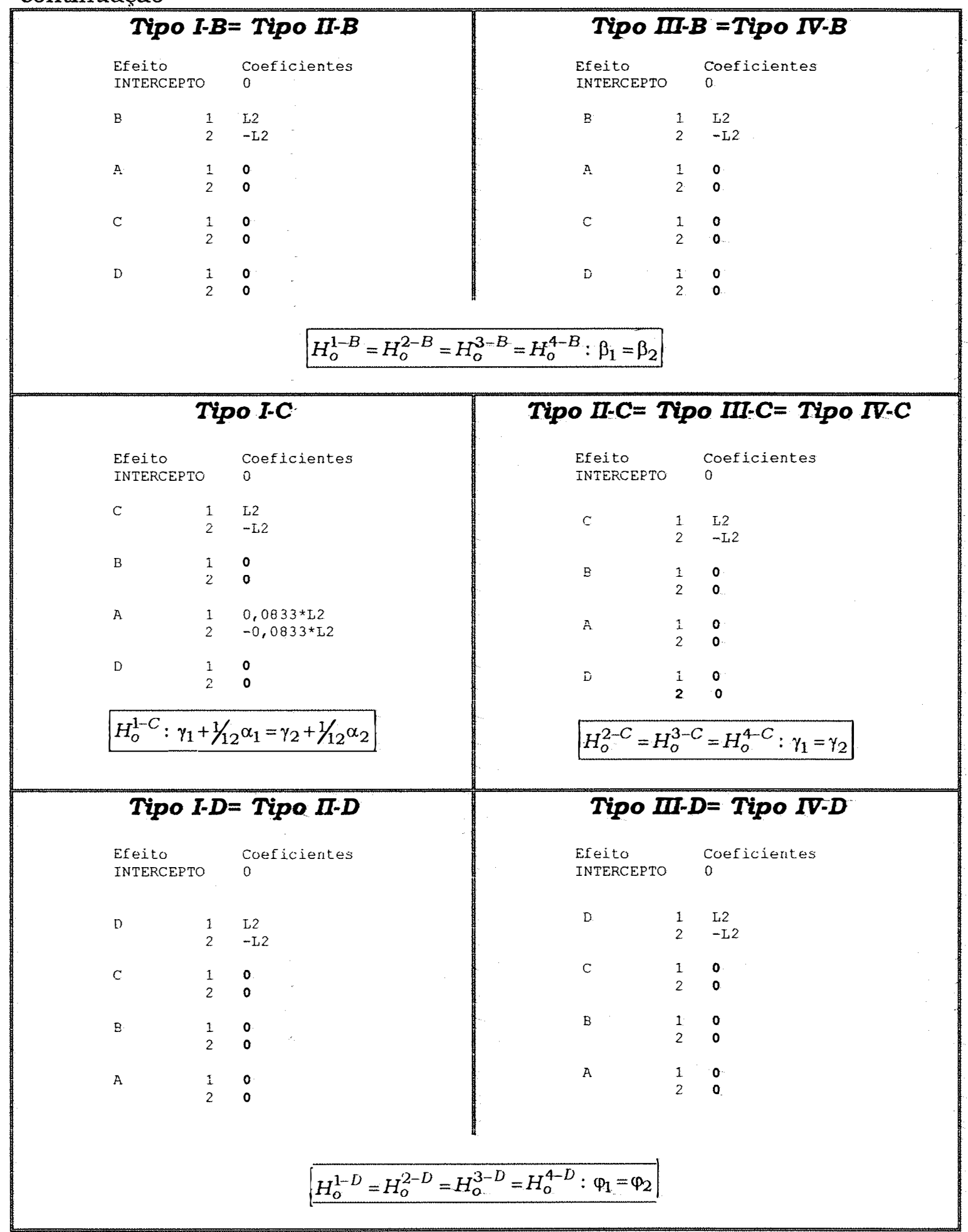


Tabela 28. Análise de variância do delineamento parcialmente ortogonal, descrito na Tabela 27, segundo o modelo $y_{i j k l}=\mu+\alpha_{i}+\beta_{j}+\gamma_{k}+\varphi_{l}+e_{i j k l m}$, declarando $A$ como primeiro fator

\begin{tabular}{|c|c|c|c|c|}
\hline Fontes & $g l$ & S.Q. Tipo I & Q.M. & $R()$. \\
\hline A & 1 & 38,78205128 & 38,78205128 & $R(\alpha \mid \mu)$ \\
\hline B & 1 & 8,30769231 & 8,30769231 & $R(\beta \mid \mu, \alpha)$ \\
\hline C & 1 & 19108,01515152 & 19108,01515152 & $R(\gamma \mid \mu, \alpha, \beta)$ \\
\hline D & 1 & 82,05128205 & 82,05128205 & $R(\varphi \mid \mu, \alpha, \beta, \gamma)$ \\
\hline Fontes & $g l$ & S.Q. Tipe II & Q.M. & $R(:)$ \\
\hline A & 1 & 314,18181818 & 314,18181818 & $R(\alpha \mid \mu, \beta, \gamma, \Phi)$ \\
\hline B & 1 & 8,30769231 & 8,30769231 & $R^{\prime}(\beta+\mu, \alpha, \gamma, \varphi)$ \\
\hline C & 1 & 19108,01515152 & $=9108,01515152$ & $R(\gamma \mid \mu, \alpha, \beta, \varphi)$ \\
\hline D & 1 & 82,05128205 & 82,05128205 & $R(\varphi \mid \mu, \alpha, \beta, \gamma)$ \\
\hline Fontes & $g l$ & $S Q$ Tipo $I I=I V$ & Q.M. & $R()$. \\
\hline A & 1 & 314,18181818 & 314,18181818 & $R(\dot{\alpha} \mid \dot{\mu}, \dot{\beta}, \dot{\gamma}, \varphi)$ \\
\hline B & 1 & 8,30769231 & 8,30769231 & $R(\dot{\beta} \mid \dot{\mu}, \dot{\alpha}, \dot{\gamma}, \varphi)$ \\
\hline C & 1 & 19108,01515152 & 19108,01515152 & $R(\dot{\gamma} \mid \dot{\mu}, \dot{\alpha}, \dot{\beta}, \varphi)$ \\
\hline D & 1 & 82,05128205 & 82,05128205 & $R(\dot{\varphi} \mid \dot{\mu}, \dot{\alpha}, \dot{\beta}, \dot{\gamma})$ \\
\hline
\end{tabular}

Tabela 29. Análise de variância do delineamento parcialmente ortogonal, descrito na Tabela 27, segundo o modelo $y_{i j k l m}=\mu+\beta_{j}+\alpha_{i}+\gamma_{k}+\varphi_{l}+e_{i j k l m}$, declarando $B$ como primeiro-fator.

\begin{tabular}{lrrrl}
\hline Fontes & $g l$ & S.Q.TipoI & Q.M. & $R()$. \\
\hline B & 1 & 8,30769231 & 8,30769231 & $\boldsymbol{R}(\beta \mid \mu)$ \\
A & 1 & 38,18205128 & 38,18205128 & $R(\alpha \mid \mu, \beta)$ \\
C & 1 & 19108,01515152 & 19108,01515152 & $R(\gamma \mid \mu, \alpha, \beta)$ \\
D & 1 & 82,05128205 & 82,05128205 & $R(\varphi \mid \mu, \alpha, \beta, \gamma)$ \\
\hline Fontes & $g l$ & $S . Q . T$ Tipo II=III=IV & $Q . M$. & $R(\cdot)$ \\
\hline B & 1 & 8,30769231 & 8,30769231 & $\boldsymbol{R}(\beta \mid \mu, \alpha, \gamma, \varphi)$ \\
A & 1 & 314,18181818 & 314,18181818 & $R(\alpha \mid \mu, \beta, \gamma, \varphi)$ \\
C & 1 & 19108,01515152 & 19108,01515152 & $R(\gamma \mid \mu, \alpha, \beta, \varphi)$ \\
D & 1 & 82,05128205 & 82,05128205 & $R(\varphi \mid \mu, \alpha, \beta, \gamma)$ \\
\hline
\end{tabular}


Tabela 30. Análise de variância do delineamento parcialmente ortogonal, descrito na Tabela 27, segundo o modelo $y_{i j k l m}=\mu+\gamma_{k}+\alpha_{i}+\beta_{j}+\varphi_{l}+e_{i j k l m}$, declarando $C$ como primeiro fator.

\begin{tabular}{|c|c|c|c|c|}
\hline Fontes & $g l$ & S.Q. Tipo I & Q.M. & $R()$. \\
\hline $\bar{c}$ & 1 & 18832,61538462 & $188.37,6153846 ?$ & $\boldsymbol{R}(\gamma \mid \mu)$ \\
\hline B & 1 & 8,30769231 & 8,30769231 & $R(\beta \mid \mu, \gamma)$ \\
\hline A & 1 & 314,18181818 & 314,18181818 & $R(\alpha \mid \mu, \alpha, \gamma)$ \\
\hline D & 1 & 82,05128205 & 82,05128205 & $R(\varphi \mid \mu, \alpha, \beta, \gamma)$ \\
\hline Fontes & $g l$ & S.Q. Tipo $I I=I I I=I V$ & Q.M. & $R()$. \\
\hline c & 1 & 19108,01515152 & 19108,01515152 & $R(\gamma \mid \mu, \alpha, \beta, \varphi)$ \\
\hline B & 1 & 8,30769231 & 8,30769231 & $R(\beta \mid \mu, \alpha, \gamma, \varphi)$ \\
\hline A & 1 & 314,18181818 & 314,18181818 & $R(\alpha \mid \mu, \beta, \gamma, \varphi)$ \\
\hline D & 1 & 82,05128205 & 82,05128205 & $R(\varphi \mid \mu, \alpha, \beta, \gamma)$ \\
\hline
\end{tabular}

Tabela 31. Análise de variância do delineamento parcialmente ortogonal, descrito. na Tabela 27, segundo o modelo $y_{i j k l m}=\mu+\varphi_{l}+\gamma_{k}+\alpha_{i}+\beta_{j}+e_{i j k l m}$, declarando $D$ como primeiro fator.

\begin{tabular}{lrrrl}
\hline Fontes & $g l$ & S.Q. TipoI & Q.M. & $R(\cdot)$ \\
\hline D & 1 & 82,05128205 & 82,05128205 & $R(\varphi \mid \mu)$ \\
C & 1 & 18832,61538462 & 10832,61538462 & $R(\gamma \mid \mu, \varphi)$ \\
B & 1 & 8,30769231 & 8,30769231 & $R(\beta \mid \mu, \varphi, \gamma)$ \\
A & 1 & 314,18181818 & 314,18181818 & $R(\alpha \mid \mu, \beta, \gamma, \varphi)$ \\
\hline Fontes & $g l$ & S.Q. Tipo II =III=IV & $Q . M$. & $R()$. \\
\hline D & 1 & 82,05128205 & 82,05128205 & $R(\varphi \mid \mu, \alpha, \beta, \gamma)$ \\
C & 1 & 19108,01515152 & 19108,01515152 & $R(\gamma \mid \mu, \alpha, \beta, \varphi)$ \\
B & 1 & 8,30769231 & 8,30769231 & $R(\beta \mid \mu, \alpha, \gamma, \varphi)$ \\
A & 1 & 314,18181818 & 314,18181818 & $R(\alpha \mid \mu, \beta, \gamma, \varphi)$ \\
\hline
\end{tabular}

Na Tabela 28 verifica-se que $R(\alpha \mid \mu) \neq R(\alpha \mid \mu, \beta, \gamma, \varphi)$ e na Tabela 32 que $R(\gamma \mid \mu) \neq R(\gamma \mid \mu, \alpha, \beta, \varphi)$, ou seja, as somas de quadrados associadas às hipóteses dos tipo I e II são diferentes para os fatores $A$ e $C$, respectivamente. No entanto, a igualdade ocorreu para o fator $B, R(\beta \mid \mu)=R\left(\left.\beta\right|_{\mu, \alpha, \gamma, \varphi)=8,3077}\right.$ (Tabela 29) e para o fator $D, R(\varphi \mid \mu)=R(\varphi \mid \mu, \alpha, \beta, \gamma)=82,0513$ (Tabela 31). 


\section{b Modelos com interações}

Como mostrou o Quadro 5, se apenas os fatores $B$ e $D$ apresentam freqüências proporcionais aos demais fatores, então as interações que apresentam igualdade entre as somas de quadrados associadas às hipóteses dos tipos I e II são: $A^{*} C, B^{*} D, A^{*} B^{*} C$ e $A^{*} C{ }^{*} D$ (Tabelas 32 e 33).

Tabela 32. Análise de variância do delineamento parcialmente ortogonal, descrito na Tabela 27 , sendo $B^{*} D$ e $A^{*} B^{*} C$ as primeiras interações declaradas.

\begin{tabular}{|c|c|c|c|}
\hline Fontes & $g l$ & S.Q. Tipo I & Q.M \\
\hline A & 1 & 38,78205128 & 38,78205128 \\
\hline B & 1 & 8,30769231 & 8,30769231 \\
\hline$c$ & 1 & 19108,01515152 & 19108,01515152 \\
\hline D & $i$ & 82,05128205 & 82.05128205 \\
\hline$B * D$ & 1 & 0,41025641 & 0,41025641 \\
\hline$n \star C$ & 1 & 388,04040404 & 388,01040401 \\
\hline$A * B$ & 1 & 10,77564103 & 10,77564103 \\
\hline$A \star D$ & 1 & 0,11538462 & 0,12538462 \\
\hline$B * C$ & 1 & 62,73181818 & 62,73181818 \\
\hline$C \star D$ & 1 & 354,68181818 & 354,68181818 \\
\hline$A * B * C$ & 1 & 112,12626263 & 112,12626263 \\
\hline$n \star B \star D$ & 1 & 210,00641026 & 210,00641026 \\
\hline$A \star C \star D$ & 1 & 17,81818182 & 17,81818182 \\
\hline$B * C * D$ & 1 & 211,28030303 & 211,28030303 \\
\hline$n \star B \star C * D$ & 1 & 1,13636364 & 1,13636364 \\
\hline Fontes & $g l$ & Q.M. Tipo II & Q.M \\
\hline A & 1 & 314,18181818 & 314,18181818 \\
\hline 8 & $i$ & 8,30769231 & 8,30769231 \\
\hline $\mathrm{C}$ & 1 & 19108,01515152 & 19108,01515152 \\
\hline $\mathrm{D}$ & 1 & 82,05128205 & 82,05128205 \\
\hline$B * D$ & 1 & 0,41025641 & 0,41025641 \\
\hline$A * C$ & 1 & 388,04040404 & 388,04040404 \\
\hline$A * B$ & 1 & 6,81818182 & 6,81818182 \\
\hline$A \star D$ & 1 & 1,51515152 & 1,51515152 \\
\hline$B * C$ & 1 & 62,73484848 & 62,73484848 \\
\hline$C * D$ & 1 & 354,68181818 & 354,68181818 \\
\hline$A * B * C$ & 1 & 112,12626263 & 112,12626263 \\
\hline$\Lambda * Q * D$ & 1 & 175,03030303 & 175,03030303 \\
\hline$A * C * D$ & 1 & 17,81818182 & 17,81818182 \\
\hline$B \star C \star D$ & 1 & 211,28030303 & 211,28030303 \\
\hline$A * B * C * D$ & 1 & 1,13635351 & 1,13636361 \\
\hline
\end{tabular}


Tabela 33. Análise de variância do delineamento parcialmente ortogonal, descrito na Tabela 27 , sendo $A^{*} C$ e $A^{*} C{ }^{*} D$ as primeiras interaçōes declaradas.

\begin{tabular}{|c|c|c|c|}
\hline Fontes & $g l$ & S.Q. Tipo I & Q.M. \\
\hline $\bar{A}$ & 1 & 38,78205128 & 38,78205128 \\
\hline B & 1 & 8,30769231 & 8,30769231 \\
\hline C & 2 & 19108,01515152 & 19108,01515152 \\
\hline D & 1 & 82,05128205 & 82,05128205 \\
\hline$A * C$ & 1 & 388,04040404 & 388,04040404 \\
\hline $\mathrm{B} * \mathrm{D}$ & 1 & 0,41025641 & 0.41025541 \\
\hline$A \star B$ & 1 & $10,775.64103$ & 10,77564103 \\
\hline$A * D$ & 1 & 0,11538462 & 0,11538462 \\
\hline$B * C$ & 1 & 62,73484848 & 52,73484848 \\
\hline$C * D$ & 1 & $354 ., 68181818$ & 354,68181818 \\
\hline$A * C * D$ & 1 & 17,81818182 & 17,81818182 \\
\hline$A * B * C$ & 1 & 112,12626263 & 112,12626263 \\
\hline$A * B \star D$ & 1 & 210,00641026 & 210,00641026 \\
\hline$B * C \star D$ & 1 & 211,28030303 & 211,28030303 \\
\hline$A * B * C \star D$ & 1 & 1,13636364 & 1,13636364 \\
\hline Fontes & $g l$ & S.Q.Tipo $B$ & Q.M. \\
\hline$A$ & 1 & 314,18181818 & 314,18181818 \\
\hline B & 1 & 8,30769231 & 8,30769231 \\
\hline$c$ & 1 & 19108,01515152 & 19108,01515152 \\
\hline$D$ & 1 & $82,0512820.5$ & 82,05128205 \\
\hline$A * C$ & 1 & 388,04040404 & 388,04040404 \\
\hline$B * D$ & 1 & 0,11025611 & 0,11025641 \\
\hline$A * B$ & 1 & 6,81818182 & 6,81818182 \\
\hline$A * D$ & 1 & 1,51515152 & 1,51515152 \\
\hline $\mathrm{B}^{*} \mathrm{C}$ & 1 & 62,73484848 & 52,73484848 \\
\hline$C * D$ & 1 & 354,68181818 & 354,68181818 \\
\hline$A * C * D$ & 1 & 17,81818182 & 17,81818182 \\
\hline$A \star B \star C$ & 1 & 112,12626263 & 112,12626263 \\
\hline$A \star B \star D$ & 1 & 175,03030303 & 175,03030303 \\
\hline$B \star C \star D$ & 1 & 211,28030303 & 211,28030303 \\
\hline$A * B \star C * D$ & 1 & 1,13636364 & 1,13636364 \\
\hline
\end{tabular}




\subsection{Planejamento de delineamentos ortogonais e parcialmente ortogonais}

Neste item estão ilustradas a partir de diagramas que representam a ortogonalidade entre subespaços gerados pelas submatrizes de $X$ referentes a cada fator, a igualdade entre hipóteses de delineamentos ortogonais, balanceados ou desbalanceados e, parcialmente ortogonais. Com os diagramas, o pesquisador obtém uma orientação para determinar qual ou quais combinações de fatores deverão apresentar freqüências proporcionais para que as hipóteses de seu interesse, tanto para efeitos principais quanto para interações, sejam testáveis.

É importante ressaltar que delineamentos ortogonais ou parcialmente ortogonais de conjuntos desbalanceados deverão ser planejados, pois não é possivel esperar que um desbalanceamento acidental gere freqüências de caselas proporcionais entre fatores. Nesses casos, se o grau de desbalanceamento permitir, pode ser conveniente que algumas observações sejam retiradas aleatoriamente do conjunto de dados, de modo a obter ortogonalidade. Nesse contexto, as hipóteses de interesse passam a ser testáveis, como inicialmente planejado.

\subsubsection{Planejamento de delineamentos ortogonais}

\subsubsection{Modelos com dois fatores}

Quadro 24. Esquema para o planejamento de delineamentos ortogonais de modelos com dois fatores, com algumas das hipóteses testáveis.

\section{Delineamentos ortogonais com dois fatores (1)}

$$
A-B
$$

\section{Efeitos principais}

$H_{o}^{1-A}=H_{o}^{2-A} ; \quad H_{o}^{1-B}=H_{o}^{2-B}$

\section{Interação}

$H_{o}^{1-A B}=H_{o}^{2-A B}=H_{o}^{3-A B}=H_{o}^{4-A B}$

(1) O traço *___ nos diagramas, entre as letras maiúsculas que descrevem os fatores, indica que o subespaço gerado pelas colunas da submatriz de $X$ referentes a um fator é ortogonal ao subespaço gerado pelas colunas da submatriz de $X$ referentes ao outro fator. 
a Exemplos de freqüências de caselas

\section{a. 1 Conjunto balanceado}

\begin{tabular}{c|cc|} 
& \multicolumn{2}{|c|}{ Fator $B$} \\
Fator $A$ & $j=1$ & $\mathrm{j}=2$ \\
\hline$i=1$ & 3 & 3 \\
$i=2$ & 3 & 3 \\
\hline
\end{tabular}

\section{a.2 Conjunto desbalanceado}

\begin{tabular}{c|cc|} 
& \multicolumn{2}{|c|}{ Fator $B$} \\
Fator $A$ & $j=1$ & $\dot{j} 2$ \\
\hline$i=1$ & 9 & 3 \\
$i=2$ & 6 & 2 \\
\hline
\end{tabular}

\subsubsection{Modelos com três fatores}

Quadro 25. Esquema para o planejamento de delineamentos ortogonais de modelos com três fatores, com algumas das hipóteses testáveis.

Delineamentos ortogonais com três fatores (1)

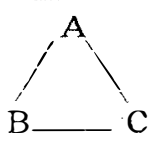

Efeitos principais

$H_{o}^{1-A}=H_{o}^{2-A} ; H_{o}^{1-B}=H_{o}^{2-B}$

$$
H_{o}^{1-C}=H_{o}^{2-C}
$$

$$
\begin{gathered}
\text { Interaçōes duplas } \\
H_{o}^{1-A B}=H_{o}^{2-A B} ; H_{o}^{1-A C}=H_{o}^{2-A C} ; \\
H_{o}^{1-B C}=H_{o}^{2-B C}
\end{gathered}
$$

\section{Interação tripla}

$$
H_{o}^{1-A B C}=H_{o}^{2-A B C}=H_{o}^{3-A B C}=H_{o}^{4-A B C}
$$

(1) O traço * " nos diagramas, entre as letras maiúsculas que descrevem os fatores, indica que o subespaço gerado pelas colunas da submatriz de $X$ referentes a um fator é ortogonal ao subespaço gerado pelas colunas da submatriz de $X$ referentes ao outro fator.

\section{a Exemplos de freqüências de caselas}

\section{a.1 Conjunto balanceado}

\begin{tabular}{c|c|cc|}
\multicolumn{2}{c|}{ Fatores } & \multicolumn{2}{c|}{ Fator C } \\
$A$ & $B$ & $k=1$ & $k=2$ \\
\hline$i=1$ & $j=1$ & 5 & 5 \\
& $j=2$ & 5 & 5 \\
\hline$i=2$ & $j=1$ & 5 & 5 \\
& $j=2$ & 5 & 5 \\
\hline
\end{tabular}

\section{a.2 Conjunto desbalanceado}

Fatores Fator $C$

\begin{tabular}{c|c|cc|}
$A$ & $B$ & $k=1$ & $k=2$ \\
\hline$i=1$ & $j=1$ & 4 & 2 \\
& $j=2$ & 2 & 1 \\
\hline$i=2$ & $j=1$ & 8 & 4 \\
& $j=2$ & 4 & 2 \\
\hline
\end{tabular}




\subsubsection{Modelos com quatro fatores}

Quadro 26. Esquema para o planejamento de delineamentos ortogonais de modelos com quatro fatores, com algumas das hipóteses testáveis.

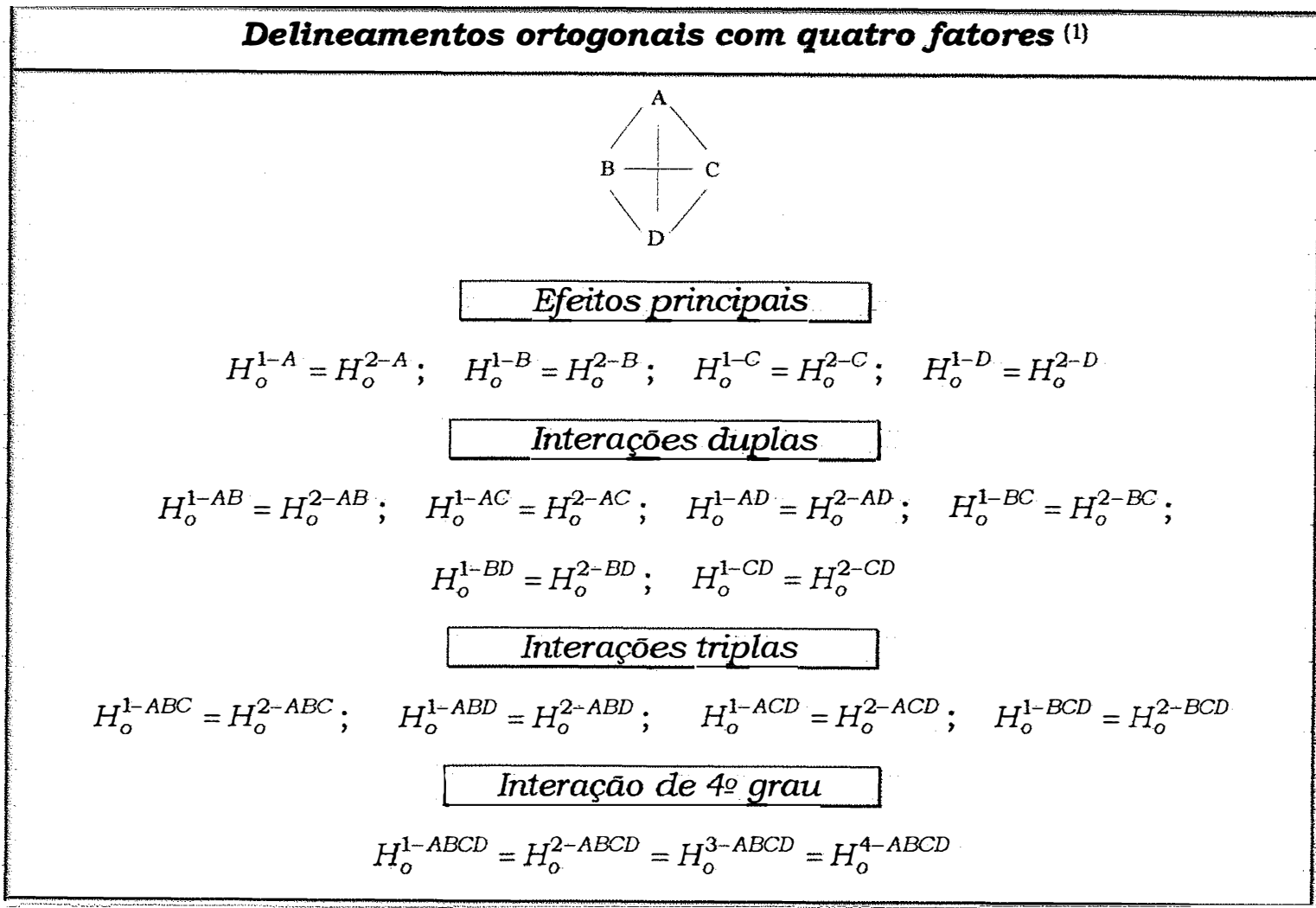

(1) O traço "_ nos diagramas, entre as letras maiúsculas que descrevem os fatores, indica que o subespaço gerado pelas colunas da submatriz de $X$ referentes a um fator é ortogonal ao subespaço gerado pelas colunas da submariz de $X$ referentes ao outro fator.

a Exemplos de freqüências de caselas

\section{a. 1 Conjunto balanceado}

\begin{tabular}{c|c|c|cc|}
\multicolumn{3}{|c|}{ Fatores } & \multicolumn{2}{c|}{ Fator D } \\
$A$ & $B$ & $C$ & $l=1$ & $l=2$ \\
\hline \multirow{2}{*}{$=1$} & $\dot{j} 1$ & $k=1$ & 2 & 2 \\
& & $k=2$ & 2 & 2 \\
\cline { 2 - 5 } & $\dot{j}=2$ & $k=1$ & 2 & 2 \\
& & $k=2$ & 2 & 2 \\
\hline \multirow{3}{*}{$i=2$} & $\dot{j}=1$ & $k=1$ & 2 & 2 \\
& & $k=2$ & 2 & 2 \\
\cline { 2 - 5 } & $\dot{j}=2$ & $k=1$ & 2 & 2 \\
& & $k=2$ & 2 & 2 \\
\hline
\end{tabular}

a.2 Conjunto desbalanceado

\begin{tabular}{c|c|c|cc|}
\multicolumn{3}{c|}{ Fatores } & \multicolumn{2}{c|}{ Fator D } \\
$A$ & $B$ & $C$ & $k=1$ & $l=2$ \\
\hline \multirow{2}{*}{$i=1$} & $j=1$ & $k=1$ & 2 & 4 \\
& & $k=2$ & 1 & 2 \\
\cline { 2 - 5 } & $j=2$ & $k=1$ & 6 & 12 \\
& & $k=2$ & 3 & 6 \\
\hline \multirow{2}{*}{$i=2$} & $j=1$ & $k=1$ & 4 & 8 \\
& & $k=2$ & 2 & 4 \\
\cline { 2 - 5 } & $j=2$ & $k=1$ & 12 & 24 \\
& & $k=2$ & 6 & 12 \\
\hline
\end{tabular}




\subsubsection{Modelos com cinco fatores}

Quadro 27. Esquema para o planejamento de delineamentos ortogonais de modelos com cinco fatores, com algumas das hipóteses testáveis.

\section{Delineamentos ortogonais com cinco fatores (1)}

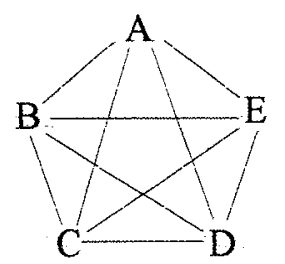

\section{Efeitos principais}

$H_{o}^{1-A}=H_{o}^{2-A} ; \quad H_{o}^{1-B}=H_{o}^{2-B} ; \quad H_{o}^{1-C}=H_{o}^{2-C} ; \quad H_{o}^{1-D}=H_{o}^{2-D} ; H_{o}^{1-E}=H_{o}^{2-E}$

\section{Interaçōes duplas}

$H_{o}^{1-A B}=H_{o}^{2-A B} ; \quad H_{o}^{1-A C}=H_{o}^{2-A C} ; \quad H_{o}^{1-A D}=H_{o}^{2-A D} ; H_{o}^{1-A E}=H_{o}^{2-A E} ; \quad H_{o}^{1-B C}=H_{o}^{2-B C} ;$

$H_{o}^{1-B D}=H_{o}^{2-B D} ; H_{o}^{1-B E}=H_{o}^{2-B E} ; H_{o}^{1-C D}=H_{o}^{2-C D} ; H_{o}^{1-C E}=H_{o}^{2-C E} ; H_{o}^{1-D E}=H_{o}^{2-D E}$

\section{Interaçōes triplas}

$H_{o}^{1-A B C}=H_{o}^{2-A B C} ; \quad H_{o}^{1-A B D}=H_{o}^{2-A B D} ; H_{o}^{1-A B E}=H_{o}^{2-A B E} ; H_{o}^{1-A C D}=H_{o}^{2-A C D} ;$

$H_{o}^{1-A C E}=H_{o}^{2-A C E} ; H_{o}^{1-A D E}=H_{o}^{2-A D E} ; H_{o}^{1-B C D}=H_{o}^{2-B C D} ; H_{o}^{1-B C E}=H_{o}^{2-B C E} ;$

$H_{o}^{1-B D E}=H_{o}^{2-B D E} ; H_{o}^{1-C D E}=H_{o}^{2-C D E}$

\section{Interaçōes de 4o grau}

$H_{o}^{1-A B C D}=H_{o}^{2-A B C D} ; H_{o}^{1-A B C E}=H_{o}^{2-A B C E} ; H_{o}^{1-A B D E}=H_{o}^{2-A B D E} ; H_{o}^{1-A C D E}=H_{o}^{2-A C D E} ;$

$$
H_{0}^{1-B C D E}=H_{0}^{2-B C D E}
$$

$$
\begin{gathered}
\text { Interação de 5o grau } \\
H_{o}^{1-A B C D E}=H_{o}^{2-A B C D E}=H_{o}^{3-A B C D E}=H_{o}^{4-A B C D E}
\end{gathered}
$$

(1) O traço *__ nos diagramas, entre as letras maiúsculas que descrevem os fatores, indica que o subespaço gerado pelas colunas da submatriz de $X$ referentes a um fator é ortogonal ao subespaço gerado pelas colunas da submatriz de $X$ referentes ao outro fator. 


\section{Exemplos de freqüências de caselas}

\section{a.1 Conjunto balanceado}

\begin{tabular}{|c|c|c|c|c|c|}
\hline \multicolumn{4}{|c|}{ Fatores } & \multicolumn{2}{|c|}{ Fator $E$} \\
\hline A & $B$ & $C$ & $D$ & $m=1$ & $m=2$ \\
\hline \multirow[t]{8}{*}{$i=1$} & \multirow[t]{4}{*}{$J=1$} & \multirow[t]{2}{*}{$k=1$} & $l=1$ & 4 & 4 \\
\hline & & & $l=2$ & 4 & 4 \\
\hline & & \multirow[t]{2}{*}{$k=2$} & $l=1$ & 4 & 4 \\
\hline & & & $l=2$ & 4 & 4 \\
\hline & \multirow{4}{*}{$\dot{j}=2$} & \multirow[t]{2}{*}{$k=1$} & $l=1$ & 4 & 4 \\
\hline & & & $l=2$ & 4 & 4 \\
\hline & & \multirow[t]{2}{*}{$k=2$} & $l=1$ & 4 & 4 \\
\hline & & & $l=2$ & 4 & 4 \\
\hline \multirow[t]{8}{*}{$i=2$} & \multirow{4}{*}{$\dot{j}=1$} & \multirow[t]{2}{*}{$k=1$} & $t=1$ & 4 & 4 \\
\hline & & & $l=2$ & 4 & 4 \\
\hline & & \multirow[t]{2}{*}{$k=2$} & $l=1$ & 4 & 4 \\
\hline & & & $l=2$ & 4 & 4 \\
\hline & \multirow{2}{*}{$\dot{j}=2$} & \multirow[t]{2}{*}{$k=1$} & $l=1$ & 4 & 4 \\
\hline & & & $l=2$ & 4 & 4 \\
\hline & & \multirow[t]{2}{*}{$k=2$} & $l=1$ & 4 & 4 \\
\hline & & & $l=2$ & 4 & 4 \\
\hline
\end{tabular}

\section{a.2 Conjunto desbalanceado}

\begin{tabular}{|c|c|c|c|c|c|}
\hline \multicolumn{4}{|c|}{ Fatores } & \multicolumn{2}{|c|}{ Fator $E$} \\
\hline A & $B$ & C & $D$ & $m=1$ & $m=2$ \\
\hline \multirow[t]{8}{*}{$i=1$} & \multirow[t]{4}{*}{$\dot{F} 1$} & $k=1$ & $l=1$ & 2 & 4 \\
\hline & & & $l=2$ & 1 & 2 \\
\hline & & $k=2$ & $l=1$ & 6 & 12 \\
\hline & & & $l=2$ & 3 & 6 \\
\hline & \multirow[t]{4}{*}{$\dot{j}=2$} & $k=1$ & $l=1$ & 4 & 8 \\
\hline & & & $l=2$ & 2 & 4 \\
\hline & & $k=2$ & $l=1$ & 12 & 24 \\
\hline & & & $l=2$ & 6 & 12 \\
\hline \multirow[t]{8}{*}{$i=2$} & \multirow[t]{4}{*}{$\dot{F}=1$} & $k=1$ & $l=1$ & 4 & 8 \\
\hline & & & $l=2$ & - 2 & 4 \\
\hline & & $k=2$ & $l=1$ & 12 & 24 \\
\hline & & & $l=2$ & 6 & 12 \\
\hline & \multirow[t]{4}{*}{$\dot{j}=2$} & $k=1$ & $E=1$ & 8 & 16 \\
\hline & & & $l=2$ & 4 & 8 \\
\hline & & $k=2$ & $l=1$ & 24 & 48 \\
\hline & & & $l=2$ & 12 & 24 \\
\hline
\end{tabular}

\subsubsection{Planejamento de delineamentos parcialmente ortogonais}

Um destaque no estudo dos conjuntos desbalanceados consiste nos delineamentos parcialmente ortogonais que, como citado anteriormente, permitem que a proporcionalidade entre as freqüências de caselas ocorra apenas para os fatores de maior interesse. Essa caracteristica acarreta no. planejamento situações distintas nos testes das hipóteses para os efeitos principais. e interações. Seguindo as propriedades apresentadas no item. 3.2.3.4 do material e métodos, os Quadros 28, 29, 30, 31, 32 e 33, ilustram de maneira resumida, as conseqüências da ortogonalidade parcial em modelos com até cinco fatores. Contudo, nos modelos com cinco fatores os exemplos de freqüências de caselas não foram apresentados pela própria dificuldade de interpretação dos resultados do experimento oriundos desses modelos. 


\subsubsection{Modelos com três fatores}

Quadro 28. Esquema para o planejamento de delineamentos parcialmente ortogonais de modelos com rês fatores, com algumas das hipóteses testáveis.

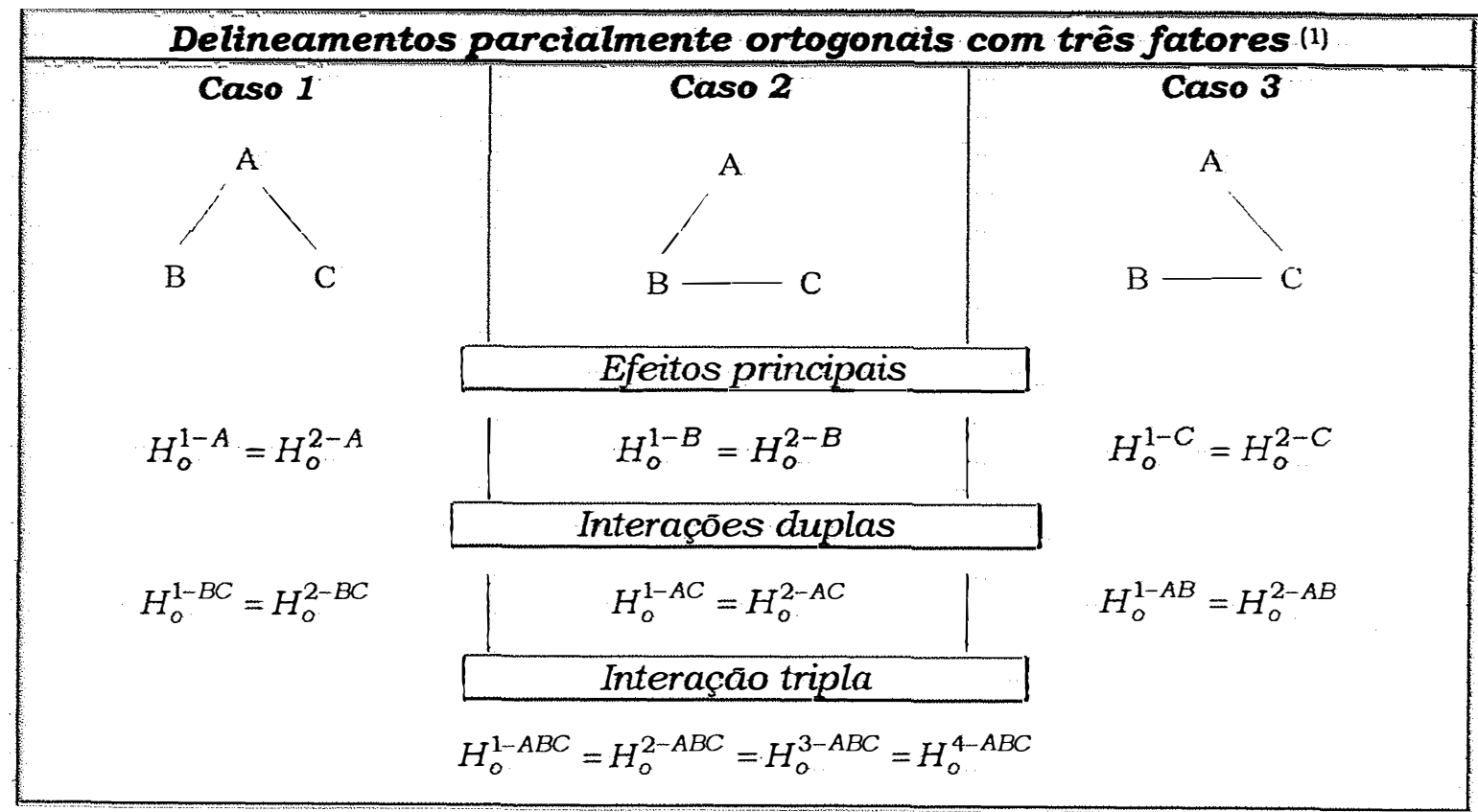

(1) O traço "__ nos diagramas, entre as letras maiúsculas que descrevem os fatores, indica que o subespaço gerado pelas colunas da submatriz de $X$ referentes a um fator é ortogonal ao subespaço gerado pelas colunas da submatriz de $X$ referentes ao outro fator.

a Exemplos de freqüências de caselas

a.1 Caso 1

\section{Fatores}

\begin{tabular}{c|c|cc|}
$A$ & $B$ & $k=1$ & $k=2$ \\
\hline$i=1$ & $j=1$ & 1 & 2 \\
& $j=2$ & 4 & 12 \\
\hline$i=2$ & $j=1$ & 2 & 4 \\
& $j=2$ & 8 & 24 \\
\hline
\end{tabular}

a. 2 Caso 2

Fatores

Fator C

\begin{tabular}{c|c|cc|}
$A$ & $B$ & $k=1$ & $k=2$ \\
\hline$i=1$ & $j=1$ & 10 & 6 \\
& $j=2$ & 5 & 3 \\
\hline$i=2$ & $j=1$ & 14 & 4 \\
& $j=2$ & 7 & 2 \\
\hline
\end{tabular}

\section{a.3 Caso 3}

\begin{tabular}{c|c|cc|}
\multicolumn{2}{c|}{ Fatores } & \multicolumn{2}{c|}{ Fator $C$} \\
$A$ & $B$ & $k=1$ & $k=2$ \\
\hline$i=1$ & $j=1$ & 1 & 2 \\
& $j=2$ & 3 & 6 \\
\hline$i=2$ & $j=1$ & 2 & 4 \\
& $j=2$ & 4 & 8 \\
\hline
\end{tabular}




\subsubsection{Modelos com quatro fatores}

Quadro 29. Esquema para o planejamento de delineamentos parcialmente ortogonais de modelos com quatro fatores, com algumas das hipóteses testáveis para os casos onde um dos fatores é individualmente ortogonal aos demais (situação 1).

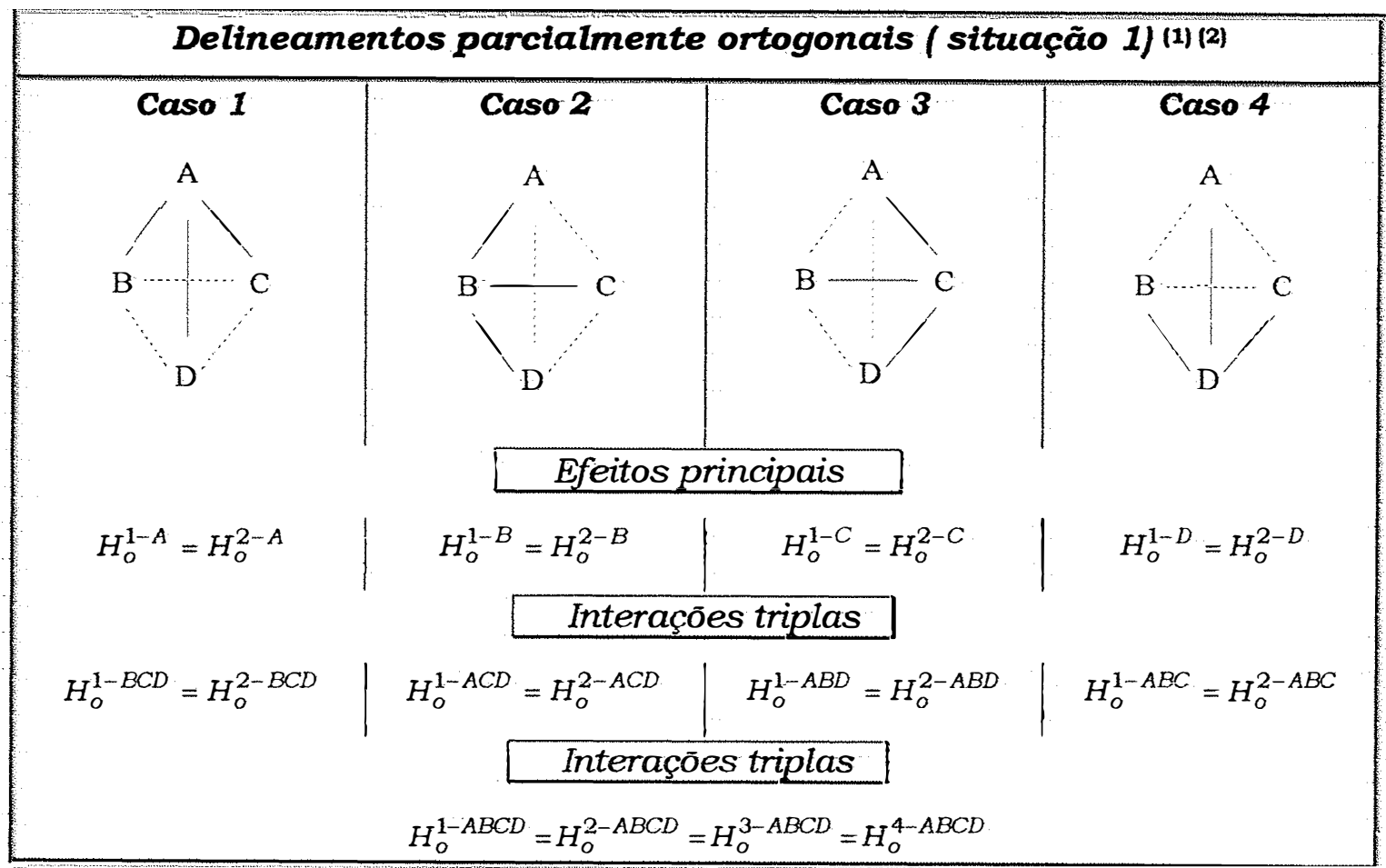

(1) O traço * _ nos diagramas, entre as letras maiúsculas que descrevem os fatores, indica que o subespaço gerado pelas colunas da submatriz de $X$ referentes a um fator é ortogonal ao subespaço gerado pelas colunas da submatriz de $X$ referentes ao outro fator;

(2) O pontilhado " ....... indica que se uma das ortogonalidades ocorrer, as igualdades e as hipóteses testáveis não se alteram.

\section{a. Exemplos de freqüências de caselas}

\section{a. 1 Situação 1 caso 1}

\begin{tabular}{|c|c|c|c|c|}
\hline \multicolumn{3}{|c|}{ Fatores } & \multicolumn{2}{|c|}{ Fator D } \\
\hline$A$ & $B$ & $C$ & $l=1$ & $l=2$ \\
\hline \multirow[t]{4}{*}{$i=1$} & \multirow{2}{*}{$\dot{j}=1$} & $k=1$ & 2 & 1 \\
\hline & & $k=2$ & 4 & 2 \\
\hline & \multirow[t]{2}{*}{$\dot{j}=2$} & $k=1$ & 6 & 2 \\
\hline & & $k=2$ & 4 & 2 \\
\hline \multirow[t]{4}{*}{$i=2$} & \multirow[t]{2}{*}{$\dot{j}=1$} & $k=1$ & 2 & 1 \\
\hline & & $k=2$ & 4 & 2 \\
\hline & \multirow[t]{2}{*}{$j=2$} & $k=1$ & 6 & 2 \\
\hline & & $k=2$ & 4 & 2 \\
\hline
\end{tabular}

a.2 Situação 2 caso 5

\begin{tabular}{c|c|c|cc|}
\multicolumn{3}{c|}{ Fatores } & \multicolumn{2}{c|}{ Fator D } \\
$A$ & $B$ & $C$ & $k=1$ & $l=2$ \\
\hline$i=1$ & $j=1$ & $k=1$ & 1 & 2 \\
& & $k=2$ & 2 & 4 \\
\cline { 2 - 5 } & $\dot{j} 2$ & $k=1$ & 1 & 2 \\
& & $k=2$ & 2 & 4 \\
\hline \multirow{2}{*}{$i=2$} & $\dot{j}=1$ & $k=1$ & 1 & 2 \\
& & $k=2$ & 3 & 6 \\
\cline { 2 - 5 } & $\dot{j}=2$ & $k=1$ & 1 & 2 \\
& & $k=2$ & 3 & 6 \\
\hline
\end{tabular}




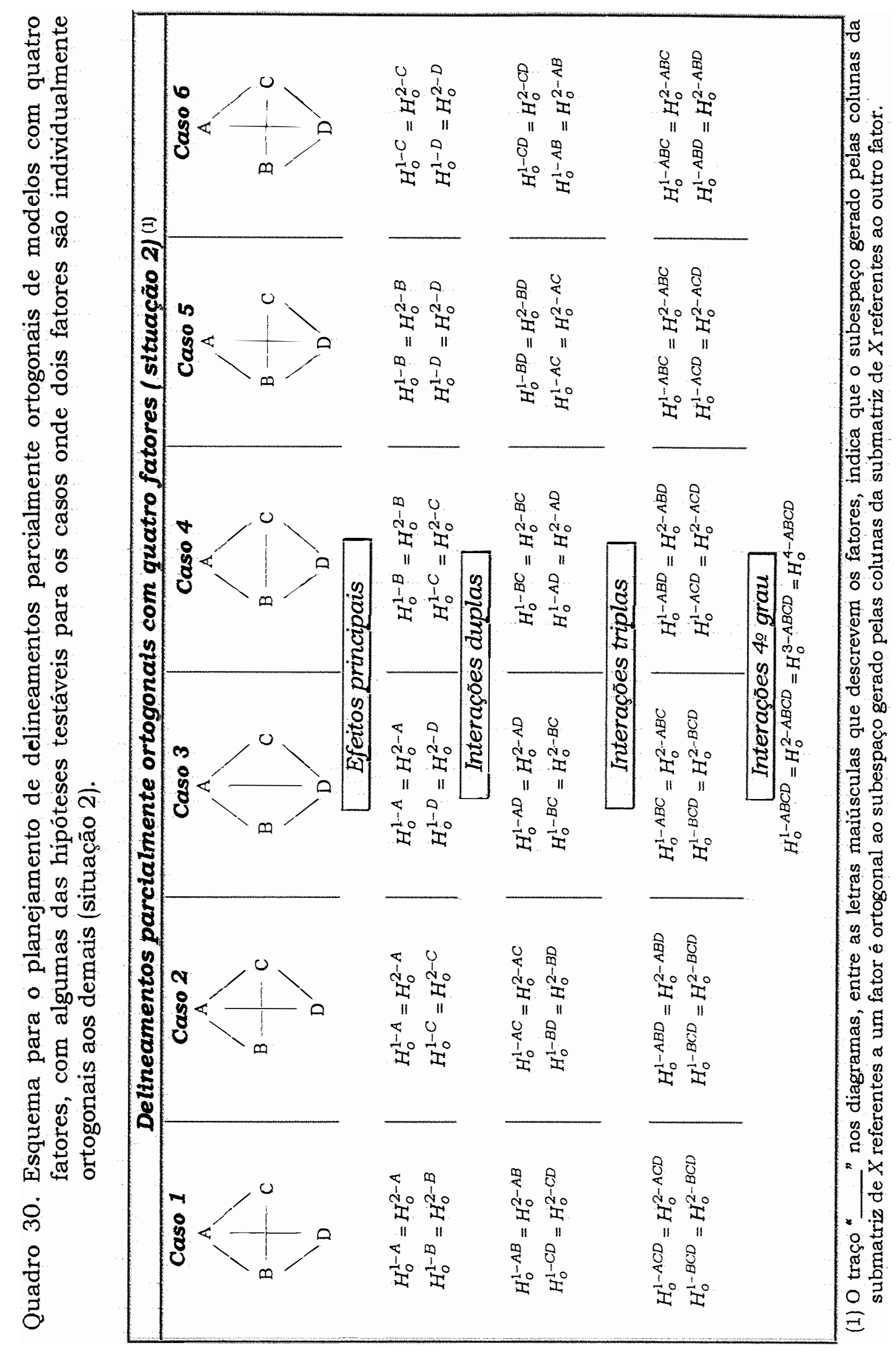

121 


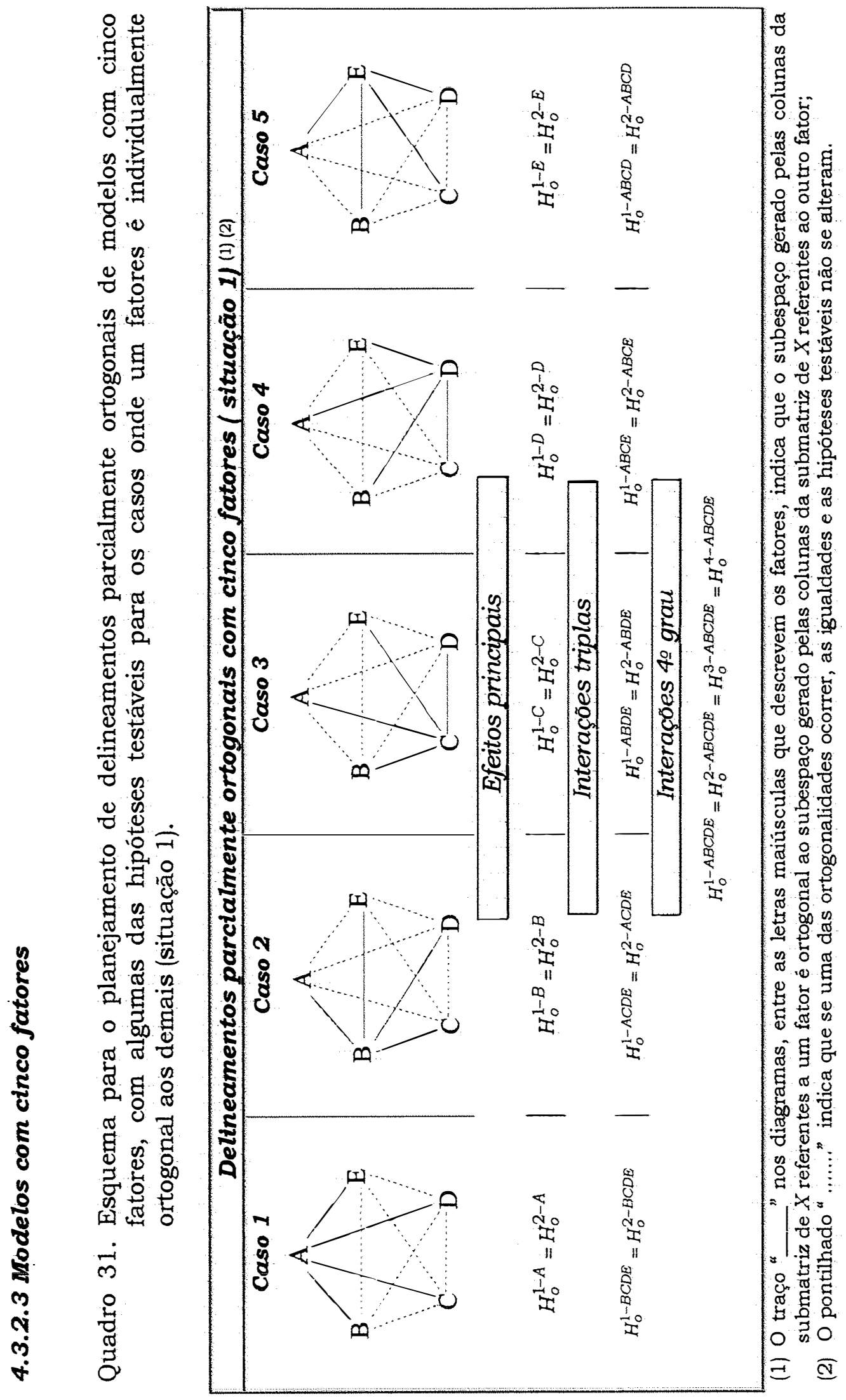




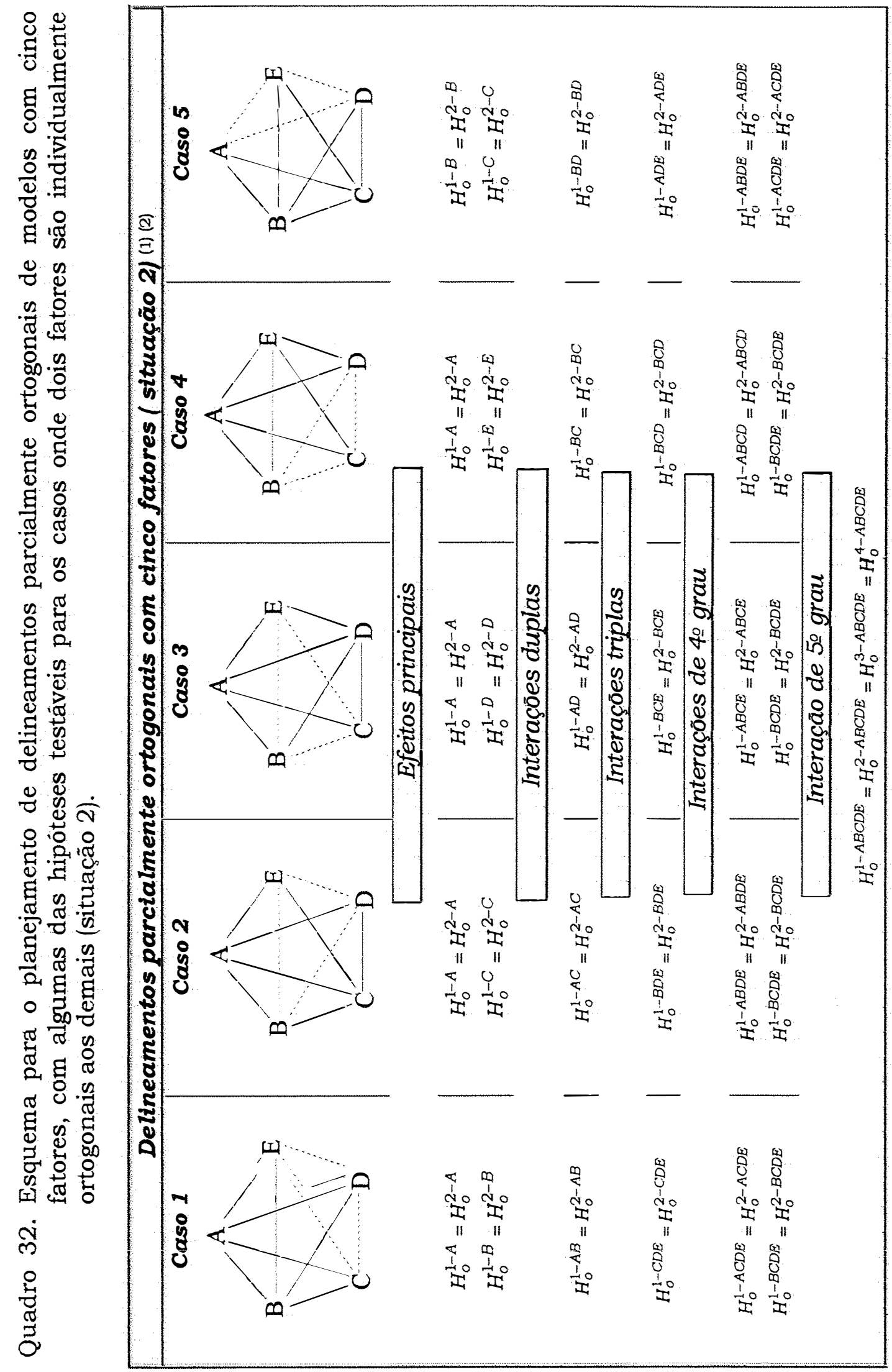




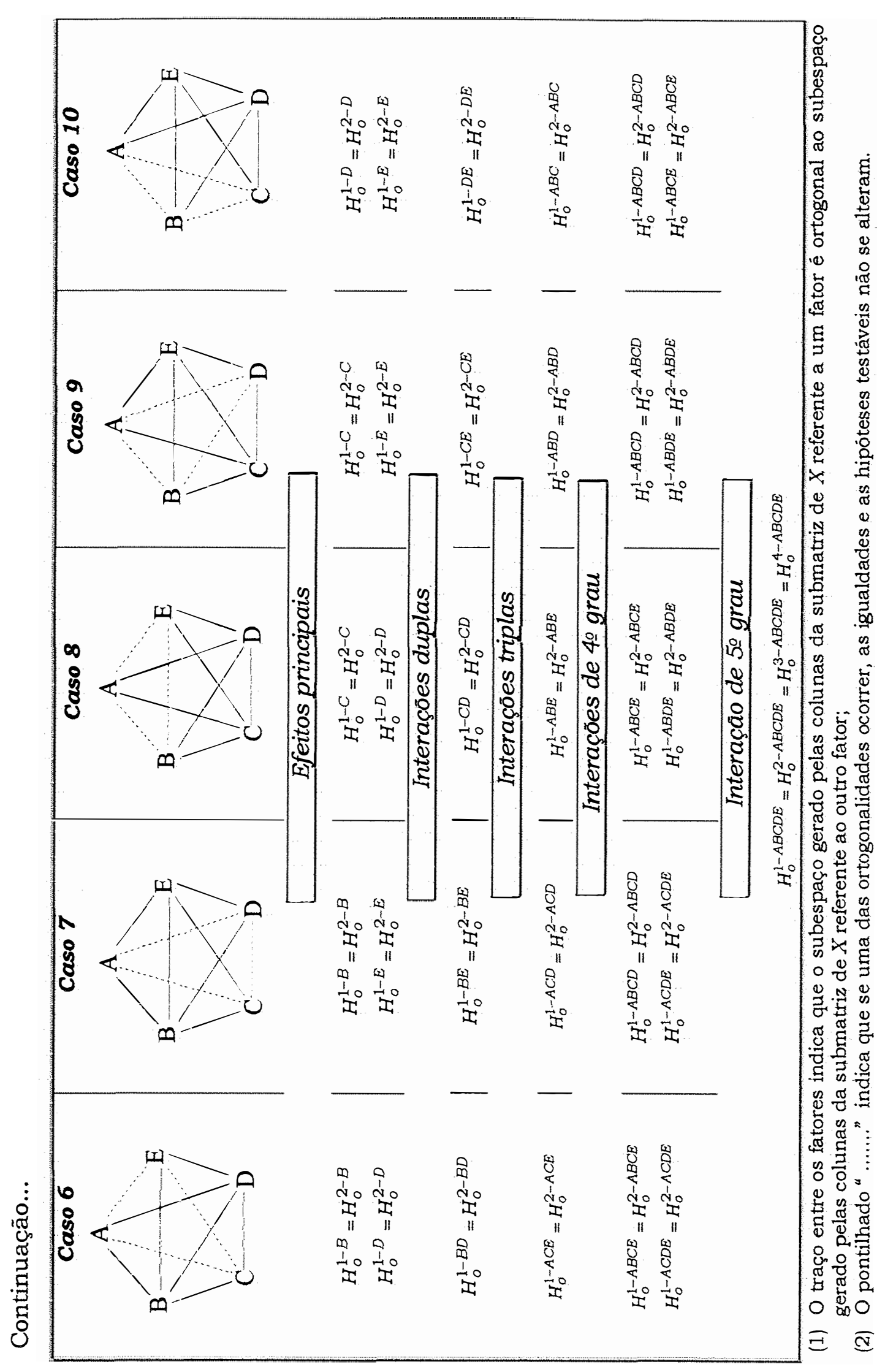




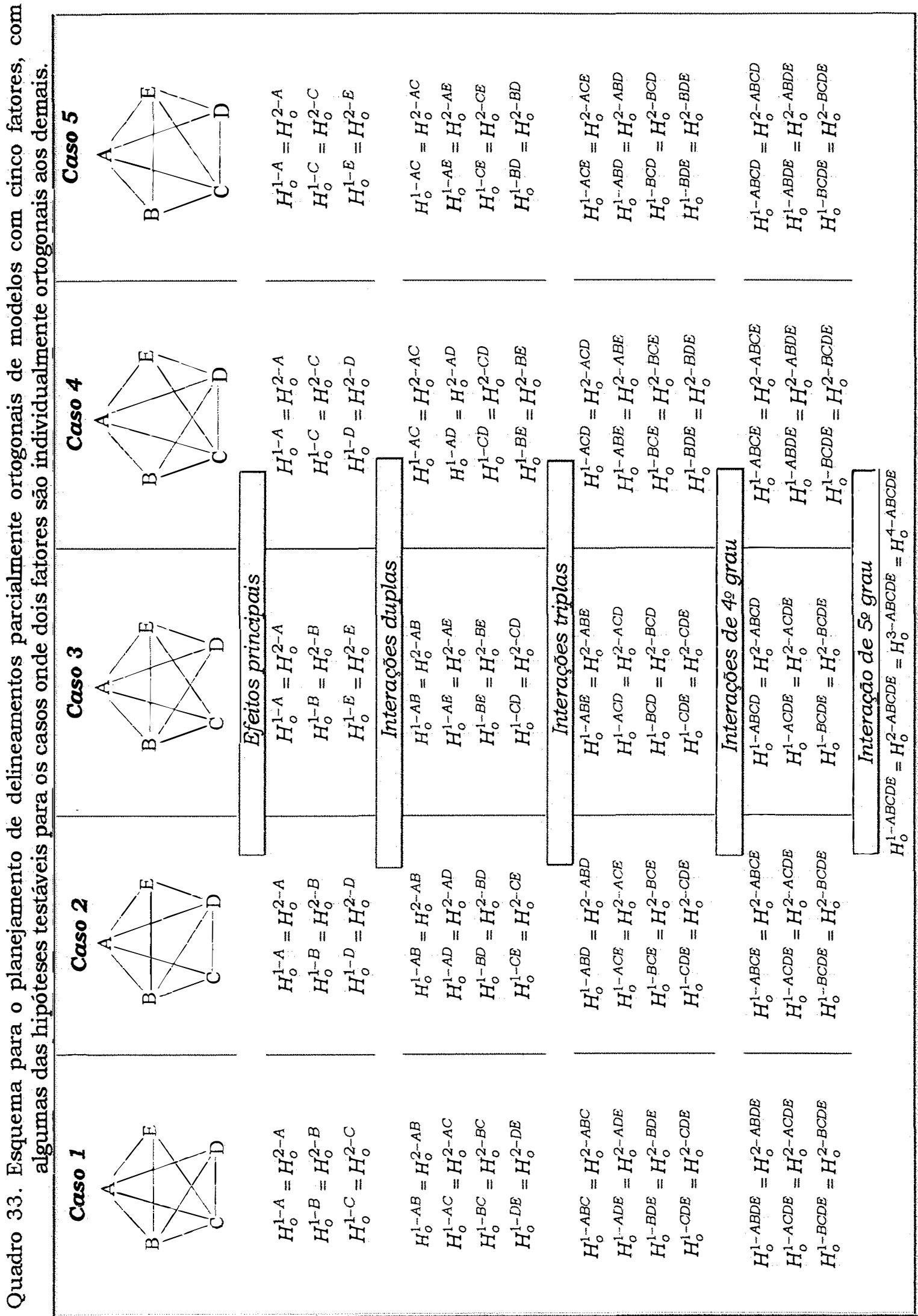




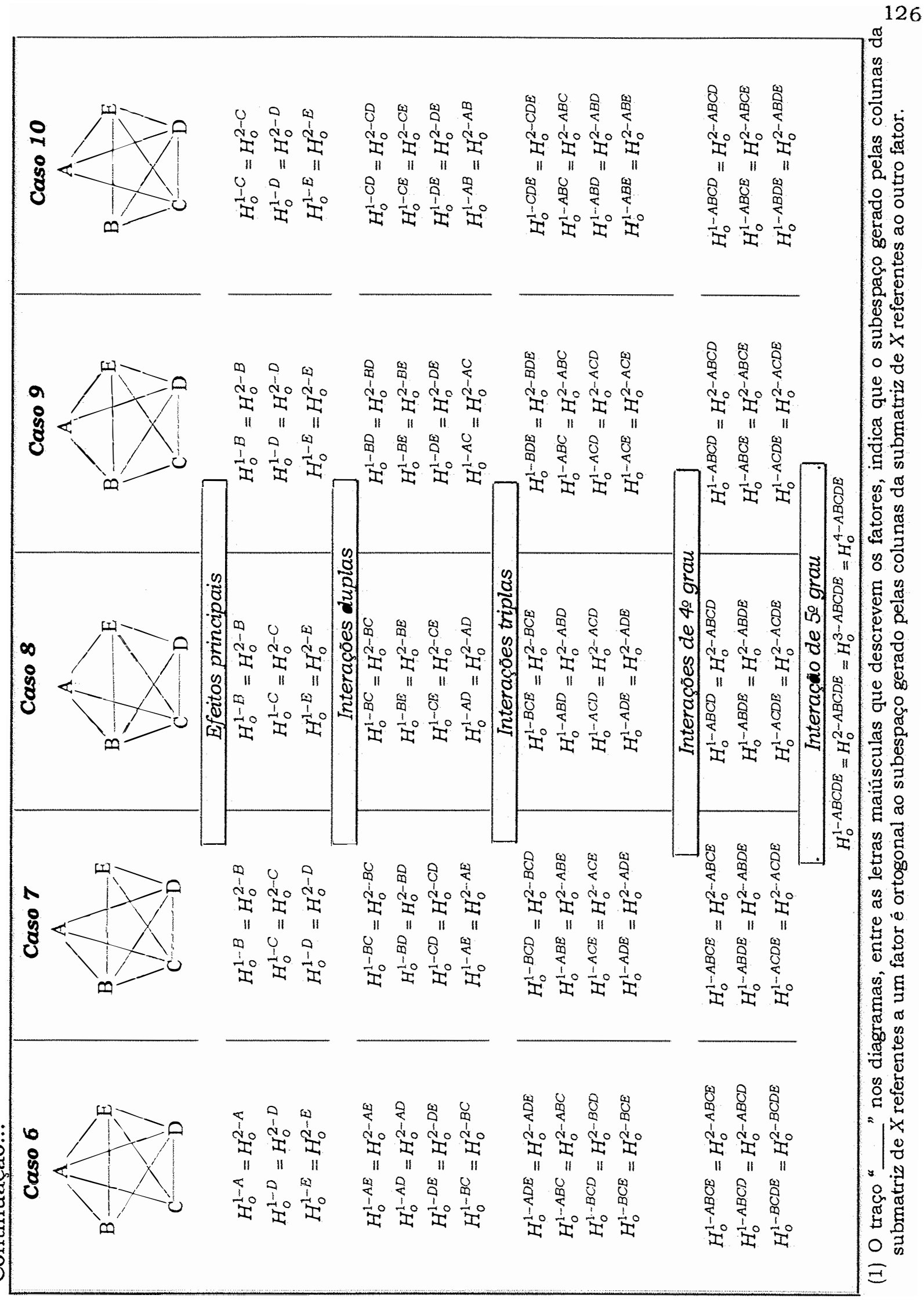




\section{CONCLUSÕES}

Neste estudo, buscou-se discutir e definir conceitos clássicos da estatística experimental, como ortogonalidade e análise de variância, visando formalizar a estrutura das funções estimáveis e das hipóteses testáveis nos modelos com e sem interações, utilizados para analisar delineamentos ortogonais e parcialmente ortogonais.

Face aos resultados obtidos, concluiu-se que:

\subsection{Quanto aos delineamentos ortogonais}

Um delineamento com $K$ fatores é dito ortogonal, se o subespaço gerado pelas colunas da submatriz de $X$ referentes a cada fator, após o ajuste de $\mu$, é individualmente ortogonal aos subespaços gerados pelas colunas das submatrizes de $X$ referentes aos outros $K-1$ fatores, após o ajuste de $\mu$.

\section{a) Modelos sem interações}

a.1) As funções paramétricas envolvendo um único fator são estimáveis. Então, as hipóteses sobre os efeitos de um fator, não contêm parâmetros que descrevem efeitos de nenhum outro fator, quer o conjunto seja ou não balanceado;

a.2) Dado que o modelo não contempla interações e que os conjuntos de dados não apresentam caselas vazias, condição necessária para ortogonalidade, então as hipóteses do tipo II são iguais às dos tipos III e IV. Nesse contexto, tanto para conjuntos balanceados quanto para conjuntos 
desbalanceados, um único quadro de análise de variância pode ser utilizado, simplificando sobremaneira a interpretação dos resultados;

\section{b) Modelos com interações}

b.1) As funções estimáveis de um fator contêm os parâmetros relativos ao fator e os respectivos componentes das interações nas quais o fator está presente;

b.2) As funções estimáveis para uma interação contêm parâmetros relativos a própria interação e parâmetros das interações de grau maior que o grau da interação em estudo;

b.3) Como o modelo contempla interações, as hipóteses do tipo II são diferentes das hipóteses dos tipos III e IV. Dessa forma, deve-se solicitar ao sistema de análise as hipóteses do tipo III, além da hipótese do tipo I encontrada no padrão dos programas.

c) Como conseqüência de a.1 e b.1 as hipóteses do tipo I ou hipóteses sobre médias ponderadas, são iguais às hipóteses do tipo II ou hipóteses sobre médias ponderadas ajustadas para todos os efeitos principais e interações;

d) A ortogonalidade, seja nos conjuntos balanceados ou nos desbalanceados, tem como conseqüência a igualdade entre todas as decomposições das somas de quadrados ajustadas de um mesmo fator ou interação. 


\subsection{Quanto aos delineamentos parcialmente ortogonais}

Um delineamento com $K$ fatores é dito parcialmente ortogonal, se os subespaços gerados pelas colunas das submatrizes de $X$ referentes, no máximo, a $K-2$ fatores, após o ajuste de $\mu$, são individualmente ortogonais aos subespaços gerados pelas colunas das submatrizes de $X$ referentes aos outros $K-1$ fatores, após o ajuste de $\mu$.

\section{a) Modelos sem interações}

As funções estimáveis do fator ou dos fatores nos quais os subespaços gerados são individualmente ortogonais aos subespaços gerados pelos demais fatores, contêm apenas os parâmetros relativos ao próprio fator, gerando hipóteses sob médias ponderadas iguais às hipóteses sob médias ponderadas ajustadas;

\section{b) Modelos com interações}

\section{b. 1) Efeitos principais}

As funções estimáveis do fator ou dos fatores, nos quais os subespaços gerados são individualmente ortogonais aos subespaços gerados pelos demais fatores, após o ajuste de $\mu$, contêm os parâmetros relativos ao próprio fator e os respectivos parâmetros das interações nas quais o fator está presente. Como conseqüência, as hipóteses sob médias ponderadas são iguaịs às hipóteses sob médias ponderadas ajustadas;

\section{b.2) Interações}

Funções estimáveis de interações que contêm os parâmetros relativos a própria interação e os parâmetros relativos à todas interações de 
grau maior à interação em estudo e apresentam hipóteses sob médias ponderadas iguais às hipóteses sob médias ponderadas ajustadas foram;

b.2.1) A interação que envolveu conjuntamente os fatores cujos subespaços gerados não foram ortogonais entre si;

b.2.2) As interações formadas pelos fatores cujos subespaços gerados foram individualmente ortogonais aos subespaços gerados pelos demais fatores;

b.2.3) As interações formadas pelos casos b.2.1 e b.2.2;

c) A ortogonalidade parcial não implica na desigualdade entre as decomposições das somas de quadrados para os efeitos principais e interações que não apresentaram hipóteses do tipo I iguais às hipóteses do tipo II;

\subsection{Considerações gerais}

O planejamento de delineamentos parcialmente ortogonais envolve a decisão antecipada do pesquisador sobre as hipóteses de seu maior interesse, pois nem todas as hipóteses sobre os efeitos principais e interações são testáveis.

Como conjunto completo é condição necessária tanto para os delineamentos ortogonais quanto para os parcialmente ortogonais sempre ocorre a igualdade entre as hipóteses dos tipos III e IV, bastando que apenas uma seja solicitada. 


\section{REFERENCIAS BIBLIOGRÁFICAS}

ADDELMAN, S. Orthogonal main-effect plans for assymetrical fatorial experiments, Technometrics, Alexandria, v.4, p. 21-46, 1962.

AZAIIS, J.M. Analyse de variance non-orthogonale: l'éxemple de SAS/GLM. Rev. Statist. Appl. Paris, v.17, n.2, p. 27-41, 1994.

CAMARINHA FILHO, J.A. Testes de hipóteses em modelos lineares com dados desbalanceados e caselas vazias. Piracicaba, 1995. 142p. Dissertação (M.S.)-Escola Superior de Agricultura "Luiz de Queiroz", Universidade de São Paulo.

CHRISTENSEN, R.A. Plane answer to complex questions. The teory of linear models. New York :Spring-Verlag, 1987. 380p.

CLAUSTRIAUX, J.J.; IEMMA, A.F. A propos des qalificatif complet, orthogonal et équilibré en analyse de la variance. Notes de Statist. et d'Inform. Gembloux, v.2, p.1-16, 1999.

FINNEY, D.J. Some orthogonal properties of the $4 \times 4$ and $6 \times 6$ latin squares. Ann. Eugenics, London, v.12, p.213-219, 1945a.

FINNEY, D.J. The fractional replication of factorial experiments Ann. Eugenics, London, v.12, p.291-301, 1945b. 
FINNEY, D.J. Orthogonal properties of the $5 \times 5$ latin squares. Ann. Eugenics, London, v.13, p.1-3, 1946a.

FINNEY, D.J. Orthogonal properties of the $6 \times 6$ latin squares. Ann. Eugenics, London, v.13, p.184-196, 1946 b.

HOCKING, R.R. The analysis of linear models. Monterrey: Brooks/Cole, $1985,385 p$.

IEMMA, A.F. Modelos lineares: uma introdução para profissionais da pesquisa agropecuária. Imprensa Oficial Est. Paraná, Londrina, 1987. $236 \mathrm{p}$.

IEMMA, A.F. Matrizes para a estatistica. Departamento de Matemática e Estatistica. ESALQ/USP, Piracicaba, 1989, 418p.

IEMMA, A.F. Álgebra de matrizes com aplicações em estatistica. Departamento de Matemática e Estatística. ESALQ/USP, Piracicaba, 1990, $411 \mathrm{p}$.

IEMMA, A.F. Testes de hipóteses em modelos com amostras desequilibradas. Gembloux, 1991. 101p.

IEMMA, A.F. Que hipóteses estatísticas testamos através do SAS em presença de caselas vazias? Scientia Agricola, Piracicaba, v.52, n.2, p. 210-220, 1995.

IEMMA, A.F.; CLAUSTRIAUX, J.J. Une condition necéssaire et suffisante pour que l'analyse des resultats d'une expérience croisée soit orthogonale. Faculté des Sciences Agronomiques. Gembloux, 1997. 21p. (seminário) 
IEMMA, A.F.; CLAUSTRIAUX, J.J. Sobre os termos “completo" “ortogonal e balanceado em análise de variância, Piracicaba, 1998, 36p. (Seminário).

IEMMA, A. F.; CLAUSTRIAUX, J. J. Étude des hypotèses de la variance à deux critèrés de classification: approache par léxemple. Notes de Statist. et d'Inform. Gembloux, v.3, p. 1-37, 1999.

IEMMA, A.F.; PALM, R. Les inverses généralisées et leur application dans le modéle lineaire. Gembloux. Notes de statist. et d'Inform., v.1, 25p, 1992.

IEMMA, A.F.; PALM, R.; CLAUSTRIAUX, J.J. Sobre a construção de projetores ortogonais. Revista de Matemática e Estatistica, São Paulo, v.11, p.133-142, 1993 .

IEMMA, A. F.; SANTANA, D. G. Delineamentos ortogonais com très fatores: teoria e hipóteses sobre os parâmetros. In: 45a REUNIĀO ANUAL DA REGIĀO BRASILEIRA DA SOCIEDADE INTERNACIONAL DE BIOMETRIA. Resumos. São Carlos, 2000. 186p.

JONES, R.M. On a property of incomplete blocks. Journal of Royal Statist. Soc B, London, v.21, p.172-179, 1959.

MONDARDO, M. Estimabilidade de funções estimáveis pasramétricas com dados desbalanceados através do PROC GLM dos SAS: Aplicações à pesquisa agropecuária. Piracicaba, 1994. 166p. Tese (Doutorado)-Escola Superior de Agricultura “ Luiz de Queiroz”, Universidade de São Paulo.

PEARCE, S.C. Field experimentation with fruit trees and othres perenial plants. Tech. Commun, Harpenden, v.23, 1953. 
PEARCE, S.C. The use and classification of non-orthogonal designs. Journal of Royal Statist. Soc, London, A. v.126, p.353-377, 1963.

PEARCE, S.C. The efficiency of block designs in general. Biometrika, Cambridge, v.57, n.2, p.339-346, 1970.

PEARCE, S.C. The agricultural field experiments. London: John Wiley \& Sons, 1983. 355p.

PREECE, D.A. Orthogonality and designs: a terminological muddle. Utilitas Mathematica, Winnipeg, v.12, p.201-223, 1977.

RAO, C.R. Linear statistical inference and its applications. New York: John Wiley \& Sons, 1945. 522p.

SANTANA, D. G.; IEMMA, A. F. Delineamentos ortogonais e parcialmente ortogonais: planejando para testar hipóteses de interesse. In: 45a REUNIĀO ANUAL DA REGIĀO BRASILEIRA DA SOCIEDADE INTERNACIONAL DE BIOMETRIA. Resumos. São Carlos, 2000. 186p.

SANTOS, E. S. Utilização de "softwares" estatísticos na interpretação de hipóteses com dados desbalanceados. Piracicaba, 1994. 230p. Tese (Doutorado)-Escola Superior de Agricultura "Luiz de Queiroz", Universidade de São Paulo.

SEARLE, S.R. Linear models data. New York: Wiley, 1971, 532p.

SEARLE, S.R. Arbitrary in linear models with unbalanced data. Commun. Statist. London v.2, p:181-200, 1980.

SEARLE, S.R. Linear models for unbalanced data. New York: Wiley, 1987, $536 \mathrm{p}$. 
SPEED, F.M.; HOCKING, R.R. ; HACKNEY, O.P. Methods of analysis of linear models with unbalanced data. Washington, Journal Amer. Statist. Assoc., Boston, v.73, p. 105-112, 1978.

TOCHER, K.D. The design and analysis of block experiments. Journal of Royal Statist. Soc. B, London, v.14, p.45-100, 1952.

YATES, F. Complex experiments. Jornal Royal Statist., London, v.2, p.181247, 1935.

$X$ [1990] SAS User's guide: statistics version 6 edition. Cary, SAS Institute, $846 p$.

X [1996] Minitab reference manual. Enterprise drive, State college. 